This document is downloaded from DR-NTU (https://dr.ntu.edu.sg) Nanyang Technological University, Singapore.

\title{
Improvement on geometry and hemodynamics of coronary artery bypass grafting : numerical and experimental studies
}

Xiong, Fangli

2007

https://hdl.handle.net/10356/5424

https://doi.org/10.32657/10356/5424

Nanyang Technological University

Downloaded on 26 Apr 2023 20:02:45 SGT 

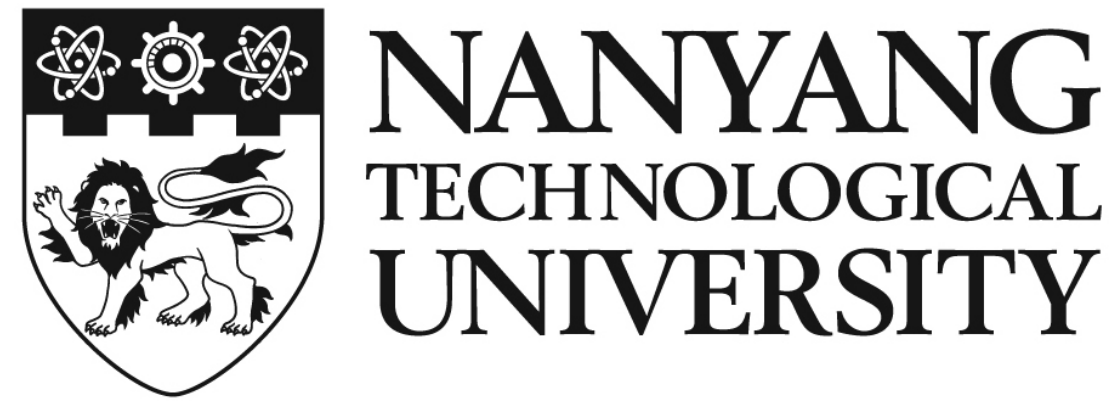

IMPROVEMENT ON GEOMETRY AND HEMODYNAMICS OF CORONARY ARTERY BYPASS GRAFTING: NUMERICAL AND EXPERIMENTAL STUDIES

\author{
XIONG FANGLI \\ SCHOOL OF MECHANICAL AND AEROSPACE ENGINEERING \\ NANYANG TECHNOLOGICAL UNIVERSITY




\title{
Improvement on Geometry and Hemodynamics of Coronary Artery Bypass Grafting: Numerical and Experimental Studies
}

\section{Xiong Fangli}

School of Mechanical and Aerospace Engineering

\author{
A thesis submitted to the \\ Nanyang Technological University \\ in fulfillment of the requirements for the degree of \\ Doctor of Philosophy
}




\section{ABSTRACT}

Impaired local hemodynamics associated with unfavorable anastomotic geometry has been implicated in the initiation and progression of intimal hyperplasia and atherosclerosis, the two vascular diseases for failing bypass anastomoses. Focused on the distal coronary anastomosis, this thesis seeks to determine critical hemodynamic wall parameters that indicate disease-susceptible sites as well as to optimize anastomotic geometry and hemodynamics pertaining to long-term graft viability.

Widely-hypothesized hemodynamic wall parameters, i.e. wall shear stress (WSS), oscillatory shear index (OSI), spatial and temporal WSS gradients (SWSSG and TWSSG), were calculated using a model of typical human coronary end-to-side anastomosis in physiological flow conditions. The results were correlated with histological data of disease distributions obtained through postmortem examination. The most causative wall parameters were determined as primary indicators for disease prediction and assessment of bypass hemodynamic performance. Subsequently, computational optimization of anastomotic geometry was conducted in terms of graftartery diameter ratio and angle, shape of anastomosis, graft-artery transitional curvature, and floor indentation. Concurrent in vitro experimentation using Particle Image Velocimetry (PIV) was coupled to measure flows in two life-size silastic models. Instantaneous velocity fields were recorded and WSS variations were calculated and compared with the corresponding numerical results.

Comparisons between the numerical study and PIV measurement in instantaneous WSS show satisfactory quantitative agreement with the largest discrepancy below $10 \%$ when 
estimated using an averaged normalized error. The level of the current validation is deemed adequate to provide a meaningful representation of unsteady coronary anastomotic flows. The study of hemodynamic factors implicates that the disease on the hood and graft body of the coronary anastomosis is likely caused by low WSS or high OSI, whilst low WSS, high OSI and high SWSSG combined are responsible for disease development on the artery floor. Using these factors as indicators of impaired hemodynamics, the study of geometric optimization demonstrates that the graft-artery diameter ratio is the most crucial factor that should be carefully controlled in the coronary revascularization surgery; the effect of the graft-artery angle is secondary. It is advisable to design a coronary anastomosis with a diameter ratio of about 1.5 , an angle of $30 \sim 45^{\circ}$, and as smooth a toe transition as possible to enhance hemodynamics and mitigate restenosis. Furthermore, a premanufactured biocompatible "sleeve" connecting the graft and recipient artery is proposed to use for reproducing and maintaining the designed geometry in vivo. 


\section{ACKNOWLEDGEMENTS}

First and foremost, I gratefully acknowledge my project supervisor, Dr. Chong Chuh Khiun. I have received invaluable assistance and suggestions from him. He has spent enormous amount of time and effort to enhance my ability in research and writing. I wish there is a better word than thanks to express my appreciation.

I would like to thank Prof. Dhanjoo N Ghista, Dr. Chua Leok Poh, and Dr. Tan Yong Seng in National Heart Center, for all the assistance that they had rendered to make the project possible.

The appreciation also goes to the technician Mr Yap Pow Khim and my colleagues in Thermal and Fluids Laboratory for the generous help and collaboration they continuously rendered during the course of my research.

Finally, my deep gratitude goes to my parents, who have given so much love to my life, and who were always there when times were difficult. Without them, none of these would be possible. 


\section{TABLE OF CONTENTS}

ABSTRACT

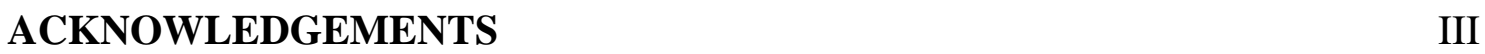

TABLE OF CONTENTS IV IV

LIST OF FIGURES VIII

$\begin{array}{ll}\text { LIST OF TABLES XV } & \text { XV }\end{array}$

$\begin{array}{ll}\text { LIST OF SYMBOLS XVI } & \text { XY }\end{array}$

1 INTRODUCTION AND OVERVIEW 1

1.1 ISCHEMIC HEART DISEASE AND TREATMENT 1

1.2 MOTIVATIONS 2

1.3 OBJECTIVES 5

2 BACKGROUND $\quad 8$

2.1 BACKGROUND ON CABG 8

2.1.1 The Coronary Circulation 8

2.1.2 Coronary Artery Bypass Graft Conduits 10

2.1.3 Recent Developments in CABG Technique 13

2.2 VASCULAR DISEASES AND HEMODYNAMICS 14

2.2.1 Molecular Mechanisms of Vascular Diseases 15

$\begin{array}{ll}\text { 2.2.1.1 Atherogenesis } & 17\end{array}$

2.2.1.2 Intimal Hyperplasia 19

2.2.1.3 Thrombogenesis 21

2.2.2 Hemodynamic Factors and Correlations to Localization of Lesions 24

2.2.3 Biological Responses of Blood Vessel Wall to Hemodynamic 28 Forces

2.2.4 Shear-dependent Transport of Blood Species 31 
2.3 CURRENT EFFORTS ON GEOMETRIC OPTIMIZATION OF DISTAL ANASTOMOSES

3 NUMERICAL METHOD AND THEORY

$\begin{array}{lll}3.1 & \text { INTRODUCTION } & 39\end{array}$

3.2 MESH GENERATION AND DEMONSTRATION OF MESH 40 INDEPENDENCE

3.3 NUMERICAL METHOD 47

3.3.1 Governing Equations and Flow Conditions 47

3.3.2 Solution Procedure 48

3.3.3 Data Analysis $\quad 52$

3.4 COMPARISON WITH LITERATURES 54

4 TEST ON DISEASE TRIGGERING MECHANISMS IN CORONARY 58 ANASTOMOSIS

$\begin{array}{lll}4.1 & \text { INTRODUCTION } & 58\end{array}$

4.2 NUMERICAL MODEL AND FLOW CONDITIONS 60

4.3 RESULTS 62

$\begin{array}{lll}4.4 & \text { DISCUSSION } & 70\end{array}$

5 GEOMETRIC INVESTIGATIONS AND OPTIMAL DESIGN OF $\begin{array}{ll}\text { DISTAL CORONARY ANASTOMOSIS } & 76\end{array}$

$\begin{array}{lll}5.1 & \text { INTRODUCTION } & 76\end{array}$

$\begin{array}{lll}5.2 & \text { FLOW CONDITIONS } & 78\end{array}$

5.3 EFFECT OF GRAFT-ARTERY DIAMETER RATIO 80

5.3.1 Numerical Model 80

5.3.2 Results: LAD Waveform 81

5.3.3 Results: RCA Waveform 91

$\begin{array}{lll}\text { 5.3.4 Discussion } & 98\end{array}$

5.4 EFFECT OF GRAFT-ARTERY ANGLE 102

5.4.1 Numerical Model 102

5.4.2 Results: LAD Waveform 102

$\begin{array}{ll}\text { 5.4.3 Results: RCA Waveform } & 104\end{array}$

$\begin{array}{ll}\text { 5.4.4 Discussion } & 107\end{array}$

5.5 EFFECT OF MILLER CUFF GEOMETRY 109

$\begin{array}{lll}\text { 5.5.1 Numerical Model } & 109\end{array}$ 
$\begin{array}{lll}\text { 5.5.2 Results } & 110\end{array}$

$\begin{array}{ll}\text { 5.5.3 Discussion } & 118\end{array}$

5.6 OPTIMAL DESIGN OF ANASTOMOTIC GEOMETRY 123

5.6.1 Numerical Model 123

5.6.2 Results: Effect of Transitional Curvature 124

5.6.3 Results: Effect of Floor Indentation 126

$\begin{array}{lll}\text { 5.6.4 Discussion and Summary } & 130\end{array}$

6 HEMODYNAMIC ASSESSMENT OF INTERPOSITION SLEEVE 133

$\begin{array}{lll}6.1 & 133\end{array}$

6.2 EFFECT OF PROXIMAL-DISTAL OUTFLOW RATIO 133

6.2.1 Numerical Model 133

$\begin{array}{lll}6.2 .2 \text { Results } & 135\end{array}$

6.3 EFFECT OF GRAFT-ARTERY NONPLANARITY 141

$\begin{array}{ll}\text { 6.3.1 Numerical Model } & 141\end{array}$

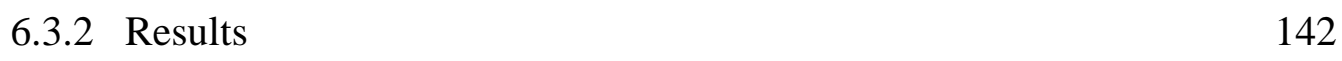

6.4 EFFECT OF SLEEVE WALL THICKNESS 147

6.4.1 Numerical Model 147

$\begin{array}{ll}\text { 6.4.2 Results } & 147\end{array}$

$\begin{array}{ll}6.5 \text { DISCUSSION } & 149\end{array}$

7 EXPERIMENTAL VALIDATION OF NUMERICAL RESULTS 155

$\begin{array}{lll}7.1 & \text { INTRODUCTION } & 155\end{array}$

$\begin{array}{lll}7.2 & \text { EXPERIMENTAL METHOD } & 157\end{array}$

$\begin{array}{ll}\text { 7.2.1 Experimental Model } & 157\end{array}$

7.2.2 Flow Circuit 160

7.2.3 Working Fluid and Tracer Particles 163

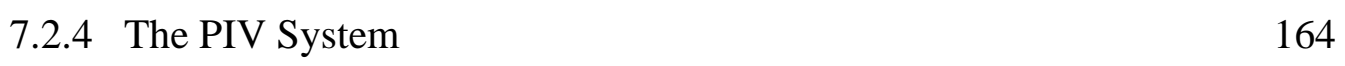

7.2.5 Parameters in PIV Measurement 165

7.2.6 Data Acquisition and Processing 168

7.2.7 Uncertainty and Error Analysis 170

$\begin{array}{lll}7.3 & \text { RESULTS: LAD MODEL } & 172\end{array}$

$\begin{array}{lll}7.4 & \text { RESULTS: RCA MODEL } & 179\end{array}$

$\begin{array}{lll}7.5 & \text { DISCUSSION } & 184\end{array}$ 
8 CONCLUSIONS AND RECOMMENDATIONS

$\begin{array}{llr}8.1 & \text { CONCLUSIONS } & 188\end{array}$

$\begin{array}{lll}8.2 & \text { RECOMMENDATIONS } & 191\end{array}$

$\begin{array}{ll}\text { REFERENCES } & 193\end{array}$

$\begin{array}{ll}\text { APPENDICES } & 210\end{array}$

A FLOW RESULTS FOR MILLER CUFF MODELS IN THE INFRA- 210 GENICULAR FLOW CONDITIONS

$\begin{array}{lll}\text { A.1 Flow Conditions } & 210\end{array}$

A.2 Results: 50:50 Flow Division Ratio 210

$\begin{array}{lll}\text { A.2.1 High Miller Cuff } & 211\end{array}$

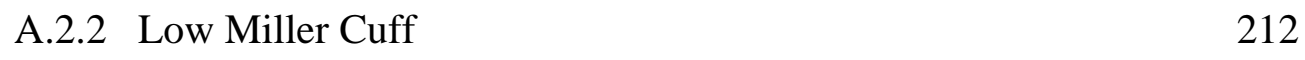

A.2.3 Long Miller Cuff 213

A.2.4 St Mary’s Boot 213

A.3 Results: 20:80 Flow Division Ratio 214

$\begin{array}{lll}\text { B EXPERIMENTAL APPARATUS } & 219\end{array}$

B.1 Gear Pump System 219

$\begin{array}{ll}\text { B.2 Illumination System } & 220\end{array}$

B.3 CCD Camera 222

$\begin{array}{lll}\text { B.4 FlowMap Processor } & 224\end{array}$ 


\section{LIST OF FIGURES}

1.1 Multi-vessel coronary bypasses: (a) complete arterial revascularization with right internal thoracic artery (ITA) to posterior descending artery (PDA); (b) composite ITA grafting with a saphenous vein graft (SVG) to PDA. The grafts are attached proximally to the ascending aorta and distally to the coronary artery downstream the stenosis.

1.2 Research Outline.

2.1 Cast of the coronary network of a human heart (left) and the main "skeleton" of the arterial network (right). In the left panel, the left coronary system is in light grey and the right coronary system is in black.

2.2 Cellular responses of the vessel wall to injury and inflammation.

2.3 Illustration of sagittal section of end-to-side anastomosis depicts sites of localization of intimal thickening at suture line, toe and heel, and artery floor.

2.4 Two methods of juxtaposing vein between PTFE graft and artery at the distal anastomosis: Miller cuff (top) and Taylor patch (bottom).

3.1 Mesh topologies (left) and WSS distributions (right) on the model surface for the two meshes with hexahedral and tetrahedral cells.

3.2 Direct comparison of cycle-averaged WSS on the artery floor between the two meshes with hexahedral and tetrahedral cells.

3.3 Topologies of the hexahedral meshes of increasing density.

3.4 WSS variation on the floor over both space and time for the meshes with (a) 15,448 cells, (b) 68,618 cells, (c) 108, 936 cells, and (d) 252, 592 cells.

3.5 Comparison of mean SWSSG on the floor between the four meshes.

3.6 Comparisons of the axial velocity component at (a) $0.25 d_{a}$, (b) $2 d_{a}$ and (c) $5 d_{a}$ distal to the toe between the numerical results from the present study (top) and the MRI results from Sherwin et al. (2000) (bottom). Also shown are velocity profiles extracted along the constant $\mathrm{z}$ centerline. Here, $d_{a}=8 \mathrm{~mm}$, is the host vessel diameter.

3.7 Numerically computed axial WSS along the bed against time using the LAD waveform from the present study (top) and the study of Ethier et al. (1998) (bottom).

4.1 The numerical model with relevant terminology. 
4.2 Waveforms simulating flows in arterial and venous grafts. Both were scaled to give a mean Reynolds number of 110 and a Womersley number of 2 based on the inlet vessel diameter of $5.3 \mathrm{~mm}$. Labels indicate time steps at which flow patterns are presented, including early negative flow (a), late negative flow (b), mid systole (c), diastolic acceleration (d), and diastolic deceleration (e).

4.3 Velocity vector distributions on the symmetry plane: (a) early negative flow, (b) late negative flow, (c) mid systole, (d) diastolic acceleration, and (e) diastolic deceleration. Streamtraces are used to enhance the data presentation.

4.4 Contour plots of cycle-averaged WSS: (a) LAD waveform, and (b) SV flow waveform.

4.5 Contour plots of OSI: (a) LAD waveform, and (b) SV flow waveform.

4.6 SWSSG variations along the artery floor over both space and time: (a) LAD waveform, and (b) SV flow waveform.

4.7 Variations of cycle-averaged SWSSG and OSI along the artery floor for the LAD waveform.

4.8 TWSSG variations along the artery floor over both space and time: (a) LAD waveform, and (b) SV flow waveform.

4.9 Variations of (a) SWSSG and (b) TWSSG along the hood and the upper wall of the distal artery over both space and time for the LAD waveform.

4.10 Statistical data of intimal thickening in the venous and arterial grafts.

5.1 The flow waveforms used in the study based on the work of Kajiya et al. (1986) and Matsuo et al. (1988). Labels indicate time steps at which flow patterns are presented. (a) LAD waveform: early negative flow $\left(\mathrm{t}_{1}\right)$, late negative flow $\left(\mathrm{t}_{2}\right)$, mid systole $\left(\mathrm{t}_{3}\right)$, diastolic acceleration $\left(\mathrm{t}_{4}\right)$, mid diastolic deceleration ( $\left.\mathrm{t}_{5}\right)$, and late diastolic deceleration $\left(\mathrm{t}_{6}\right)$; and (b) RCA waveform: mid systole $\left(\mathrm{t}_{1}\right)$, diastolic acceleration $\left(\mathrm{t}_{2}\right)$, and diastolic deceleration $\left(\mathrm{t}_{3}\right)$.

5.2 Geometric configuration of the model with relevant terminology.

5.3 Velocity vector plots in the symmetry plane during early negative flow for the models with (a) $\Phi=1: 1$, (b) $\Phi=1.5: 1$, and (c) $\Phi=2: 1$ in the LAD flow conditions. The black spots indicate positions of flow stagnation points.

5.4 Velocity vector plots in the symmetry plane during late negative flow for the models with (a) $\Phi=1: 1$, (b) $\Phi=1.5: 1$, and (c) $\Phi=2: 1$ in the LAD flow conditions.

5.5 Velocity vector plots in the symmetry plane during mid systole for the models with (a) $\Phi=1: 1$, (b) $\Phi=1.5: 1$, and (c) $\Phi=2: 1$ in the LAD flow conditions.

5.6 Velocity vector plots in the symmetry plane during diastolic acceleration for the models with (a) $\Phi=1: 1$, (b) $\Phi=1.5: 1$, and (c) $\Phi=2: 1$ in the LAD flow conditions. 
5.7 Velocity vector plots in the symmetry plane during mid deceleration for the models with (a) $\Phi=1: 1$, (b) $\Phi=1.5: 1$, and (c) $\Phi=2: 1$ in the LAD flow conditions.

5.8 Velocity vector plots in the symmetry plane during late deceleration for the models with (a) $\Phi=1: 1$, (b) $\Phi=1.5: 1$, and (c) $\Phi=2: 1$ in the LAD flow conditions.

5.9 Secondary flows during (a) mid systole, (b) diastolic acceleration, (c) peak diastole, and (d) diastolic deceleration at the cross-section of the host artery about $1 d_{a}$ downstream of the toe for the models with different diameter ratios.

5.10 Contours of cycle-averaged normalized WSS for the models with (a) $\Phi=1: 1$, (b) $\Phi=1.5: 1$, and (c) $\Phi=2: 1$ in the LAD flow conditions. The small panel shows the close-up view of the toe. The low shear area with normalized shear magnitude below 0.5 is labeled by " $L$ ".

5.11 OSI distributions for the models with (a) $\Phi=1: 1$, (b) $\Phi=1.5: 1$, and (c) $\Phi=2: 1$ under the LAD flow conditions. The small panel shows the closeup view of the toe.

5.12 Variations of SWSSG on the artery floor over both space and time in the LAD flow conditions for the models with (a) $\Phi=1: 1$, (b) $\Phi=1.5: 1$, and (c) $\Phi=2: 1$.

5.13 Variations of TWSSG on the artery floor over both space and time in the LAD flow conditions for the models with (a) $\Phi=1: 1$, (b) $\Phi=1.5: 1$, and (c) $\Phi=2: 1$.

5.14 Direct comparison of cycle-averaged SWSSG on the floor between the models with different diameter ratios.

5.15 Velocity vector plots in the symmetry plane during mid systole for the models with (a) $\Phi=1: 1$, (b) $\Phi=1.5: 1$, and (c) $\Phi=2: 1$ in the RCA flow conditions.

5.16 Velocity vector plots in the symmetry plane during diastolic acceleration for the models with (a) $\Phi=1: 1$, (b) $\Phi=1.5: 1$, and (c) $\Phi=2: 1$ in the RCA flow conditions.

5.17 Velocity vector plots in the symmetry plane during diastolic deceleration for the models with (a) $\Phi=1: 1$, (b) $\Phi=1.5: 1$, and (c) $\Phi=2: 1$ in the RCA flow conditions.

5.18 Contours of cycle-averaged normalized WSS for the models with (a) $\Phi=1: 1$, (b) $\Phi=1.5: 1$, and (c) $\Phi=2: 1$ in the RCA flow conditions. The small panel shows the close-up view of the toe. The low shear area with normalized shear magnitude below 0.5 is labeled by " $\mathrm{L}$ ".

5.19 OSI distributions for the models with (a) $\Phi=1: 1$, (b) $\Phi=1.5: 1$, and (c) $\Phi=2: 1$ under the RCA flow conditions. The small panel shows the closeup view of the toe.

5.20 Variations of SWSSG on the artery floor over both space and time in the RCA flow conditions for the models with (a) $\Phi=1: 1$, (b) $\Phi=1.5: 1$, and (c) $\Phi=2: 1$ 
5.21 Variations of TWSSG on the artery floor over both space and time in the RCA flow conditions for the models with (a) $\Phi=1: 1$, (b) $\Phi=1.5: 1$, and (c) $\Phi=2: 1$.

5.22 Geometric configuration of the model with relevant terminology.

5.23 Contours of cycle-averaged normalized WSS for the models with (a) $\alpha=$ $15^{\circ}$, (b) $30^{\circ}$, (c) $45^{\circ}$, and (d) $60^{\circ}$.

5.24 OSI distributions for the models with (a) $\alpha=15^{\circ}$, (b) $30^{\circ}$, (c) $45^{\circ}$, and (d) $60^{\circ}$.

5.25 Direct comparison of cycle-averaged SWSSG on the floor between the models with different angles.

5.26 Schematic picture of the model geometries: (a) Miller cuff, and (b) St. Mary's boot. The arrows indicate the main flow direction.

5.27 Velocity vector plots in the symmetry plane for the high cuff model in the LAD flow conditions: (a) early negative flow, (b) late negative flow, (c) mid systole, (d) diastolic acceleration, and (e) diastolic deceleration. The small panel shows the secondary flow in the A-A cross-section, about 0.5 $d_{a}$ distal to the cuff toe.

5.28 Velocity vector plots in the symmetry plane for the low cuff model in the LAD flow conditions: (a) early negative flow, (b) late negative flow, (c) mid systole, (d) diastolic acceleration, and (e) diastolic deceleration.

5.29 Velocity vector plots in the symmetry plane for the long cuff model in the LAD flow conditions: (a) early negative flow, (b) late negative flow, (c) mid systole, (d) diastolic acceleration, and (e) diastolic deceleration.

5.30 Velocity vector plots in the symmetry plane for the St Mary's boot model in the LAD flow conditions: (a) early negative flow, (b) late negative flow, (c) mid systole, (d) diastolic acceleration, and (e) diastolic deceleration.

5.31 Cycle-averaged WSS contours for the models with (a) high cuff, (b) low cuff, (c) long cuff, and (d) St Mary's boot.

5.32 OSI distributions for the models with (a) high cuff, (b) low cuff, (c) long cuff, and (d) St Mary's boot.

5.33 Schematic picture of the optimized geometries with (a) flat floor and (b) indented floor.

5.34 Velocity vector plots in the symmetry plane for the optimized (left) and conventional geometries (right): (a) early negative flow, (b) late negative flow, and (c) diastolic deceleration.

5.35 Comparison of cycle-averaged SWSSG for the models with smoothed graft-artery transitions. Here $\lambda$ is the aspect ratio at the toe.

5.36 Comparisons of cycle-averaged SWSSG between the conventional, high Miller cuff, St. Mary's boot and optimized geometries.

5.37 OSI distributions for the models with and without floor indentation. The small panel shows the close-up view of the floor. 
5.38 Comparison of cycle-averaged SWSSG between the models with and without floor indentation. Here $h$ is the height of the floor indentation.

6.1 Procedures for the conventional (left) and sleeve (right) techniques. In the conventional procedure, the graft is simply connected to the host artery; while in the sleeve procedure, a sleeve device is placed with one end inserted into the graft and the other two ends as well as the lower portion into the recipient artery.

6.2 Schematic picture of the sleeve model. The sleeve area is enclosed with the dashed lines.

6.3 Velocity vector plots for the sleeve model with zero proximal outflow: (a) early negative flow, (b) late negative flow, (c) mid systole, and (d) diastolic deceleration.

6.4 Velocity vector plots for the sleeve model with 40\% proximal outflow: (a) early negative flow, (b) late negative flow, (c) mid systole, and (d) diastolic deceleration.

6.5 Graph of neointimal thickness versus mean shear stress for PTFE tapered grafts in a canine model of intimal hyperplasia.

6.6 WSS distributions on the sleeve model for (a) the standard case ( $Q_{p}=$ $20 \%$ ), (b) $Q_{p}=0$, (c) $Q_{p}=10 \%$, (d) $Q_{p}=30 \%$, and (e) $Q_{p}=40 \%$.

6.7 OSI distributions on the sleeve model for (a) the standard case ( $Q_{p}=$ $20 \%$ ), (b) $Q_{p}=0$, (c) $Q_{p}=10 \%$, (d) $Q_{p}=30 \%$, and (e) $Q_{p}=40 \%$.

6.8 Comparison of cycle-average SWSSG between the cases with different proximal-distal outflow ratios.

6.9 Illustration of the sleeve model with nonplanar inflow geometry. Here $\beta$ is the nonplanar angle.

6.10 Axial velocity contour plots in the cross-sections extracted at (a) $\mathrm{X}=1.5$ $d_{a}$, (b) $3 d_{a}$, (c) $4.5 d_{a}$, and (d) $8 d_{a}$ for the planar (top) and $60^{\circ}$ nonplanar models (bottom) during the diastolic deceleration.

6.11 Secondary crossflows in the cross-sections extracted at (a) $\mathrm{X}=1.5 d_{a}$, (b) $3 d_{a}$, (c) $4.5 d_{a}$ and (d) $8 d_{a}$ for the planar (top) and $60^{\circ}$ nonplanar models (bottom) at the peak flow.

6.12 WSS distributions on the model surface for the models with (a) $\beta=0$, (b) $\beta=30^{\circ}$, and (c) $\beta=60^{\circ}$. For each model, two different views are displayed.

6.13 OSI distributions on the model surface for the models with (a) $\beta=0$, (b) $\beta=30^{\circ}$, and (c) $\beta=60^{\circ}$. For each model, two different views are displayed.

6.14 Illustration of the sleeve model with steps on the ends.

6.15 OSI distributions on the model surface for the models with step sizes of (a) $0.05 d_{a}$, (b) $0.08 d_{a}$, and (c) $0.1 d_{a}$. The small panel shows the 
close-up view at the sleeve-distal artery step.

7.1 Clear silicone rubber models representing anastomoses in the (a) LAD and (b) RCA regions.

7.2 Procedure of the physical model production.

7.3 System picture (top) and diagram (bottom) of the flow circuit.

7.4 Experimental and numerical inlet flow waveforms.

7.5 Optical system of planar pulsed particle image velocimetry. 166

7.6 Diffraction effects during particle image recording. 167

7.7 Pulse delay and pulse separation in double video frames.

7.8 Comparisons of transient flow fields between numerical simulation (left) and experimental measurement (right) for the LAD model: (a) $t=0.08$ cycle, (b) $\mathrm{t}=0.12$ cycle, (c) $\mathrm{t}=0.40$ cycle, $(\mathrm{d}) \mathrm{t}=0.48$ cycle, and $(\mathrm{e}) \mathrm{t}=$ 0.96 cycle.

7.9 Comparisons of WSS variations over the flow cycle at selected points of the anastomotic region between numerical simulation and experimental measurement for the LAD model.

7.10 Comparisons of WSS variations along the floor at selected time steps for the LAD model.

7.11 Comparisons of transient flow fields between numerical simulation (left) and experimental measurement (right) for the RCA model: (a) $t=0.24$ cycle, (b) $\mathrm{t}=0.60$ cycle, $(\mathrm{c}) \mathrm{t}=0.72$ cycle, $(\mathrm{d}) \mathrm{t}=0.88$ cycle, and (e) $\mathrm{t}=$ 0.96 cycle.

7.12 Comparisons of WSS variations over the flow cycle at selected points of the anastomotic region between numerical simulation and experimental measurement for the RCA model.

7.13 Comparisons of WSS variations along the floor at selected time steps for the RCA model.

A.1 Plot of the flow waveform used for the study. The flow rate is normalized with respect to the mean flow rate, $130 \mathrm{ml} / \mathrm{min}$. Labels indicate time steps at which flow patterns are presented.

A.2 Velocity vector plots in the symmetry plane for the high Miller cuff model during (a) acceleration, (b) peak flow, (c) mid-deceleration, (d) early systole, and (e) late diastole.

A.3 Velocity vector plots in the symmetry plane for the low Miller cuff model during (a) acceleration, (b) peak flow, (c) mid-deceleration, (d) early systole, and (e) late diastole.

A.4 Velocity vector plots in the symmetry plane for the long Miller cuff model during (a) acceleration, (b) peak flow, (c) mid-deceleration, (d) early systole, and (e) late diastole.

A.5 Velocity vector plots in the symmetry plane for the St Mary's boot model during (a) acceleration, (b) peak flow, (c) mid-deceleration, (d) early 
systole, and (e) late diastole.

A.6 Velocity vector plots at the mid-deceleration in the symmetry plane with a proximal-distal outflow division ratio of 20:80: (a) high cuff, (b) low cuff, (c) long cuff, and (d) St Mary's boot.

B.1 Pictures of (a) the gear pumps and (b) the calming chamber. 220

$\begin{array}{lll}\text { B.2 Picture of the illumination system. } & 221\end{array}$

$\begin{array}{ll}\text { B.3 Layout of the Gemini laser head. } & 222\end{array}$

B.4 Picture of the HiSense MkII CCD camera. 223

B.5 Architecture of the FlowMap PIV instrumentation. 224 


\section{LIST OF TABLES}

7.1 Summary of relative and normalized errors for the comparisons of the numerical and PIV WSS variations over the flow cycle at selected points.

7.2 Summary of relative and normalized errors for the comparisons of the numerical and PIV WSS variations along the floor at selected time steps.

7.3 Summary of relative and normalized errors for the comparisons of the numerical and PIV WSS variations over the flow cycle at selected points.

B.1 Gemini PIV Performance Specifications.

B.2 Specifications of the CCD camera. 


\section{LIST OF SYMBOLS}

$\alpha$

$\beta$

$d_{a}$

$d_{e}$

$d_{g}$

$d_{i}$

$d_{o}$

$d_{p}$

$\delta t$

$\delta z$

$\Delta t$

$\varepsilon_{\text {norm }}$

$\varepsilon_{r}$

$E_{\text {norm }}$

$E_{r}$

$f$

$\Phi$

$h$

$\lambda$

$\mu$

$v$

OSI

Q

$Q_{d} \quad$ Volume flow rate in the distal artery outlet segment

$Q_{g} \quad$ Volume flow rate in the graft

$Q_{p} \quad$ Volume flow rate in the proximal artery outlet segment

Graft-artery angle

Graft-artery nonplanar angle

Diameter of the host coronary artery

Image diameter

Diameter of the graft

Image distance in the PIV optical system

Object distance in the PIV optical system

Particle diameter

Pulse duration in the PIV illumination system

Depth of field

Pulse separation in the PIV illumination system

Normalized error

Percentage relative error

Average normalized error

Average relative error

Focal length of the camera lens

Graft-artery diameter ratio

Height of floor indentation

Aspect ratio of toe transitional curvature

Kinematic viscosity of the human blood

Dynamic viscosity of the human blood

Oscillatory shear index

Volume flow rate 


$\begin{array}{ll}\rho & \text { Density of the human blood } \\ \mathrm{Re} & \text { Reynolds number } \\ \mathrm{Re}_{\text {mean }} & \text { Mean Reynolds number } \\ \mathrm{S} & \text { Image-object scale factor } \\ \tau_{\text {norm }} & \text { Normalized wall shear stress } \\ \tau_{s} & \text { Poiseuille-type wall shear stress } \\ \tau_{S W S S G} & \text { Normalized spatial wall shear stress gradient } \\ \tau_{T W S S G} & \text { Normalized temporal wall shear stress gradient } \\ \tau_{w} & \text { Wall shear stress } \\ T & \text { Duration of a flow cycle } \\ u & \text { Blood flow rate } \\ u_{t} & \text { Transient flow rate } \\ W o & \text { Womersley number }\end{array}$




\section{CHAPTER 1}

\section{INTRODUCTION AND OVERVIEW}

\subsection{ISCHEMIC HEART DISEASE AND TREATMENT}

Oxygen-rich blood is delivered through the coronary arteries to the heart muscle or myocardium for its proper functioning. However, a reduction in the coronary blood supply to the myocardium as a result of ischemic heart disease causes ischemia and even myocardial cell death. This leads to angina pectoris and more severely, myocardial infarction (or "heart attack”). Ischemic heart disease is mainly caused by atherosclerosis, which refers to fatty lesions and cell proliferation inside the arterial wall. In the United States, this disease caused 1 out of 5 deaths in 2003 (Thom et al., 2006). In Singapore, ischemic heart disease as well as cancer and cerebrovascular disease have been the main causes of death since the 1970s, and accounted for $57 \%$ of all deaths in 1998 alone (Cutter et al., 2001).

Treatment for ischemic heart disease varies depending on the severity, number and position of atherosclerotic lesions as well as the clinical history of the patient. Drug therapy is used to reduce fat and cholesterol in blood or to trigger the smooth muscles of the arterial walls to relax, thus causing the arteries to dilate to increase blood flow. However, anginal symptoms cannot be effectively alleviated by medical treatment and restenosis occurs frequently (Parisi et al., 1992). Endarterectomy, the surgical removal of atherosclerotic plaque, is another option. This procedure is rarely adopted due to the poor results and the high risks involved in the operation (Brenowitz et al., 1988). Balloon angioplasty uses a balloon-tipped catheter to flatten the plaque, "restore" the 
arterial lumen and hence increase the blood flow. It treats the condition, but do not cure the cause. Recurrent narrowing has been reported in $17-25 \%$ of cases within 1 year (King et al., 1994) and 54\% within 5 years (Pocock et al., 1995). Another widely used technique is stenting, which usually follows angioplasty. It involves the deployment of a small metal device (scaffold) inside the artery to keep the artery open. This procedure also does not eliminate the cause and 1 out of 3 to 5 procedures suffer from short-term recurrences (Knight et al., 1997).

For life-threatening ischemic heart disease or intractable angina, coronary artery bypass grafting $(\mathrm{CABG})$ is frequently recommended, specifically for patients with lesions in the left main coronary artery or in multiple vessels, or with failure of nonsurgical management (e.g. medication and angioplasty) (Jones et al., 1996; Mark et al., 1994). In USA alone, over half a million Americans undergo CABG every year to relieve symptoms and prolong their lives. In this procedure, autogenous veins or arteries (called “grafts”), harvested from other areas of the body, are usually used to bypass blockages in the coronary arteries (Fig 1.1). Grafts made of synthetic material can also be an alternative when autogenous veins and arteries are not available. Triple and quadruple bypasses are often done during a single operation.

\subsection{MOTIVATIONS}

Although CABG has many benefits for the patient, failure as a result of restenosis has been the major concern, and remains unresolved. Angiographic studies have shown that $16 \%$ to $31 \%$ of vein grafts fail within 1 year (Bourassa et al., 1985; Goldman et al., 1989), and about half of all vein grafts are totally occluded within 10 years (Bourassa et al., 1984; FitzGibbon et al., 1991). Although arterial grafts show better performance in the mid- and longer-terms (Acar et al., 1998; Loop et al., 1986; Palatianos et al., 1993), 
their use are restricted by their shorter usable length and availability, especially for patients with multiple bypasses. In patients with failing grafts, repeated CABG is technically more challenging and associated with higher perioperative mortality as well as less symptomatic relief in comparison with the initial surgery (Cameron et al., 1979; De Feyter et al., 1985). On the other hand, the availability of suitable grafts can also be a limiting factor for repeated CABG.
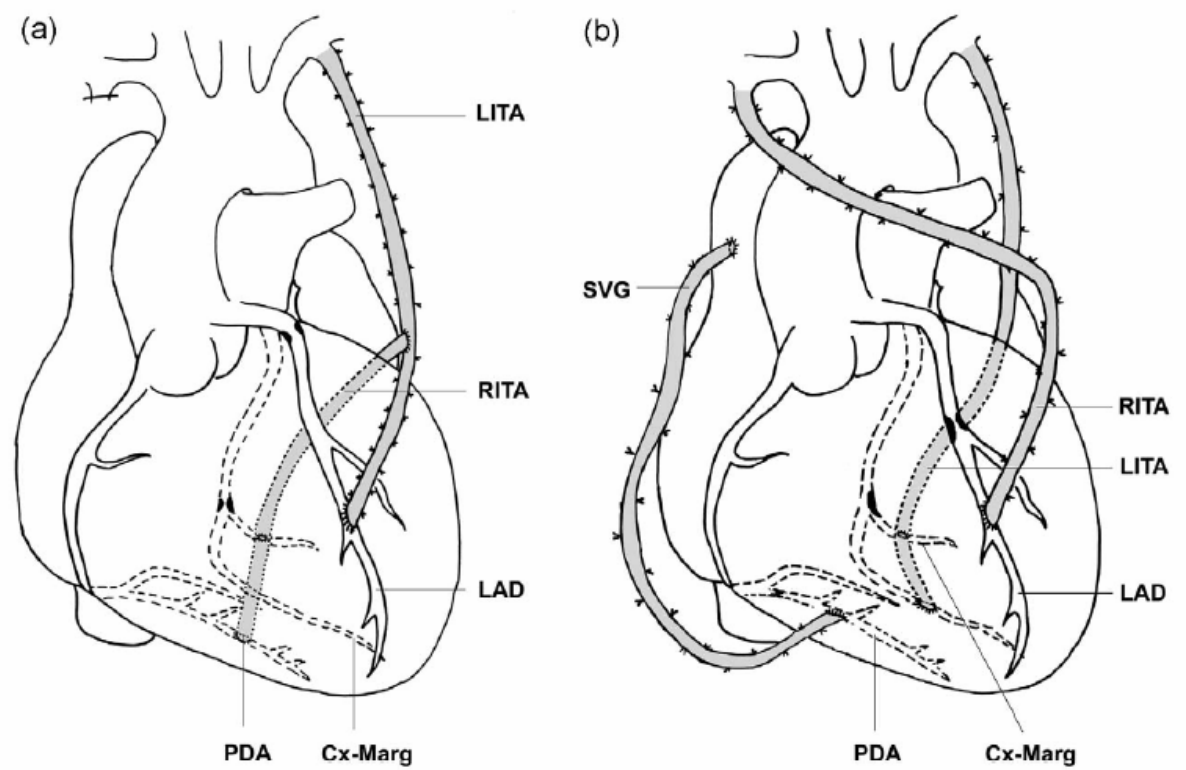

Fig. 1.1 Multi-vessel coronary bypasses: (a) complete arterial revascularization with right internal thoracic artery (ITA) to posterior descending artery (PDA); (b) composite

ITA grafting with a saphenous vein graft (SVG) to PDA. The grafts are attached proximally to the ascending aorta and distally to the coronary artery downstream the stenosis (from Paz et al., 2002, with permission).

Early failure (0-6 months) of coronary bypasses is primarily due to acute thrombosis caused by surgical trauma, and technical error as a result of high complexity in surgical manipulation (Fitzgibbon et al., 1996; Grondin et al., 1975). For long, cardiac surgeons have hoped for alternative ways to perform coronary bypasses less invasively with reduced surgical trauma, minimum operating time and standardized anastomotic quality. Several new devices for constructing the proximal graft-aortic anastomosis have been developed and applied in clinical practice (Hornik et al., 2003; Mack et al., 2003). 
Constructing the distal graft-coronary anastomosis, however, is technically more demanding and has needed further improvement for decades.

Late failure ( $>1$ year) of coronary bypasses is related to both fibromuscular intimal hyperplasia (IH) and recurrent atherosclerosis (Butany et al., 1998; FitzGibbon et al., 1996; Shelton et al., 1988). Both IH and atherosclerosis lead to a progressive intimal thickening at critical sites of an anastomosis, gradually occluding blood vessels. IH is triggered by the release of growth factors from the damaged endothelium or adherent platelets after the bypass surgery, followed by smooth muscle cell proliferation. Focal intimal thickening tends to develop at reproducible locations in distal end-to-side anastomoses in various vascular systems, such as the suture sites, hood, and host artery floor (or “bed”), where the mechanical environment is abnormal (Bassiouny et al., 1992; Lemson et al., 2000; Ojha, 2000). It has been suggested that while compliance mismatch and suture-line stress concentration are possible contributors to $\mathrm{IH}$ at the suture sites (Ballyk et al., 1998; Hofer et al., 1996; Weston et al., 1992), lesions on the hood and host artery floor are mainly the consequence of locally disturbed hemodynamics (Allaire and Clowes, 1997; Keynton et al., 2001). The precise mechanisms by which hemodynamics exerts its influences on the disease development remain unclear. Several hemodynamic factors have been postulated, including disturbed near-wall flow patterns, extremes in wall shear stress (WSS), high oscillatory shear index (OSI), as well as high spatial and temporal WSS gradients (SWSSG and TWSSG) (Ku et al., 1985; Lei et al., 1997; Nazemi et al., 1989; Ojha et al., 1994). It is well evidenced in peripheral artery studies that these hemodynamic parameters are profoundly altered by anastomotic geometry and hemodynamics can be effectively improved by geometric optimization (Fei et al., 1994; Hughes and How, 1995; Keynton et al., 1999; Lei et al., 1997; Moore et al., 1999). Thus far, however, such work on 
geometric optimization dedicated to the coronary region has either not been done, or has been limited to steady-state flow conditions which is not sufficient to represent physiological flows (Song et al., 2000).

Therefore, it is of great interest to optimize geometry and hemodynamics in the coronary anastomosis using more realistic pulsatile flow conditions to improve longterm graft viability, as well as to avoid acute graft failure by developing a better surgical technique for distal CABG.

\subsection{OBJECTIVES}

The overall goal of this research is to optimize geometry and hemodynamics in the distal coronary anastomoses for mitigating restenosis and enhancing graft viability. Figure 1.2 provides a summary of the research outline. In working towards this goal, the following objectives will be addressed:

1. To establish a relationship between hemodynamic factors and intimal thickening in distal coronary anastomoses. Optimizing anastomotic geometry relies firstly on understanding this relationship. The widely-hypothesized hemodynamic wall parameters, i.e. WSS, OSI, SWSSG and TWSSG, will be analyzed and compared in correlation to histological data of disease distributions obtained through postmortem examination of human coronary anastomoses in the literature. The most causative wall parameters dominating disease development will be determined and used as indicators for predicting disease susceptible sites and assessing bypass performance.

2. Geometric optimization for the distal coronary bypass anastomosis. Systematic investigations involving the effects of several important geometric factors (e.g. 
the graft-artery diameter ratio and angle, shape of anastomosis, toe transitional curvature, and floor indentation) on anastomotic hemodynamics will be conducted through numerical simulation. The results will provide a guideline to aid surgeons in making critical clinical decisions about CABG.

3. To assess hemodynamic performance for the optimized geometry based on a new sleeve technique. For reproducing the hemodynamically conducive geometry in vivo, the use of a premanufactured biocompatible "sleeve" connecting the graft and host artery is proposed. This new sleeve technique not only takes advantage of the optimized geometry to achieve improved hemodynamics, but it can also simplify and standardize surgical procedure to reduce surgical trauma and technical error. The hemodynamic performance of the sleeve will be assessed in terms of proximal-distal outflow ratio, graft-artery nonplanarity, and thickness of sleeve wall.

4. To verify the numerical results with in vitro bench-top measurements using the Particle Image Velocimetry (PIV) technique. Instantaneous velocity vector fields in life-size silastic anastomotic models will be measured, from which WSS distributions can be derived. The experimental data will be compared with the numerical results and discrepancies will be quantified with detailed error analyses. 


\section{Atherosclerosis in the coronary arteries}

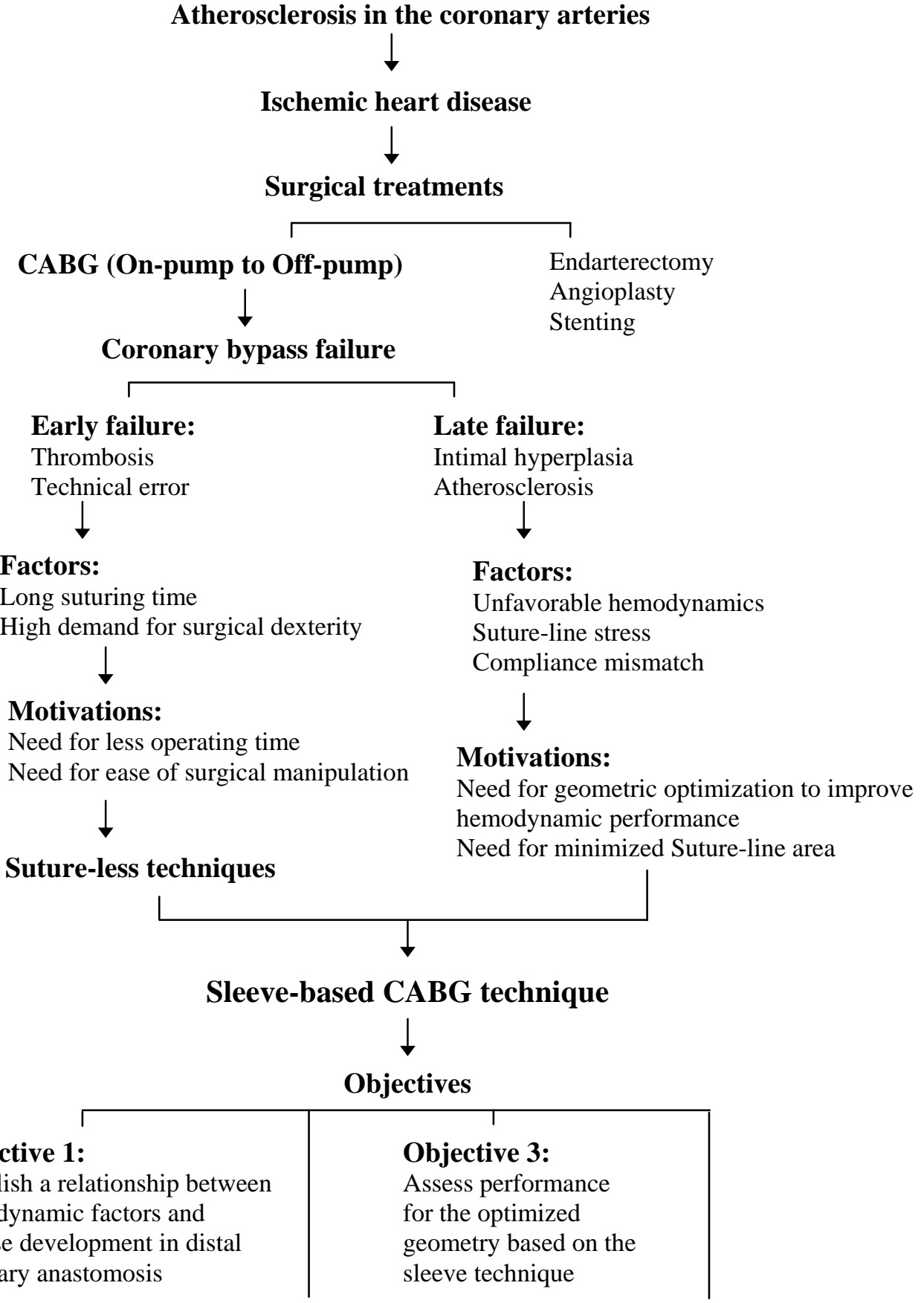

Objective 2:

Geometric optimization

Objective 4:

for distal coronary

anastomosis

Validate the numerical results by in vitro PIV measurements

Fig. 1.2 Research Outline. 


\section{CHAPTER 2}

\section{BACKGROUND}

\subsection{BACKGROUND ON CABG}

\subsubsection{The Coronary Circulation}

The coronary circulation provides oxygen-rich blood for the cardiac muscle to function and carries away oxygen-depleted blood. The coronary circulation consists of two main arteries: the left and right coronary arteries (LCA and RCA), which provide almost the entire blood supply of the heart. The left and right coronary arteries are the first branches of the ascending aorta and arise from the aortic sinuses immediately above its attachments to the heart. The left coronary artery, which divides into the left anterior descending artery (LAD) and the left circumflex artery (LCX), normally supplies blood to the left ventricle, left atrium and the interventricular septum, including the atrioventricular bundles. The right coronary artery, which divides into the right posterior descending artery (PD) and a large marginal branch, usually supplies blood to right ventricles, right atrium, and sinoatrial node (cluster of cells in the right atrial wall that regulates the heart's rhythmic rate). Smaller branches of the coronary arteries include: acute marginal (AM), obtuse marginal (OM), and diagonals. Figure 2.1 illustrates the cast of the coronary network. Detailed descriptions of the coronary anatomy can be found in Yacoub and Radley-Smith (1978).

The blood flow greatly varies in different vessels of the coronary system. Detailed information on flow characteristics in the main coronary vessels can be found in Sabiston (1974), Asakura and Karino (1990) and Ofili et al. (1993). Take, for instance, 
the two main vessels, the LAD and RCA. These two vessels are exposed to changing extravascular pressure during systole and diastole. During systole, large pressures are generated within the myocardial tissue. The myocardial vessels are compressed and tend to collapse, mainly in the left ventricle where the intraventricular pressure is highest. The extravascular compression causes the flow in the LAD to fall to zero and even become retrograde. With the onset of diastole, removal of the compression results in a large inflow into these vessels early in diastole. During the remainder of the cycle the flow in the LAD parallels the aortic pressure. The right ventricle, however, is a lowpressure chamber, so the RCA flow is not as influenced at systole as the LAD flow is. As a result, the LAD shows a predominantly diastolic flow and a portion of negative flow in systole, whereas the RCA shows about equal positive flows in systole and diastole.
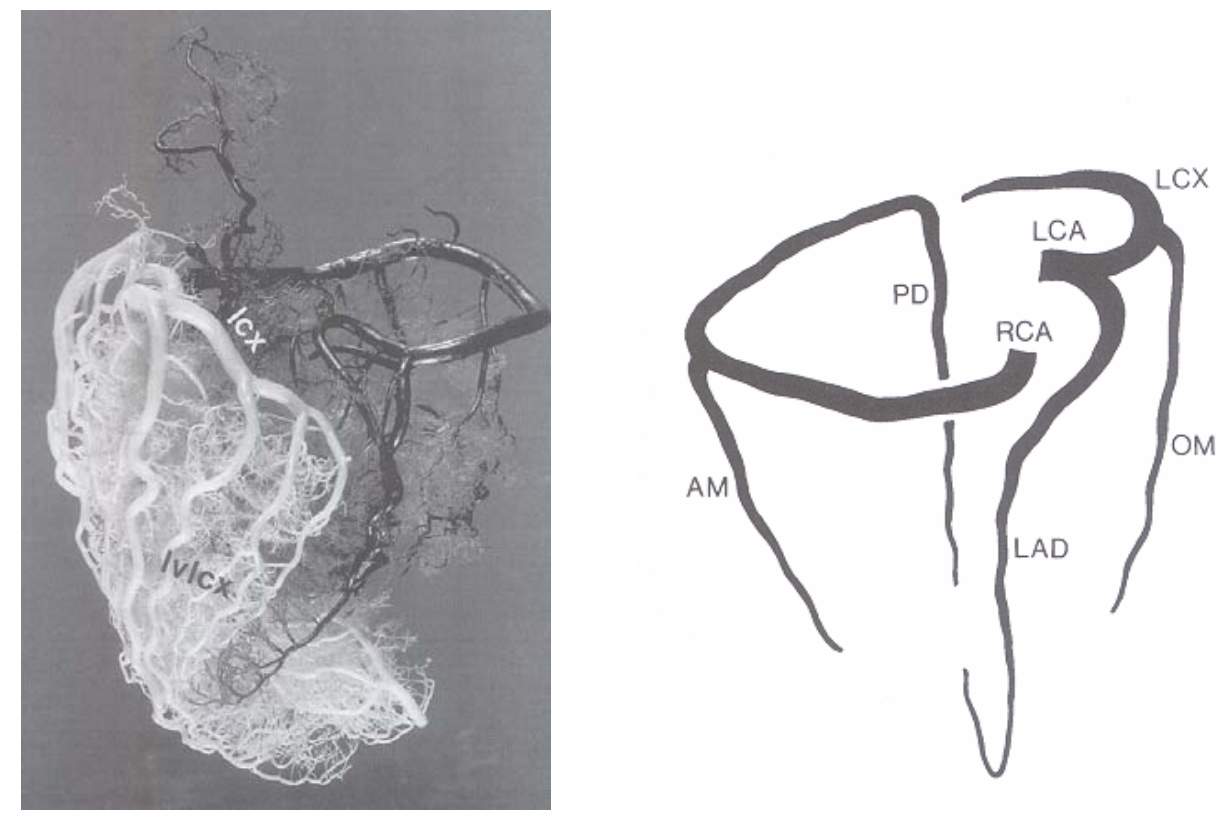

Figure 2.1 Cast of the coronary network of a human heart (left) and main skeleton of the arterial network (right). In the left panel, the left coronary system is in light grey and the right coronary system is in black (from Zamir, 2005, with permission).

In response to increases in demand caused by exercise or stress, the coronary circulation dilates by reducing vascular smooth muscle tone to increase blood flow. The circulation 
also autoregulates, altering vascular tone to maintain constant flow during changes in arterial pressure. Because of the ability to autoregulate, early stage coronary atherosclerosis rarely results in symptoms or damage to cardiac muscle. The flow resistance of normal epicardial arteries is very small. As the proximal artery becomes occluded, the peripheral circulation dilates. The ability of the circulation to augment flow in response to increased demand during exercise or other stress is diminished, and patients may exhibit chest pain during exertion. Not until the arterial lumen area is reduced by $80-90 \%$ does resting blood flow begin to diminish (Gould et al., 1990).

\subsubsection{Coronary Artery Bypass Graft Conduits}

The long saphenous vein (SV) is the preferred conduit for any bypass operations. It can usually give sufficient usable length of 65 to $75 \mathrm{~cm}$ and has a suitable internal diameter of 4 to $5 \mathrm{~mm}$ (Reardon et al., 1997). It also offers a better flow rate than other arteries like internal mammary artery (IMA) when acting as a conduit between the aorta and coronary arteries. Furthermore, the greater SV is less dependent on hemodynamic factors and less responsive to vasoconstriction related to inotropic agents (Privitera, 2001). Clinical studies have demonstrated that although the initial results were shown to be excellent, there is a slow but progressive degeneration in vein graft function. Vein graft disease can be divided into several stages: an early postoperative stage of thrombotic occlusion, an intermediate stage of intimal fibromuscular proliferation and a late stage of lipid deposition and atheromatous plaque formation which is usually associated with clot formation (Allen et al., 1995).

The IMA is a branch of the first part of subclavian artery with an average usable length of 20 to $25 \mathrm{~cm}$ and an average internal diameter of $2.5 \mathrm{~mm}$ but vary in size among individuals (Reardon et al., 1997). Recent studies have demonstrated the beneficial 
effects of IMA grafting on longer patency rates and have improved patient survival in myocardial revascularization (Eagle et al., 1999). Due to its high resistance to atherosclerotic disease, surgeons try to use one on every patient, if possible, but it is sometimes too short to reach some blockages on the back of the heart. Data from animal and human studies (Singh and Sosa, 1984; Pagni et al., 1996) have suggested that alterations in flow patterns, specifically competitive flow conditions, have been implicated in triggering the distal narrowing phenomenon in the IMA. This condition is devoid in vein grafts. Arterial grafts are known to retain their intrinsic properties in the endothelium and smooth muscle wall, allowing diameter adaptability to variable flow demands. The mechanisms involved in failure are not yet elucidated, but a flow-induced endothelial response may be responsible.

The internal thoracic artery (ITA) has proven excellent long-term patency and improved patient survival when used as a coronary bypass graft (Privitera, 2001). The left ITA is nearly always anastomosed to the LAD as a pedicled graft. It is a dynamic graft and can grow to accommodate the demands placed on it by the myocardium it supplies. The right ITA is not as commonly used but can be used as a free graft. The good long-term performance for ITA graft was only reported in the left coronary system (Dietl et al., 1995). However, reduced patency rate of in-situ right ITA has been reported when grafted to the distal right coronary system (Dion et al., 2000). A recent study of Paz et al. (2002) compared the results of complete arterial revascularization of the left and right coronary system with bilateral skeletonized ITAs with the results of bilateral ITA grafting and right coronary system revascularization using SV grafts. They found that lower return of angina in the SV group made SV grafting preferable for the right coronary system. 
Since the studies involving the ITA, there has been increased interest in other arterial conduits. These include the gastroepiploic artery (GA), the inferior epigastric artery (IEA), and the radial artery (RA), which have all been considered as supplements to the ITA (Barner, 1998). Although a theoretical advantage for the use of arterial conduits exists, their superiority has yet to be borne out in long-term studies. In addition, these arteries are more difficult to harvest, more easily damaged, more demanding to anastomose owing to their fragility and small size, and more compromised by spasm or technical error (Barner, 1998). Intimal hyperplasia is much less common in arterial grafts such as IMA, ITA and GA than in SV grafts, suggesting that different biological properties of the vessel wall between these arteries and this vein must be involved.

The conduits made of synthetic material such as Dacron and Expanded Teflon (ePTFE) are usually preferred for large peripheral artery reconstruction. It can also be an alternative for coronary artery grafts when there is lack of autologous vein or artery. The ePTFE is a synthetic polymer of carbon and fluorine produced by mechanical stretching (Rutherford, 1989). The main advantages of ePTFE include impermeability to blood, resistance to aneurismal formation, and ease of declotting. However, the cilinial results of the synthetic materials remains poor owing to their inherent thrombogenicity and neointimal hyperplasia (Rutherford, 1989). The seeding with endothelial cells and subsequent proliferation of these cells into a confluent monolayer along the graft are suggested to inhibit early failure such as platelet deposition leading to thrombus formation (Sterpetti et al., 1992). Several complementary approaches to enhance the results of endothelial cell seeding have been developed, including in vitro and in vivo conditioning of the cells and modifying cell phenotype through gene transfer. The purpose is to induce a suitable functional phenotype in the cells and to maintain it despite adverse signals from inflammatory stimuli, mechanical injury and 
inadequate extracellular matrix for cell differentiation on synthetic surfaces (Canfield and Schor, 1995). However, the results of endothelial cell seeding onto synthetic grafts are variable and the clinical utility remains to be defined.

\subsubsection{Recent Developments in CABG Technique}

In the conventional cardiopulmonary bypass $(\mathrm{CPB})$ surgery, the patient is connected to the heart-lung machine, which supplies oxygen to the blood and circulates blood to other parts of the body during the surgery while the heart muscle is stopped before the grafting is done. The entire surgery can take four to six hours. Considering that the use of the heart-lung machine may lead to some loss of memory and mental clarity, the offpump coronary artery bypass (OPCAB) has been introduced and becomes increasingly popular, which allows the bypass to be connected while the heart is still beating (Roche and Stengle, 1973). Recent results of OPCAB operations in low-risk and high-risk patients showed excellent results and improved neurocognitive outcomes compared with patients undergoing operations using CPB (Trehan et al., 2001). It is estimated that by the year 2005 more than $50 \%$ of surgical coronary revascularizations will be performed without CPB (Shennib, 2001). Another alternative is the use of smaller incisions that avoid splitting the breastbone and reduce recovery time (Falk et al., 2000), which is referred to as Minimally Invasive Direct Coronary Artery Bypass (MIDCAB). However, the number of cases eligible for this method is limited for the small minority with single vessel disease only (Falk et al., 2000).

The trend of using OPCAB and MIDCAB stimulates the development of anastomotic suture-less devices to make anastomosis simple and of consistent high quality, and to avoid the risks associated with aortic clamping. Suture-less anastomosis means performing vascular reconstruction without using hand-sewing or hand-tying knots 
(Tozzi et al., 2002). The devices that are now available for clinical use include Q-CAB and CorLink for proximal anastomosis, and GraftConnector and SIM Distal Connector for distal anastomosis. They generally provide less graft manipulation, avoid aortic cross-clamp, and reduce operating time. However, all the aortic connectors construct an anastomosis with a $90^{\circ}$ angle which is predisposed to graft kinking and may compromise peri-anastomotic hemodynamics (Tozzi et al., 2002). The surgical manipulation is more complicated for the distal anastomosis due to the smaller vessel size. The GraftConnector device allows the surgery to be completed in less than 3 minutes on beating hearts without stabilizers. However, this technique also provides little improvement on the anastomotic geometry that is essential for the long-term graft patency. In summary, all the existing devices for constructing both proximal and distal anastomoses place little emphasis on the improvement of anastomotic geometry and hemodynamics. It is imperative to develop a new technique which can not only lower the technical demand, minimize the operating time and standardize the quality of the surgical procedure to avoid the short-term failure, but also ensure hemodynamically conducive anastomotic geometry to achieve the long-term patency.

\subsection{VASCULAR DISEASES AND HEMODYNAMICS}

Atherosclerosis, intimal hyperplasia and thrombosis, are major contributors to restenoses in vascular bypass systems. Both atherosclerosis and intimal hyperplasia lead to a progressive intimal thickening at critical sites mainly caused by local smooth muscle cell proliferation inside the vessel wall, which would gradually occlude blood vessels. There are basic differences in pathogeneses of the two main causes of intimal thickening. The stimuli to atherosclerosis are systemic risk factors (e.g. hypertension, smoking, hyperlipidemia, diabetes mellitus, etc.), and intimal thickening involves macrophages, lipids, and smooth muscle cells, whereas intimal hyperplasia involves the 
release of growth factors from the endothelium or adherent platelets, followed by smooth muscle cell proliferation. Thrombosis, on the other hand, encompasses interactions between blood cells, principally platelets and the coagulation proteins, and may produce rapid occlusion. Local hemodynamic forces control function of the endothelium, endothelial gene expression and phenotype switching, smooth muscle cells proliferation, transport of macromolecules in blood (e.g. LDL), as well as monocyte (platelets, leukocytes) accumulation; therefore, it is not surprising that these forces have been implicated in the pathogeneses of the vascular disorders. The close correlation between hemodynamics and vascular diseases have stimulated much work to explore exactly what hemodynamic factors are best associated with the disease processes. There are generally two categories: i) the focus on the relationship between hemodynamic factors and the localization of lesions, including numerical simulations, in vitro bench-top experiments and in vivo measurements (in animals or through postmortem examinations); ii) the focus on the relationship between hemodynamic factors and vascular biology, mainly including in vitro cell culture experiments and in vivo animal studies. In the following, the mechanisms on atherosclegenesis, IH and thrombogenesis are described. Subsequently, the results from the studies in the two categories are reviewed and discussed.

\subsubsection{Molecular Mechanisms of Vascular Diseases}

The artery wall consists of three distinct layers as illustrated in Fig. 2.2A: the intima, which is lined by a monolayer of endothelial cells (called endothelium); the media, which includes almost entirely smooth muscle cells and extracellular matrix with some elastic lamellae; and the adventitia, which is composed of fibrous tissue containing elastic fibers and fibroblasts, fat cells, and interstitial matrix (Newby and Zaltsman, 2000). 
The endothelium, as the barrier of the blood vessel wall, provides an antithrombotic nonsticky surface for blood flow, synthesizes molecules that modulate vessel tone and diameter, and passes nutrients carried by the blood to the smooth muscle in the inner layer (Verma and Anderson, 2002). The endothelial cells are important in the balance of coagulation and fibrinolysis, regulate platelet function and vascular growth. In addition, endothelial cells play a pivotal role in regulation of vascular tone through the release of a variety of biological substances. Nitric oxide (NO), For example, is one of the important molecules involved in the regulation of blood vessel in response to altered flow (Verma and Anderson, 2002). One of the primary functions of NO involves the regulation of vascular caliber to modulate blood flow and normalize endothelial shear stress and cyclic strain. The molecular mechanisms of NO activity include roles in signaling, free radical scavenging and posttranslational protein modification. Secretion of NO into the vessel lumen causes reduction of the adhesive properties of platelets and leukocytes, and secretion of NO into the smooth muscle cells of the media causes vasorelaxation and inhibits smooth muscle cell proliferation. Attenuation of NO is one of the earliest biochemical changes preceding endothelial dysfunction. In addition to NO, growth factors together with extracellular matric cues regulate the growth of new blood vessels. New blood vessels develop by sprouting of existing vessels in response to injury. A variety of growth factors (and cytokines) coordinate the reprogramming of endothelial cells, mesenchymal cells, and monocytes associated with new vessel formation. For example, platelet-derived growth factor (PDGF) stimulates the proliferation of smooth cells and fibroblastsv (Ferns et al., 1991). Other common atherogenic components include basic fibroblast growth factor (bFGF), transforming growth factor $\beta$ (TGF- $\beta$ ), monocyte chemoattractant protein-1 (MCP-1), Metalloproteinases (MMPs), vascular cell adhesion molecule (VCAM-1), intercellular 
adhesion molecule (ICAM-1), E-selectin and P-selectin (Ross, 1995). The bFGF is released from damaged endothelial and arterial smooth muscle cells, and its infusion stimulates the proliferation of smooth muscle cells in the media (Lindner and Reidy, 1991). The activities of these atherogenic substances in stimulating lesion development are illustrated in Fig. 2.2B,C. Each stage of atherosclerosis and intimal hyperplasia is regulated by unique but overlapping molecular events dominated by growth factors, cytokines, and proteases, which are discussed in the following.

\subsubsection{Atherogenesis}

The process of atherogenesis is complicated and involves a lot of physiological and pathological events. Classified by different stages of the disease, atherosclerotic lesions involve three types: the fatty streak, the intermediate or fibrofatty lesions, and the ultimately advanced lesions or fibrous plaque (Ross, 1995). The process of atherosclerosis is summarized below. Detailed descriptions can be found in the studies of Ross (1993); Ross (1995); Munro and Cotran (1988); Steinberg (1987).

The initial event in the development of atherosclerosis is LDL deposition within the artery wall through incorporation into the extracellular matrix. High surface concentration of LDL can stimulate expression of VCAM-1, which mediates adhesion of monocytes to the endothelium. Successively, high concentration and long residence time of LDL in the intima cause itself to become oxidized. Modified LDL stimulates the endothelium to produce chemoattractants, e.g. MCP-1 and M-CSF, and to induce expressions of adhesion molecules, e.g. VCAM-1, ICAM-1, E-selectin and P-selectin. The expression of such substances promotes monocyte binding to the endothelium. Monocytes then enter the intima and migrate into macrophages. Interactions between the overlying endothelium and the monocyte-derived macrophages could lead to 
chemoattraction of monocytes and macrophage replication. Macrophages are the principle source of foam cells in the lesions since they take up lipid and oxidized LDL through scavenger receptor or bulk phase endocytosis. The formation of foam cells and their continued accumulation in the intima lead to the first ubiquitous lesion of atherosclerosis, the fatty streak. The fatty streak consists of an intimal collection of lipid-filled, monocyte-derived macrophages, with a varying number of $\mathrm{T}$ lymphocytes. The accumulation of lipid-filled macrophages, or foam cells, represents the bulk of the lesion and occupies several layers of the intima of the artery.

Further, the uptake of oxidized LDL and other substances within the lesions of atherosclerosis may serve as a stimulus to induce gene expression for several growthregulatory molecules and cytokines, which can be chemotactic agents, growth agonists, or antagonists. The chemotactic agents can induce monocyte proliferation, smooth muscle cell proliferation, and endothelial proliferation. Activated macrophages can also produce a series of chemotactic molecules for other monocytes, for endothelial cells, and for smooth muscle cells. Once smooth muscle cells are attracted into the lesion by chemotactic factors generated by activated macrophages, the interactions between macrophages and smooth muscle and between smooth muscle themselves could play critical roles in generating intermediate or fibrofatty atherosclerotic lesions. The fibrofatty lesion consists of layers of lipid-filled macrophages and $\mathrm{T}$ cells that alternate with layers of varying number of smooth muscle cells surrounded by a relatively poorly developed connective tissue matrix of fine collagen fibrils, elastic fibers, and proteoglycans.

As the lesions continue, the cells are rearranged, leading to the ultimately advanced lesions or fibrous plaque. The fibrous plaque is characterized by a dense cap of fibrous 
connective tissue that contains numerous smooth muscle cells surrounded by dense layers of connective tissue matrix, made up principles of collagen with some elastic fibers.

\subsubsection{Intimal Hyperplasia}

Typical IH is composed of about $20 \%$ smooth muscle cells, macrophages and lymphocytes (Kraiss and Clowes, 1997). The cellular and molecular basis of IH has been explored by many researchers (Clowes, 1993; Kraiss and Clowes, 1997; Jawien et al., 1992; McNeil et al., 1989). The most extensively characterized model is the rat carotid balloon injury (Jawien et al., 1992). Although IH after implantation of a vascular graft is different from the arterial balloon injury in terms of the initiation and the speed of the disease development, it is generally thought that the sequence of events in the development of $\mathrm{IH}$ is similar. There are four distinct stages or "waves" leading to neointima formation in normal arteries. This sequence of events begins within hours and concludes within 6 to 12 weeks at which point the neointima remains stable or regresses (Jawien et al., 1992).

The initial stage is medial smooth muscle cell replication or proliferation (24-72 hours) (Clowes et al., 1983). Endothelial cell damage induces thrombus formation, which successively stimulates vascular smooth muscle cell proliferation within 24 hours. Damaged endothelium, unlike intact endothelium, can fail to produce enough NO and heparan sulfate, both of which are inhibitors of smooth muscle cell proliferation (Kraiss and Clowes, 1997). Vascular smooth muscle cell proliferation can also be promoted by basic fibroblast growth factor (bFGF) and many possible other mediators which are released from dying or damaged EC and VSMC (Jawien et al., 1992; McNeil et al., 1989). The second stage is migration of medial smooth muscle cells from the media 
across the internal elastic lamina to form a new intimal layer or "neointima" (4-7 days) (Clowes et al., 1983). VSMC are distributed in an extracellular matrix which contains the basement membrane, the inner elastic lamina and the interstitial matrix (Murphy and Doherty, 1992). Injury induces degradation of the extracellular matrix and activation of matrix metalloproteases (MMPs). The matrix synthesis and matrix metalloproteases (MMPs) exert continual effects on the extracellular matrix, stimulating smooth muscle cell migration (Murphy and Doherty, 1992). Several molecules such as TGF- $\beta$ and bFGF, which increase after injury, all stimulators of migration (Lindner and Reidy, 1991). In vivo, one of the three isoforms of PDGF, PDGF-BB, is also found to play a role in migration (Jawien et al., 1992). In vitro, the PDGF-BB isoform results in both SMC migration and proliferation, whereas PDGF-AA has only a proliferative effect (Koyama et al., 1994). The third stage is a second wave of replication in smooth muscle cells within the neointima (1-4 weeks), which is not bFGF-dependent but driven by a separate set of growth factors (Clowes et al., 1983). Once VSMC arrive in the intima of the rat artery, neointimal cells closest to the lumen may replicate for weeks or months. The neointima can be stimulated to show a further increase of replication. The process of smooth muscle cell production of extracellular matrix to further expand neointimal mass can be called a "fourth wave" and involves at least TGF- $\beta$, bFGF and Ang II as agonists (Clowes et al., 1983). The process of VSMC replication and volume of extracellular matrix production appear to be tightly linked, with constant proportione of VSMC to extracellular matrix volumes (Kohler et al., 1991).

The morphological sequence of the development of IH in venous graft is supposed to be different from in native arteries. Vein grafting is followed by a rapid deposition of molecules such as platelets and leukocytes. These can secrete cytokines to stimulate VSMC proliferation and migration (Golledge, 1997). About two months after vein 
grafting, enhanced platelet activation (Gershlick et al., 1984) and an increased production of PDGF and BFGF (Sterpetti et al., 1996) are found. A study showed that ECs in the venous graft continue to proliferate at a high rate even though they are plenty in amount on the intima (Zwolak et al., 1989). The evidence also suggests that ECs in healed venous grafts do not prevent the development of occlusive intimal thickening like arterial endothelium (Berger et al., 1972). When the vein is handled carefully during operation, most of the venous ECs are retained on the intima after arterial flow is restored; however, venous ECs fail to serve as a competent blood flow transducer in arterial conditions. In addition, the vasodilatory response to acetylcholine including the NO pathway is reported to be lower in human saphenous veins than in internal mammary arteries (Luscher et al., 1988), which may explain why autologous vein grafts are more prone to intimal thickening than arterial grafts in coronary reconstruction surgery.

\subsubsection{Thrombogenesis}

Acute coronary thrombosis is an extremely significant health problem as it would suddenly occlude the blood vessel and lead to unstable angina pectoris and acute myocardial infarction. A thrombus is simply an accumulation or aggregate of platelets, held together by fibrous strands penetrating throughout the interior and exterior of the platelet clump (Colman, 1993). Two cell types, platelets and red blood cells, constitute a thrombus. The cells are held together by molecules in the cell membrane of the platelets, called membrane glycoproteins (GPs), by a variety of plasma proteins and by a network of polymerized plasma protein called fibrin (Kieffer and Phillips, 1990). Acute thrombosis, at a functional level, is described by three platelet functions, i.e. adhesion, activation and aggregation, and the coagulation cascade. Platelets adhere to proteins in the surface via platelet membrane GPs. Subendothelial tissue and atheroma 
contain collagen and von willebrand factor (vWF) which are binded to platelets via GPs. GPIb mediates a rapid but transient binding to vWF, whereas GPIIb/IIIa mediates more permanent binding. GPIIb/IIIa can bind to many other plasma and vessel wall proteins including fibrinogen, fibrin, fibronectin, thrombospondin, and vitronectin (Savage et al., 1996). Platelet activation means that platelet functions are triggered by chemical or physical stimuli. Abnormal flow or shear stress can activate platelets (Hellums, 1994). Implanted synthetic materials are also a source of platelet activation. The most important activation function is a conformational change in GPIIb/IIIa that allows it to bind to plasma proteins. Activation causes shape change with pseudopod extension to enhance adhesion and decrease the resistance of platelets to aggregation (Colman, 1993). Platelet aggregation is crucial to formation of a platelet plug. It is shown that activated platelets bind together via fibrinogen or fibrin and GPIIb/IIIa at lower shear rates, and via vWF at higher shear rates (Alevriadou et al., 1993; Markou et al., 1993). Convection patterns and high residence time in the regions with separated flow, flow stagnation or recirculation are shown to induce both platelet activation and aggregation (Bluestein et al., 1997; Bluestein et al., 1996). Exposure of a thrombogenic surface is also likely to trigger coagulation cascade and ultimately fibrin coagulation. The result is the rapid generation of thrombin. Thrombin cleaves fibrinogen and causes it to polymerize to form fibrin, which traps red cells in the clot and supports platelet adhesion. Thrombin also plays critical roles in platelet activation, release of granular contents and irreversible aggregation (Colman, 1993). 


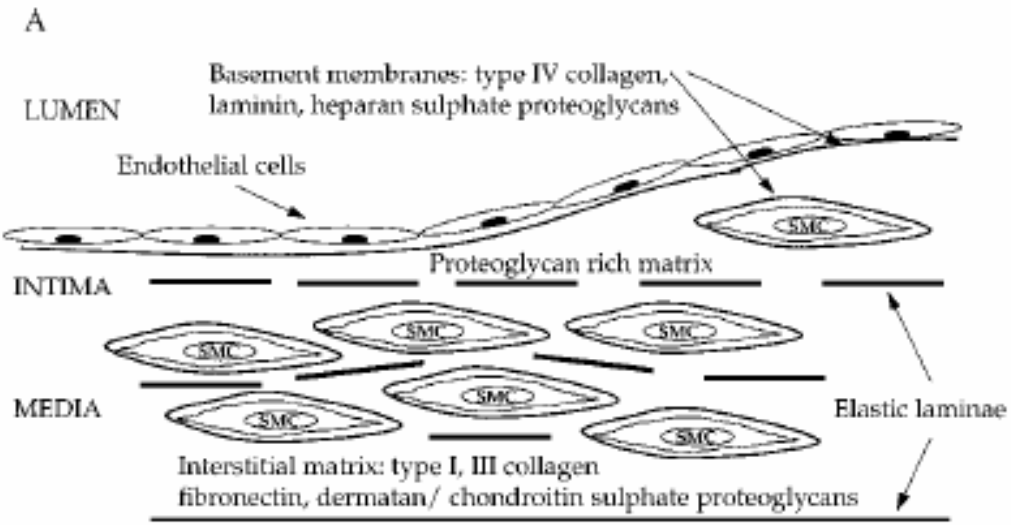

ADVENTITIA: Fibroblasts, fat cells, interstitial matrix

B
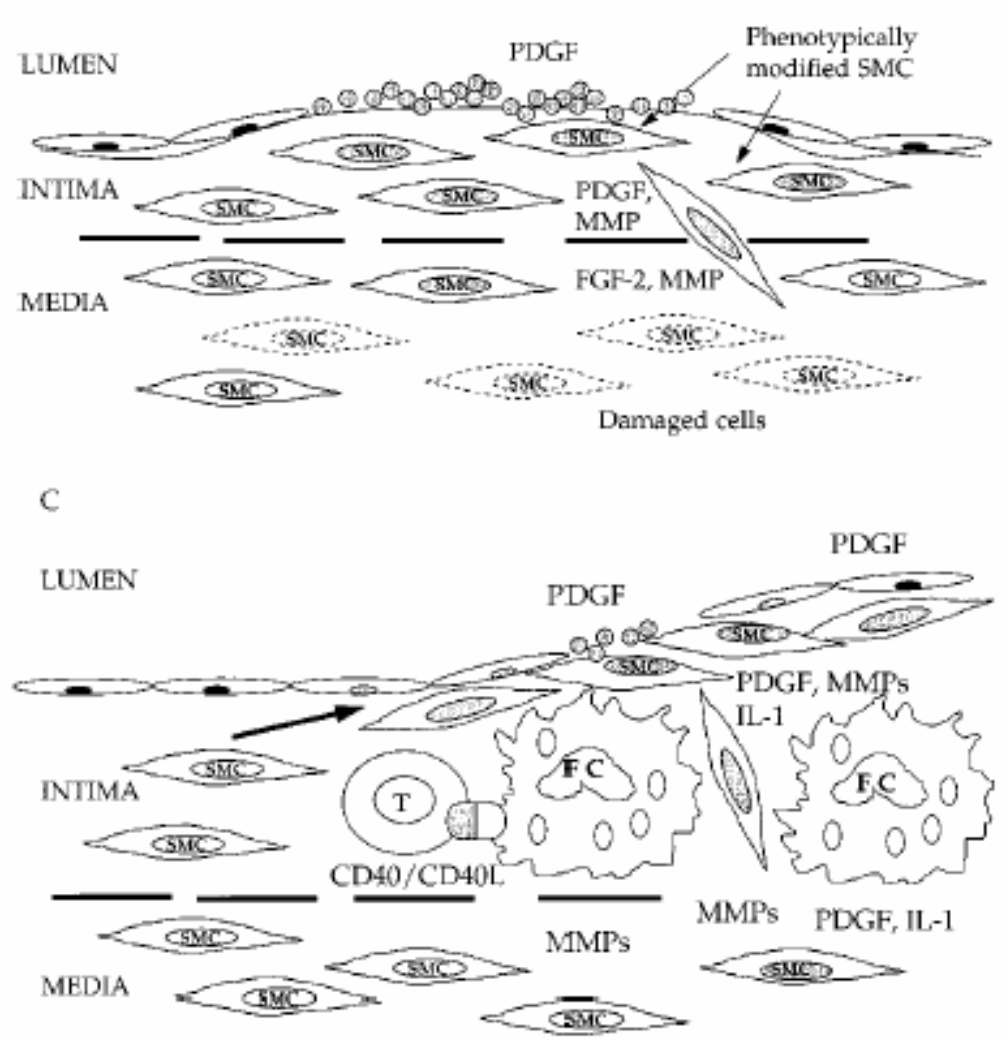

Fig. 2.2 Cellular responses of the vessel wall to injury and inflammation. (A) Normal vessel structure. The figure highlights the three layers of the vessel wall, intima, media and adventitia, as well as different types of extracellular matrix, basement membranes, interstitial matrix and elastic laminae. (B) Response to injury. Platelet-derived growth factor (PDGF) acts as a chemoattractant encouraging phenotypically modified smooth muscle cells (SMC) to migrate from the media into the neointima and replicate there. PDGF also stimulates collegen and proteoglycan synthesis. Metalloproteinases (MMPs) facilitate the migration and proliferation of SMC by remodeling the extracellular matrix.

(C) Response to inflammation. PDGF, activated endothelial cells, SMC, and macrophage foam cells (FC) encourage SMC migration and proliferation (from Newby and Zaltsman, 2000, with permission). 


\subsubsection{Hemodynamic Factors and Correlations to Localization of Lesions}

Atherosclerosis and $\mathrm{IH}$ give rise to focal lesions at reproducible sites around natural or reconstructed bifurcations where abnormalities in hemodynamics are demonstrated (Friedman et al., 1989; Bassiouny et al., 1992; Lemson et al., 2000;). These abnormities include unusual low or high flow, stagnant flow, recirculating flow and flow separation (Crawshaw et al., 1980; Liepsch, 1993; Hughes and How, 1996). As both ahterosclerosis and $\mathrm{IH}$ are the degenerative responses of the vessel wall, several hemodynamic wall parameters have been postulated to quantify the specific effect of impaired hemodynamics, which are magnitude in WSS, OSI, and spatial and temporal gradients in shear.

The low-shear hypothesis of atherosclerosis, first proposed by Caro et al. (1971), has been validated by plenty of subsequent observations and studies (Zarins et al., 1983; Jou et al., 1996; Hofer et al., 1996). Atherosclerotic lesions colocalize with sites of low WSS throughout the arterial tree, from the carotid artery bifurcation to the coronary, infrarenal, and femoral artery vasculatures. For example, Zarins et al. (1983) directly measured atherosclerotic plaque localization in carotid bifurcation at autopsy and compared with flow profiles and shear stress patterns in fluid mechanical models. They found that intimal thickening and atherosclerosis develop largely in regions of relatively low wall shear stress and flow separation. Further supporting this association is the study by Gibson et al. (1993), which, using a serial quantitative coronary angiography technique, revealed that the local rates of atherosclerosis progression in patients with coronary artery disease were correlated inversely with the magnitude of WSS in the condition of controlled systemic risk factors. Low shear stress is also regarded as one of the dominating factors causing intimal thickening in arterial vascular grafts. A quantitative relationship between the amount of shear stress and the associated 
neointimal thickness was established by $\mathrm{Ku}$ and $\mathrm{Zhu}$ (1993). This inverse, nonlinear relationship greatly resembles that seen for early atherosclerotic intimal thickening in arteries. Hofer et al. (1996), again, demonstrated a quantitative correlation between low time-averaged WSS and intimal thickening on the host artery floor and downstream of the anastomosis.

Studies incorporating the pulsatility of the physiological flow found that not only low time-averaged magnitude of WSS but also its directional fluctuations are atherogenic. The representatives of these studies include $\mathrm{Ku}$ et al. (1985) in carotid bifurcation, Asakura and Karino (1990) in human coronary arteries, and Bassiouny et al. (1992) in vascular grafts. For example, Bassiouny et al. (1992) correlated histological data of lesion distributions in canine iliofemoral anastomoses with hemodynamic results from ex vivo flow studies. The schematic description of lesion distribution is shown in Fig. 2.3. They suggested that suture line intimal thickening is probably induced by vascular healing and compliance mismatch, whereas thickening on artery floor, which is not directly traumatized by handling and suturing during surgery and is unrelated to graft type, develops in regions of flow oscillation and relatively low shear. Ku et al. (1985) put forward a non-dimensional parameter OSI to quantify the combined effect of both low magnitude and temporal fluctuations in WSS, based on the finding that the outer wall of the human carotid bifurcation is prone to atherosclerosis where the mean shear stress is close to zero and its direction oscillates over the cardiac cycle. Regions with disturbed flows, such as flow stagnation and low-momentum recirculating flow, usually exhibit higher level of OSI. The close correlation between elevated OSI and increased intimal thickness was further demonstrated in vascular grafts in vivo by Keynton et al. $(2001)$ 


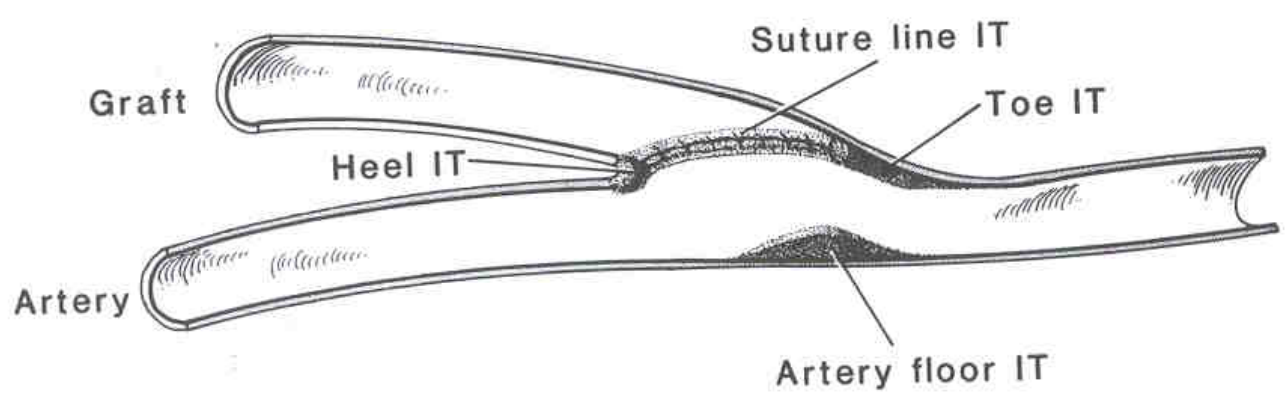

Fig. 2.3 Illustration of sagittal section of end-to-side anastomosis depicts sites of localization of intimal thickening at suture line, toe and heel, and artery floor (from Bassiouny et al., 1992, with permission).

Evidences in high shear theory were generally provided in studies on smaller animals. In cholesterol-fed (Cornhill and Roach, 1976) and low-density lipoprotein (LDL) receptor-deficient (Rosenfeld et al., 1987) rabbits, lesions often develop at regions downstream from bifurcations in larger arteries where the highest shear stress is measured. Also, lesions in ApoE-deficient mice are similar to those in humans, but preferentially at high shear regions (Nakashima et al., 1994). These findings inferred that there must be fundamental differences in vessel wall biology between species that result in different atherogenesis, and high shear stress may affect lesion development in some aspects and this effect exerted on the vessel wall of small species is greater than in humans.

In addition to the hypotheses based on the shear magnitude, an increasing number of studies recognized the importance of the spatial and temporal gradients in WSS. High SWSSG often characterizes the regions with strong secondary or recirculating flow, while high TWSSG perfectly reflects a high degree of pulsatility of physiological flow. The correlation studies for SWSSG and TWSSG are mostly numerical simulations, which present fluid mechanics only and speculate on biological significance. Numerical simulation makes it possible and easier to calculate these complex wall parameters. For 
example, Lei et al. (1995) employed the aorto-celiac junction of rabbits as a representative atherosclerotic model and found that the regions with high spatial shear gradient match the sites susceptible to atherosclerotic lesions. The in vitro studies by Ojha (1993 and 1994) suggested that both high SWSSG and TWSSG may contribute to intimal thickening on the artery floor of arterial graft. Using a photochromic tracer technique to characterize WSS and its gradients, they measured large spatial shear

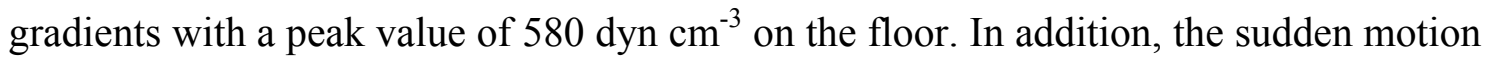
of the stagnation point around peak flow produced sharp temporal shear gradients in the same region with a peak absolute value of $3400 \mathrm{dyn}^{-2} \mathrm{~s}^{-1}$, which is substantially larger than those seen in a straight tube. Thus, the study implied that the development of floor anastomotic intimal hyperplasia might be a response designed to reduce endothelial deformation or injury induced by both shear gradients.

In summary, there are compelling evidences that regions exposed to sustained low WSS and high OSI are predisposed to intimal thickening; however, high WSS and its spatial and temporal gradients may also play certain roles. In regions of strong flow division or flow reattachment, as often seen on the artery floor of distal vascular grafts, low shear stress, high OSI and high shear gradients coexist or reside in close proximity. It is speculated that the cause for lesion development in these regions is multifaceted. Hemodynamic forces exert effects on the vessel wall by triggering a cascade of biological events, and the relationship between hemodynamics and vascular biology would help understand why the disease is located in specific regions and how it is initiated and developed, thereby finding ways to inhibit or mitigate it. The studies on this topic are reviewed in the following sections. 


\subsubsection{Biological Responses of Blood Vessel Wall to Hemodynamic Forces}

The endothelial cytoskeleton is profoundly affected by shear stress. The actin and microtubule cytoskeletons, in particular, are essential in maintaining the integrity of the vascular endothelium and regulating endothelial repair following endothelial disruption or denudation due to cell loss. Actin microfilaments are arranged centrally in endothelial cells to form stress fibers, and peripherally to form a dense peripheral band. The dense peripheral band plays a role in cell-cell adhesion and thus may be important in regulating cell permeability. Endothelial cell adaptions to high shear include thicker and longer central microfilaments and disrupted dense peripheral band (Langille, 1995), and may contribute to increased cell permeability. These changes are reversible based on an intact endothelium. Morphological changes in the endothelial cells is one of the reassembly responses following partial disassembly of the adherens junction complex induced by shear stress. Steady laminar flow tends to align the endothelial cells with the flow field and gives the cells an elongated elliptical configuration. The in vitro experiment concerning shear stress and EC morphology conducted by Okano and Yoshida (1993) suggested that ECs exposed to low WSS exhibits wide elliptic or polygonal shape, in contrast to the elongated cells in high shear regions. They suspected that endothelial cells in low shear regions might be functionally activated, which imparts vulnerability on these regions to lipid deposition. In vivo, the disease-prone regions are often characterized by disorganized ECs, decreased peripheral actin, and increased fiber numbers with attached monocytes. A study in rabbits revealed that the circumferential axis of the flow divider in aortic intercostals ostia was covered by two or three rows of rounded cells followed by the well-aligned cells. Such rounded and irregular-shaped cells were present also in flow separation and reattachment regions (Nerem et al., 1986). However, whether these shape changes are significant or 
irrelevant to the disease development has not been demonstrated, and there are also different views about these changes. For example, Davies et al. (1995) characterized the surface topology of the endothelial monolayer by a novel application of atomic force microscopy and correlated it with the results of numerical simulations. They found that the flattening of the cell surface caused by shear stress imposed on the apical membrane reduces local shear gradients, which suggested that changes in cell morphology caused by flow may be an adaptive response to maintain endothelial integrity and signaling between adjacent cells and these morphologic adaptions may be compromised at locations where local flow disturbances exist. High spatial shear gradient would cause severe local deformation and trauma to the apical cell memebrane and associated structures (DePaola et al., 1992; Truskey et al., 1995; Satcher et al., 1991), therefore breaking down consistent communication between cells and resulting in elevated wall permeability to macromolecules (Lei et al., 1995; Buchanan et al., 1998), which are transported through the intercellular junction or by vesicles to the subendothelium. As demonstrated by the study of Truskey et al. (1995), endothelial cells at the reattachment point were subject to severe morphological and functional changes and migrate away due to cell detachment, a minimum cell density and a maximum percentage of labeled nuclei, and the maximum spatial shear gradient coincides with this region.

Physiologic shear stress has also been shown to be necessary for timely repair of the mechanically injured endothelium. Within in vivo mechanical injury models, low shear substantially inhibits endothelial repair by inducing impaired cell-cell contacts and disordered alignment of the cytoskeleton in cells along the wounded endothelium (Vyalov et al., 1996). Disturbed repair may not only promote atheroma formation through enhanced endothelial permeability as a result of endothelial injury but may also 
lead to the formation of endothelial erosions and fissures that are associated with complicated atherosclerotic lesions (Dickson and Gotlieb, 2004).

Altering shear stress has acute effect on intimal proliferative state as demonstrated by Kohler and Jawien (1992) using well-controlled experimental models. A reduction in flow and shear stress results in greater intimal proliferative responses to balloon injury of rat carotid arteries than in high flow rates. Low shear stress is also shown to induce endothelial cell loss and desquamation, altered morphology, a decrease in the number of actin stress fibers and an increase in peripheral actin straining (Walpola et al., 1993). Furthermore, smooth muscle cell proliferation involved in the pathogeneses of both atherosclerosis and $\mathrm{IH}$ is likely to be stimulated by endothelial cell factors that are regulated by shear stress. Kraiss et al. (1996) showed that low shear stress results in higher levels of endothelial PDGF-A, mRNA and greater smooth muscle cell proliferation in an arteriovenous fistula model of altered flow in baboons. Contrarily, elevated shear leads to increased expression of endothelial nitric oxide synthase (eNOS), a lower degree of neointimal and smooth muscle proliferation, and regression of previously established neointima. An inverse relationship between shear stress and the degree of intimal proliferation was also demonstrated in rodent experiments (Rieder et al., 1997). In addition, chronically increasing blood flow caused an increased content of cyclic quanosine monophosphate in the arterial wall, indicating that high flow and high shear stress can chronically activate the NO pathway, a pathway important for the regulation of cell function and communication (Arnal et al., 1993). All these findings provide biological evidences for the detrimental effects of low shear stress. Oscillatory shear stress (or temporal variations in shear), unlike steady shear stress can disrupt the atheroprotective molecular pathways by decreasing eNOS expression (Ziegler et al, 1998). Once the atheroprotective role of the endothelium has been compromised, a 
vicious cycle of vascular wall injury causing atherosclerotic plaque formation and subsequent disruption in blood flow can promote atheroma progression and eventually atherosclerotic lesion development. An in vitro cell culture study from Bao et al. (1998) recognized the distinct roles of the temporal component of shear in atherogenesis. They demonstrated that temporal gradient in shear rather than steady shear stress is a potent stimulus for the expressions of the atherogenesis-related gene PDGF-A and MCP-1.

In summary, hemodynamic forces induces critical and diverse biological responses of the cells in blood vessels. Responses to low flow and low shear stress include increased aggressiveness in endothelial cell morphology, endothelial cell loss and desquamation, greater intimal and smooth muscle cell proliferation, higher levels of PDGF-A protein and mRNA, reduced production of $\mathrm{NO}$ and PGI2, as well as disturbed repair of injured endothelium. The negative effect of high shear stress and its gradient is usually associated with regression and increased permeability of the endothelium. The temporal variations in shear, however, may disrupt the atheroprotective molecular pathways. Each of these hemodynamic factors influences in a certain way the pathogeneses of vascular diseases. However, to clarify the dominating ones need more detailed biological and correlation studies equipped with advanced technologies that would allow noninvasive shear measuring and lesion characterization in vivo.

\subsubsection{Shear-dependent Transport of Blood Species}

Transport of blood species (e.g. LDL, platelet, leukocyte) and dissolved gases $\left(\mathrm{O}_{2}, \mathrm{CO}_{2}\right)$ in the arterial lumen and through the arterial wall have long been indicated as important events in the disease development. Both processes are coupled and strongly influenced by local hemodynamic environment. Deng et al. (1995) investigated the effect of nearwall blood flow velocity and plasma filtration velocity across the arterial wall on 
luminal surface concentration of low-density lipoproteins (LDL) and the uptake of tritium-cholesterol. They found that the LDL surface concentration increased inversely with wall shear rate, and increased LDL concentration would promote the rate of lipid infiltration into the blood vessel. A more detailed study concerning LDL concentration at the luminal surface was conducted by Wada and Karino (2002) in steady flow conditions. It was shown that in the regions distal to the apex of the inner wall of each bend, elevated LDL concentration co-localizes with stagnant flow and relatively low wall shear stress. However, the highest LDL concentration does not necessarily occur in the sites of the lowest shear stress, and instead it is found in the regions with slow secondary and recirculating flows, indicating that locally elevated surface concentration is caused not only by low shear stress but also by near-wall flow patterns which determine the paths and residence time of fluid elements. These findings suggest that even in normocholesterolemic subjects, locally hypercholesterolemic environment is still likely present in certain regions, which favor the occurrence of intimal thickening. The LDL concentrated at the luminal surface would be further transported into the inner layer of the vessel wall, and this process is suggested to be promoted by increased permeability of the endothelium. Berceli et al. (1990) found that LDL incorporation in the rabbit aorta-iliac bifurcation was elevated in the lateral wall that is subject to flow reversal. They explained that the higher LDL incorporation in the flow reversal conditions might be caused by enhanced macromolecule permeability as a result of high shear gradient. The possible relationship between high spatial shear gradient and elevated wall permeability to LDL was also suggested by Lei et al. (1995) by simulating LDL transfer in a model with geometry representing the rabbit aorticiliac junction. Taken together, the above studies exhibit a general picture that zones of 
abnormal shear stress or flow patterns, elevated LDL concentration and influx tend to colocalize with each other, and with susceptible areas of lesion development.

Leukocyte adhesion and transmigration of monocytes and leukocytes into the blood vessel wall are regulated by endothelial cells by secretion of chemotactic factors and expression of cell surface molecules. ICAM-1 binds $\beta 2$-integrins on various white blood cell derivatives, while VCAM-1 mediates adhesion of monocytes to the endothelium (Cybulsky et al., 1991). VCAM-1 is higher in the vicinity of atherosclerotic plaques and is one of the earliest signs for fatty streaks (Cybulsky et al., 1991). An in vivo study by Walpola et al. (1995) showed that the reduction in shear stress enhances VCAM-1 expression by 30 times as compared to the controlled shear stress, and ICAM-1 expression fell to approximately $30 \%$ of the controlled shear. High shear stress, however, was shown to increase both VCAM-1 and ICAN-1 expressions by 3.5 times and 1.6 times of the controlled shear, respectively. It is noteworthy that extensive monocyte adhesion occurs in low shear conditions with enhanced VCAM-1 expression, whereas no sign of monoyte adhesion is observed in high shear conditions. Therefore, they suggested that leukocyte binding in vivo was promoted by low shear stress, which is consistent with their earlier observation that early stage of atherogenesis is characterized by subendothelial accumulation of monocytes, and low shear stress alone can stimulate this process (Walpola et al., 1993).

Platelets, as another type of monocytes, are closely involved both in the early development of atherosclerosis and in the events that lead to acute thrombosis as unstable angina pectoris and acute myocardial infarction. Thrombosis is triggered when a thrombogenic surface is exposed to blood. Thrombogenic surfaces include the subendothelial and medial layers of blood vessels, the subendothelial components of 
atherosclerotic lesion such as fibrous plaque cap and atheromatous core as well as most artificial surfaces (Fernandez-Ortiz et al., 1994). The first stage of thrombogenesis is platelet adhesion on a surface, which is then followed by platelet activation, aggregation and the formation of mural thrombi. Platelets can be activated by any surgical injury or atherosclerotic plaque rupture releasing a cloud of chemicals. Extremely high shear stress is also likely to activate platelets with short exposure (Holme et al., 1997). In addition, convection patterns and high residence time in the regions with separated flow, flow stagnation or recirculation are shown to induce both platelet activation and aggregation (Bluestein et al., 1997; Bluestein et al., 1996). Furthermore, a strong direct correlation between shear rate and platelet and fibrin deposition was established by Ouriel et al. (1991). Platelet aggregation on collagen-containing stenotic surfaces is highest at the stenosis throat where shear rate is highest (Badimon et al., 1986). However, the situation is shown to be otherwise on smooth artificial surfaces. It has been found that on artificial surfaces platelet aggregation was maximum in observed areas of flow recirculation and low shear and minimum in locations of high shear (Bluestein et al., 1997; Schoephoerster et al., 1993).

Although the role of hemodynamics in the transport and deposition of macromolecules and monocytes is still unclear, it is widely held that low shear stress and disturbed nearwall flow patterns increase surface concentration of LDL and promote leukocyte binding into the vessel wall, and LDL influx is enhanced by increased endothelial permeability due to high shear gradient. The response of platelets to blood flow is dependent on the surface property of the vessel. Increasing shear rate on collagencontaining blood vessels generally enhances platelet adhesion, whereas on artificial surfaces platelet adhesion is depressed by high shear and favored by low shear and recirculating flow. 


\subsection{CURRENT EFFORTS ON GEOMETRIC OPTIMIZATION OF DISTAL ANASTOMOSES}

In recent years, many researchers have recognized that anastomotic geometric factors, especially in the distal part, play critical roles in determining local mechanical environment and thus long-term bypass graft patency. This has stimulated much work, both experimentally and numerically, to explore these roles, most of which is focused on the peripheral anastomoses. In the following, recent studies as regards the effects of several primary geometric factors are reviewed.

A number of studies supported that a graft that is larger than the artery is favorable. For example, Lei et al. (1997) via numerical analysis suggested that a 1.6:1 graft-to-artery diameter ratio optimizes hemodynamics in terms of reduced spatial shear gradient. Jian et al. (1999) studied the effect of diameter ratio between bypass and blood vessel on anastomosis based on various nonlinear stress-strain laws with numerical analyses. They concluded that under normal physiological blood pressure, if the initial diameter of the bypass with high rigidity is kept 1.2 1.4 times larger than that of the blood vessel, stress concentration at the anastomosed site, on average, can be reduced during a pulsatile pressure cycle. However, an in vivo study carried out by Keynton et al. (1999) in canines revealed negative effects of large diameter ratios. They concluded that wall shear rates within end-to-side distal graft anastomoses, particularly along the artery floor, increase sharply when graft-artery diameter ratio increases from 1.0 to 1.5 , and thus may play a role in the development of IH through local alteration of mass transfer and mechao-signal transduction within the endothelium.

As regards the graft-artery angle, a majority of studies favor the smallest angle for it produces a more streamlined flow field with less flow separation at the toe, less shear variations on the floor, as well as reduced spatial shear gradient (Taylor et al., 1992; Lei 
et al., 1997; Moore et al., 1999; Hughes and How, 1995; Song et al., 2000). However, Fei et al. (1994), by simulating anastomotic angles of $20^{\circ}, 30^{\circ}, 40^{\circ}, 45^{\circ}, 50^{\circ}, 60^{\circ}$ and $70^{\circ}$, found an adverse effect exerted by acute angles that smaller angles result in low mean shear stresses on the artery floor opposite the graft orifice where intimal thickening usually occurs. In addition, both large and small angles are shown to induce significant morphological changes in vivo as reported by Jackson et al. (2001). They reported that the anastomoses with acute angles $\left(10^{\circ}\right)$ exhibited intimal proliferation on the artery floor, while the floor of the right-angle anastomoses were characterized by wall atrophy with both cell loss and matrix degradation.

For below-knee arterial reconstructions, there are clinical evidences that interposition vein cuffs and patches have obviously increased graft viability (Miller et al., 1984; Stonebridge et al., 1995; Morris et al., 1993; Taylor et al., 1992). The configurations of the Miller cuff and Taylor patch are shown in Fig. 2.4. The theories on what causes the beneficial effects have been controversial. Some researchers attributed the benefits to modified anastomotic geometry, which results in improved hemodynamics (How et al., 2000; Wijesinghe et al., 1999; Harris and How, 1999; Da Silva et al., 1997; Perktold et al., 2002). For example, How et al. (2000) using laser Doppler anemometer demonstrated a cohesive and unidirectional vortex within the cuff that is persistent from systole to the end of diastole. They suggested that this vortex might have a rush-out effect on the vessel wall and might be responsible for a favorable redistribution of WSS in the cuffed anastomosis. The redistribution of WSS removes intimal thickening at critical sites (e.g. on the artery floor and along the distal native artery) to the upstream regions with a more accommodating area. A recent numerical study of Cole et al. (2002a), however, showed worsened anastomotic hemodynamics when the cuff is employed. Sharp WSS variations on the artery floor and significant flow separation 
distal to the cuff toe were observed. They suggested that the benefits associated with the cuff is probably associated with the intrinsic biological properties of the vein. Another study of Cole et al. (2002b) compared the Taylor patch with the conventional anastomosis. It was found that the Taylor adaptation with hemodynamically smoother transitions and a smaller angle between the graft and artery gives rise to reduced flow separation at the toe and more favorable shear stress distribution on the artery floor and $\mathrm{IH}$ in these regions would be alleviated accordingly. In addition, for both Miller cuff and Taylor patch, greater anastomotic area resulting in a lower resistance and easier execution of the anastomosis causing less technical errors particularly for small-caliber below-knee vessels (e.g. popliteal and crural arteries), are also part of the reasons for the improved clinical results (Taylor et al., 1992; Stonebridge et al., 1995).
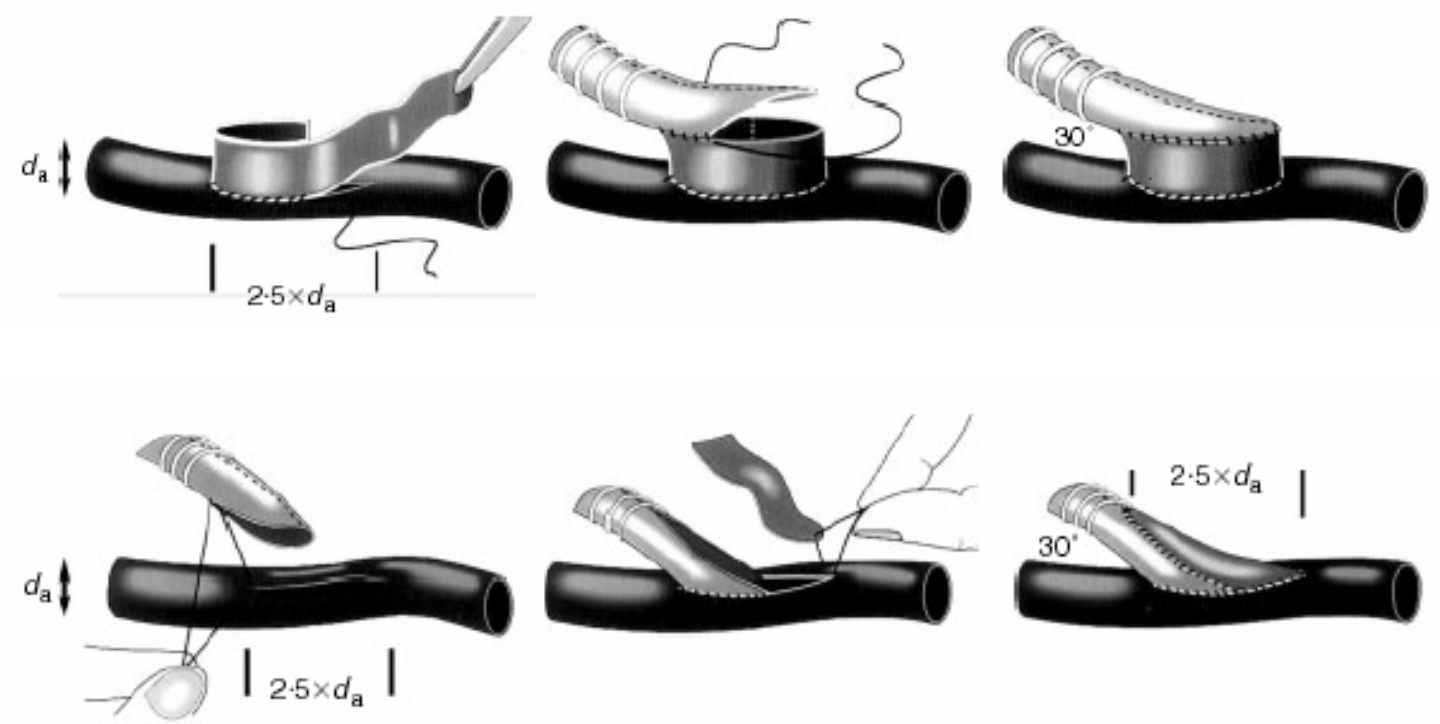

Fig. 2.4 Two methods of juxtaposing vein between PTFE graft and artery at the distal anastomosis: Miller cuff (top) and Taylor patch (bottom) (from Trubel et al., 2004, with permission).

Nonplanarity is a primary feature of the anastomosis. It was shown by Sherwin et al. (2000) that nonplanar three-dimensional inflow substantially reduces the peak WSS magnitude by approximately $10 \%$ in comparison with the planar case in steady flow 
conditions. Successively, Papaharilaou et al. (2002) studied the same models in pulsatile flows. In the non-planar model, the spatial distribution of WSS is significantly altered and the time-averaged peak WSS magnitude and OSI magnitude are reduced by $10 \%$ and $22 \%$, respectively, as compared to planar model. Moreover, the stagnation point follows a parabolic path in the non-planar model in contrast to a straight-line path in the planar model. It is therefore implicated that the changes in the distributions of WSS and OSI introduced by nonplanar inflow geometry may alter the localization and severity of IH lesions.

In addition, the dimensions of the hood are also suggested to have certain effects on anastomotic flows. A longer hood, as reported by Lei et al. (1997), would reduce spatial shear stress gradient and result in less flow skewing in the host artery. However, the long hood may cause unfavorable effects in the case of small distal outflows. White et al. (1993) examined the hemodynamic patterns in two models of end-to-side anastomoses with different hood lengths. The results showed that there is a clear tendency for the longer hood length to undergo flow separation along the distal segment of the hood, specifically when the outflow portion in the distal artery is less than $50 \%$, and the separation does not occur for the cases of larger outflows in the distal artery.

Summarizing the above, geometric factors are important in regulating anastomotic hemodynamics. The significance and specific roles of each factor may vary under different flow conditions and between different vascular systems. More detailed investigations are required to explore this field, especially for the coronary anastomoses. 


\section{CHAPTER 3}

\section{NUMERICAL METHOD AND THEORY}

\subsection{INTRODUCTION}

Arterial hemodynamics can be studied in a variety of ways, including laboratory experimentation using excised vessel segments or glass/plastic models, in vivo experimentation, analytical solution as well as numerical simulation. Each approach has its particular advantages and disadvantages. The most commonly used methods for engineers would be in vitro bench-top study and numerical modeling. The early in vitro experimental approaches mainly include flow visualization and dye studies, which are limited to provide qualitative information of global flow field (White et al., 1993; Ethier et al., 1998a). Recently, noninvasive techniques using optical probes for quantitative velocity measurements have been developed and experienced much improvement, such as the laser-Doppler anemometry (LDA) and the particle imaging velocimetry (PIV). These techniques offer powerful tools for obtaining detailed flow characteristics in real-size or scaled-up physical models (Lei et al., 2001; Bates et al., 2001; Heise et al., 2003). However, the experimental study is more expensive and has less flexibility in parametric studies as compared to numerical simulation. Since the present study involves investigating effects of altering geometric parameters in a wide range and calculating hemodynamic parameters, the advantages of the numerical approach become obvious. In addition to its flexibility in parametric studies, numerical approach can provide sufficient spatial data resolution, especially in the near-wall region, which allows the accurate calculations of complex wall parameters. 
In this chapter, the numerical simulation method used to solve the basic equations governing the blood flow is described. Prior to the study, a demonstration of mesh independence is performed in order to ensure an adequate accuracy of the numerical method.

\subsection{MESH GENERATION AND DEMONSTRATION OF MESH INDEPENDENCE}

Insufficient spatial resolution is the most common cause for failing computational meshes in solving three-dimensional problems, leading to inaccurate calculations of local velocity field and derived lower-order parameters. Therefore, demonstration of mesh-independence is required as a necessary (but not sufficient) condition for the validity of a computational result (Freitas, 1993; Prakash and Ethier, 2001). Mesh independence means that the converged solution obtained from numerical simulation is independent of the mesh topology and density. Increasing the number of cells in one region or throughout the entire domain for a mesh-independent CFD model would not (ideally) change the flow-field solution and integrated quantities.

The demonstration of mesh independence was first carried out in testing two meshes with different cell types. The topologies of these meshes are illustrated in Fig.3.1, which contain 16,835 hexahedral cells and 48,415 tetrahedral cells, respectively. The meshes were generated using the Gambit mesh generation package (Fluent Inc., Lebanon, NH). Considering the symmetry in the models, only half of the domain was calculated. The results of WSS distribution on the model surface calculated by the two meshes are also displayed in Fig. 3.1. It is clearly shown that the WSS contour calculated from the hexahedral mesh is very definitive in characterizing areas of different WSS levels, whereas the tetrahedral mesh has a highly-diffusive contour with 
ill-defined boundaries between different areas. Direct comparison of cycle-averaged WSS on the artery floor in Fig. 3.2 also shows that finer spatial resolution of data is associated with the hexahedral mesh as compared to the case of tetrahedral mesh. In addition, the number of cells in the tetrahedral mesh is almost three folds as much as that in the hexahedral mesh, which potentially increases the computing time. Therefore, this comparison has demonstrated a superiority of hexahedral cells to tetrahedral cells in calculating unsteady arterial blood flows for the current study.

Mesh independence was further demonstrated using a sequence of hexahedral meshes of increasing density (Fig. 3.3). An initial solution was computed on a coarse mesh containing 15,448 hexahedral cells (Mesh 1). By increasing the number of cells over the entire domain, finer mesh series were then obtained with 68,618 (Mesh 2), 108,936 (Mesh 3) and 252,592 (Mesh 4) hexahedral cells. For each mesh, an increased cell density was imposed at the anastomotic region where most complex flow environment is anticipated. In most arterial flow studies, the most important variables of interest are flow velocity field, shear stress and its derivatives such as the spatial and temporal gradients. Shear stress and its gradients are lower-order computed variables than velocity field. Therefore, a mesh considered well-resolved for calculating velocity field does not necessarily imply accurate WSS results. In this study, distributions of WSS and its spatial gradient rather than velocity field were compared between the successive meshes. The comparison included both a qualitative comparison of unsteady WSS contour, and a quantitative comparison of spatial shear gradient variation along the floor. A mesh independence solution is considered to be attained when no appreciable difference in WSS contour exists between successive meshes and the largest relative error in shear gradient was within 5\% (Prakash and Ethier, 2001). 
Figure 3.4 shows the WSS distributions over both space and time on the floor stagnation region for each mesh. The floor stagnation region, which is most notorious for the disease development, is characterized by drastic WSS variations. A general agreement exists in the WSS contour for all the meshes since the basic features are similar. For example, surrounding the stagnation point (where WSS is zero) are regions of the highest and lowest WSSs, and the WSS changes more prominently during the diastolic phase, especially at the peak flow. However, detailed examination reveals that there is still appreciable difference present between the two coarser meshes and the other two finer ones. The area with the highest WSS in Mesh 1 and 2 is slightly larger in size and the area with the lowest WSS is smaller. The maximum WSS magnitude from Mesh 1 to 4 is 6.27, 5.83, 5.67 and 5.59, respectively. It is suggested that a mesh with insufficient resolution tends to overestimate the overall WSS level. Considering the solution obtained on the finest mesh as a "gold standard", the relative errors ( $\left|\left(\tau_{i}-\tau_{4}\right) / \tau_{4}\right| \times 100 \%$, where $i$ is the mesh serial number and $\tau_{4}$ is the WSS for Mesh 4.) for Mesh 1 to 3 are calculated to be $12.2 \%, 4.3 \%$ and $1.4 \%$, respectively.

Direct comparison of cycle-averaged spatial shear gradient is shown in Fig. 3.5. Mesh resolution have a more significant effect on the derived quantity of shear stress. Distinctively higher magnitude is associated with the coarsest mesh. The gradient is also appreciably higher in Mesh 2, as compared to the two finer meshes which show a perfect match between themselves. The largest relative errors in spatial shear gradient on the floor for Mesh 1 to 3 are 19.5\%, 6.6\% and 2.2\%, respectively.

This study has demonstrated that the meshes with 15,448 and 68,618 cells clearly do not give mesh-independent results in terms of WSS and its spatial gradient, and mesh independence is established at 108, 936 cells. Further mesh refinement (from 108,936 
cells to 252,592 cells) yields only small, insignificant change in all the variables examined above. The mesh of 108, 936 cells represents a trade-off between accuracy of results and computational costs, and therefore this mesh density was used for all production runs with modest errors (within 5\%) to be expected in the results. The mesh required for a mesh-independent solution in the present study contains a higher density as compared to a number of studies in the computational hemodynamics literatures (Cole et al., 2002a,b; Kute and Vorp, 2001; Lei et al., 2001). It is probably because that the mesh independence study in the literatures has been performed in steady flow conditions, while the present study is based on unsteady physiological flows. The unsteadiness would certainly complicate the computation and require higher spatial resolution in meshes. Besides, a higher judging criterion based on shear gradient is used for the present study. The shear gradient is the derivative of shear stress and second derivative of velocity; therefore, an accurate shear gradient field needs a higher mesh resolution than an accurate WSS or velocity field does. 

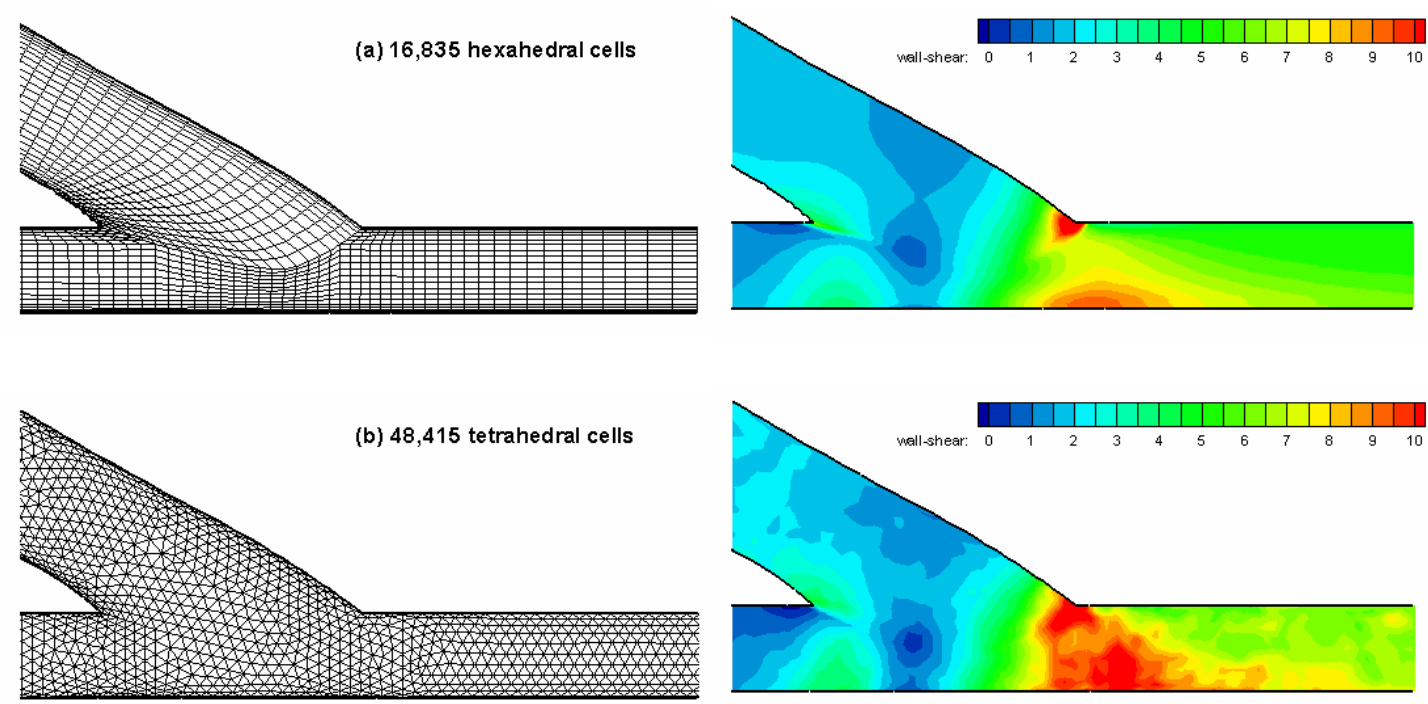

Fig. 3.1 Mesh topologies (left) and WSS distributions (right) on the model surface for the two meshes with hexahedral and tetrahedral cells.

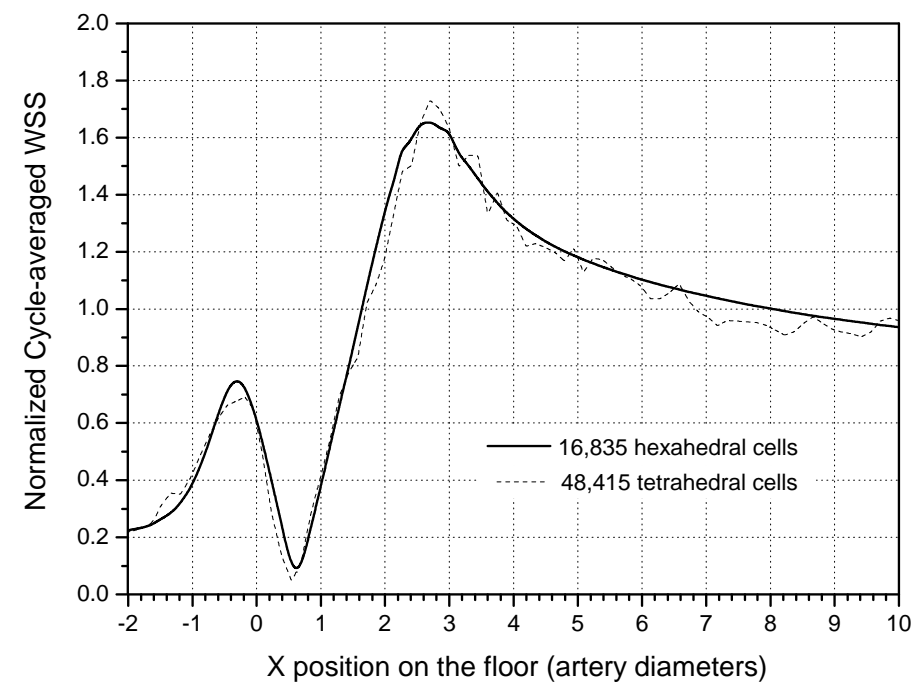

Fig. 3.2 Direct comparison of cycle-averaged WSS on the artery floor between the two meshes with hexahedral and tetrahedral cells. 

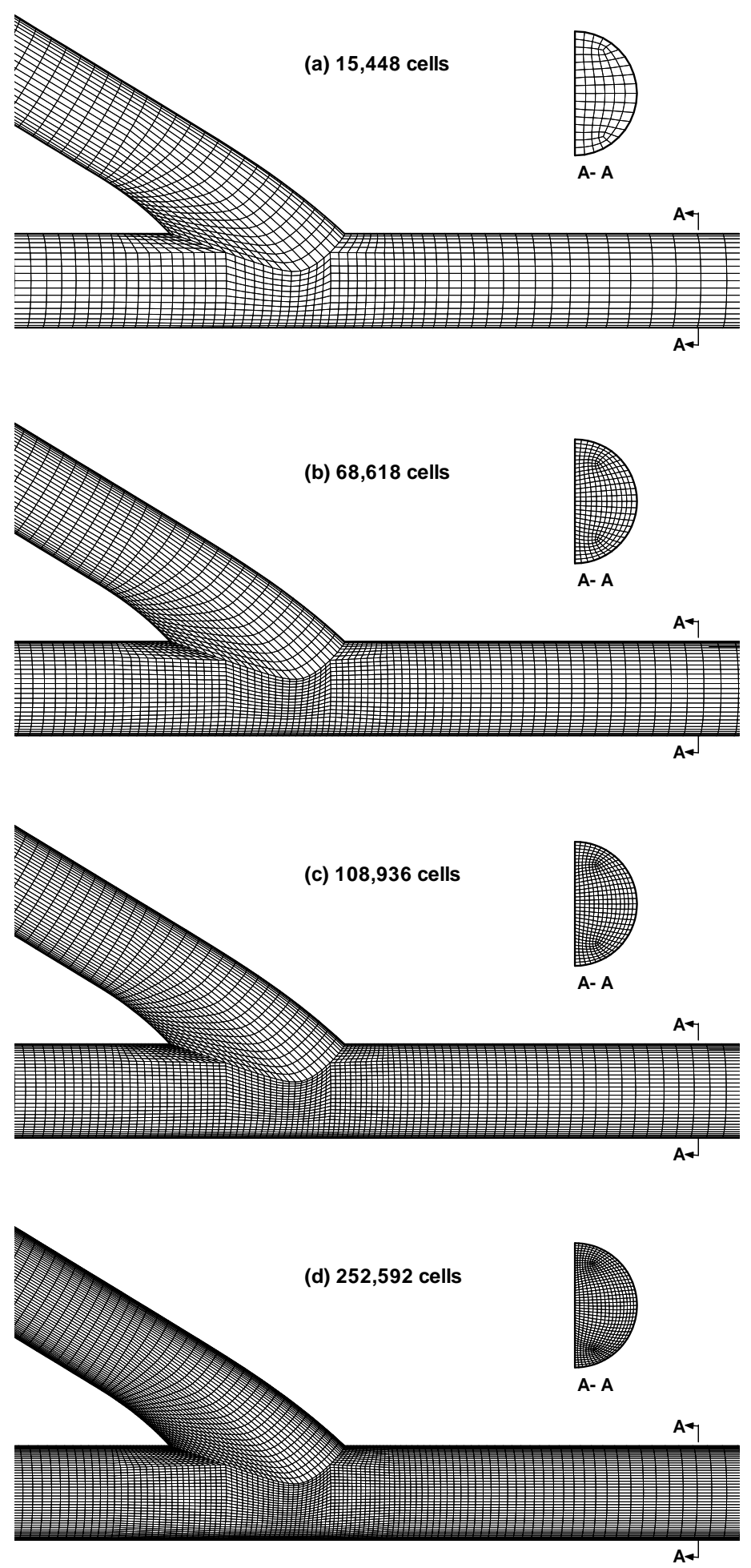

Fig. 3.3 Topologies of the hexahedral meshes of increasing density. 

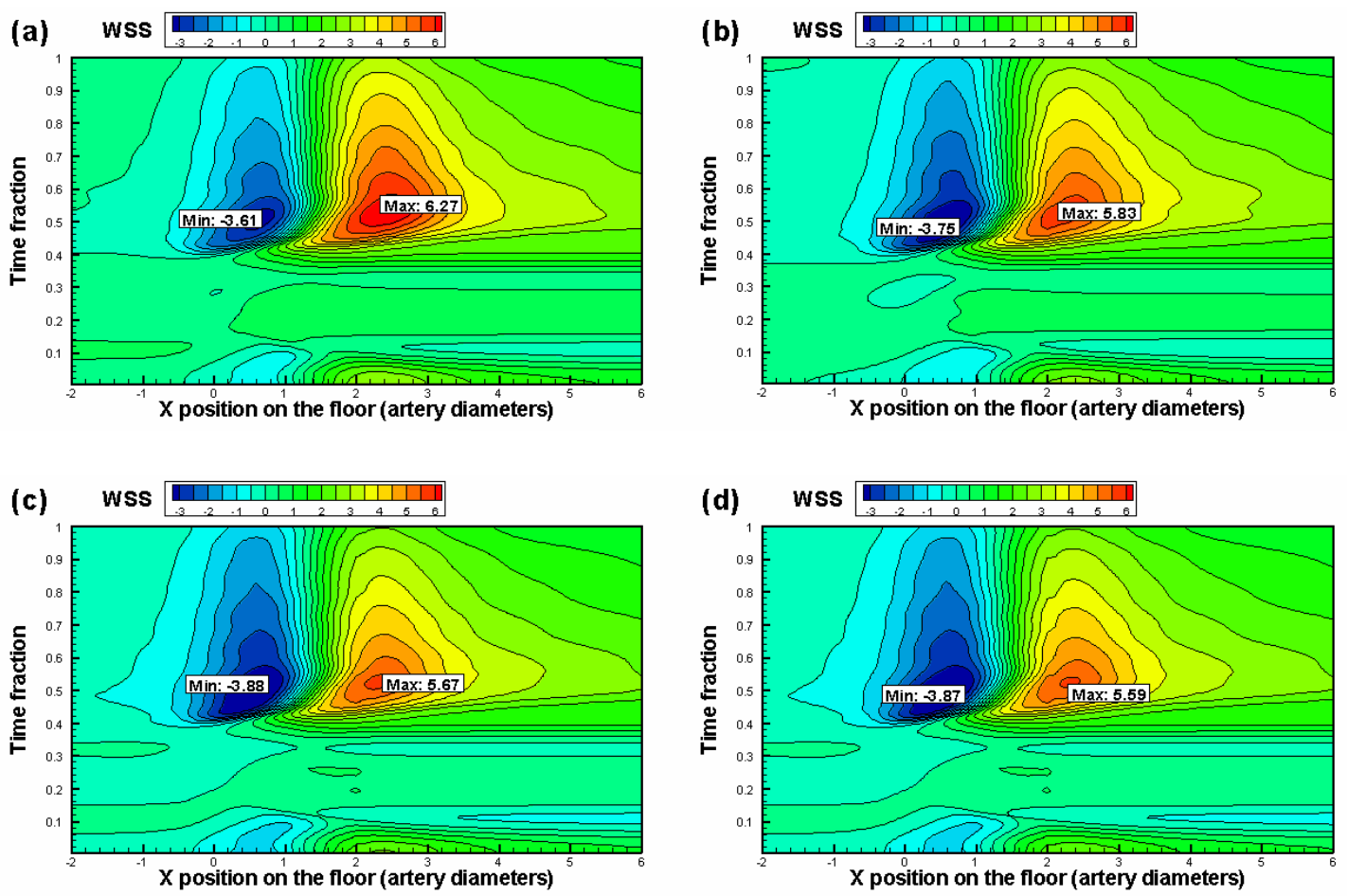

Fig. 3.4 WSS variation on the floor over both space and time for the meshes with (a) 15,448 cells, (b) 68,618 cells, (c) 108, 936 cells, and (d) 252, 592 cells.

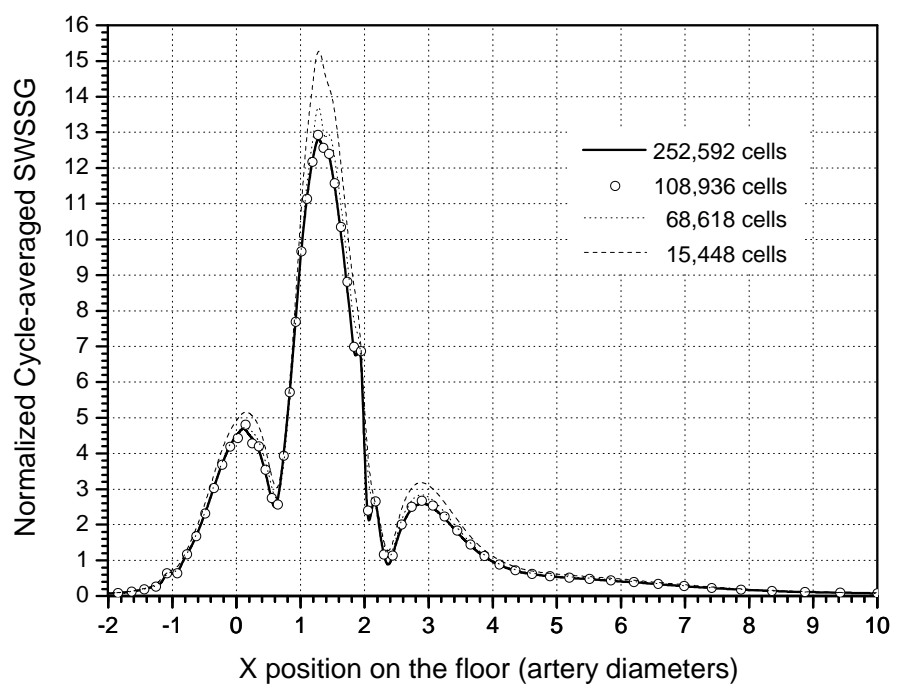

Fig. 3.5 Comparison of mean SWSSG on the floor between the four meshes. 


\subsection{NUMERICAL METHOD}

Numerical simulation has proven to be a practical and reliable tool for investigating the time-varying, three-dimensional vascular flows. The parameters and procedures to perform the simulations for the present study are described in the following.

\subsubsection{Governing Equations and Flow Conditions}

The blood was assumed as incompressible, homogeneous and Newtonian fluid with a dynamic viscosity of $0.004 \mathrm{~kg} \mathrm{~m}^{-1} \mathrm{~s}^{-1}$ and a density of $1100 \mathrm{~kg} \mathrm{~m}^{-3}$ (Patel and Vaishnav, 1980). Realistically, the human blood has a non-Newtonian, shear-thinning nature because of the presence of the red cells. However, Thurston (1979) found that the nonNewtonian behavior of blood is important only when the shear rate is lower than $100 \mathrm{~s}^{-1}$. Since the lowest computed mean shear rate in the coronary artery is around $586 \mathrm{~s}^{-1}$, the non-Newtonian effects are not expected to be important in the coronary arteries. It was further evidenced by Friedman et al. (1992) that non-Newtonian fluid leads to no significant changes in the correlation of vascular disease to wall shear distributions. Specifically, the velocity profiles are affected by flow rate and hematocrit, particularly in vessels $<0.5 \mathrm{~mm}$ diameter (Goldsmith and Turitto, 1986). As the blood vessels investigated in the studies of this thesis are either equal to or larger than $2 \mathrm{~mm}$ in diameter, the non-Newtonian effect should be minor and thus was ignored. In addition, the graft and arterial walls were assumed to be rigid. Although the influence of the elastic property of blood vessels on certain aspects of the blood flow, e.g. flow patterns and WSS, has been noted (Wang and Tarbell, 1995; Nichols et al., 1977), it is generally considered to be minor, and the rigid-wall assumption is shown to be reasonable for arterial hemodynamics in peripheral artery studies (Friedman et al., 1992; Steinman and Either, 1993; Duncan et al., 1990; Deters et al., 1986). Since coronary arteries are less 
elastic than peripheral arteries (Perktold and Rappitsch, 1995) and a venous graft is found to lose its elasticity as soon as one week post operation due to medial thickening as a result of arterialization (Dobrin et al., 1988), wall elasticity is expected to be even less significant here.

Based on the above assumptions, the unsteady, isothermal, laminar blood flow is governed by the full time-dependent Navier-Stokes equations. Using normalized nondimensional variables, the governing flow equations can be written as follows:

$$
\begin{gathered}
\nabla \cdot \vec{u}=0 \\
\frac{\partial \vec{u}}{\partial t}+(\vec{u} \cdot \nabla) \vec{u}=-\frac{1}{\rho} \nabla p+v \nabla^{2} \vec{u}
\end{gathered}
$$

Here Eq. (3.1) is the mass conservation equation or continuity equation and Eq. (3.2) the momentum conservation equation. $\vec{u}=\left(u_{x}, u_{y}, u_{z}\right)$ is the velocity vector, $t$ the time, $p$ the static pressure, $\rho$ the blood density, and $v$ the kinetic viscosity.

Fully developed pulsatile velocity profiles were applied at the graft inlet. At outflow boundaries, the conditions describing zero surface traction were assumed. The mathematical description of this condition takes the form:

$$
-p \vec{n}+\frac{1}{\operatorname{Re}} \frac{\partial \vec{u}}{\partial \vec{n}}=0
$$

where $\vec{n}=\left(n_{1}, n_{2}, n_{3}\right)^{T}$ denotes the outward pointing normal unit vector at the outflow boundary. This boundary condition is used to model flow exits where the details of the flow velocity and pressure are not known prior to the solution of the flow problem. Non-slip condition was applied at all walls.

\subsubsection{Solution Procedure}


The transient incompressible three-dimensional Navier-Stokes equations governing the fluid motion were solved based on a segregated iterative algorithm involved in Fluent ${ }^{\circledR}$ software (Fluent Inc., Lebanon, NH, USA), a control-volume-based commercial code. This control volume technique consists of integrating the governing equations about each control volume, yielding discrete equations that conserve each quantity on a control-volume basis. Applying Finite Volume method, the continuity and momentum equations (3.1) and (3.2), when integrated over a constant volume $V$ enclosed by the surface $A$, take the integral forms:

$$
\begin{gathered}
\oint \vec{u} \cdot d \vec{A}=0 \\
\frac{\partial}{\partial t}(\oint \vec{u} d V)+\oint \vec{u} \vec{u} \cdot d \vec{A}=-\frac{1}{\rho} \oint p I \cdot d \vec{A}+v \oint \nabla \vec{u} \cdot d \vec{A}
\end{gathered}
$$

where $\vec{u}$ is the velocity vector, $t$ the time, $p$ the static pressure, $\rho$ the blood density, $v$ the kinetic viscosity, $I$ the identity matrix, $d A=d A \vec{n}$ an element of surface area and $\vec{n}$ a vector pointing along the outward normal of the surface $A$. Thus the variation of $\vec{u}$ inside the volume depends only on the normal flux through the surface that defines the volume and the source terms within it.

For transient simulations, the governing equations must be discretized in both space and time. The spatial discretization for the time-dependent equations is identical to the steady-state case. After discretization, the above equations were applied to a discrete control volume $V_{i}$ ( $i$ denotes the volume number):

$$
\begin{gathered}
\sum_{j} \vec{u}_{i, j} \cdot \vec{A}_{i, j}=0 \\
V_{i} \frac{\partial}{\partial t} \vec{u}_{i}+\sum_{J} \vec{u}_{i, j} \vec{u}_{i, j} \cdot \vec{A}_{i, j}=-\frac{1}{\rho} \sum_{j} p_{i, j} I \cdot \vec{A}_{i, j}+v \sum_{j} \nabla \vec{u}_{i, j} \cdot \vec{A}_{i, j}
\end{gathered}
$$


where the sum of the flux-area scalar products refers to all the external sides $j$ of the control volume.

Temporal discretization involves the integration of every term in the differential equations over a time step $\Delta t$. A generic expression for the time evolution of a variable, for the example of a velocity component $u_{x}$ (likewise for the other variables), is given by:

$$
\frac{\partial u_{x}}{\partial t}=F\left(u_{x}\right)
$$

where the function $F$ incorporates any spatial discretization. In the current calculation, the discretization procedure is based on the implicit second-order algorithm to obtain more accurate results. For the variable $u_{x}$, the second-order discretization is given by:

$$
\frac{3 u_{x}^{n+1}-4 u_{x}^{n}+u_{x}^{n-1}}{2 \Delta t}=F\left(u_{x}\right)
$$

where $n+1$ denotes the next time level $(t+\Delta t), n$ denotes the current time level $(t)$; and $n-1$ denotes the previous time level $(t-\Delta t)$.

The advection scheme was second-order upwind, which means that quantities at cell faces are computed using a multidimensional linear reconstruction approach. In this approach, higher-order accuracy is achieved at cell faces through a Taylor series expansion of the cell-centered solution about the cell centroid. Thus when second-order upwinding is selected, the face value for $x$ velocity $\left(u_{x, f}\right)$ is computed using the following expression:

$$
u_{x, f}=u_{x, c}+\nabla u_{x, c} \cdot \Delta \vec{s}
$$

where $u_{x, c}$ and $\nabla u_{x, c}$ are the cell-centered value and its gradient in the upstream cell, and $\Delta \vec{s}$ is the displacement vector from the upstream cell centroid to the face centroid. 
The pressure correction term was calculated using the Pressure-Implicit with Splitting of Operators (PISO) pressure-velocity coupling scheme combined with additional neighbor and skewness corrections. The advantage of using the PISO algorithm is that it ensures newly calculated velocities and corresponding fluxes satisfy the momentum balance after the pressure-correction equation is solved, thus improving the efficiency of the calculation.

To judge the convergence, a "scaled" residual derived from the magnitude of the velocity components (in $x, y$, and $z$ directions) was used in this calculation. For the momentum equations:

$$
R^{m}=\frac{\sum_{\text {cellsi }}\left|\sum_{n b} a_{n b} u_{n b}+b-a_{i} u_{i}\right|}{\sum_{c e l l s i}\left|a_{i} u_{i}\right|} \leq 10^{-4}
$$

where $u_{i}$ is the magnitude of any velocity component at cell $i, u_{n b}$ are the magnitudes of any velocity component at the neighboring cells, $a_{i}$ is the center coefficient and $a_{n b}$ are the influence coefficients for the neighboring cells ( Both of them can be calculated from the discretized governing equations (3.7) and (3.8)), and b is a constant contributed by the boundary conditions. For the continuity equations:

$$
\frac{R_{\text {iteration } N}^{c}}{R_{\text {iteration } 5}^{c}} \leq 10^{-4}
$$

where $R^{c}=\sum_{\text {cellsi }} \mid$ rate of mass creation in cell $i \mid$, and the denominator is the largest absolute value of the continuity residual in the first five iterations.

The computation was carried out on the workstation Origin 3000 (SGI IRIX6.4, 75 $\mathrm{MHz}$ ). Each pulse cycle was divided into 200 400 time steps of size 0.002 0.004s depending on the complexity of the calculation. The typical CPU time per cycle was 
about 18 hours. The iterations were considered converged when both the residual about any velocity component for the momentum equations and the residual about mass creation rate for the continuity equations strictly dropped to $5 \times 10^{-5}$ within each flow cycle. In addition, the mass flow rate on either outlet was monitored during the calculation. Between the second and third cycle, the monitored curve for mass flow rate achieved a steady state and the relative errors in both WSS and its gradient are below $1 \%$. Thus, the computation of two cycles was necessary in order to eradicate any startup effects and achieve repeatability in velocity field and WSS distribution computed in successive cycles.

\subsubsection{Data Analysis}

Post-processing of the results involves calculating cycle-averaged WSS, OSI, SWSSG and TWSSG based on the computation of velocity field, which was accomplished by programming using the Matlab software package (Version 6.5, Release 13, The MathWorks, Inc.). The figures and curves in this thesis were generated using the Tecplot (Version 9.0, Amtec Engineering, Inc.) and Origin (OriginPro 7.5, OriginLab Corporation) software.

The WSS vector is defined by $\vec{\tau}_{w}=\left(\tau_{x}, \tau_{y}, \tau_{z}\right)$, where $\tau_{x}, \tau_{y}, \tau_{z}$ are the WSS components in $x, y, z$ directions, respectively. The cycle-averaged value of the WSS is defined as:

$$
\overline{\left|\vec{\tau}_{w}\right|}=\frac{1}{T} \int_{0}^{T}\left|\vec{\tau}_{w}\right| d t
$$

where $\left|\vec{\tau}_{w}\right|=\sqrt{\tau_{x}{ }^{2}+\tau_{y}{ }^{2}+\tau_{z}{ }^{2}}$ is the magnitude of transient WSS and $T$ is the duration of one flow cycle. The integral was approximated by summing up transient WSSs at 
200 individual time steps equally-distributed over the flow cycle. The WSS was then nondimensionalized or normalized:

$$
\tau_{\text {norm }}=\frac{\overline{\left|\vec{\tau}_{w}\right|}}{\left|\tau_{s}\right|}
$$

where $\tau_{s}=\frac{8 \mu^{2} \mathrm{Re}_{\text {mean }}}{\rho d_{a}{ }^{2}}$, is the Poiseuille-type WSS in the straight native artery at the mean Reynolds number $\operatorname{Re}_{\text {mean }}$.

The OSI provides a good measure of cyclic variations of WSS in three-dimensional space, and the definition takes the form (He and $\mathrm{Ku}, 1996)$ :

$$
\mathrm{OSI}=0.5\left(1-\frac{\left|\int_{0}^{T} \vec{\tau}_{w} d t\right|}{\int_{0}^{T}\left|\vec{\tau}_{w}\right| d t}\right)
$$

The OSI value ranges from 0 to 0.5 depending on the extent of the flow oscillation. The thresholds 0 and 0.5 correspond to completely forward flow and oscillating flow, respectively.

The TWSSG was computed using a second-order central difference approximation method, while the SWSSG was based on the finite element shape functions of quadratic element as described by Either et al. (1998a). Only the results on the centerlines of the floor and hood were presented for interpretation of shear gradient variations over both time and space. On these lines, the normalized SWSSG in the axial direction of the vessel is defined as:

$$
\tau_{S W S S G}=\left|\frac{d \tau_{x} / d x}{\tau_{s} / d_{a}}\right|
$$

where $\tau_{s} / d_{a}$ is the normalization factor. The normalized TWSSG is defined as: 


$$
\tau_{\text {TWSSG }}=\left|\frac{d \tau_{x} / d t}{\left(d \tau_{x} / d t\right)_{S}}\right|
$$

where $\left(d \tau_{x} / d t\right)_{s}$ is the maximum TWSSG for fully developed flow in a long straight artery using the same flow waveform.

\subsection{COMPARISON WITH LITERATURES}

The numerical code was compared with the magnetic resonance imaging (MRI) experimental study conducted by Sherwin et al. (2000) and numerical pulsatile flow study by Ethier et al. (1998b), using the same model geometries and identical flow conditions. For the comparison with the MRI results, a constant flow rate of $5.512 \mathrm{ml} / \mathrm{s}$ was applied corresponding to a Reynolds number of $\mathrm{Re}=250$ based on the internal diameter of the pipe $(8 \mathrm{~mm})$ and the kinetic viscosity of the fluid $\left(3.416 \times 10^{-6} \mathrm{~m}^{2} / \mathrm{s}\right)$. For the pulsatile flow comparison, the physiological LAD waveform was used. The mean Reynolds number and Womersley number based on the typical LAD diameter of $3.3 \mathrm{~mm}$ were 230 and 2.4 , respectively.

Figure 3.6 shows the comparisons of the axial (X direction) velocity component at three locations of the host artery (i.e. at $0.25 d_{a}, 2 d_{a}$ and $5 d_{a}$ distal to the toe) between the numerical and MRI results. All data are normalized by the mean velocity. Good agreement has been observed in comparing the flow patterns at corresponding locations. Clearly, the parabolic axial flow pattern of the Hagen-Poiseuille flow at $0.25 d_{a}$ slightly skewed toward the artery floor. This is due to the existence of a sharp bend where the flow is being continually driven across the host vessel. An even more skewed Dean-type flow is observed at $2 d_{a}$ distal to the toe for both cases. At $5 d_{a}$ distal to the toe, the crescent nature of the flow is still evident, but the viscous effects have started to play a greater role as the profile diffuses back to its steady-state parabolic profile. A 
quantitative comparison in velocity plots along the $\mathrm{Z}$ direction yields to the maximum relative error of about $4.6 \%$.

Figure 3.7 compares the unsteady shear variations along the floor of the host vessel over both space and time. For the more challenging case of unsteady flows, the two studies are still in good qualitative agreement except some minor differences that are not expected to be significant in predicting the disease location and severity. Also, very good agreement is found in the localization of the stagnation point as well as the WSS magnitude.

In conclusion, the comparison with the published MRI and numerical studies has suggested that that the current numerical code produces meaningful results and is reliable for simulating arterial bypass flows. 
(Uiū)

\subsection{0 .400 .600 .801 .001 .201 .401 .601 .80}
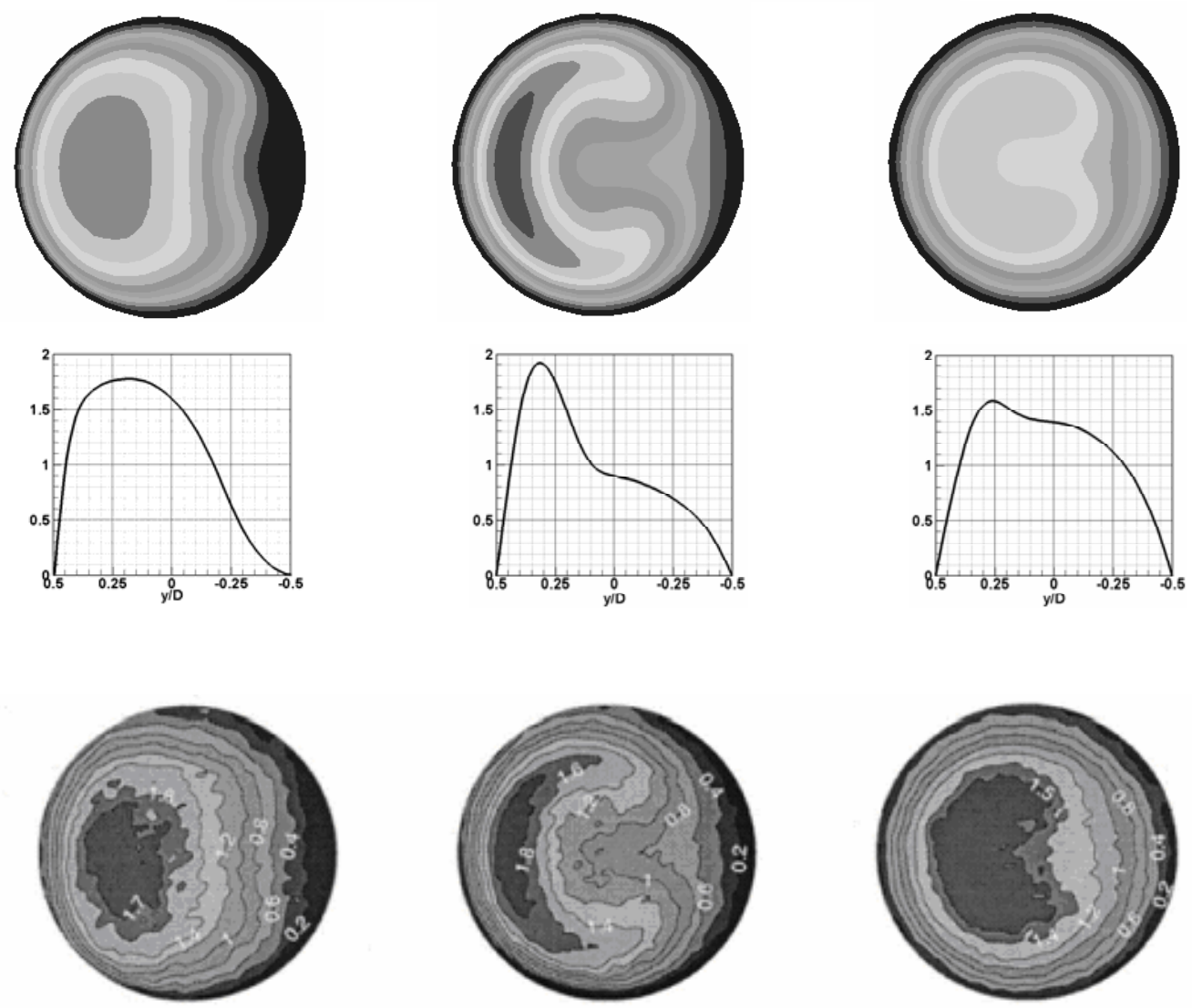

(a)

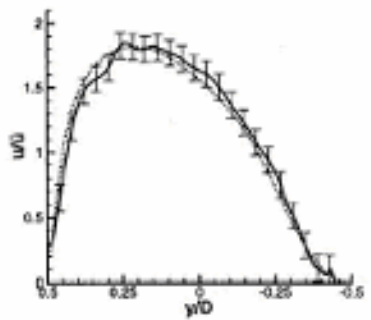

(b)

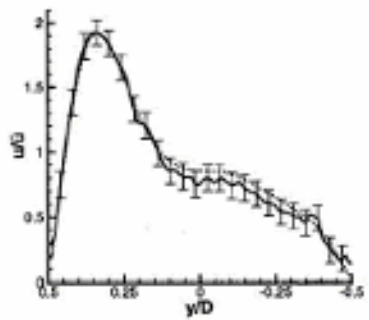

(c)

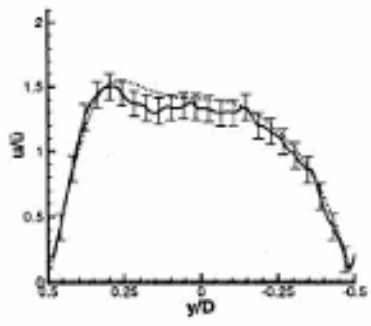

Fig. 3.6 Comparisons of the axial velocity component at (a) $0.25 d_{a}$, (b) $2 d_{a}$ and (c) 5 $d_{a}$ distal to the toe between the numerical results from the present study (top) and the MRI results from Sherwin et al. (2000) (bottom). Also shown are velocity profiles extracted along the constant z centerline. Here, $d_{a}=8 \mathrm{~mm}$, is the host vessel diameter. 

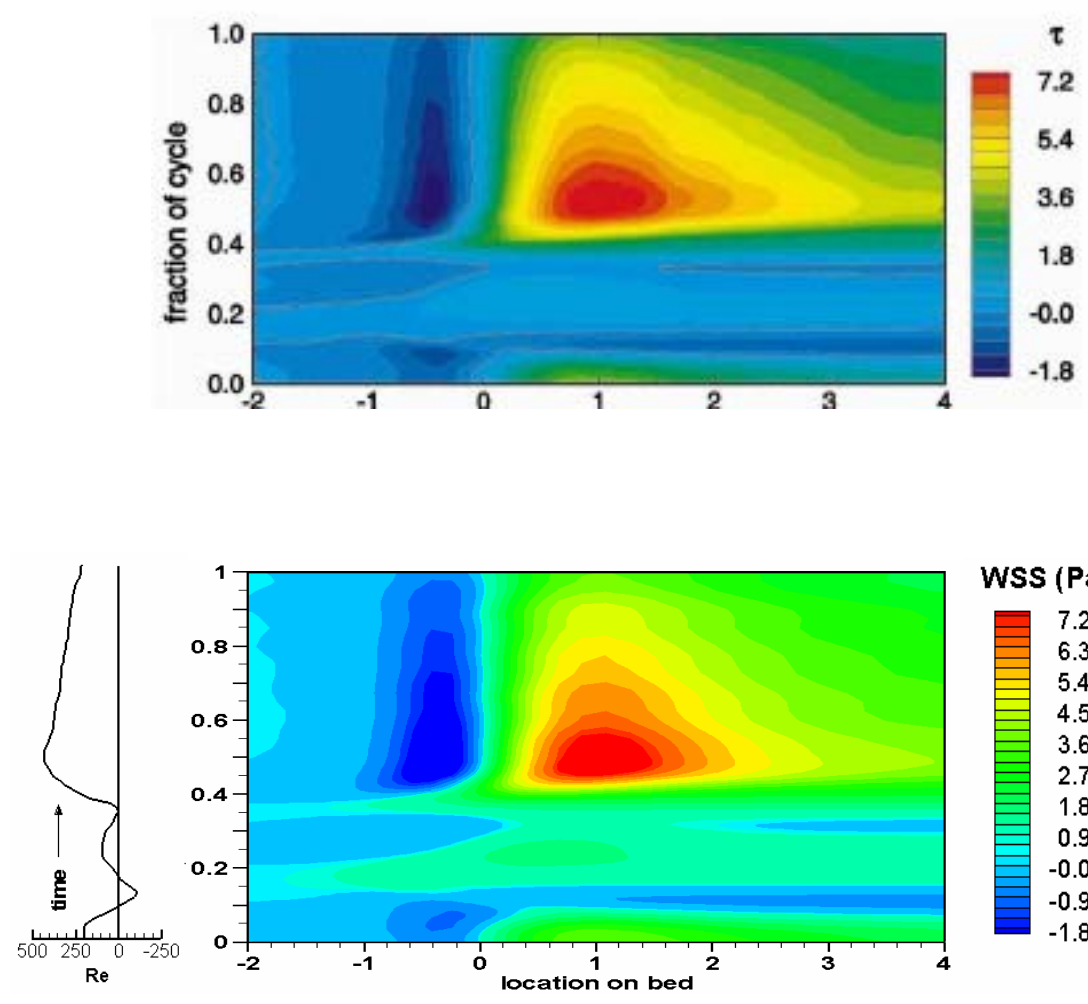

WSS (Pa)

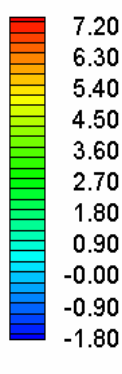

Fig. 3.7 Numerically computed axial WSS along the bed against time using the LAD waveform from the present study (top) and the study of Either et al. (1998) (bottom). 


\section{CHAPTER 4}

\section{TEST ON DISEASE-TRIGGERING MECHANISMS IN CORONARY ANASTOMOSIS}

\subsection{INTRODUCTION}

As discussed in Chapter 2, recurrent atherosclerosis and intimal hyperplasia threaten the graft viability (Bourassa et al., 1984; FitzGibbon et al., 1996). Atherosclerosis is triggered by patient-related risk factors, and the pathogenesis involves accumulation of lipid-filled macrophages and proliferation of smooth muscle cells, whilst intimal hyperplasia is mainly caused by scar tissue formed after the bypass surgery followed by smooth muscle cell proliferation. The two diseases share some similarities in appearance and progression process, and they may also occur concurrently.

Chronic intimal thickening (IT) of the vessel wall caused by atherosclerosis and intimal hyperplasia is a common consequence for all vascular systems, characterizing reproducible sites in natural or reconstructed bifurcations where disturbed local hemodynamics has been demonstrated (Ku et al., 1985; Bassiouny et al., 1992; Keynton et al., 2001). Although hemodynamics is believed to play an important role, it has not been completely elucidated as to what aspects of hemodynamics are most influential upon the disease process and how the influence is exerted. As both types of IT are degenerative responses of the vessel wall, several hemodynamic wall parameters, including WSS, OSI, SWSSG and TWSSG, have been postulated to quantify the influence with respect to different aspects of hemodynamics. There has been much effort trying to link these parameters with IT development in vivo. For example, Gibson 
et al. (1993) via a serial quantitative coronary angiography technique revealed that local rates of atherosclerosis progression in patients with coronary artery disease correlate inversely with the magnitude of WSS in the condition of controlled systemic risk factors. For polytetrafluoroethylene (PTFE) tapered grafts in canine models, an inverse, nonlinear relationship between the amount of shear stress and neointimal thickness was established by Ku and Zhu (1993). Studies incorporating pulsatility of physiological flow found that not only low absolute magnitude of WSS but also its directional fluctuations are atherogenic (Ku et al., 1985; Bassiouny et al., 1992; Keynton, et al., 2001), and the OSI can be used to measure this combined effect. Evidences in high WSS theory were generally provided in studies on smaller animals. In cholesterol-fed (Cornhill and Roach, 1976) and low-density lipoprotein receptor-deficient (Rosenfeld et al., 1987) rabbits, lesions often develop at regions downstream of the bifurcations in larger arteries where the highest WSS is detected. Furthermore, Lei et al. (1995) using the aorto-celiac junction of rabbits as a representative atherosclerotic model showed that the regions with high SWSSG match the sites susceptible to atherosclerotic lesions.

However, the previous correlation studies have been performed either for native artery bifurcations or for bypass anastomoses in peripheral regions or animals. There has been scarcity of such work dedicated to the human coronary artery anastomoses. It has been recognized that the localization and severity of lesions in coronary anastomoses differ from those seen in peripheral systems (Butany et al., 1998; Ojha et al., 2000). This phenomenon can be attributed to the fact that autologous veins or arteries are usually preferred in CABG as graft conduits, while synthetic PTFE tubes are commonly used in peripheral artery grafting (Reardon et al., 1997). Also, fundamental differences in biological environment of the vessel wall exist between different species, different types of vessels, and even different locations, which may constitute part of the reason. 
Therefore, certain hemodynamic factors having little impact on tissues in peripheral anastomotic systems may affect significantly on those in the coronary region. As such, the dominating factors causing IT in human coronary anastomoses are expected to be different. The goal of the study in this chapter is to establish a causative relationship between hemodynamic factors and IT in coronary anastomoses, by correlating the widely-hypothesized hemodynamic factors with statistical histological data of IT in human coronary anastomoses reported in the literature (Butany et al., 1998; Ojha et al., 2000). Understanding this relationship would help define proper indicators for predicting disease-prone sites and assessing hemodynamic performance of coronary anastomoses as intended in the following chapters.

\subsection{NUMERICAL MODEL AND FLOW CONDITIONS}

A representative human end-to-side coronary anastomotic geometry was constructed. The primary geometric features of the numerical model, i.e. diameters of the graft $(D=$ $5.3 \mathrm{~mm}$ ) and host artery $\left(d_{a}=2.2 \mathrm{~mm}\right)$ and a steep local curvature on the hood, conformed to a human coronary anastomosis of saphenous vein (SV) to left anterior descending artery (LAD) as described in Leask (2002). Patient-specific small-scale surface features have been shown to be of secondary importance (Moore et al., 1999) and thus neglected in this study. Figure 4.1 shows the model geometry with relevant terminology. Considering the symmetry in the model, only half of the domain was simulated. Based on a mesh-independence study, a mesh of 128,936 hexahedral cells represents a trade-off between accuracy of results and computational overheads.

Fully-developed pulsatile velocity profile and zero surface traction were applied at the inflow and outflow boundaries, respectively. The proximal-distal outflow ratio was specified to be 20:80. Two waveforms representative of flows in the LAD (Kajiya, 
1986) and SV graft (Galjee et al., 1996) were employed (Fig. 4.2), both giving a mean Reynolds number $\left(\operatorname{Re}_{\text {mean }}=4 \rho Q_{\text {meam }} / \pi \mu D\right.$; where $Q_{\text {mean }}$ is the mean volume flow rate at the inflow boundary, $\mu$ the dynamic viscosity, and $\rho$ the density) of 110 and a Womersley unsteadiness parameter ( $W o=\frac{D}{2} \sqrt{\frac{\omega \rho}{\mu}}$; where $\omega$ is the fundamental frequency) of 2 based on the diameter of the inlet vessel (Leask, 2002). The inlet and outlet segments were extended about 12 and $20 d_{a}$, respectively, from the center of the anastomotic region so as to avoid perturbations on the anastomotic flow field. Non-slip condition was applied at all walls.

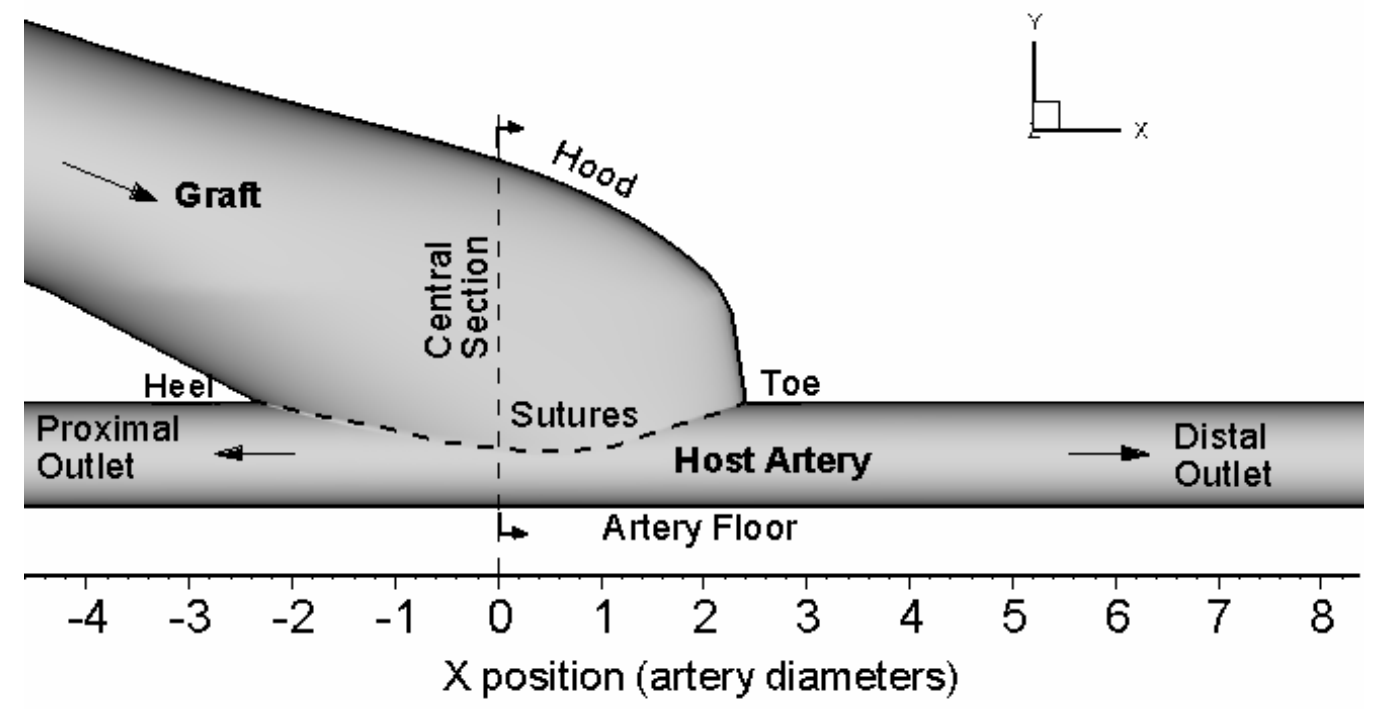

Fig. 4.1 The numerical model with relevant terminology. 


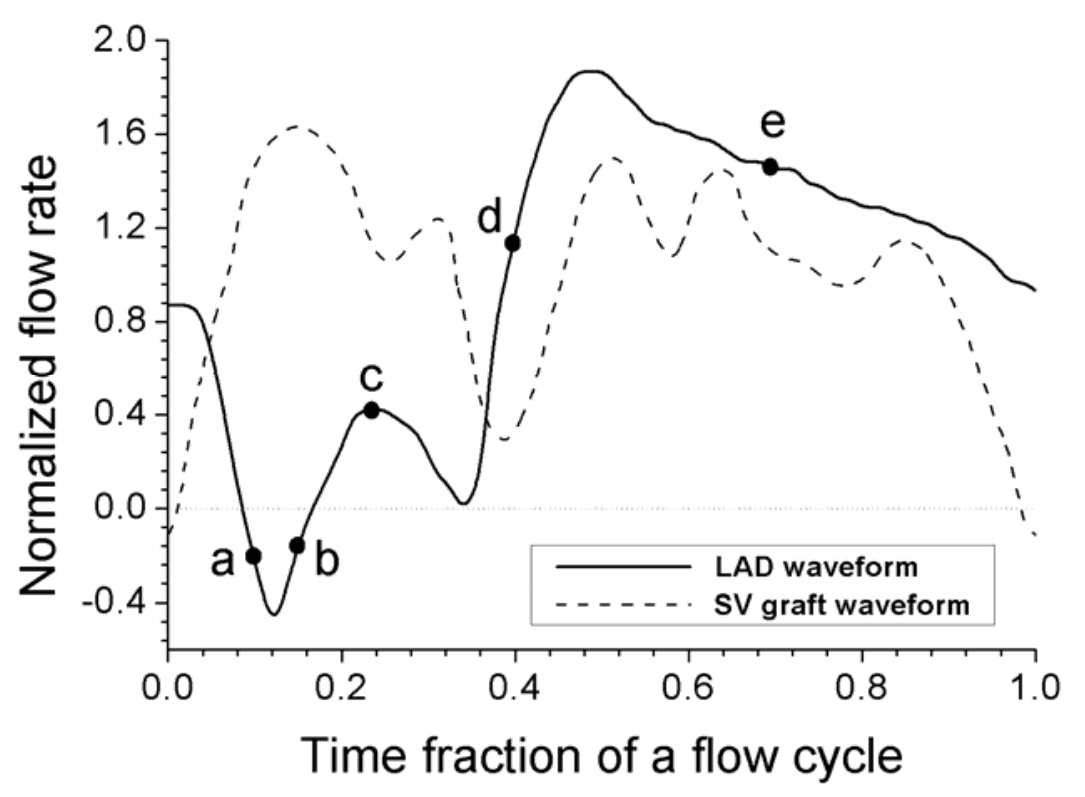

Fig. 4.2 Waveforms simulating flows in arterial and venous grafts. Both were scaled to give a mean Reynolds number of 110 and a Womersley number of 2 based on the inlet

vessel diameter of $5.3 \mathrm{~mm}$. Labels indicate time steps at which flow patterns are presented, including early negative flow (a), late negative flow (b), mid systole (c), diastolic acceleration (d), and diastolic deceleration (e).

\subsection{RESULTS}

A small portion of negative phase in the LAD flow waveform, as a result of cardiac muscle contraction, produces significant flow instabilities during early systole. The SV waveform, however, has a much smaller negative portion and more equally distributed flow between systole and diastole, giving rise to less variation in transient flow patterns. Figure 4.3 shows main flow characteristics occurring during the flow cycle of the LAD waveform. Shortly after the flow has transited from positive to negative phase, the velocity profiles across the host artery become retrograde and exhibit a M-shape with smaller flow in the center and two peaks near the upper and lower walls (Fig. 4.3a). The low-momentum flow near the wall of the graft is easily driven backward when suddenly subject to a negative pressure gradient, whereas the high-momentum flow in the mainstream does not yet become retrograde until it reaches the distal end of the graft. 
Consequently, a region of low-momentum flow recirculation is formed in the proximal anastomotic region as illustrated by the streamtraces. Extremely low flow is also observed along the proximal part of the floor segment opposite to the graft orifice. As the negative flow proceeds to a later stage, the flow profile across the distal artery changes to a quasi-parabolic shape (Fig. 4.3b). The low flow area on the floor is extended to cover almost the entire floor segment opposite to the graft orifice. Flow separation is demonstrated along the hood, resulting in an extensive area in the vicinity filled with low and stagnant flow. At mid systole when the flow returns to its primary direction, the flow separation along the hood is cleared but the flow velocity remains low (Fig. 4.3c). This low flow disappears during diastolic acceleration but is promoted again in the deceleration phase and persists over the remainder of the cycle (Fig. 4.3d,e).

The WSS distribution patterns for the LAD and SV waveforms are identical (Fig. 4.4). The lowest WSS characterizes an extensive area on the hood of the graft just in the vicinity of the central section. The second lowest WSS covers the majority of the graft body as well as the native artery segment adjoining the graft orifice. The level of WSS is generally lower in the graft and proximal artery segment than in the distal artery segment. The highest values are delivered to the toe and the lateral wall of the artery across the toe.

The regions with high OSI closely resemble those with low cycle-averaged WSS (Fig. 4.5). The area of high OSI is most prominent on the hood around the central section. Elevated OSI also resides on the upper wall of the distal artery immediately distal to the toe, the graft inner wall across the hood, as well as the artery floor near the central section. The LAD waveform exhibits a slightly higher OSI level, specifically on the 
graft body, as compared to the SV waveform. The entire proximal and distal artery segments show low OSI with magnitudes below 0.05 .

Figure 4.6 shows variations of SWSSG on the artery floor over both space and time for the LAD and SV waveforms. For a region with uniform flow, the magnitude of SWSSG would be zero, so any value of SWSSG denotes disturbed hemodynamics. For both waveforms, elevated SWSSG covers the entire floor segment opposite the graft orifice, with the maximum normalized value of 5.52 6.26 delivered to the position about $1 d_{a}$ distal to the central section. Axially from the anastomotic region, the magnitude of SWSSG approaches zero. The gradient is flow rate dependent, e.g. is higher in the diastole than in the systole for the LAD waveform and reaches the maximum at the peak flow rate (at about $1 / 2$ of the flow cycle). For the SV waveform, elevated values are distributed evenly in the systolic and diastolic phases. Although the transient SWSSG over the flow cycle for the two waveforms are different, the distributions of cycleaveraged SWSSG share similar patterns. Figure 4.7 illustrates the spatial variation of cycle-averaged SWSSG for the LAD waveform in contrast to the variation of OSI. Note that elevated SWSSG and elevated OSI are located very close to each other around the central section on the artery floor.

For both waveforms, elevated TWSSG occurs on the floor of the distal anastomotic region as well as the entire floor of the distal artery segment, leaving the area around the central section relatively spared (Fig. 4.8). The highest magnitude of TWSSG (1.83 2.01) is found at the region just distal to the toe, more than $2 d_{a}$ away from the central section. The temporal gradient is dependent on the slope of the waveform, e.g. the gradient reaches the maximum at about $2 / 5$ of the flow cycle in the LAD case. 
Figure 4.9 shows that the entire segment on the hood upstream of the position $\mathrm{X}=2 d_{a}$ is devoid of both elevated SWSSG and TWSSG. Extremely high SWSSG is observed at the toe, whilst high TWSSG is extended downstream from the toe to cover the entire distal artery segment.
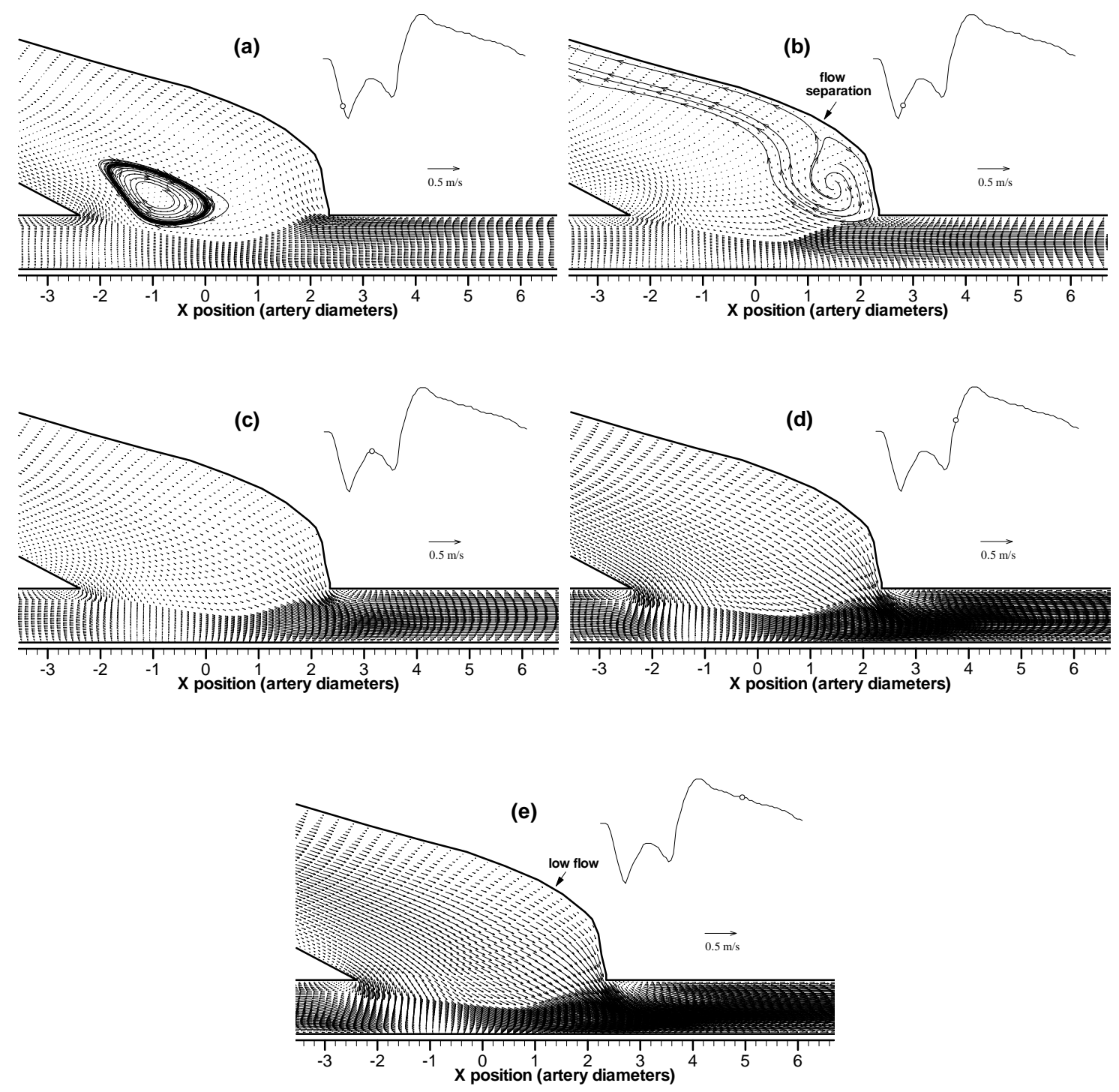

Fig. 4.3 Velocity vector distributions on the symmetry plane: (a) early negative flow, (b) late negative flow, (c) mid systole, (d) diastolic acceleration, and (e) diastolic deceleration. Streamtraces are used to enhance the data presentation. 

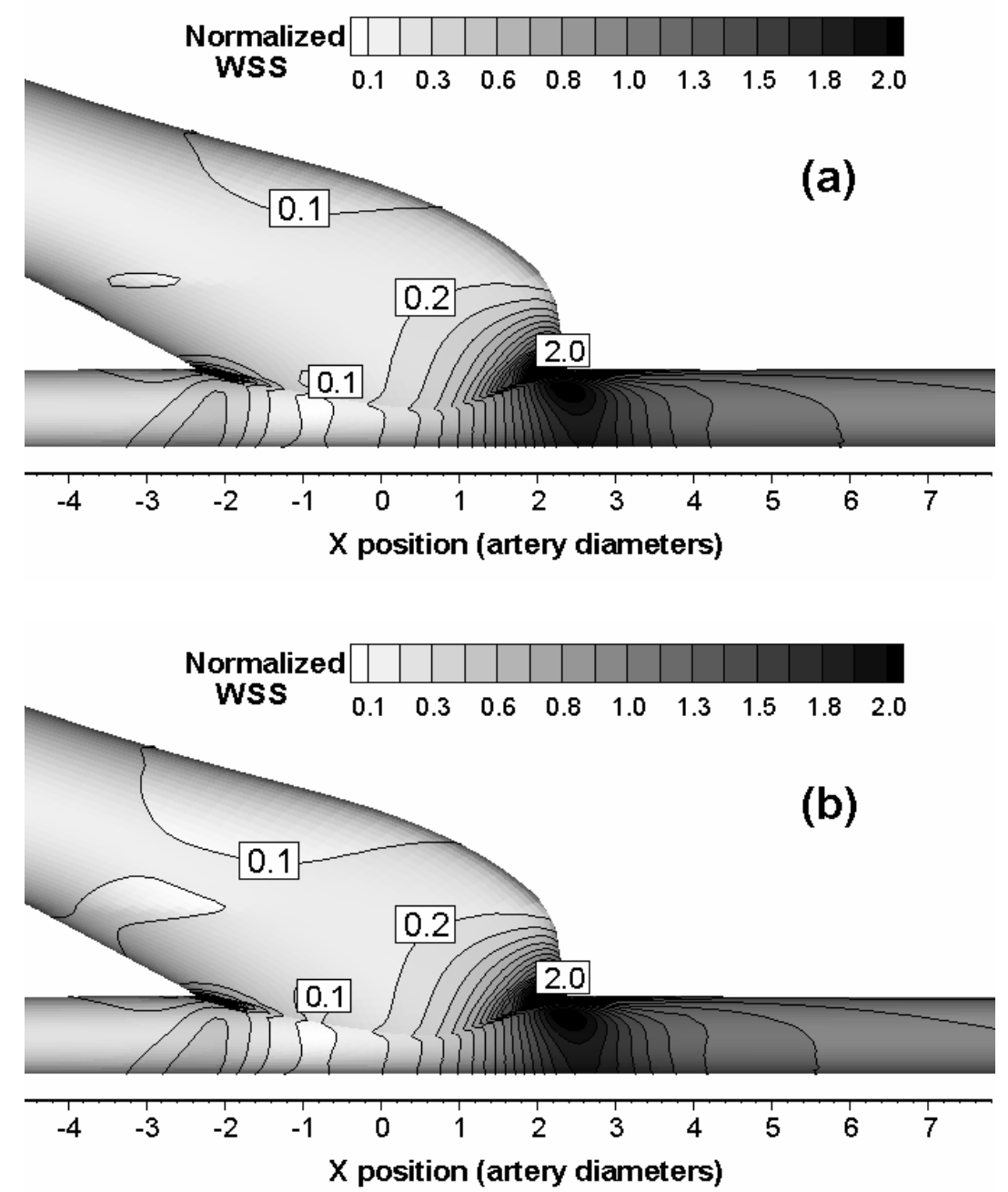

Fig. 4.4 Contour plots of cycle-averaged WSS: (a) LAD waveform, and (b) SV flow waveform. 

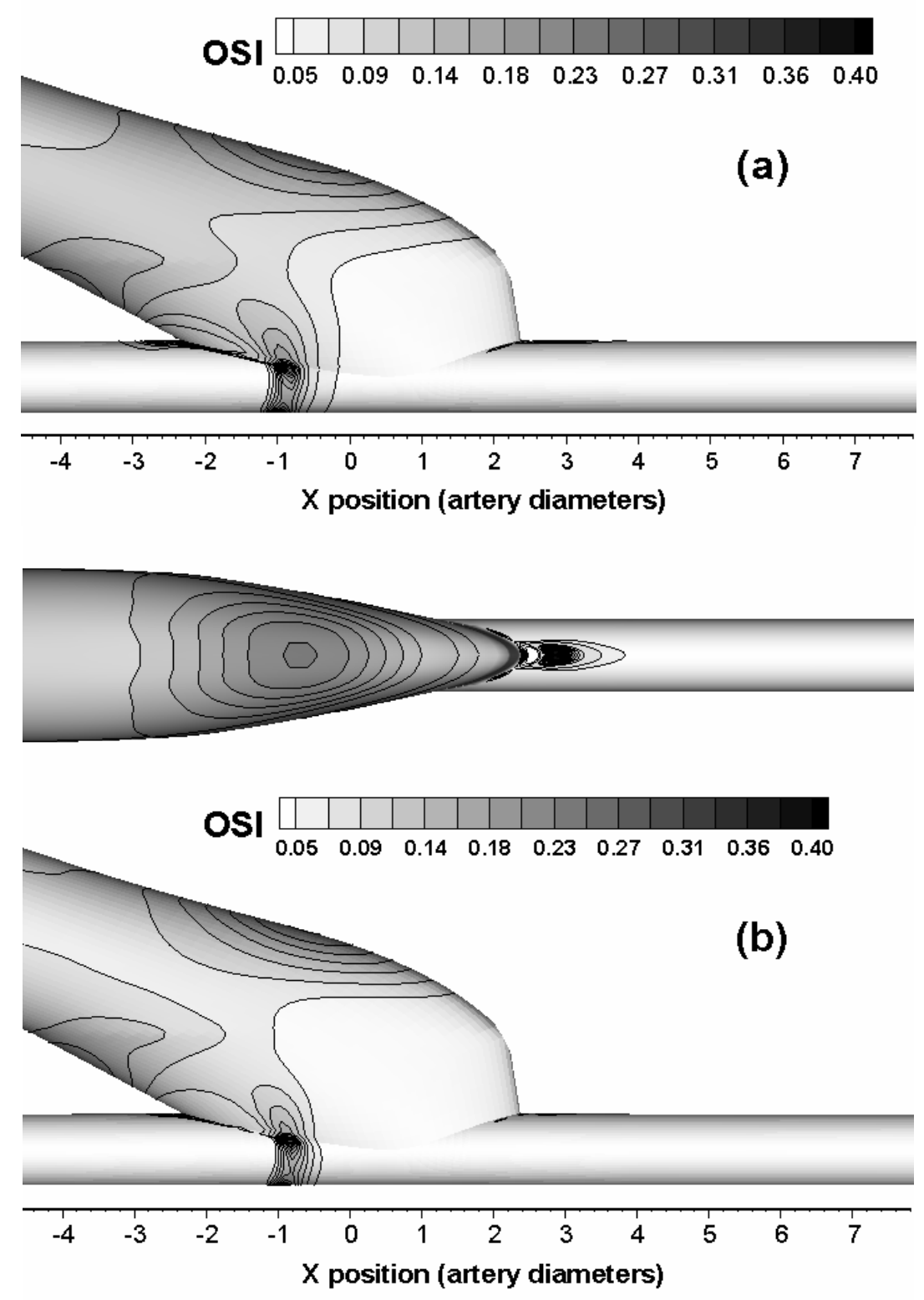

Fig. 4.5 Contour plots of OSI: (a) LAD waveform, and (b) SV flow waveform. 
(a)
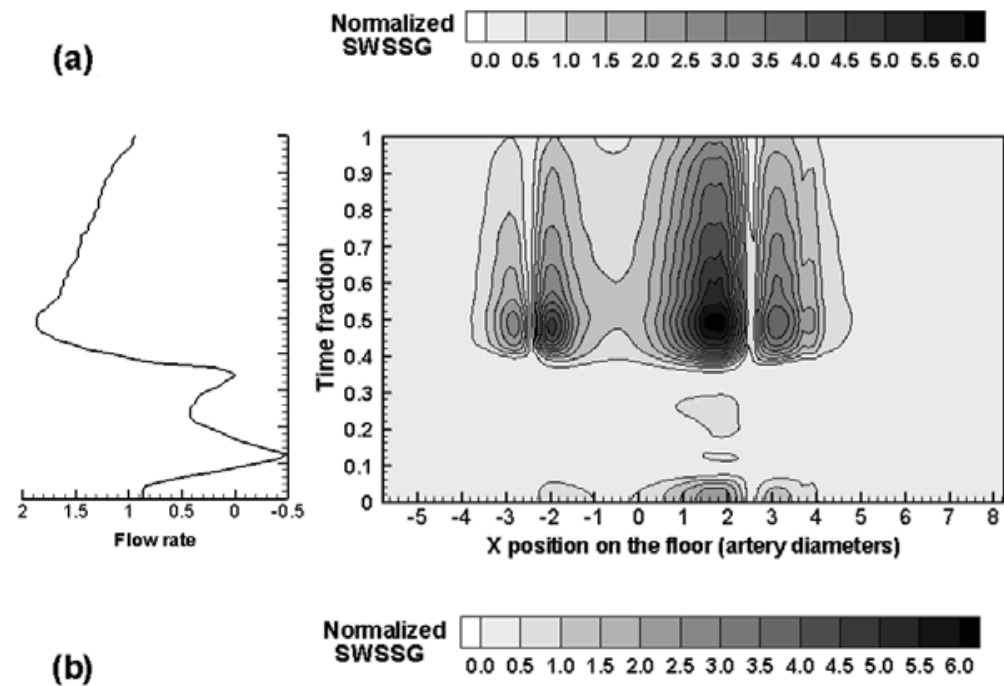

(b)

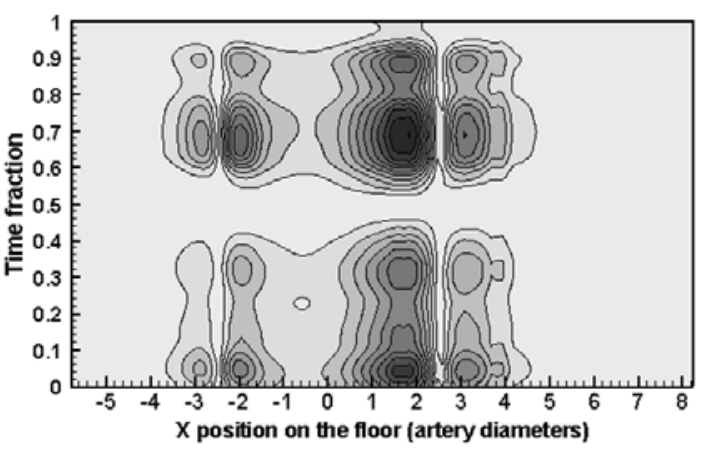

Fig. 4.6 SWSSG variations along the artery floor over both space and time: (a) LAD waveform, and (b) SV flow waveform.

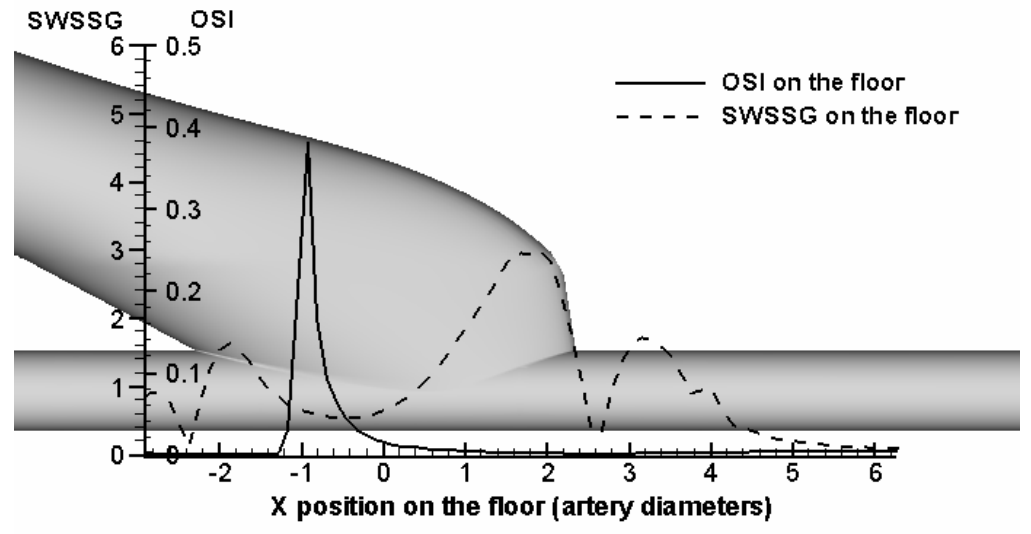

Fig. 4.7 Variations of cycle-averaged SWSSG and OSI along the artery floor for the LAD waveform. 
(a)
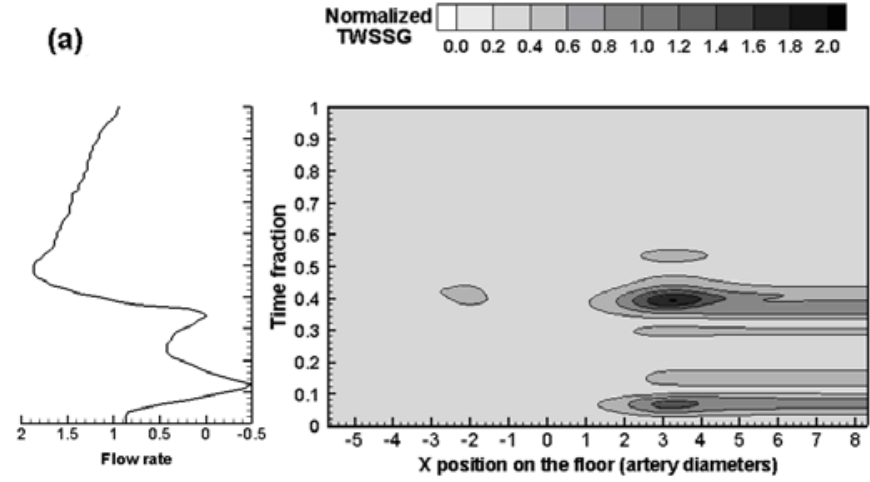

(b)
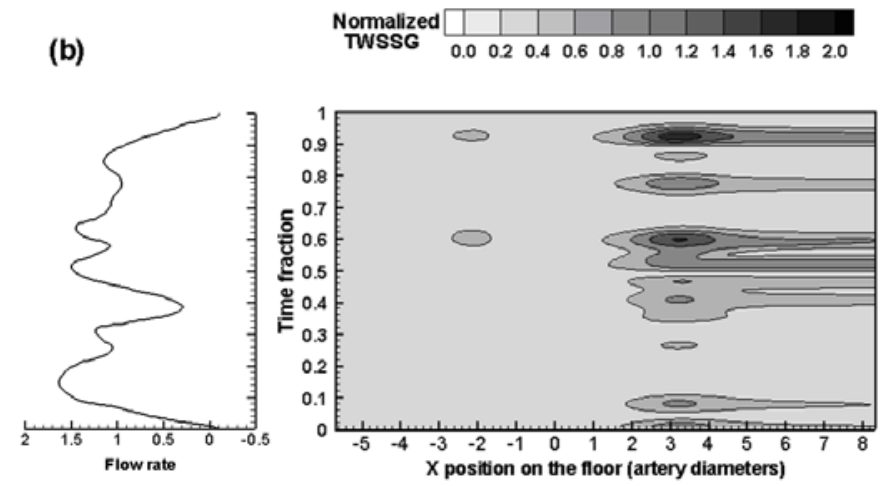

Fig. 4.8 TWSSG variations along the artery floor over both space and time: (a) LAD waveform, and (b) SV flow waveform.

(a)
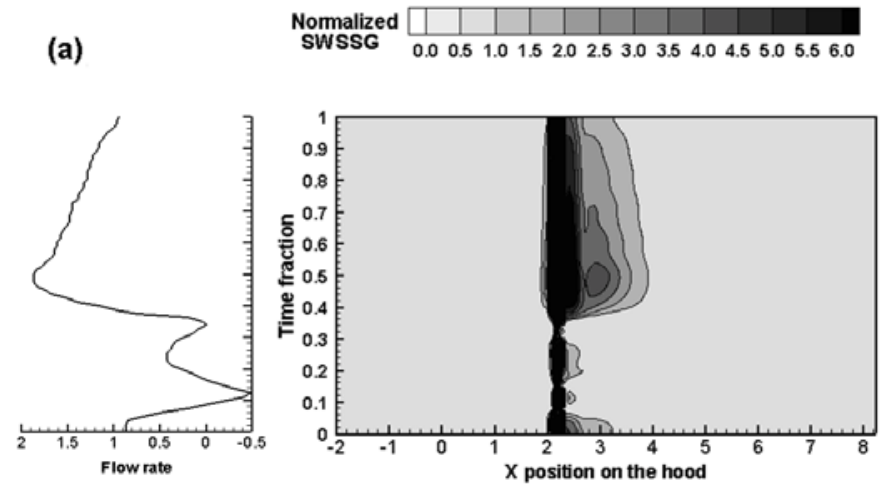

(b)
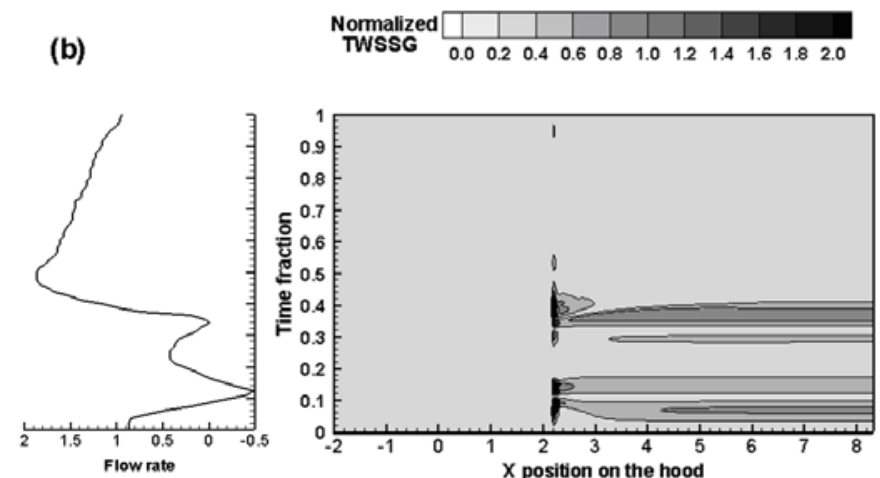

Fig. 4.9 Variations of (a) SWSSG and (b) TWSSG along the hood and the upper wall of the distal artery over both space and time for the LAD waveform. 


\subsection{DISCUSSION}

Each of the hemodynamic parameters considered in this study, i.e. WSS, OSI, SWSSG and TWSSG, may exert, in its unique way, critical and diverse influence upon functioning of the endothelium, endothelial gene expression and phenotype switching, intimal and smooth muscle cell proliferations, transport of macromolecules in blood (e.g. LDL), as well as monocyte (platelets, leukocytes) accumulation. For example, low WSS is known to adversely affect elongation and orientation of the endothelial cells, resulting in an aggressive and proliferative phenotype (Davies, 1995). Greater intimal and smooth muscle cell proliferative responses could also be promoted in low WSS or high OSI regions by inducing the expression of the platelet-derived growth factor (PDGF-A) and messenger RNA (Kraiss et al. 1996). Low and oscillatory shear or high OSI may also increase surface concentration of LDL and promote leukocyte binding into the vessel wall (Deng et al., 1995; Walpola et al., 1993). High SWSSG is suggested to cause severe local deformation and trauma to the apical cell membrane and associated structures (DePaola et al., 1992; Truskey et al., 1995; Satcher et al., 1991), therefore breaking down consistent signaling and communication between cells and resulting in elevated wall permeability to macromolecules which enter the vessel wall by transmural filtration (Lei et al., 1995; Buchanan et al., 1998). High TWSSG is suggested to disrupt the atheroprotective molecular pathways, inducing the production of proinflammatory mediators, impaired flow regulated vasorelaxation and impaired functions of the antiapoptotic, antiproliferative (relevant to vascular SMC) and antioxidative (relevant to LDL) of the endothelium (Ziegler et al., 1998). Cumulatively, the effects of the hemodynamic factors on the initiation and progression of the vascular diseases are multifaceted and there exist diverse mechanisms. While the general mechanisms are common to all systems in the vascular tree, the dominating ones may 
vary over locations and systems. The present study therefore seeks to determine the dominating hemodynamic factors associated with IT in distal coronary anastomoses.

In this study, the hemodynamic parameters were correlated with quantitative information on IT distributions at anatomical landmarks such as the suture sites, hood, graft body and floor reported in the histological studies of Butany et al. (1998) and Ojha et al. (2000). Figure 4.10 summarizes and compares the histological data of late venous and arterial grafts (> 2 months) (Ojha et al., 2000). The data revealed that IT develops around the suture line, the hood, and the floor of the central section, as well as throughout the entire graft body, and the venous grafts shows the greatest IT on the hood, even greater than that at the suture sites. It should be recognized that compliance mismatch, suture-line stress concentration and local injury may contribute to IT at the suture sites (Ballyk et al., 1998; Hofer et al., 1996; Weston et al., 1992), and the graft material characteristics may also play a role, e.g. intimal hyperplasia that grows along synthetic and venous grafts tends to be thicker than along arterial grafts (Sisto et al., 1989). The histological observations indicate that the greatest IT on the hood is neither a continuation from the thickening at the suture sites nor solely related to the graft material (Butany et al., 1998; Ojha et al., 2000); there must be hemodynamic forces that exert strong influence on this region. The present numerical results show that both SWSSG and TWSSG on the hood are nearly zero and do not seem to be the cause. Low WSS and high OSI, which exhibit the severest level on the hood exactly around the central section, are more likely to be involved. In addition to the hood, the entire wall of the graft is covered by low WSS and elevated OSI, which may explain the diffuse IT developed in the graft body reported by Butany et al. (1998) and Ojha et al. (2000). Ojha et al. (2000) revealed that IT in the venous graft is on average 10 times greater than that in the arterial graft, and the thickening is progressive in the former but not in 
the latter. This observation suggests that venous grafts may intrinsically have a higher tendency towards IT than arterial grafts, which reconfirms the earlier findings (Sisto et $a l .$, 1989). There is evidence that the morphological sequence of thickening process in venous grafts is different from that in arterial grafts. Endothelial cells in healed venous grafts do not prevent the development of occlusive IT as arterial endothelium does; that is, venous endothelial cells fail to serve as a competent blood flow transducer or protector in arterial conditions (Berger et al., 1972). Vein grafting is followed by a rapid deposition of molecules such as platelets and leucocytes, which secrete cytokines to stimulate vascular smooth muscle cell proliferation and migration (Golledge, 1997). These events are favored by low WSS and high OSI (Kraiss et al., 1996), consistent with our findings and argument that low WSS and high OSI induce IT in the graft body.

The present study shows that low WSS and high OSI are also found on the artery floor near the central section, adjacent to which is a long segment characterized by elevated SWSSG. This finding suggests that IT on the floor of the central section may be caused by a combination of multiple factors including low WSS, high OSI and high SWSSG. It is likely that when high OSI and high SWSSG colocalize, elevated concentration of macromolecules in blood induced by high OSI would readily enter the subendothelium through the intercellular junction due to enhanced endothelial permeability caused by high SWSSG, leading to focal IT. If one considers other factors such as high WSS or high TWSSG, there does not appear to be much correlation with IT at the examined central section. Besides, the TWSSG is strongly waveform-dependent, and the elevated values last for only about $1 / 3$ of a flow cycle for the LAD waveform.

Comparing the two sets of data in Fig. 4.10, it is important to note that intimal thickness at all sites of the late venous grafts, including the floor, was more severe than in the 
arterial grafts. IT on the floor is not considered to be directly related to the graft type, so the venous and arterial grafts should have a comparable thickness on the floor given identical flow conditions and vessel geometry. This is indeed the case for the early samples of 12 days to 2 months (Butany et al., 1998; Ojha et al., 2000). Greater floor thickening in the late venous grafts can be an indirect result of early-developed IT on the hood, suture sites and graft body, causing unfavorable alteration to the anastomotic geometry which would worsen local hemodynamics and favor IT development on the floor.

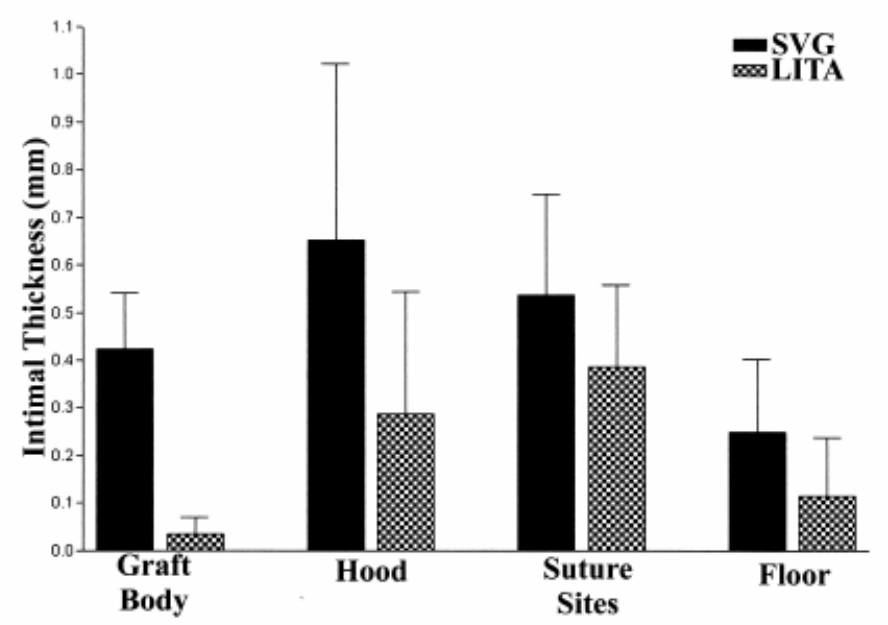

Fig. 4.10 Statistical data of IT in the venous and arterial grafts (from Ojha et al., 2000, with permission).

The flow in the LAD conditions shows greater instabilities as a result of a negative flow during systole than the SV counterpart. However, the overall and cycle-averaged distributions of wall parameters are identical for both waveforms. Myers et al. (2001) compared the effect of a sinusoidal and a physiological right coronary flow waveform on flow and WSS in the right coronary artery and found that the waveform shape is not an important factor in cycle-averaged parameters as long as the peak, mean, and reverse-flow interval for the waveforms are similar, which concurs with the findings of the present study. One may therefore speculate that the use of other patient-specific 
coronary waveforms would yield the same conclusions regarding the cause of IT. It can also be suggested that the cycle-averaged wall parameters represent an averaged flow field and can be used to efficiently evaluate hemodynamic performance of a bypass anastomosis in a quantitative manner, but they cannot describe detailed flow characteristics such as formation of vortex and flow separation. Detailed transient flow patterns can reflect directly how the bypass geometry and input waveform affect the behavior of blood flow. This thesis, therefore, will consider both transient flow patterns and wall parameters in predicting lesion prone sites and measuring bypass performance.

Significant IT on the hood has rarely been reported for peripheral bypasses, in which the suture sites and the floor region are most notorious (Bassiouny et al., 1992; Keynton et al., 2001). Apart from different materials used for the coronary and peripheral grafts, the preponderance of hood thickening in SV grafts reported by Butany et al. (1998) may lie partly in the sharp local curvature on the hood as well as the significant size discrepancy between the graft and artery, causing the flow and WSS on the hood and throughout the graft to be considerably low as demonstrated in the present study. The peripheral anastomoses, in comparison, usually have less degree of diameter mismatch between the graft and artery and a higher mean Reynolds number, which would increase blood flow and eliminate low WSS in the graft and thus reduce tendency towards IT development. High graft flow, on the other hand, would exert strong impinging force on the artery floor, resulting in extremely high SWSSG associated with enhanced endothelial permeability making the floor of the peripheral anastomoses more vulnerable to IT. It can therefore be implied that the significant IT in the venous grafts of coronary anastomoses is likely to be suppressed by reducing graft-artery diameter mismatch and eliminating the steep local curvature on the hood. 
In conclusion, IT processes at different sites of distal coronary anastomoses are dominated by different hemodynamic factors. IT on the hood and graft body is more likely to be associated with low WSS and high OSI, and the venous graft favors its progression; whilst both high OSI and high SWSSG are responsible for the IT on the artery floor. However, there are several limitations in this study. Firstly, the histological data has been presented in anatomical landmarks of the central cross-section of the anastomosis. Disease distributions in longitudinal sections, which would offer more information for correlation with our hemodynamic study, are not available. Secondly, only one anastomotic geometry has been investigated due to lack of detailed sample dimensions. Establishing a precise relationship between hemodynamic factors and IT would entail a great number of realistic geometries and corresponding pathological information. In addition, the flow conditions used in our study such as the flow division ratio and flow rate may not represent the actual situation occurring in the grafts. It is believed that a more statistical correlation could be made if these data are available. 


\section{CHAPTER 5}

\section{GEOMETRIC INVESTIGATIONS AND OPTIMAL DESIGN OF DISTAL CORONARY ANASTOMOSIS}

\subsection{INTRODUCTION}

Geometric factors profoundly alter local hemodynamics in natural bifurcations or reconstructed vascular anastomoses, particularly at the distal part (Friedman et al., 1989; Crawshaw et al., 1980). This close relationship has stimulated much work, mostly in peripheral regions, to optimize anastomotic geometries (e.g. graft-artery diameter ratio, graft-artery angle, and toe and heel transitional curvatures) so as to improve hemodynamics and find ways to inhibit the occurrence of restenosis (Lei et al., 1997; Keynton et al., 1999; Hughes and How, 1996; Moore et al., 1999; Jackson et al., 2001; White et al., 1993). For example, Lei et al. (1997) in their numerical study has suggested that the graft-artery diameter ratio affects the distribution and magnitude of spatial shear gradient. Most work concerning graft-artery angle has been conducted in steady-state or simplified sinusoidal flow conditions, and has found that the angle of an anastomosis has potent influences upon extent of flow skewing and separation at the entrance of the host artery and thus distributions of shear stress and its spatial gradient on the artery wall (Keynton et al., 1991; Fei et al., 1994; Hughes and How, 1996). In addition, for infragenicular revascularizations, there are clinical evidences that interposition vein (Miller) cuffs have obviously increased graft viability (Miller et al., 1984; Stonebridge et al., 1995; Morris et al., 1993). The gains are thought to be mainly a result of improved hemodynamics via altered anastomotic geometry, causing redistribution of distal anastomotic IH away from critical areas (How et al., 2000; 
Wijesinghe et al., 1999; Harris and How, 1999; Da Silva et al., 1997). All these studies underscore the importance of geometric factors and their specific roles in regulating anastomotic hemodynamics.

Thus far, however, such work on geometric optimization dedicated for coronary anastomoses has either not been conducted or has been limited to steady flow conditions (Song et al., 2000), and there has long been a need for a surgical guideline instead of surgeons' own experience for selecting these geometric parameters. Although implications from peripheral artery studies are useful in the coronary region, substantially different shear patterns are expected in typical physiological coronary flow conditions, and thus different conclusions would be reached. Furthermore, none of the above-mentioned studies has been sufficient to analyze completely the causative hemodynamic parameters. The aim of this study is therefore to systematically investigate the effects of several critical geometric factors on hemodynamics in coronary anastomoses. To achieve this, numerical simulation is still one of the most attractive approaches for parameters can be easily altered and highly-resolved results of the entire flow field for complex geometries can be obtained. The numerical method also makes it possible and easy to calculate complicated wall parameters such as OSI, spatial and temporal shear gradients. As discussed in Chapter 4, low cycle-averaged WSS, high OSI and high SWSSG are dominating factors causing lesion development in the distal coronary anastomoses and therefore will be used as primary indicators of nonuniform hemodynamics for predicting lesion susceptible sites and assessing model performance. Detailed flow characteristics at different phases of a cardiac cycle are also analyzed as they can reflect directly the effects of parametric changes, e.g. model geometry and input flow waveform, on the behavior of blood flow. 


\subsection{FLOW CONDITIONS}

The modeling was conducted under typical physiological coronary flow conditions. A fully-developed pulsatile velocity profile was applied at the graft inlet boundary. The velocity profile in most large arteries is more or less blunt rather than well-developed. There is evidence, however, that using a fully-developed velocity profile at the inlet does not produce significant difference in the arterial velocity and wall shear stress patterns in studying coronary artery blood flow, from using a blunt or a Dean-type profile (Myers et al., 2000). In all models, the graft inlet boundary is located at approximately 12 graft diameters from the distal anastomosis, which was considered to be sufficiently long to obtain a stable flow field and WSS at the anastomotic region. The input waveforms as shown in Fig. 5.1 were obtained from Kajiya et al. (1986) and Matsuo et al. (1988), simulating flows in the LAD and RCA systems, respectively. Based on the host artery diameter $\left(d_{a}\right)$, both waveforms give a mean Reynolds number of 230. The pulse rate was set as 75 beats per minute, simulating a resting condition for human beings (McDonald, 1974). The models contain a proximal and a distal outlet boundary in the host artery and a 20:80 proximal-distal flow division ratio was specified. The proximal and distal outlet segments were extended about 20 artery diameters, from the center of the anastomotic region to ensure a stable and perturbationfree anastomotic flow field. At the two outlet boundaries, the conditions describing zero surface traction were assumed. 

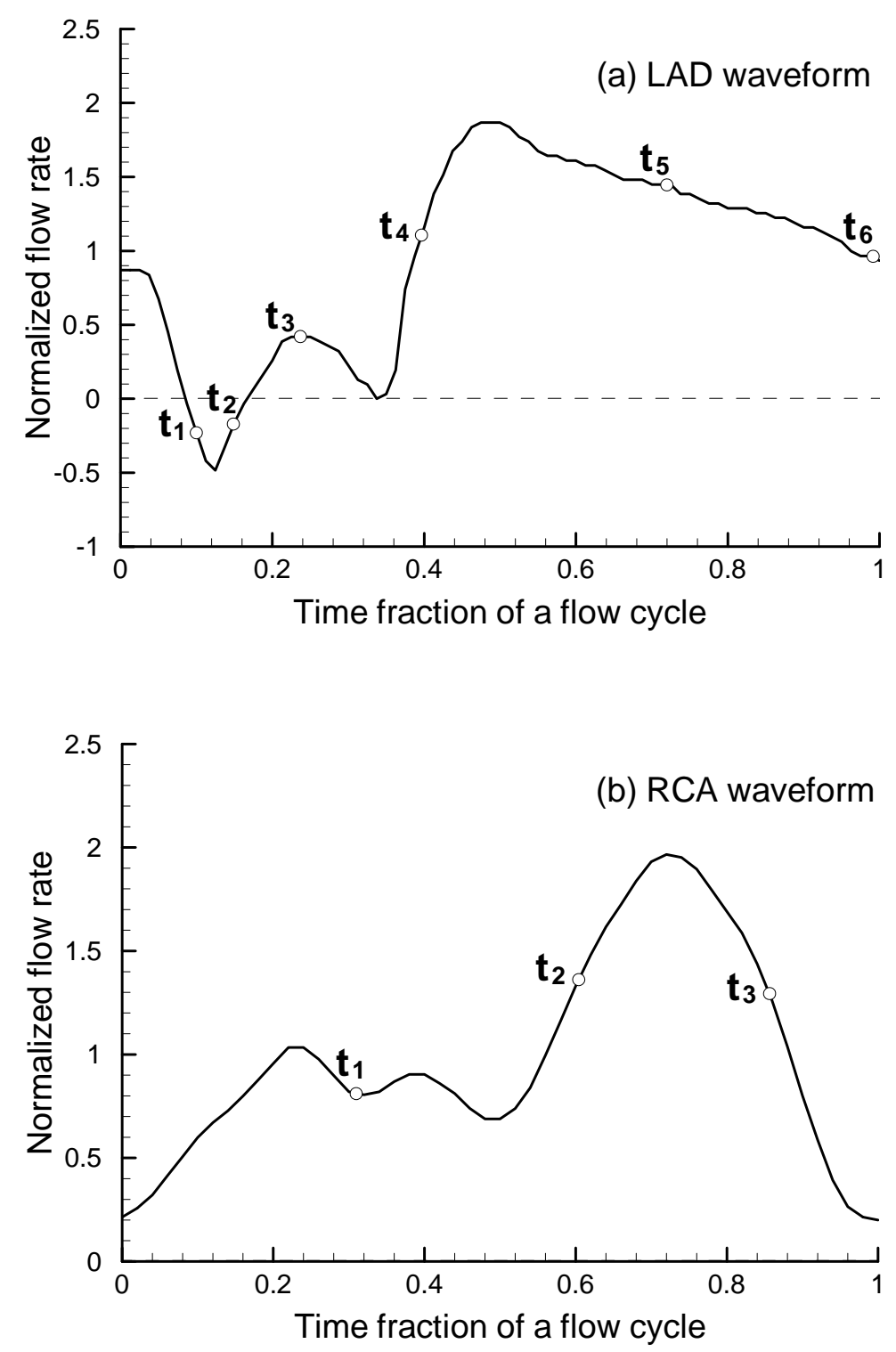

Fig. 5.1 The flow waveforms used in the study based on the work of Kajiya et al. (1986) and Matsuo et al. (1988). Labels indicate time steps at which flow patterns are presented. (a) LAD waveform: early negative flow $\left(t_{1}\right)$, late negative flow $\left(t_{2}\right)$, mid systole $\left(t_{3}\right)$, diastolic acceleration $\left(t_{4}\right)$, mid diastolic deceleration $\left(t_{5}\right)$, and late diastolic deceleration $\left(\mathrm{t}_{6}\right)$; and (b) RCA waveform: mid systole $\left(\mathrm{t}_{1}\right)$, diastolic acceleration $\left(\mathrm{t}_{2}\right)$, and diastolic deceleration $\left(t_{3}\right)$. 


\subsection{EFFECT OF GRAFT-ARTERY DIAMETER RATIO}

\subsubsection{Numerical Model}

The coronary artery in adults varies between 1.5 and $5 \mathrm{~mm}$ depending on gender, age and location (Dodge et al., 1992). The saphenous vein graft is usually larger than the native coronary artery, whereas the arterial grafts (e.g. internal mammary artery and internal thoracic artery) have diameters comparable to the coronary artery (Reardon et al., 1997). In this section, three graft-artery diameter ratios $(\Phi=1: 1,1.5: 1$ and $2: 1)$ were investigated with the graft-artery angle fixed at $30^{\circ}$. When constructing the models with the diameter ratio larger than $1: 1$, the distal end of the graft was flattened with a long and narrow elliptic-shape cross-section in order to meet with the horizontal diametric plane of the smaller host artery. Figure 5.2 shows the typical model geometry with relevant terminology. Based on the density determined by the mesh-independence study in Chapter 3, the models consist of 108,936 to 148,816 hexahedral cells (the mesh size increases with increased complexity of model geometry). Biased accumulation of cells was applied at the anastomotic region where most complex flow is anticipated.

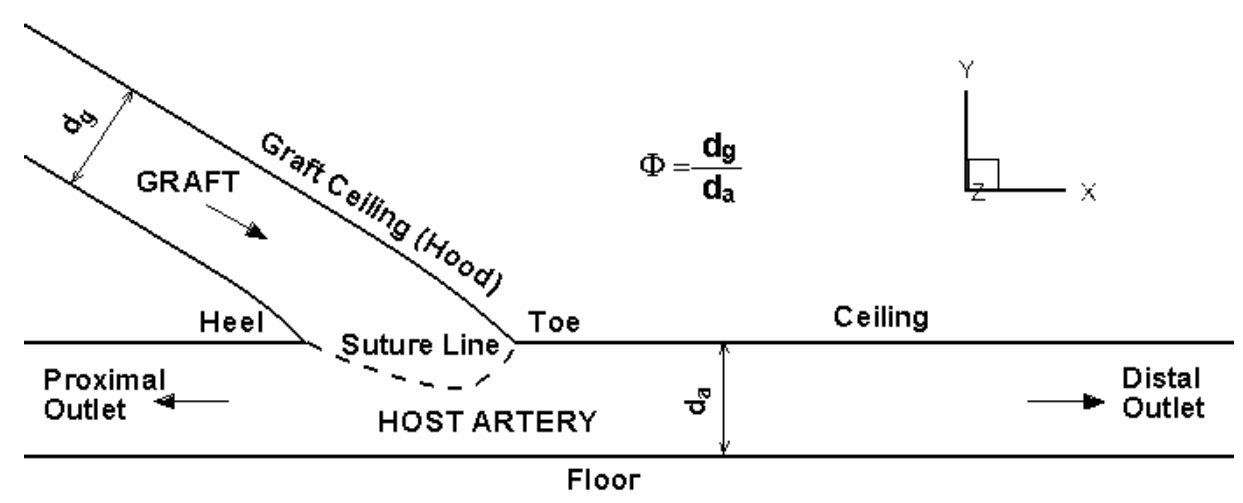

Fig. 5.2 Geometric configuration of the model with relevant terminology. 


\subsubsection{Results: LAD Waveform}

Flow characteristics having critical effects on shear stress patterns are summarized and compared in Fig. 5.3 through 5.9 at six typical time steps of the LAD waveform as labeled in Fig. 5.1a. During the early negative flow phase (Fig. 5.3), the lowmomentum fluid near the graft wall that is more easily driven by the external pressure becomes already retrograde, whereas the higher-inertia fluid in the mainstream have not decayed from the previous part of the cardiac cycle and remains forward. A cohesive vortex, mixing a portion of the forward flow from the graft and the retrograde flow from the proximal artery, is developed in the model with diameter ratio of $1: 1$, with its core located in the middle of the anastomotic region. The formation of the vortex, in turn, produces the most complex flow structures at the entrance of the distal artery in the 1:1 model, showing mostly retrograde flow in the upper part of the vessel and a small portion of forward flow in the lower part. Consequently, three stagnation points (i.e. zero-shear point) are formed on the artery floor, which are located at around $\mathrm{X}=$ $1.6 d_{a}, 2.6 d_{a}$ and $-0.4 d_{a}$, respectively as indicated in Fig. 5.3. Increasing the diameter ratio to $1.5: 1$ and $2: 1$ causes the vortex to be attenuated and the overall flow velocity in the graft and the anastomotic region to be reduced. This is expected since, given a constant volume flow rate, the flow in a larger space should possess a lower mean momentum. During the late negative flow (Fig. 5.4), the flow in the graft of the 1:1 model becomes totally retrograde under the action of sustained adverse pressure gradient, but not yet in the $1.5: 1$ and 2:1 models. The main vortex in the $1: 1$ model is cleared but flow separation occurs along the graft walls just upstream of the toe and the heel. In the 1.5:1 and 2:1 models, no sign of flow separation is shown around the toe, whereas the separation around the heel is appreciable. Flow disturbances are attenuated at the mid systole and during the diastolic acceleration (Fig. 5.5 and 5.6). The diastolic 
deceleration phase promotes flow disturbances again (Fig. 5.7). The 1:1 model exhibits severe flow skewing at the entrance of the distal artery segment, exerting strong impinging forces on the floor and diverting the flow away from the artery ceiling. A small flow recirculation near the heel is also recognizable in this model. All these flow features are sustained till the end of diastole (Fig. 5.8). Figure 5.9 shows secondary flows in different flow phases at the cross-section of the host artery about $1 d_{a}$ downstream of the toe. The secondary flow is promoted when the flow begins to decelerate and is flow rate dependent, e.g. is the highest at the peak flow rate. The flow skewing and secondary flows are substantially suppressed in the 1.5:1 and 2:1 models.

Differences in flow patterns are reflected in WSS distributions (Fig. 5.10). In the 1:1 model, extensively low WSS (with the normalized value below 0.5 ) characterizes the ceiling of the distal artery immediately downstream to the toe. This low shear region is almost eliminated in the 1.5:1 and 2:1 models. Low WSS also appears in small areas on the lateral wall of the graft-artery interface and around the graft ceiling in the 1:1 model. Increasing the diameter ratio to $1.5: 1$ causes the majority of the graft to be exposed to low shear. In the 2:1 model, the low-shear area extends further to cover more than the proximal half of the native artery segment adjoining the graft orifice (from $X=-0.2$ $d_{a}$ to $1.8 d_{a}$, about $2 d_{a}$ in length). In addition, the WSS is low along the entire proximal artery outlet segment for all the models investigated.

Since the OSI is defined based on low and oscillatory shear, the regions with high OSI closely resemble those with low WSS (Fig. 5.11). In the 1:1 model, high OSI characterizes the ceiling of the native artery near the toe and heel, the lateral wall of the graft-artery interface, and the artery floor opposite the graft orifice. Increasing the ratio to 1.5:1 removes high OSI at the toe and reduces the high-OSI area at the heel, but on 
the other hand increases slightly the OSI level on the graft. Further increasing the ratio to $2: 1$, however, substantially increases the OSI levels on the graft and on the native artery segment adjoining the graft orifice.

The variations of SWSSG and TWSSG on the floor over both space and time are illustrated in Fig. 5.12 and 5.13, respectively. Extremely high values of both SWSSG and TWSSG characterize the 1:1 model. The elevated SWSSG covers a long segment of more than $5 d_{a}$ in length along the floor opposite the graft orifice, with the maximum value of 32.77 delivered to $\mathrm{X}=1.27 d_{a}$, slightly proximal to the toe. The magnitude of the spatial gradient gradually diminishes to zero with axial distance away from the anastomotic region. Increasing the diameter ratio from $1: 1$ to $1.5: 1$ dramatically reduces the peak magnitude of spatial gradient from 32.77 to 9.80 , and the area of the high-gradient region is also accordingly reduced. However, the change by further increasing the diameter ratio to $2: 1$ is not as significant (from 9.80 to 7.14 ). The spatial gradient is flow rate dependent, e.g. is higher in the diastolic phase than in the systolic phase and reaches maximum at the peak flow (at about $1 / 2$ of the flow cycle). For the variations of TWSSG in the 1:1 model, the region with elevated values originates from $\mathrm{X}=-1 d_{a}$ and extends distally to cover the whole distal artery floor segment. The temporal gradient is again substantially lower in the 1.5:1 and 2:1 models as compared to the 1:1 case. For all models, the temporal gradient reaches the maximum at about $2 / 5$ of the flow cycle where the curve of the flow waveform has the highest slope. Figure 5.14 directly compares the variations of cycle-averaged SWSSG on the floor between the three models. Note that the peak magnitude in the $1: 1$ model is a factor of $3 \sim 4$ larger than that in the 1.5:1 model. 


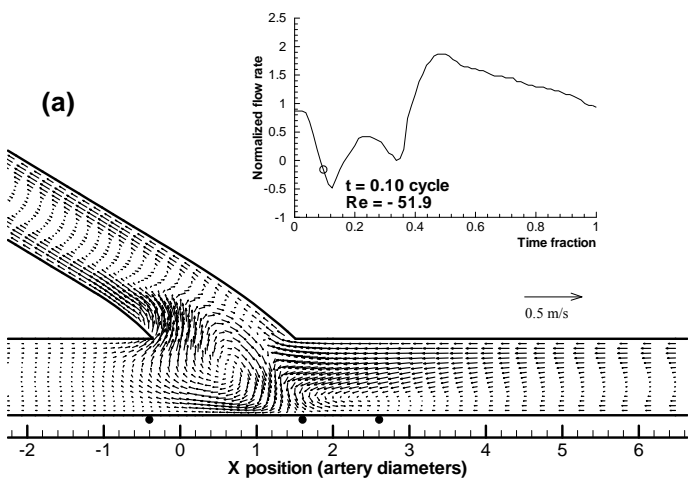

(b)

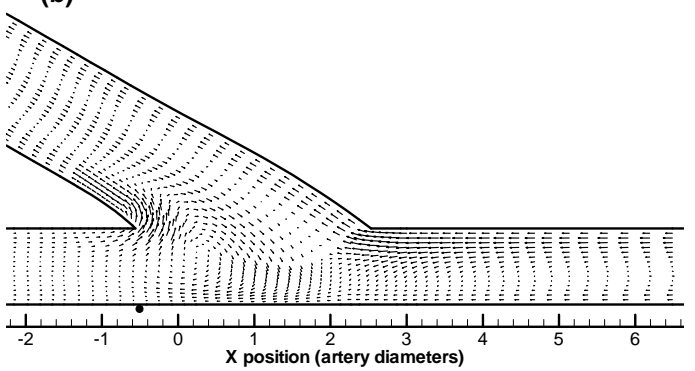

(c)

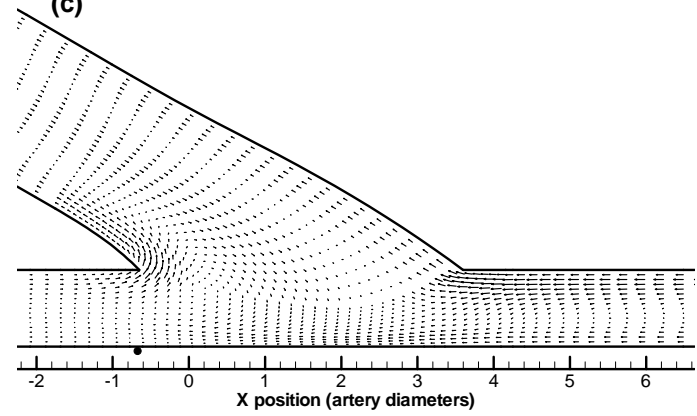

Fig. 5.3 Velocity vector plots in the symmetry plane during early negative flow for the models with (a) $\Phi=1: 1$, (b) $\Phi=1.5: 1$, and (c) $\Phi=2: 1$ in the LAD flow conditions. The black spots indicate positions of flow stagnation points.
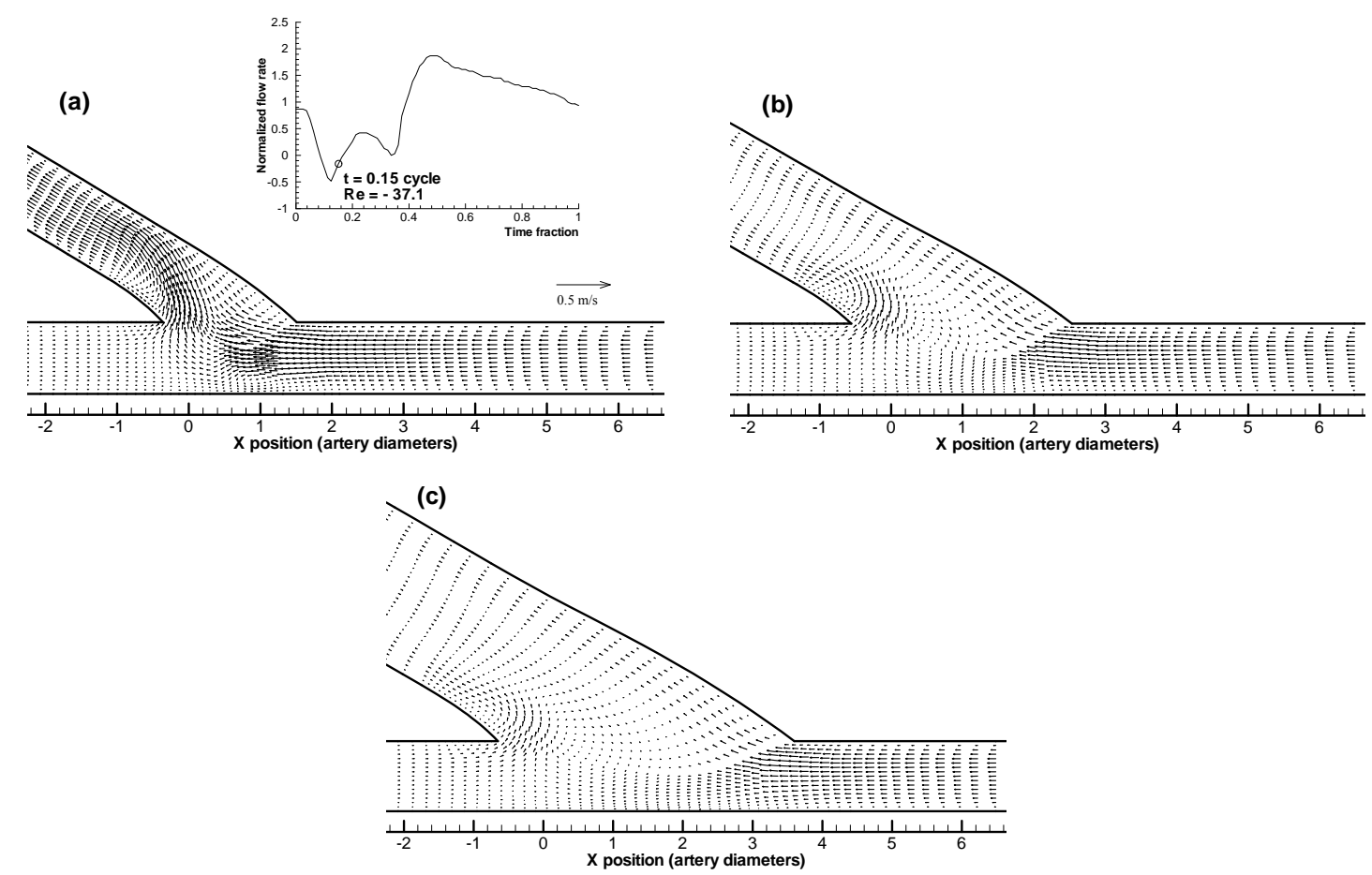

Fig. 5.4 Velocity vector plots in the symmetry plane during late negative flow for the models with (a) $\Phi=1: 1$, (b) $\Phi=1.5: 1$, and (c) $\Phi=2: 1$ in the LAD flow conditions. 

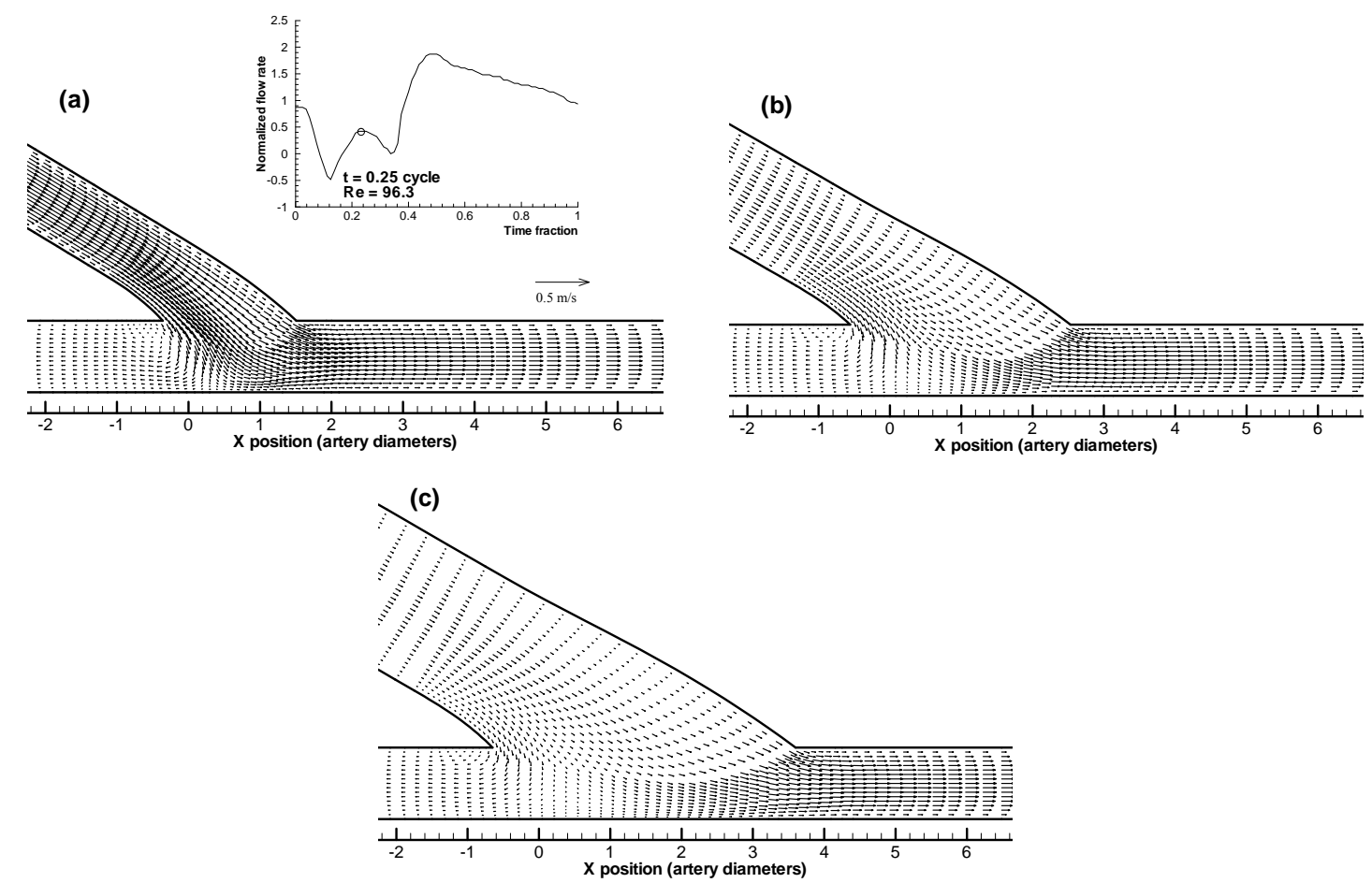

Fig. 5.5 Velocity vector plots in the symmetry plane during mid systole for the models with (a) $\Phi=1: 1$, (b) $\Phi=1.5: 1$, and (c) $\Phi=2: 1$ in the LAD flow conditions.

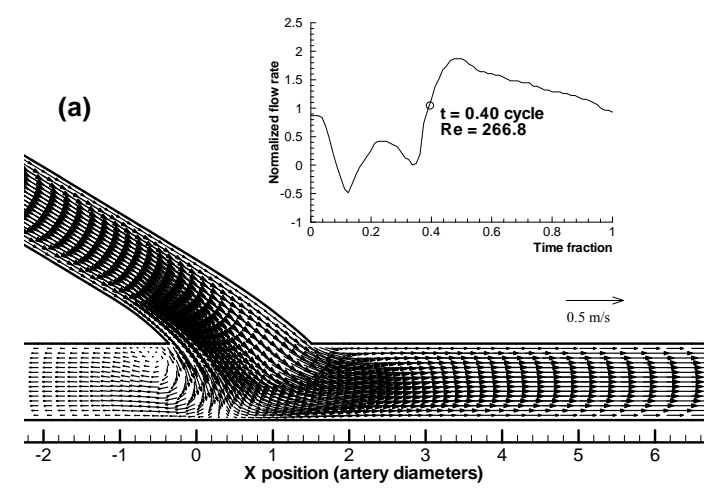

(b)

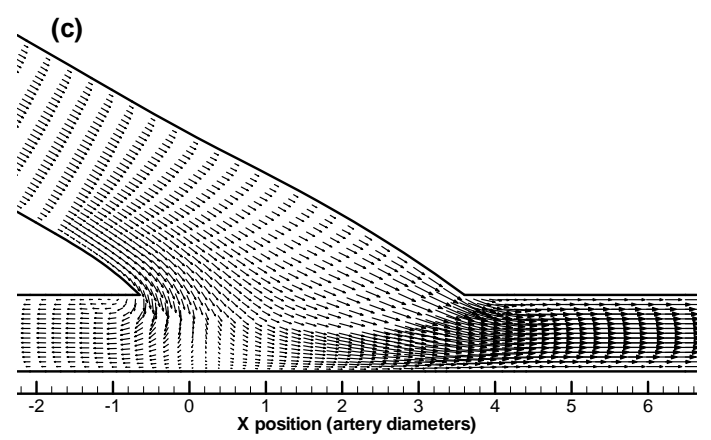

Fig. 5.6 Velocity vector plots in the symmetry plane during diastolic acceleration for the models with (a) $\Phi=1: 1$, (b) $\Phi=1.5: 1$, and (c) $\Phi=2: 1$ in the LAD flow conditions. 


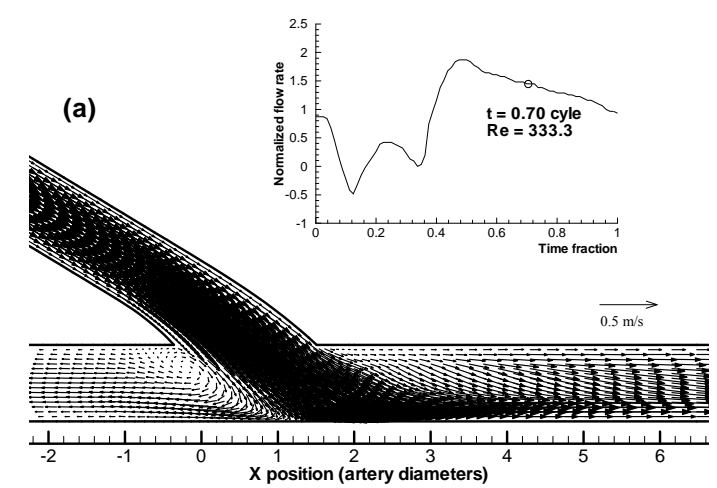

(b)

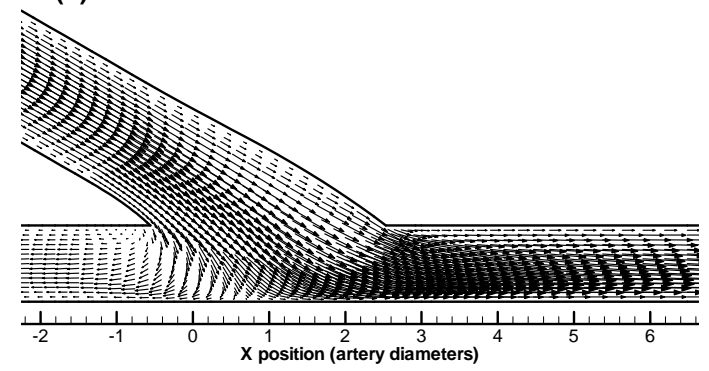

(c)

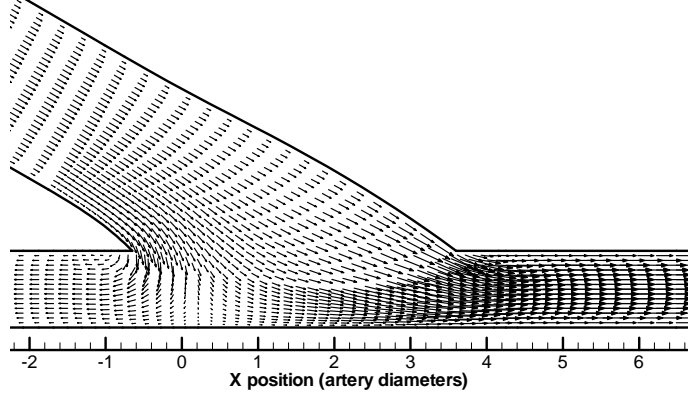

Fig. 5.7 Velocity vector plots in the symmetry plane during mid deceleration for the models with (a) $\Phi=1: 1$, (b) $\Phi=1.5: 1$, and (c) $\Phi=2: 1$ in the LAD flow conditions.
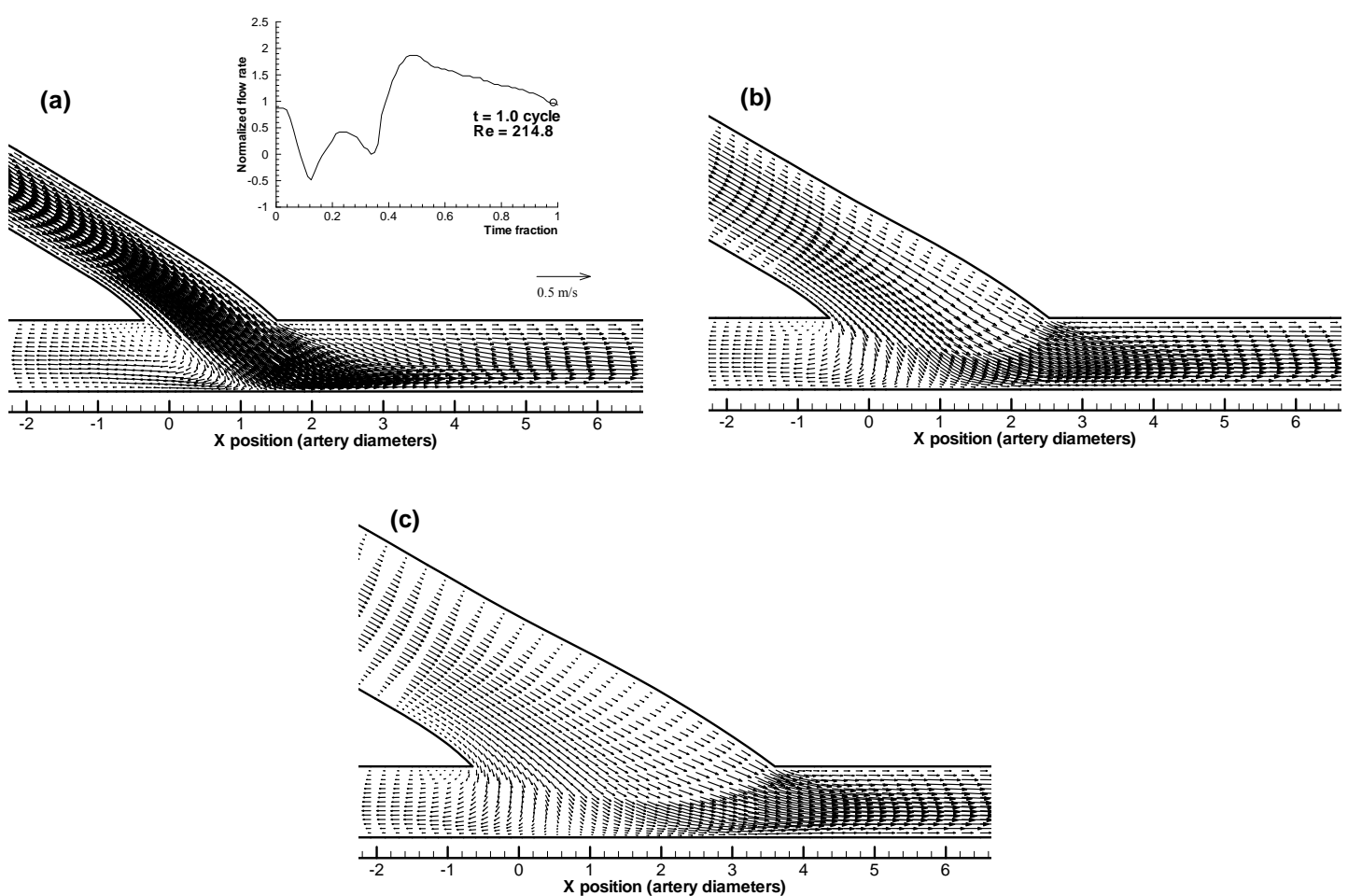

Fig. 5.8 Velocity vector plots in the symmetry plane during late deceleration for the models with (a) $\Phi=1: 1$, (b) $\Phi=1.5: 1$, and (c) $\Phi=2: 1$ in the LAD flow conditions. 
(a)

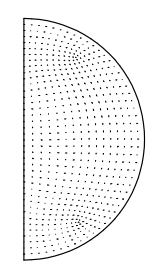

(b)

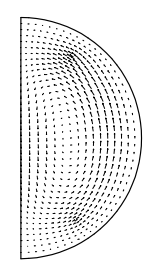

(c)

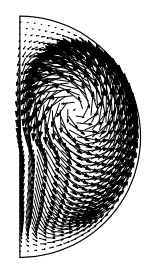

(d)

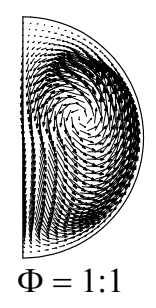

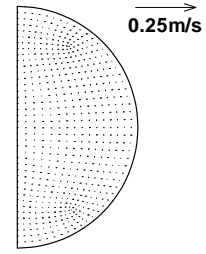
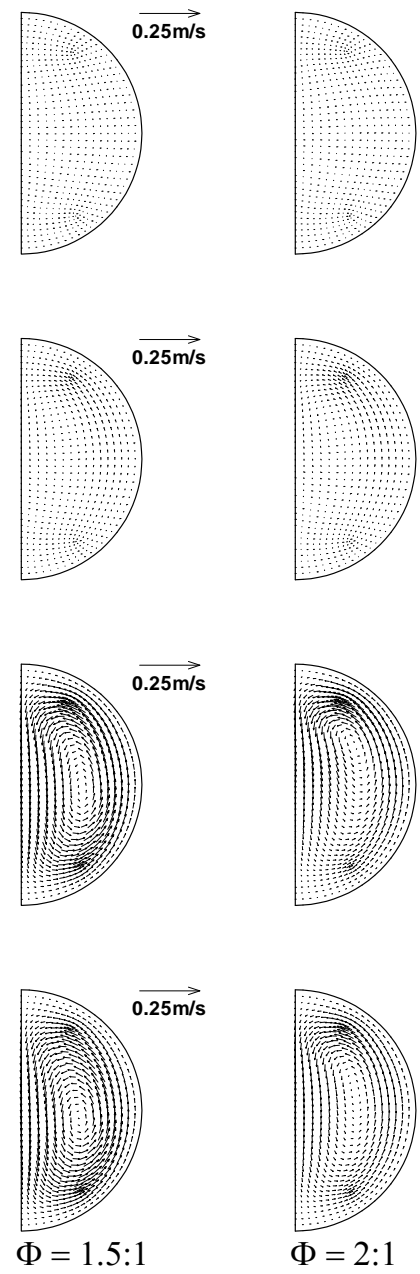

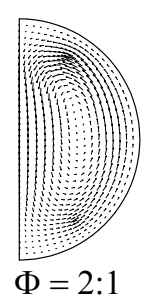

Fig. 5.9 Secondary flows during (a) mid systole, (b) diastolic acceleration, (c) peak diastole, and (d) diastolic deceleration at the cross-section of the host artery about $1 d_{a}$ downstream of the toe for the models with different diameter ratios. 
(a)
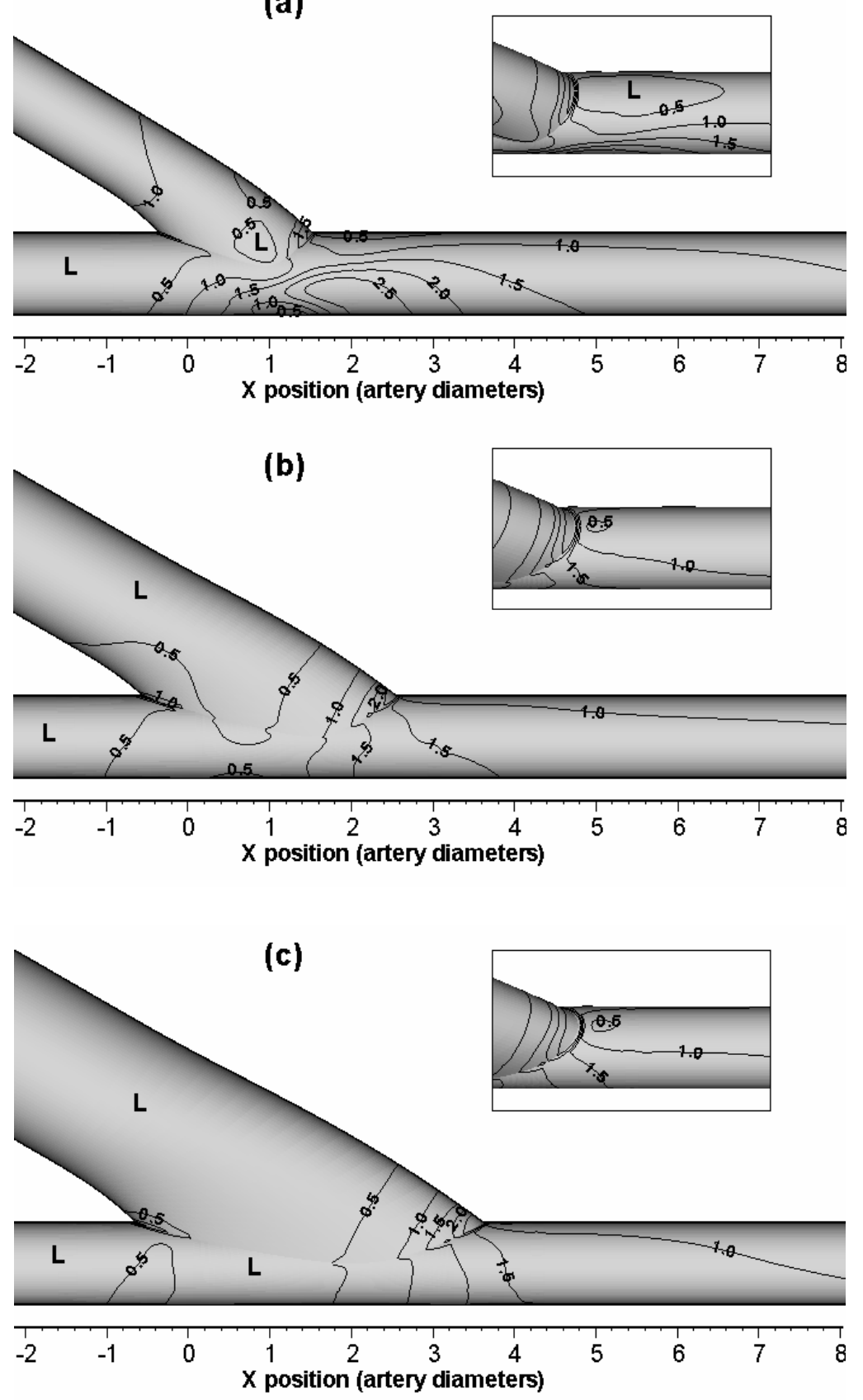

Fig. 5.10 Contours of cycle-averaged normalized WSS for the models with (a) $\Phi=1: 1$, (b) $\Phi=1.5: 1$, and (c) $\Phi=2: 1$ in the LAD flow conditions. The small panel shows the close-up view of the toe. The low shear area with normalized shear magnitude below

0.5 is labeled by "L". 

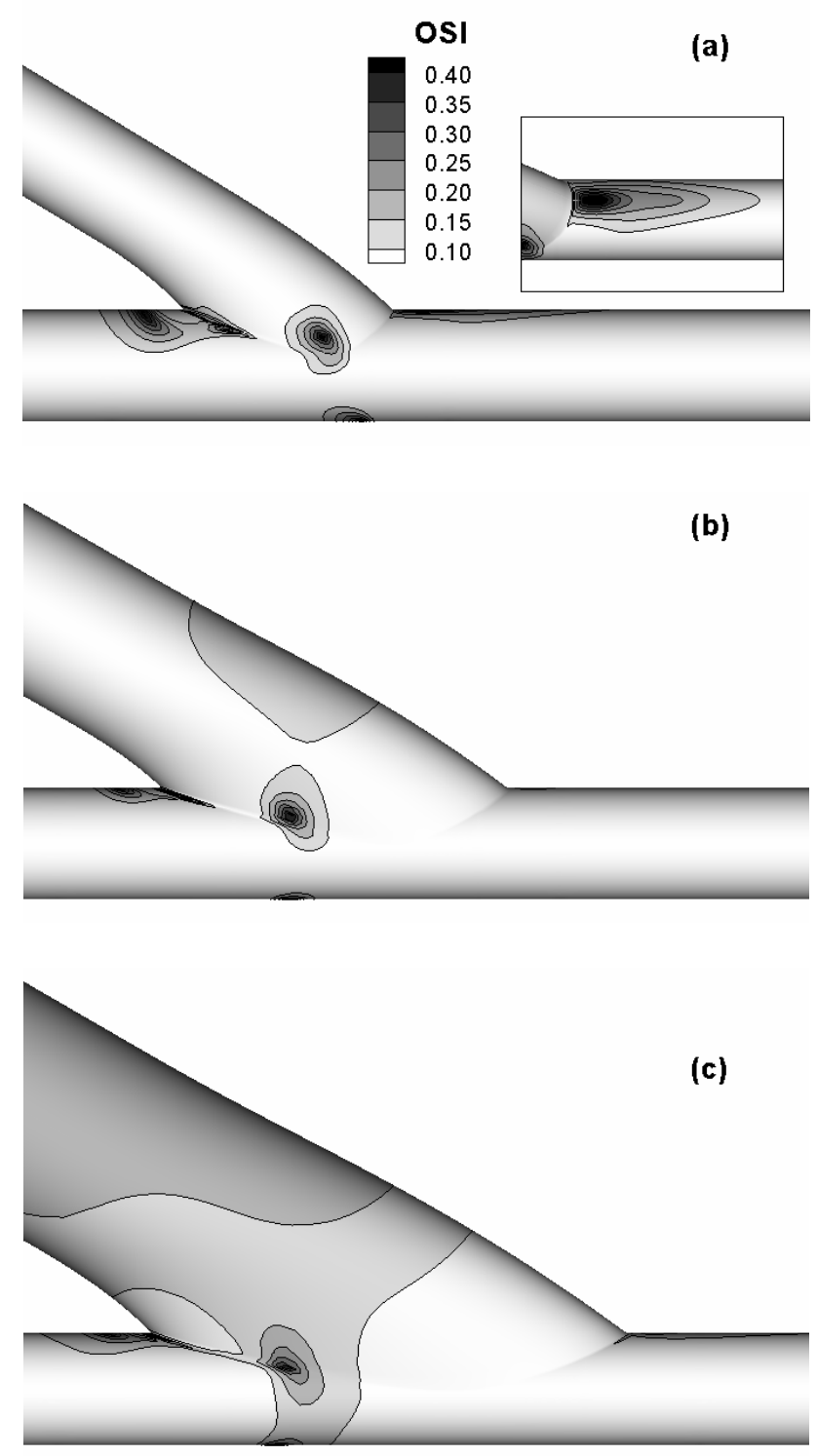

Fig. 5.11 OSI distributions for the models with (a) $\Phi=1: 1$, (b) $\Phi=1.5: 1$, and (c) $\Phi=$ $2: 1$ under the LAD flow conditions. The small panel shows the close-up view of the toe. 


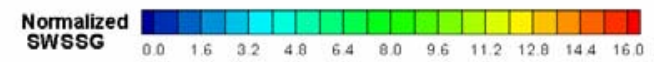

(a)

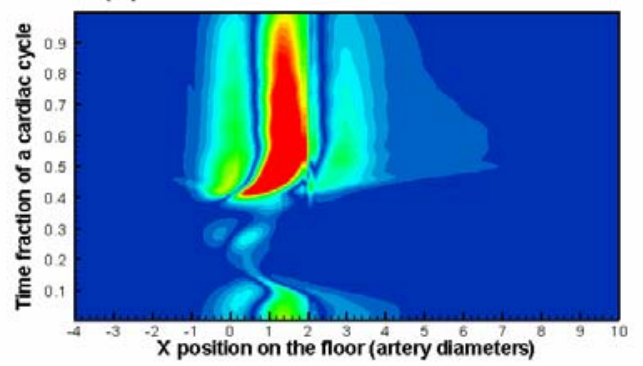

(b)

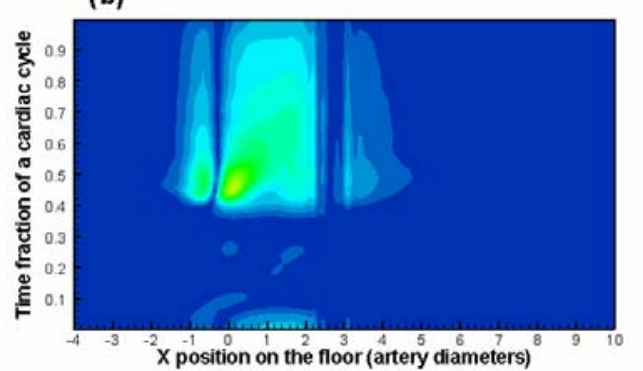

(c)

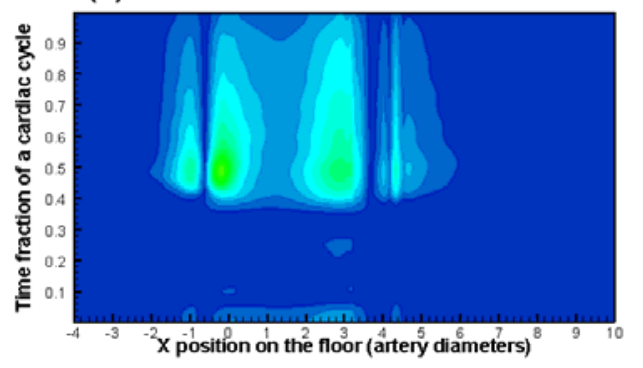

Fig. 5.12 Variations of SWSSG on the artery floor over both space and time in the LAD flow conditions for the models with (a) $\Phi=1: 1$, (b) $\Phi=1.5: 1$, and (c) $\Phi=2: 1$. $\underset{\text { NWSSG }}{\text { Normalized }}$

(a)

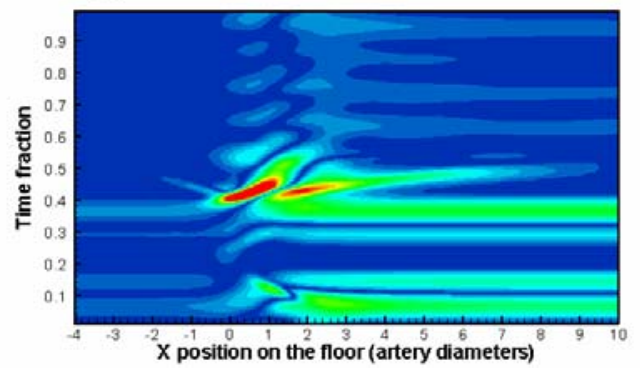

(b)

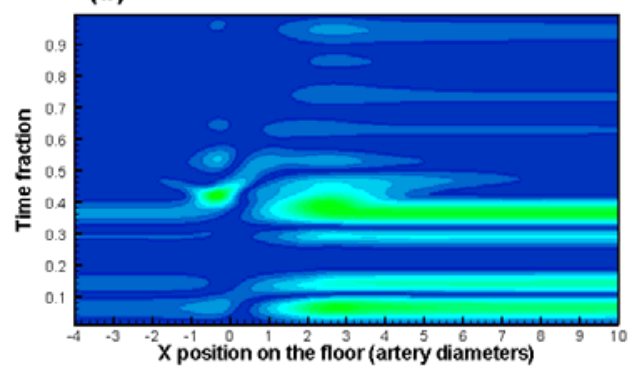

(c)

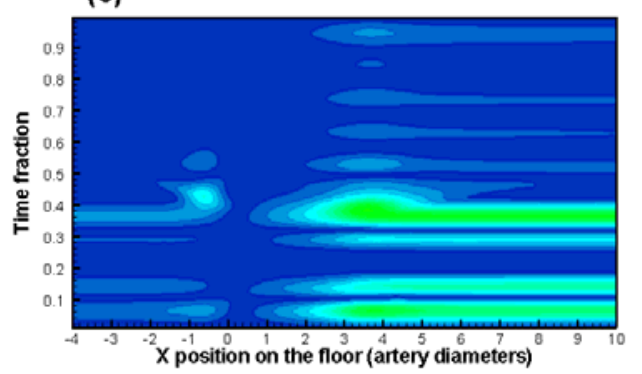

Fig. 5.13 Variations of TWSSG on the artery floor over both space and time in the LAD flow conditions for the models with (a) $\Phi=1: 1$, (b) $\Phi=1.5: 1$, and (c) $\Phi=2: 1$. 


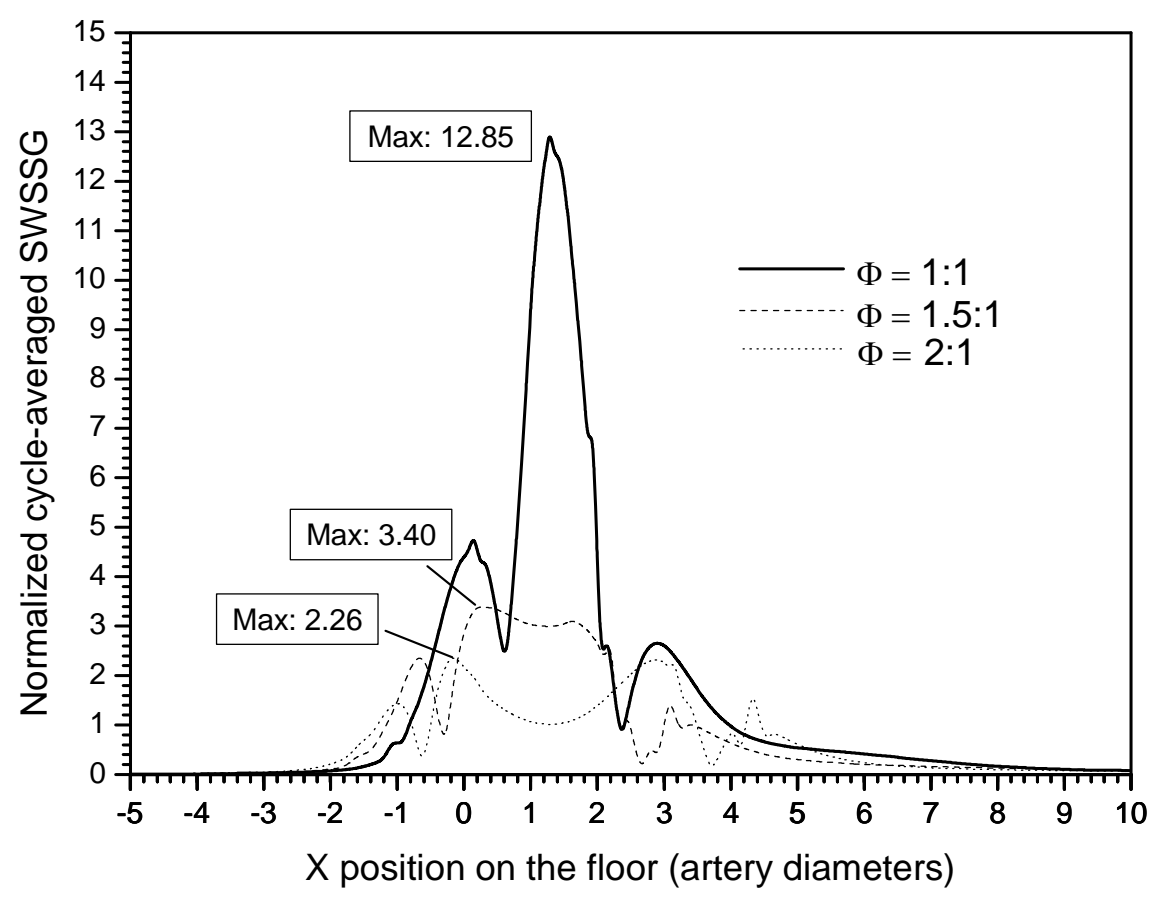

Fig. 5.14 Direct comparison of cycle-averaged SWSSG on the floor between the models with different diameter ratios.

\subsubsection{Results: RCA Waveform}

Figure 5.15 to 5.17 present flow patterns at three typical time steps of the RCA waveform as labeled in Fig. 5.1b. The RCA waveform produces less complex anastomtoic flow fields as compared to the LAD waveform. Local flow disturbances are weak during the systolic phase (Fig. 5.15). However, the characteristic features of separation at the heel and a stagnation point on the artery floor opposite the graft orifice are recognizable in all models. The velocity profile across the vessel at the entrance of the distal artery segment is apparently skewed towards the floor in the model with diameter ratio of 1:1, whereas the profile is nearly symmetric in the models with larger diameter ratios. For the model with diameter ratio of $2: 1$, the entire graft and the proximal part of the anastomotic floor are dominated by low flow. During the diastolic acceleration (Fig. 5.16), the above flow features exhibit no significant change except that the low flow area at the heel of the 1:1 model becomes more prominent. The 
influence of the diameter ratio is pronounced during the diastolic deceleration phase (Fig. 5.17). The high-momentum fluid ejected from the narrow graft of the 1:1 model tends to continue across the vessel, impinging strongly on the artery floor and resulting in an extensive area on the artery ceiling just distal to the toe which is filled with very low flow. This impinging flow affects even the far field of the distal artery segment. Secondary motions in the cross-sections of the distal artery are significant with sufficient magnitude to deliver fluid from the artery lateral wall to the symmetry plane, giving rise to a double helical structure. In contrast, the lower momentum fluid generated by the larger grafts of the 1.5:1 and 2:1 models tends to follow the wall contour without being appreciably diverted when negotiating the toe.

Although the transient flow fields for the RCA waveform are distinctively different from those for the LAD waveform, the cycle-average WSS contours for the two waveforms show identical patterns (Fig. 5.18). For example, the 1:1 model exhibits an extensive low shear region along the artery ceiling immediately distal to the toe, whilst more than the proximal half of the 2:1 model is characterized by extremely low WSS.

The overall OSI levels for the RCA waveform are substantially lower than for the LAD waveform, and the localization of high OSI remains unchanged (Fig. 5.19). In the 1:1 model, high OSI covers very small areas at the heel, toe, lateral wall and floor of the anastomotic region, in contrast with the extensive high OSI areas (particularly the area distal to the toe) in the LAD case (Fig. 5.11a). The OSI levels are even lower and almost negligible in the $1.5: 1$ and 2:1 models.

For the RCA waveform, both the localization and the magnitude of high SWSSG on the artery floor for each model are similar to the LAD case (Fig. 5.20). In the 1:1 model, for 
example, the peak magnitude of 33.01 is delivered to $\mathrm{X}=1.32 d_{a}$, very close to the position in the LAD case $\left(\mathrm{X}=1.27 d_{a}\right)$. Note that the elevated TWSSG for the RCA waveform has longer duration than that for the LAD waveform, but the maximum value is lower, e.g. 3.34 for RCA vs. 5.36 for LAD in the 1:1 model (Fig. 5.21). Again, the 1:1 model is distinguished for its substantially higher magnitudes of both SWSSG and TWSSG.
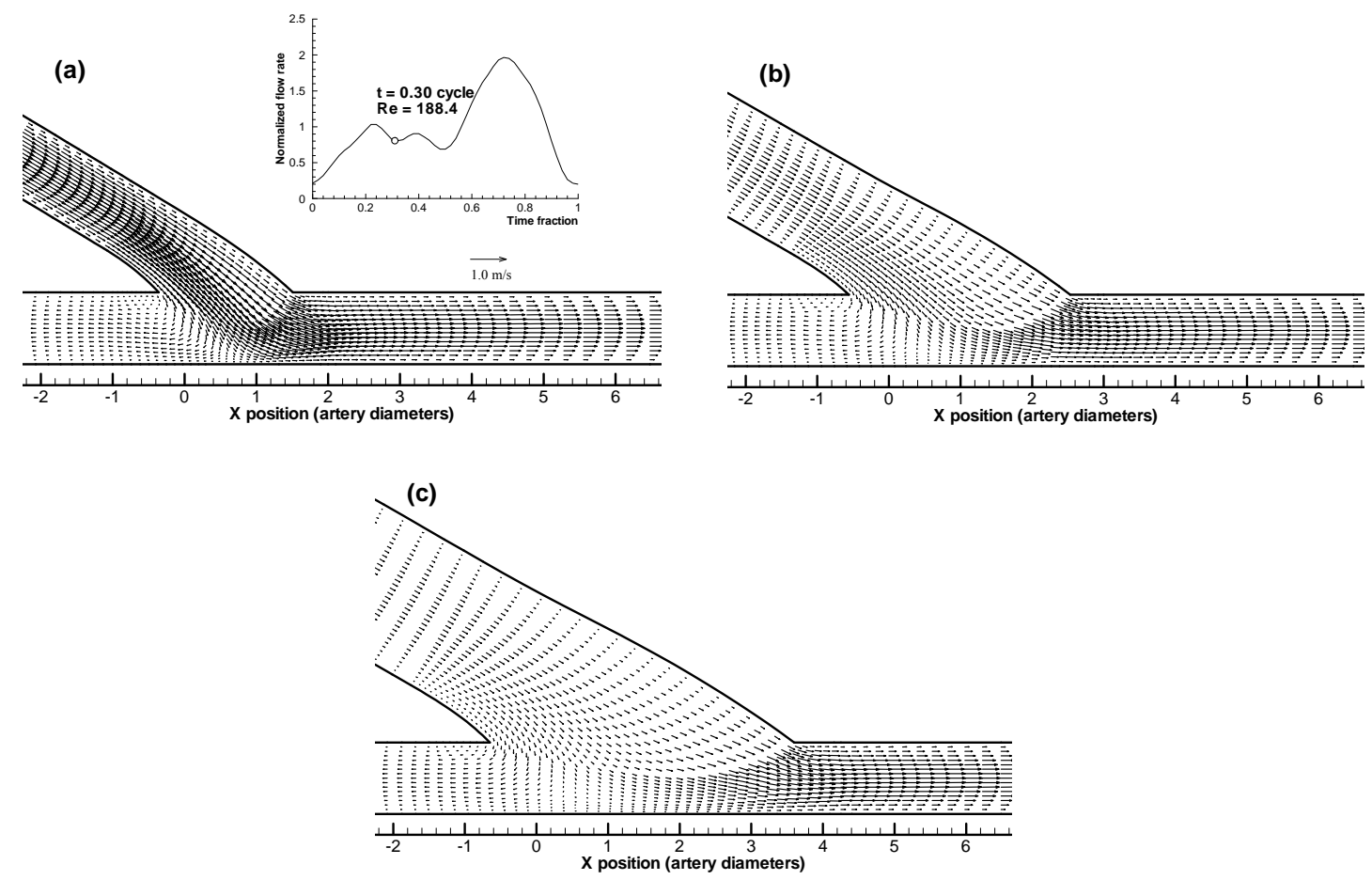

Fig. 5.15 Velocity vector plots in the symmetry plane during mid systole for the models with (a) $\Phi=1: 1$, (b) $\Phi=1.5: 1$, and (c) $\Phi=2: 1$ in the RCA flow conditions. 

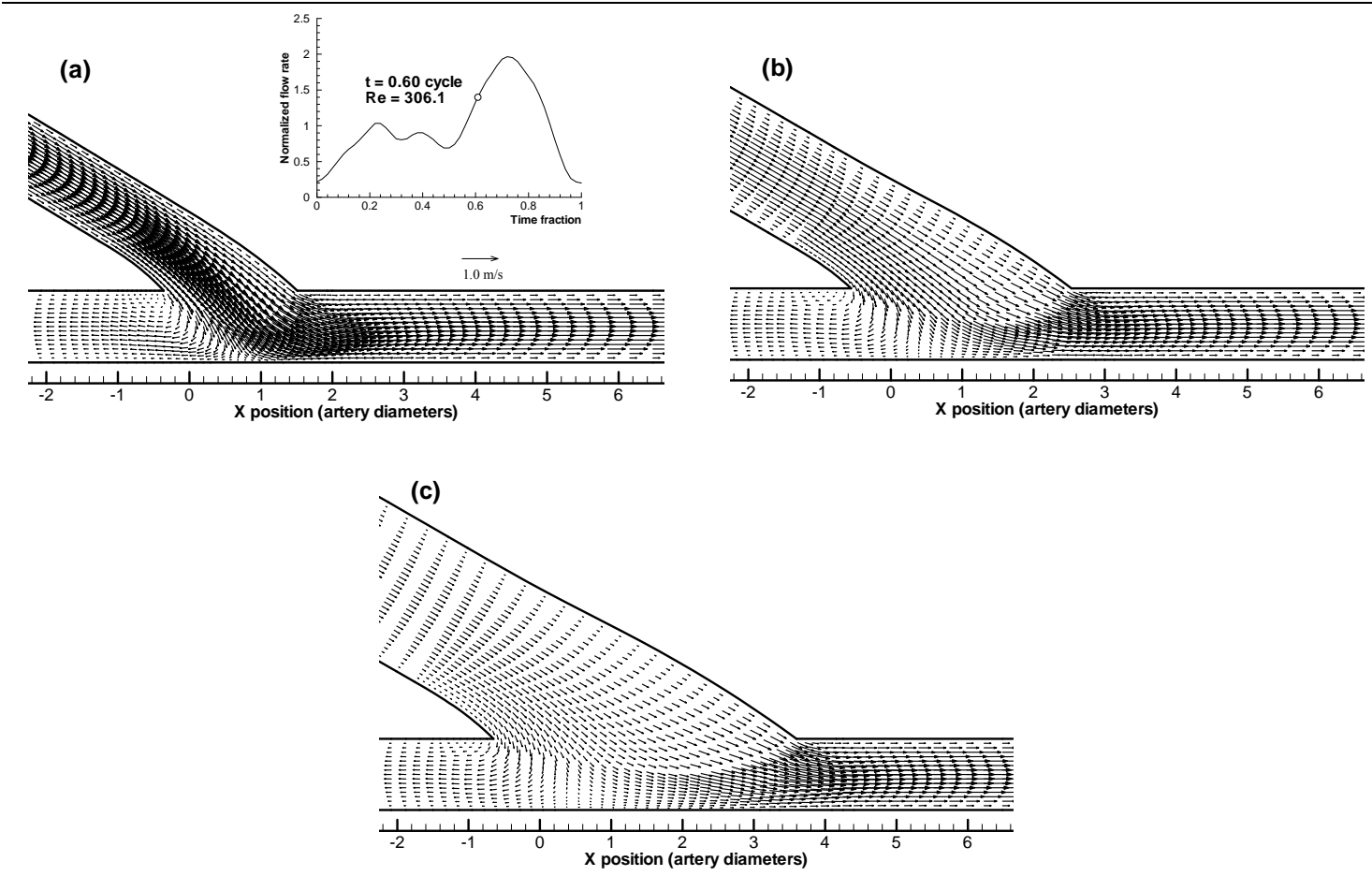

Fig. 5.16 Velocity vector plots in the symmetry plane during diastolic acceleration for the models with (a) $\Phi=1: 1$, (b) $\Phi=1.5: 1$, and (c) $\Phi=2: 1$ in the RCA flow conditions.
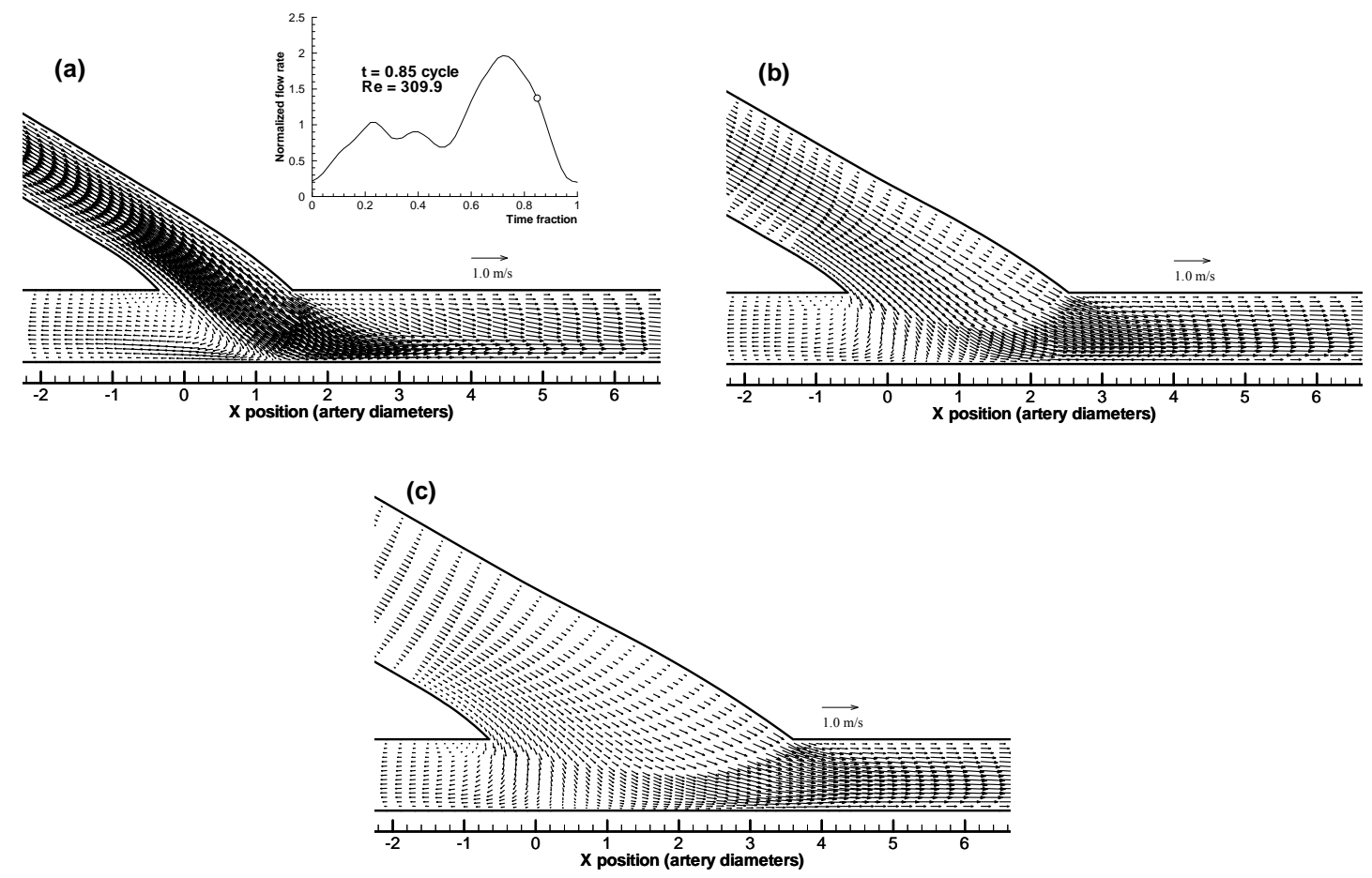

Fig. 5.17 Velocity vector plots in the symmetry plane during diastolic deceleration for the models with (a) $\Phi=1: 1$, (b) $\Phi=1.5: 1$, and (c) $\Phi=2: 1$ in the RCA flow conditions. 
(a)

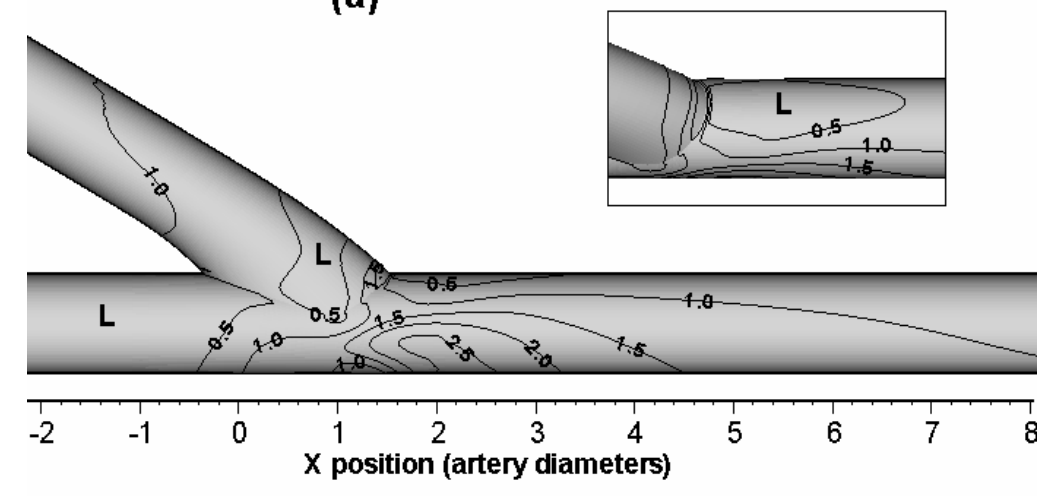

(b)
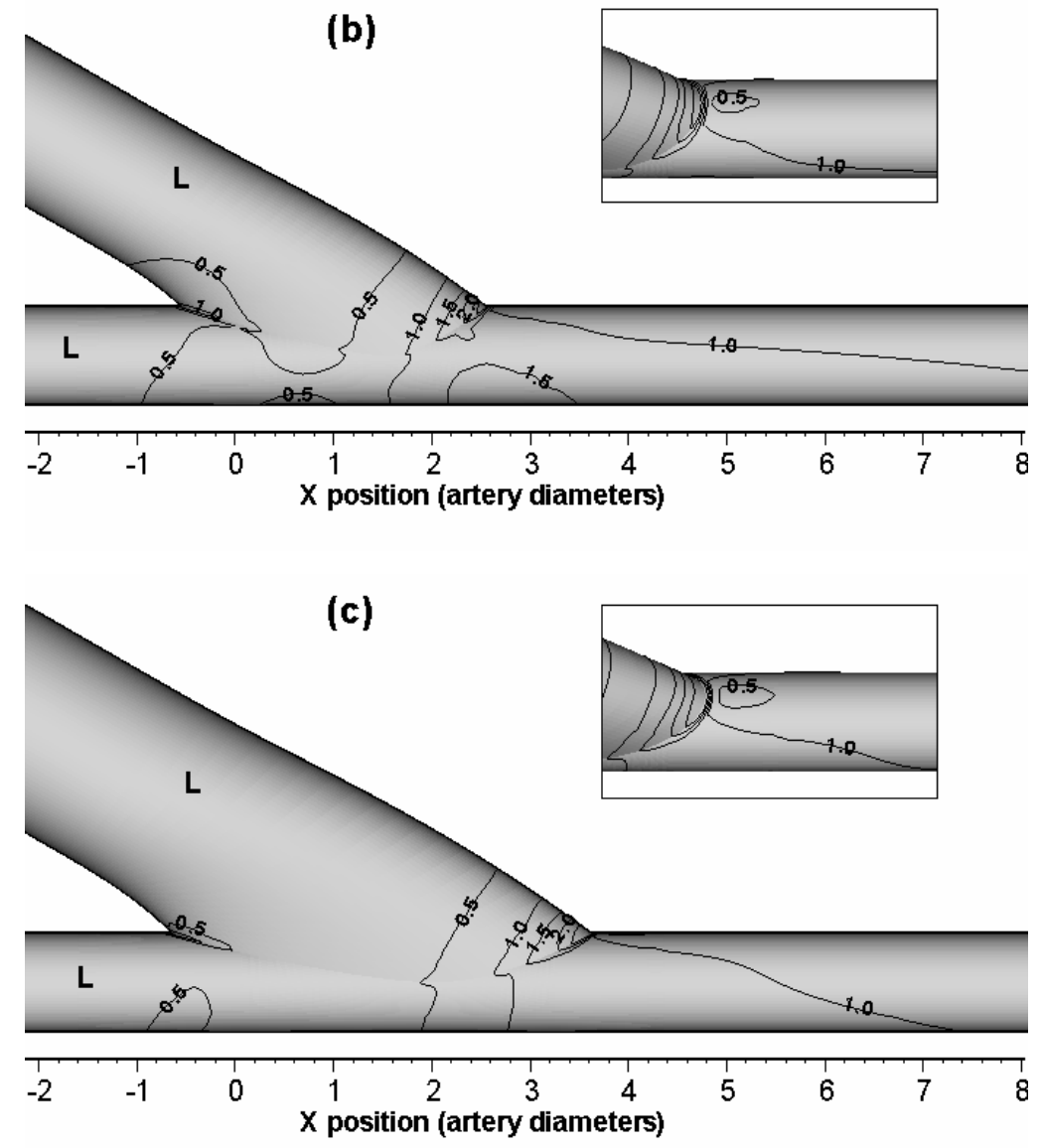

Fig. 5.18 Contours of cycle-averaged normalized WSS for the models with (a) $\Phi=1: 1$, (b) $\Phi=1.5: 1$, and (c) $\Phi=2: 1$ in the RCA flow conditions. The small panel shows the close-up view of the toe. The low shear area with normalized shear magnitude below 0.5 is labeled by " $\mathrm{L}$ ". 

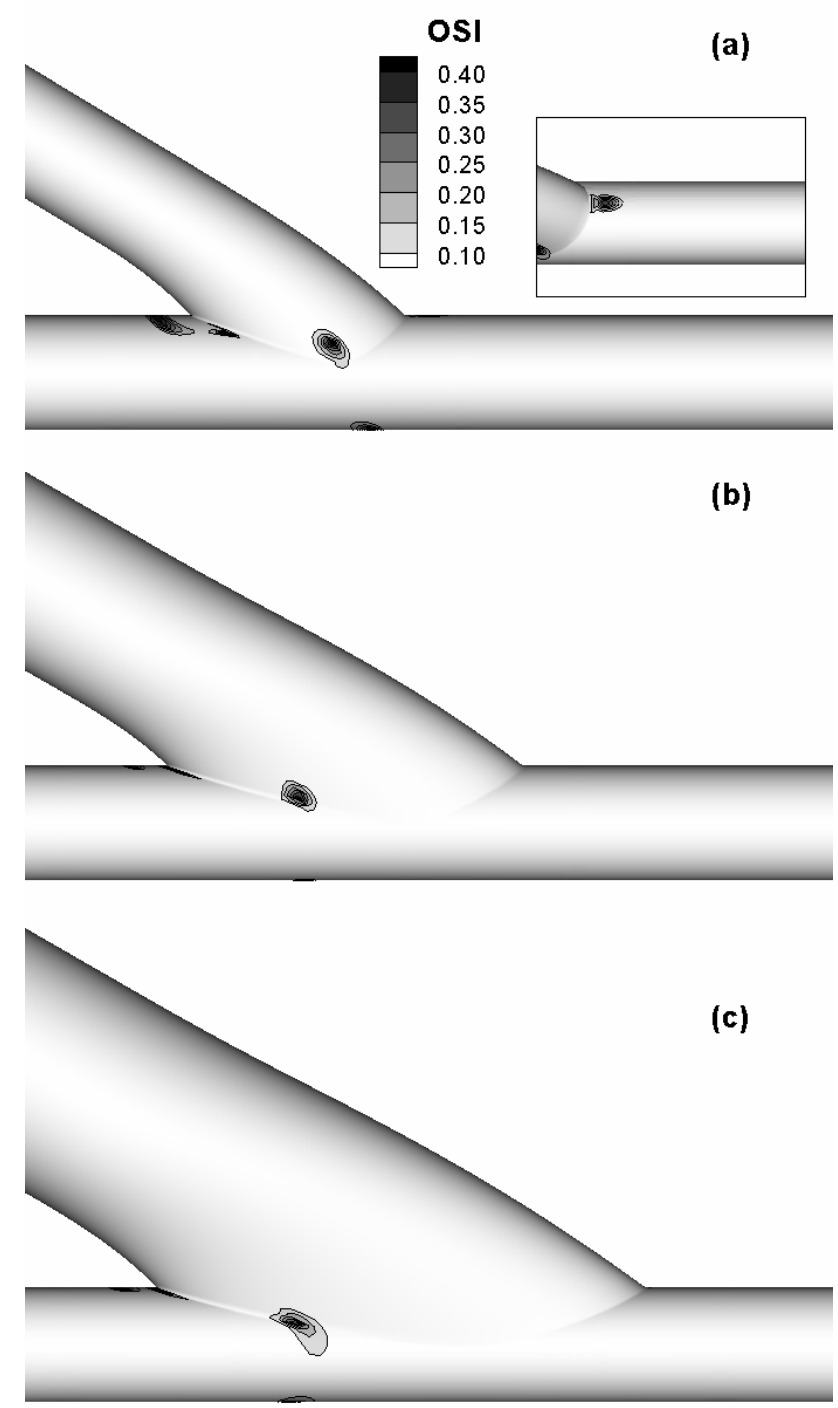

Fig. 5.19 OSI distributions for the models with (a) $\Phi=1: 1$, (b) $\Phi=1.5: 1$, and (c) $\Phi=2: 1$ under the RCA flow conditions. The small panel shows the close-up view of the toe. 


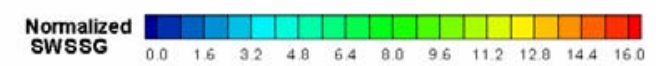

(a)

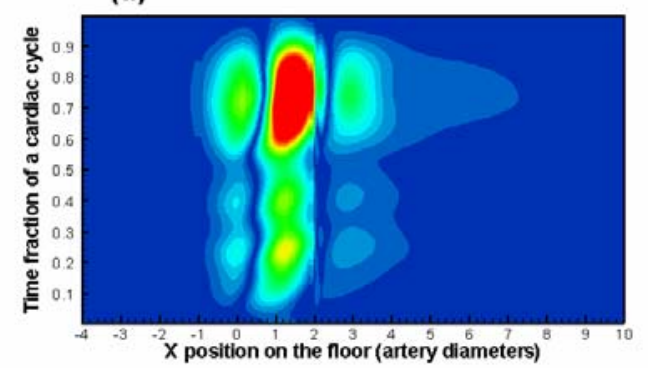

(b)

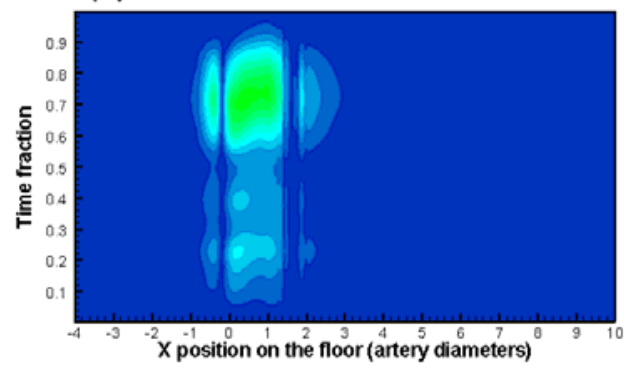

(c)

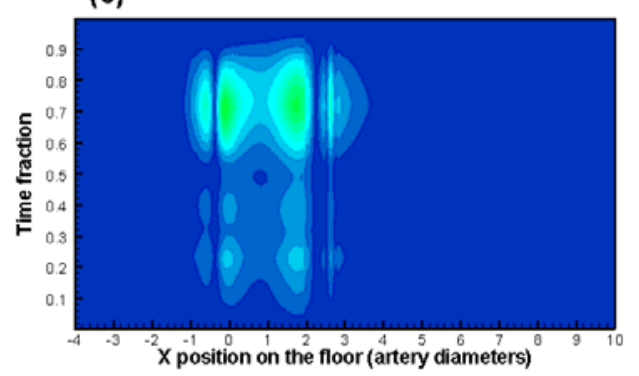

Fig. 5.20 Variations of SWSSG on the artery floor over both space and time in the RCA flow conditions for the models with (a) $\Phi=1: 1$, (b) $\Phi=1.5: 1$, and (c) $\Phi=2: 1$.

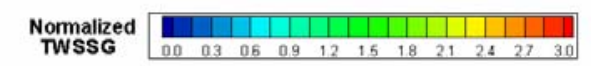

(a)

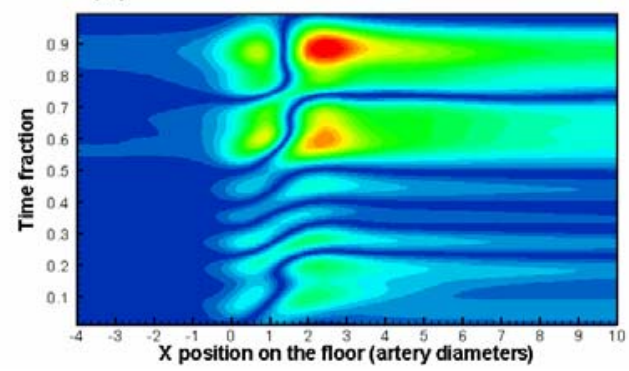

(b)

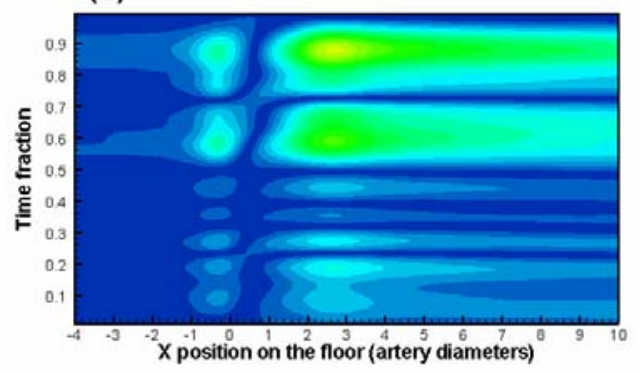

(c)

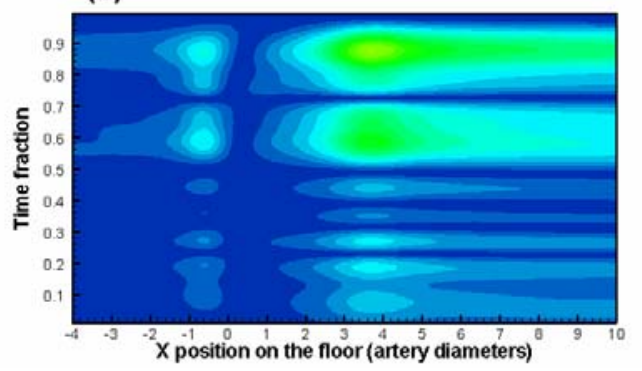

Fig. 5.21 Variations of TWSSG on the artery floor over both space and time in the RCA flow conditions for the models with (a) $\Phi=1: 1$, (b) $\Phi=1.5: 1$, and (c) $\Phi=2: 1$. 


\subsubsection{Discussion}

Despite their clinical relevance and needs, there have been limited studies investigating parametrically the effects of geometric factors upon haemodynamics in distal coronary anastomoses. The information from this study is expected to aid surgeons in selecting appropriate geometric parameters for coronary anastomoses so as to improve hemodynamics and probably enhance long-term performance of anastomosing procedures. This study used as criteria or indicators haemodynamic parameters that have been most frequently implicated in the pathogenesis of the disease, i.e. WSS, OSI, SWSSG and TWSSG.

Correspondence between intimal thickening and sites of low WSS (and particularly, oscillatory shear) has been well documented (Ku et al., 1985; Keynton et al., 2001). Biological studies suggested that low and oscillatory shear stress stimulates intimal and smooth muscle cell proliferative responses by inducing the expression of the plateletderived growth factor (PDGF-A) and messenger RNA (Kraiss et al. 1996), which are critical events involved in the thickening process of intimal hyperplasia (Kraiss and Clowes, 1997). Low flow and low shear magnitude may also increase surface concentration of low-density lipoprotein (LDL), thus promoting the rate of lipid infiltration into the blood vessel (Deng et al., 1995). Although the value of the lower threshold for mean WSS is still not definitive, a number of studies suggested that most intimal thickening occurs where the mean WSS is less than about 10 dynes $/ \mathrm{cm}^{2}$ (Kraiss et al., 1991; Ku et al., 1985) or below one-half that in the native artery (Keynton et al., 2001). In the present study, one-half the normal shear stress in the native artery, i.e. about 12 dynes $/ \mathrm{cm}^{2}$, has been used as the lower shear threshold. The numerical results show that in the model with the graft-artery diameter ratio of 1:1, low and oscillatory 
shear stress (i.e. high OSI) characterize the ceiling of the native artery near the toe and heel, the lateral wall of the graft-artery interface, and the artery floor opposite the graft orifice, where the greatest intimal thickening is anticipated to occur. Increasing the diameter ratio to 1.5:1 almost eliminates both low WSS and high OSI at the toe, and the OSI level at the heel is also accordingly reduced. The toe region is of great importance as the occurrence of thickening there would diminish blood supply to the distal artery and the main vasculatures downstream, which suggests a superiority of the $1.5: 1$ diameter ratio over the $1: 1$ one. Further increasing the ratio to $2: 1$, however, causes most of the native artery segment in the anastomotic region and almost the entire graft to be exposed to substantially higher OSI. This implies that high OSI resulting from extreme diameter mismatch of the graft and host artery, as often seen in coronary anastomoses with saphenous vein grafts, may be part of the reason why atherosclerotic thickening is greater in venous grafts than in arterial grafts (e.g. internal thoracic artery and internal mammary artery) (Ojha et al., 2000; Shelton et al., 1988). More important, there is evidence that endothelial cells even in healed venous grafts fail to serve as a barrier of the blood vessel wall in arterial flow conditions, resulting in a higher tendency of vascular smooth muscle cells and endothelial cells towards proliferation and migration (Berger et al., 1972; Golledge, 1997), and these events can essentially be triggered and favored by low and oscillatory shear (Kraiss et al. 1996). It is therefore implicated that extreme diameter mismatch between the graft and host artery should be avoided specifically for the venous grafts in order to reduce the likelihood of rapid narrowing of the graft lumen. Besides, a large-diameter graft would entail a long incision in the native artery resulting in more suture area and injury to the healthy tissue.

In addition, significant differences in the gradients of WSS induced by altering the graft-artery diameter ratio are noted. The 1:1 model is characterized by remarkably high 
SWSSG and the highest TWSSG on the artery floor. High shear gradients may cause severe deformation and trauma to the local endothelium, thereby breaking down consistent communications between cells and resulting in elevated endothelial permeability to macromolecules (e.g. LDL) which enter the vessel wall by transmural filtration (DePaola et al., 1992; Lei et al., 1995). It is shown that increasing the diameter ratio from $1: 1$ to $1.5: 1$ greatly reduces the peaks of both SWSSG and TWSSG, but the reductions in the same quantities by increasing the ratio from $1.5: 1$ to $2: 1$ are minor. Therefore, the graft should have a diameter at least 1.5 times larger than that of the host artery to avoid extremes in shear gradients. This is in line with the finding of Moore et al. (1999) in their study on femoral artery anastomoses that the floor region in the model with the diameter ratio of $1: 1$ is exposed to much higher spatial shear gradient, with direction of shear fluctuating rapidly during the flow cycle, than is observed in the model with the ratio of 1.7:1. Also, a 1.6:1 diameter ratio was recommended by Lei et al. (1997) to minimize mean spatial shear gradient in femoral anastomoses.

It is also shown that the distribution patterns of the cycle-averaged WSS remain almost unchanged when altering the imposed flow waveform, whereas the OSI for the LAD waveform has a substantially higher level than that for the RCA waveform. The higher OSI level in the LAD case can be attributed to the presence of a negative portion in the LAD waveform, during which the WSS is directed opposite to the mean shear direction. Therefore, it is suggested that the region where the flow waveform has a higher-degree of instability is more prone to atherosclerotic lesions.

This study has demonstrated that the diameter ratio between the graft and host artery is a crucial geometric factor that should be carefully constructed in coronary 
revascularization surgeries. Similar conclusions have been reached by Moore et al. (1999) in their numerical study on femoral-popliteal anastomoses. Based on the specific coronary flow conditions, this study suggests that the graft of a diameter about 1.5 times larger than the host artery would improve hemodynamics. It is recognized that this study used only one flow division ratio in the proximal and distal outlet segments (i.e. 20:80), and changing the division ratio may change the above optimal values of the geometric parameters. For example, an increased portion of distal outflow would impinge more strongly on the artery floor and thus increase shear gradients. In this case, a larger graft-artery diameter ratio may be appropriate to reduce extremes of shear gradients. Whether the flow division ratio, which depends on the severity of the disease and the location of the anastomosis, is significant in affecting the choice of geometric parameters needs further investigation. Although the results in the present study cannot be completely generalized, they at least can serve as a reference for surgeons to select a proper diameter ratio with respect to different flow conditions, during anastomosis construction. 


\subsection{EFFECT OF GRAFT-ARTERY ANGLE}

\subsubsection{Numerical Model}

The anastomotic angle between the graft and host artery $(\alpha)$ is usually small, not more than $70^{\circ}$ in most coronary reconstruction surgeries (Anayiotos et al., 2002). In this study, four angles varying over $15^{\circ}, 30^{\circ}, 45^{\circ}$ and $60^{\circ}$ were investigated with a fixed graft-artery diameter ratio of $\Phi=1.5: 1$. Figure 5.22 shows the typical model geometry with relevant terminology.

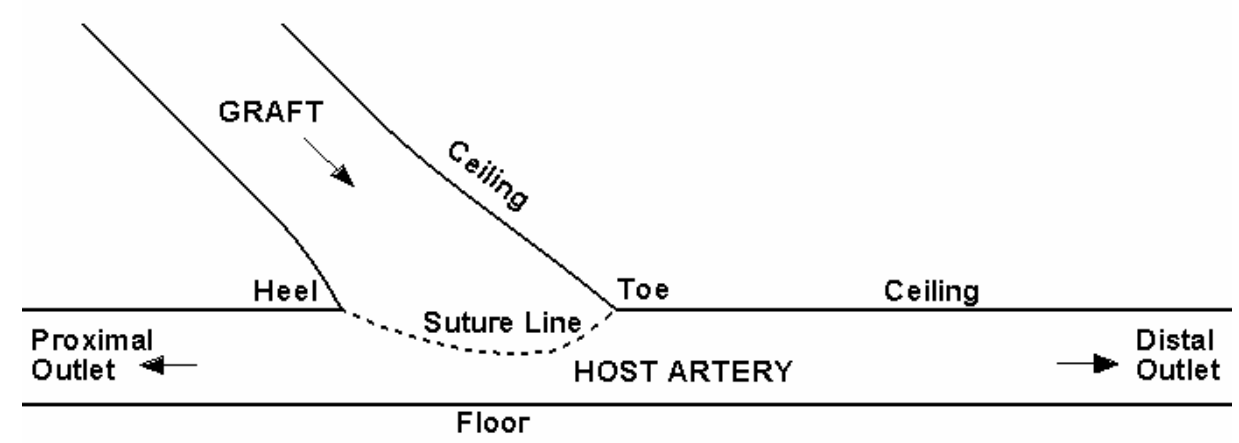

Fig. 5.22 Geometric configuration of the model with relevant terminology.

\subsubsection{Results: LAD Waveform}

The characteristic flow behaviors occurring in the four models with variable angles show no significant qualitative difference. During the early negative flow, all models demonstrate a flow recirculation in the vicinity of the heel. In the distal artery segment, the flow is completely retrograde with skewed M-shape profiles across the vessel. During the late negative flow, the recirculating flow is attenuated as indicated by the reduced length of the velocity vectors, and its core drifts slightly away from the heel. For all the models investigated, flow separation is recognizable along the graft wall just upstream of the heel. At mid systole, the vortex in each model is completely cleared and the flow returns to its primary direction. A region of low-momentum flow appears along the ceiling of the proximal artery next to the heel, and this region slightly 
decreases in size with increasing the angle. This feature persists throughout the remainder of the cycle. In addition, the adverse pressure gradient during diastolic deceleration causes the effect of the impinging flow on the artery floor to be accentuated, particularly in the model with $\alpha=60^{\circ}$.

The overall WSS distribution patterns are similar except that the $15^{\circ}$ model has an apparently lower shear level on the native artery segment adjoining the graft orifice (about $1.0 d_{a}$ in length) (Fig. 5.23). The localization of low shear slightly differs between the four models. In the $15^{\circ}$ model, the lowest WSS covers a large area on the hood and a small area on the lateral wall of the anastomotic region. As the angle increases, the low shear area on the hood gradually shifts towards the region immediately upstream of the heel.

In all models, elevated OSI characterizes the graft body, the heel, the toe as well as the lateral wall and floor of the anastomotic region (Fig. 5.24). The $15^{\circ}$ model has distinctively higher levels of OSI on the graft and the lateral wall of the anastomotic region. Increasing the angle tends to shift high OSI from the graft ceiling to the region just upstream of the heel, but the overall OSI levels for the $30^{\circ}, 45^{\circ}$ and $60^{\circ}$ models are comparable.

The level of SWSSG is comparable for the models of $\alpha=15^{\circ}, 30^{\circ}$, and $45^{\circ}$, but is apparently higher in the model with the largest angle $\alpha=60^{\circ}$. This difference can be seen clearly in Fig. 5.25, which directly compares the cycle-averaged SWSSG between the four models. In particular, contrast the peak magnitude in the $60^{\circ}$ case (5.50), with that in the $45^{\circ}$ case $(3.48)$. 


\subsubsection{Results: RCA Waveform}

The flow patterns occurring in the RCA flow conditions are briefly summarized below. At the beginning of the systolic phase, a region of low-momentum flow is demonstrated just proximal to the heel. This low flow area is most extensive in the $15^{\circ}$ model and gradually disappears with increasing the angle. Also, a flow stagnation region is formed on the artery floor as a result of flow splitting. These features are persistent throughout almost the entire flow cycle. The flow skewing and secondary flow at the entrance of the distal artery are promoted during the diastolic deceleration phase, and is slightly more prominent in the $60^{\circ}$ model. This phenomenon, however, does not cause extremely low flow or flow stasis distal to the toe; the velocity of the flow there is only moderately reduced in all models.

The distribution patterns of cycle-averaged WSS in each model show great similarities to those in the LAD waveform case, whereas the OSI level in the RCA case is substantially lower. For all the models investigated, high SWSSG occurs during the diastolic phase when the flow rate is relatively higher. As the angle increases from $15^{\circ}$ to $45^{\circ}$, the change in the magnitudes of SWSSG is almost negligible (from 9.69 to 10.14). However, increasing the angle to $60^{\circ}$ causes a distinct increase in SWSSG (from 10.14 to 15.93$)$. Although the transient patterns of the spatial gradient for the RCA waveform are apparently different from those for the LAD waveform, the distribution and the level of cycle-averaged gradient are similar for the two waveforms. The peak magnitudes in cycle-averaged SWSSG from $15^{\circ}$ to $60^{\circ}$ are $2.87,3.10,3.39$ and 5.31, respectively. 

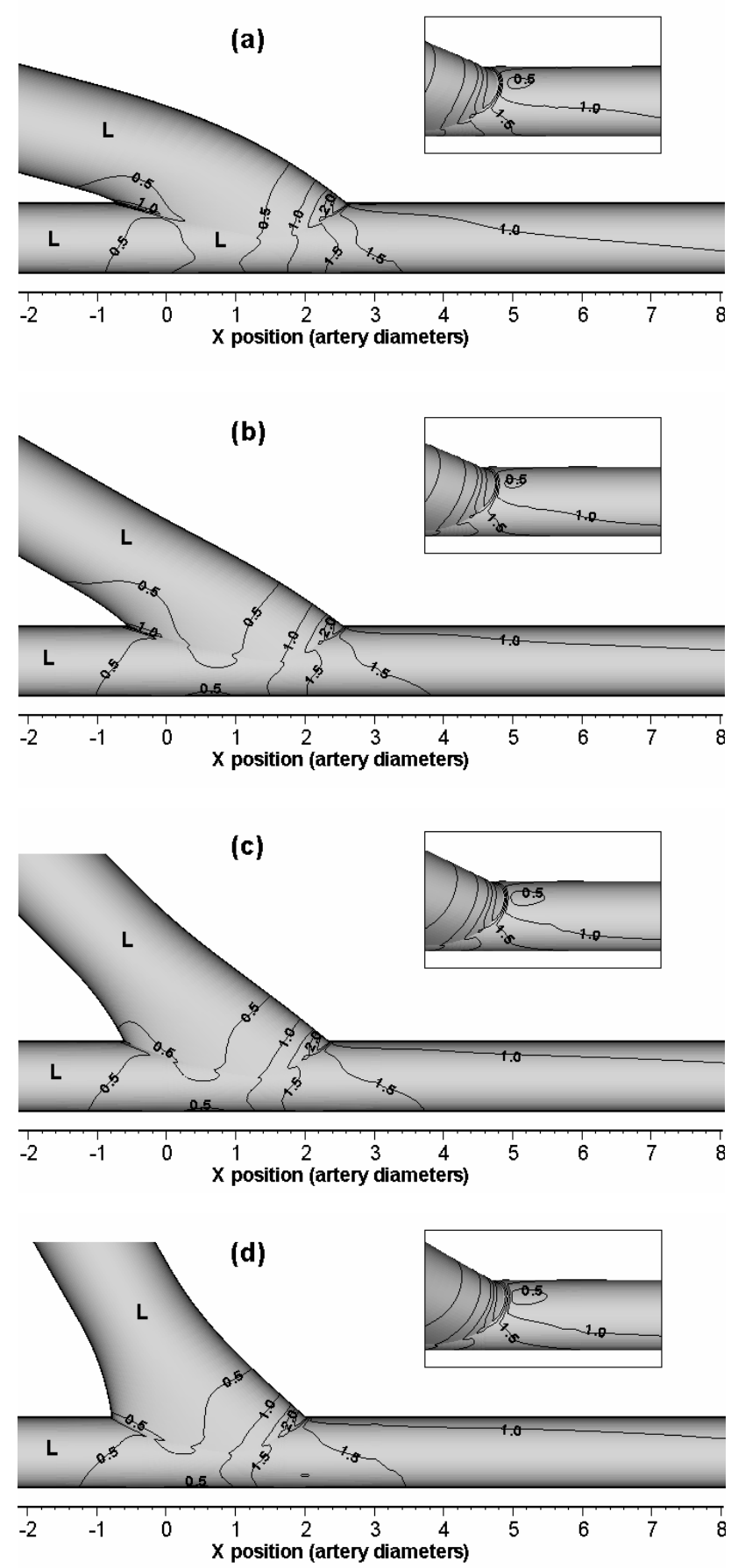

Fig. 5.23 Contours of cycle-averaged normalized WSS for the models with (a) $\alpha=15^{\circ}$, (b) $30^{\circ}$, (c) $45^{\circ}$, and (d) $60^{\circ}$. 

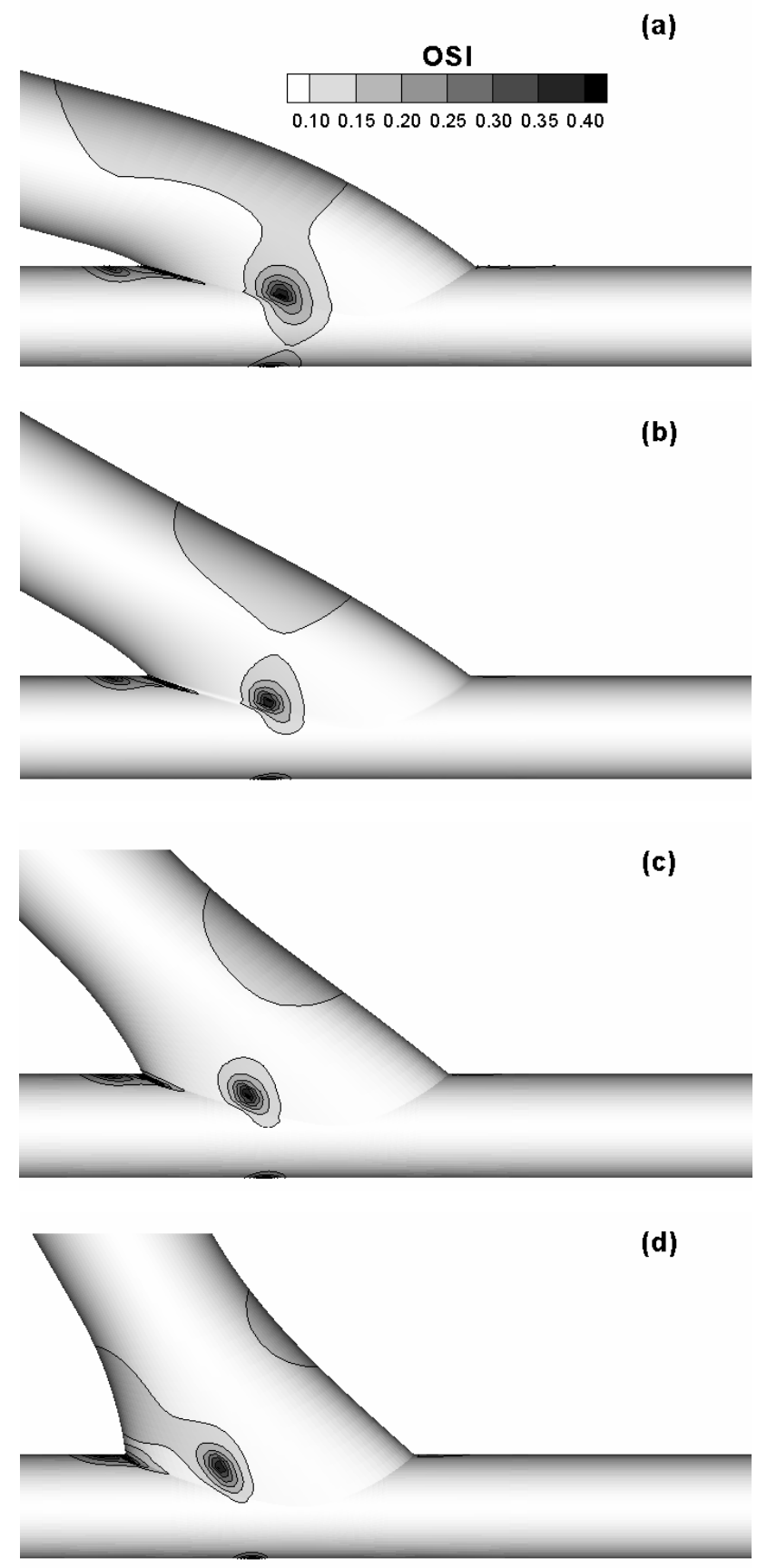

Fig. 5.24 OSI distributions for the models with (a) $\alpha=15^{\circ}$, (b) $30^{\circ}$, (c) $45^{\circ}$, and (d) $60^{\circ}$. 


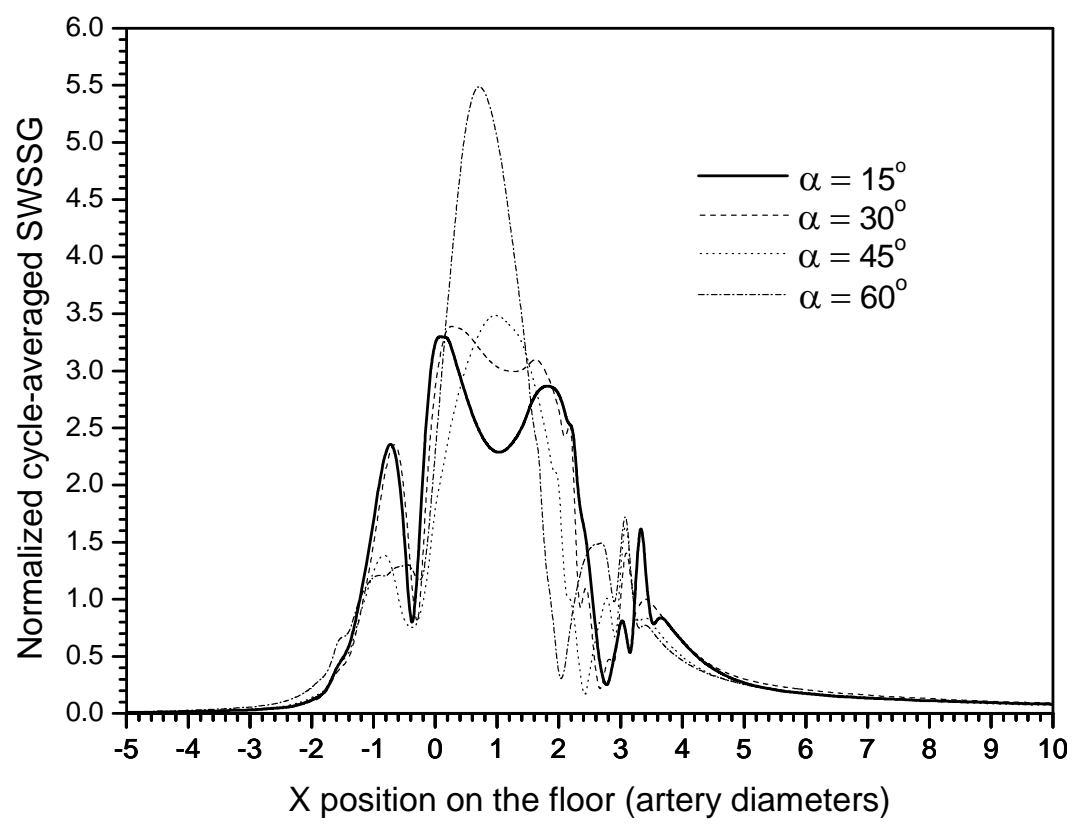

Fig. 5.25 Direct comparison of cycle-averaged SWSSG on the floor between the models with different angles.

\subsubsection{Discussion}

Varying the graft-artery angle apparently changes aspects of hemodynamics, although not as significantly as varying the diameter ratio does. Generally, a smaller angle is associated with a higher level of OSI in the anastomotic region, whereas a larger angle leads to higher SWSSG on the artery floor. It seems that the two medium angles of $30^{\circ}$ and $45^{\circ}$ represent a balance between the highest OSI and the highest SWSSG occurring in the $15^{\circ}$ and $60^{\circ}$ models, respectively. Fei et al. (1994) examined steady flow patterns at anastomoses of varying angles $\left(20 \sim 70^{\circ}\right)$ and a fixed diameter ratio (1:1) for a Reynolds number of 205 that was close to the mean value in the present study ( $\operatorname{Re}_{\text {mean }}$ $=230$ ). They found that the smallest angle produced the least amount of flow skewing and thus spatial gradients in shear stress in the floor region as compared to anastomoses with angles up to $70^{\circ}$, consistent with the results in the present study. However, no significant adverse effect was observed for small angles in their study. Hughes and How 
(1996) via an in vitro flow visualization technique investigated both steady and pulsatile sinusoidal flows in models with angles of 15,30 and $45^{\circ}$ and a 1:1 diameter ratio. They observed pronounced flow separation and flow reversal at the toe which extended several vessel diameters along the ceiling of the distal artery in the $30^{\circ}$ and $45^{\circ}$ models only, based on mean Reynolds numbers of 300 and 500. This phenomenon is not observable for all the models examined in the present study. The discrepancies between the above literatures and the present study are probably because they used a smaller graft-artery diameter ratio or a higher inflow velocity. These factors would increase the momentum of the graft flow, and thus accentuate downstream flow skewing towards the artery floor and encourage flow separation at the toe. Consequently, a smaller angle in those studies than in the present study would be required to compensate the adverse effects of flow skewing and separation. That is to say, the smaller the diameter ratio or the higher the flow velocity, the smaller angle should be adopted to inhibit flow separation and reduce high shear gradients.

The study of Jackson et al. (2001), although based on carotid artery anastomoses in rabbits, provided in vivo evidences for the findings in the present study that a smaller angle leads to higher levels of OSI and a larger angle leads to higher shear gradients. By assessing histological tissue responses to angles over $10^{\circ}, 45^{\circ}$, and $90^{\circ}$, they observed that the extremely small angle induces intimal proliferation, while the right angle results in wall atrophy with both cell loss and matrix degradation, showing maximal aneurysmal dilation in the native artery. The correlations between the in vivo observations and the present numerical results suggest mechanisms that high OSI stimulates or promotes intimal proliferation, whilst high shear gradients cause damage and regression to the endothelium of the vessel wall. Both intimal proliferation and 
endothelial damage are essential factors for ultimate intimal hyperplasia (Clowes et al., 1983).

This study suggests that the influence the graft-artery angle on anastomotic hemodynamics is important, but not as significant as that of the diameter ratio. The peak magnitude in cycle-averaged SWSSG, as the angle increases from $15^{\circ}$ to $60^{\circ}$, has increased by a factor of not more than 1 , whereas changing the diameter ratio from 1:1 to $1.5: 1$ results in an increase of a factor of $4 \sim 5$ in the same parameter. This is in line with the study of Moore et al. (1999), which suggested that the graft-artery diameter ratio should be classified as a primary geometric feature and the angle as secondary based on the flow conditions in the femoral anastomoses. Using a 20:80 proximal-distal outflow ratio, this study suggests that a graft-artery intersection angle of $30 \sim 45^{\circ}$ would improve hemodynamics.

\subsection{EFFECT OF MILLER CUFF GEOMETRY}

\subsubsection{Numerical Model}

Three Miller cuff models with various cuff configurations and one St Mary's boot model were investigated. The typical model geometries labeled with relevant terminology are shown in Figure 5.26. The generic features of the cuff models include a nearly $90^{\circ}$ attachment angle of the cylindrical cuff to the host artery, and an angle of approximately $10^{\circ}$ between the long axes of the graft and the artery. The native artery has a constant internal diameter of $d_{a}=3.3 \mathrm{~mm}$, and the diameter of the graft is chosen to be $1.5 d_{a}$. The cross-section of the graft distal end changes gradually from a round to an elliptical shape to meet with the cross-section of the cuff. For the high cuff model, the height of the cuff was specified to be $2.5 d_{a}$ and the length was $3.5 d_{a}$, yielding a 
length-height ratio (LHR) of about 1.4. The low cuff model was created by reducing the cuff height to $1.6 d_{a}$, resulting in an increased ratio of LHR $=2.2$. In the long cuff model, the cuff LHR was further increased to 3.3 by extending the cuff length to $5.2 d_{a}$. The St Mary's boot model was constructed by removing the rectangular graft toe of the long cuff model so that the original distal cuff wall is inclined at an approximate $40^{\circ}$ angle with the arteriotomy.

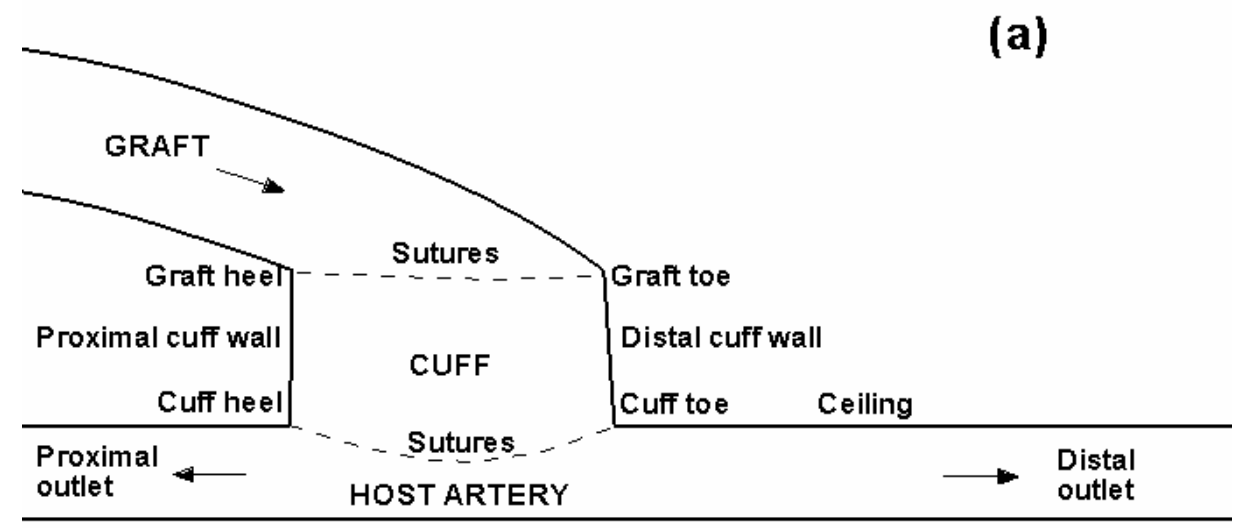

Floor

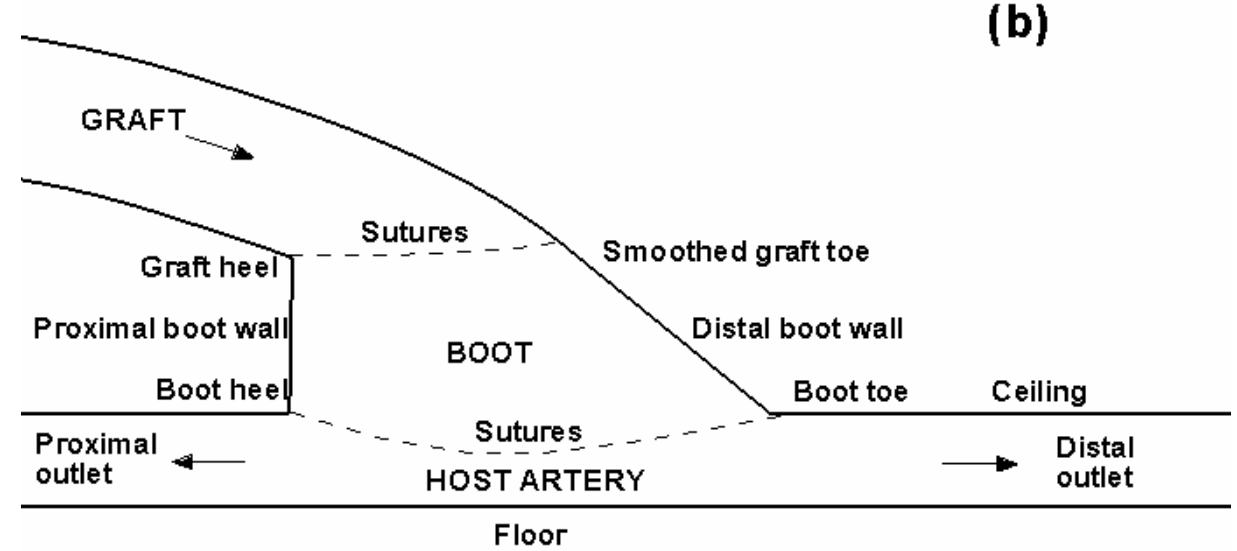

Fig. 5.26 Schematic picture of the model geometries: (a) Miller cuff, and (b) St. Mary's boot. The arrows indicate the main flow direction.

\subsubsection{Results}

Figure 5.27 to 5.30 show transient flow patterns in the LAD flow conditions for the high cuff, low cuff, long cuff and the boot models, respectively. The flow patterns in the same geometries using an infragenicular flow waveform are also presented in 
Appendices $\mathrm{A}$ in comparison with the LAD case. For the LAD waveform, most complex flow occurs during the negative flow phase of systole. At the early negative flow, all models demonstrate a large and coherent vortex in the middle of the cuff/boot. In the three cuff models, the fluid in the mainstream of the graft has higher kinetic energy and does not yet become retrograde until it approaches the distal cuff wall, dividing into two components, one rotating clockwise to form a large main vortex within the cuff, the other resulting in another area of flow separation and recirculation at the graft toe. The recirculation area at the graft toe is most prominent in the long cuff, and is completely eliminated in the boot model. There is no evidence of flow separation along the distal native artery for all the models investigated. At the late negative flow, the vortex within the cuff/boot and the recirculating flow around the graft toe in the cuff models remain almost unchanged. At the mid systole, the vortices in all models are replaced by an extensive low flow area (as indicated by short lengths of velocity vectors) in the vicinity of the cuff/boot proximal wall. Again, the long cuff model exhibits a large area of low flow at the graft toe. The flow in all models is less disturbed during the acceleration phase. No significant flow recirculation or vortex is recognizable except a flow separation region on the proximal part of the floor segment opposite to the cuff/boot where the flow splits into the proximal and distal components. Flow disturbances are again promoted in the diastolic deceleration phase. At the mid deceleration, an extensive low flow area, instead of a cohesive vortex, is demonstrated within the proximal half of the cuff/boot in all models. Again, extensive flow recirculation characterizes the graft toe of the long cuff model. In contrast, the flow at the smooth graft toe of the boot model is much streamlined with no flow disturbance recognizable. Furthermore, for all cuff models, the flow at the entrance of the distal artery segment, on negotiating the rectangular corner of the cuff toe to the distal artery, 
exhibits seriously skewed profiles towards the artery floor, vacating the superior corner immediately distal to the cuff toe filled with reverse flow and flow separation. This phenomenon is also accompanied by strong secondary cross-flows with a double helical structure in the distal artery of all cuff models as shown in the A-A view of the crosssection extracted at $0.5 d_{a}$ distal to the toe. The flow reattaches to the artery ceiling at a distance of nearly $1 d_{a}$ distal to the cuff toe. The flow reversal and separation in the distal artery persists till the end of the deceleration phase. For the boot model, flow separation does not arise in the native artery, and flow skewing and secondary flow are also found to be weaker.

For all the models investigated, the lowest cycle-averaged WSS characterizes the proximal half of the cuff/boot wall extending down to the artery floor (Fig. 5.31). Generally, the higher and broader the anastomotic region, the larger is the low-shear area. Low WSS is also found in the vicinity of the graft toe and along the distal artery ceiling just past the cuff toe in all cuff models. The boot model exhibits moderate WSS levels in the same regions.

In all models, the regions of high OSI resemble those with low WSS (Fig. 5.32). The long and high cuff models as well as the boot model show larger area of elevated OSI on the cuff/boot wall as compared to the low cuff model with a smaller anastomotic area. The long cuff model also shows the highest level of OSI at the graft toe. In addition, all cuff models exhibit elevated OSI along the distal artery ceiling immediately past the cuff toe. This area is slightly smaller in the long cuff model. In contrast, the corresponding regions in the boot model are almost devoid of elevated OSI. 

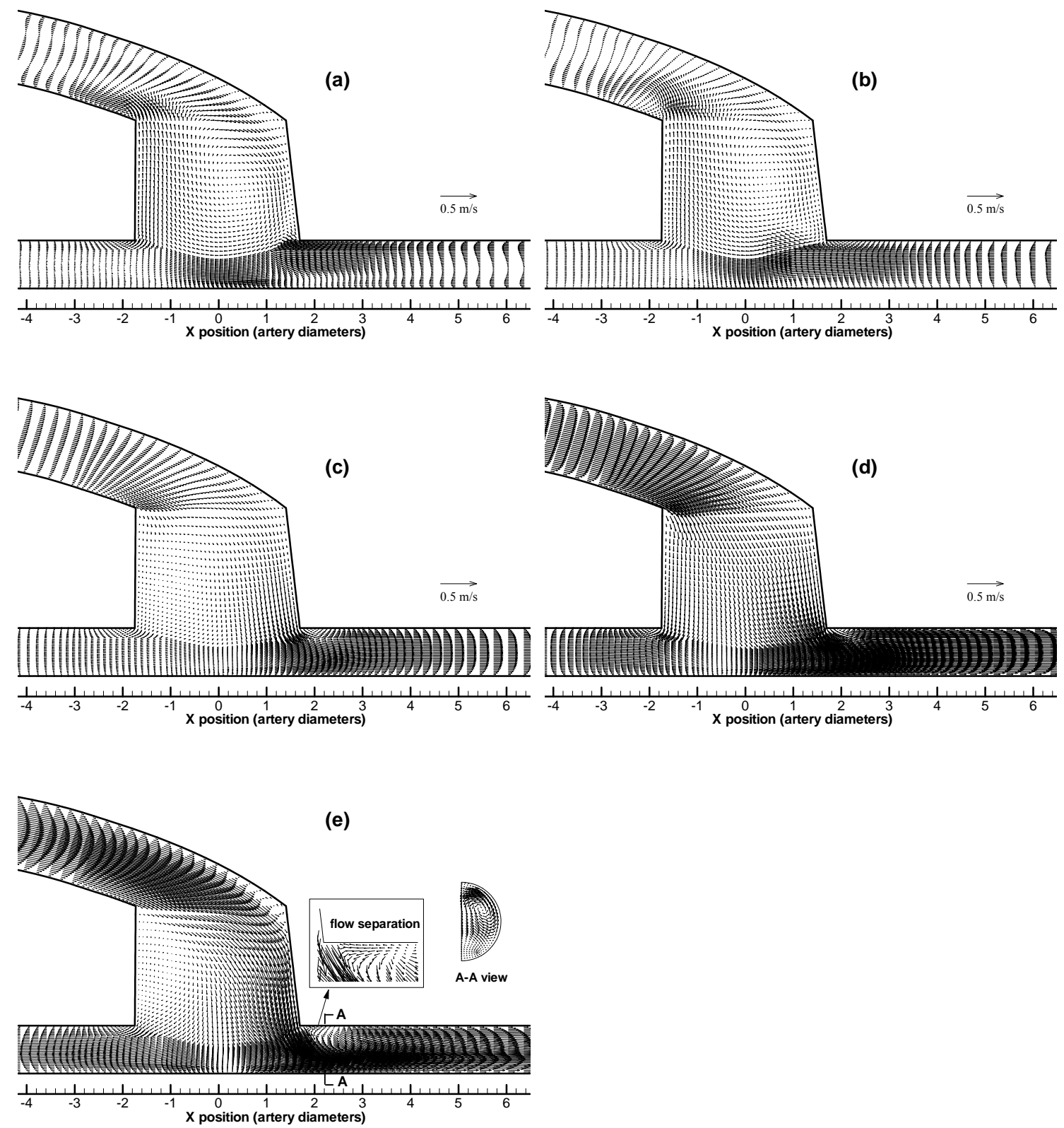

Fig. 5.27 Velocity vector plots in the symmetry plane for the high cuff model in the LAD flow conditions: (a) early negative flow, (b) late negative flow, (c) mid systole, (d) diastolic acceleration, and (e) diastolic deceleration. The small panel shows the secondary flow in the A-A cross-section, about $0.5 d_{a}$ distal to the cuff toe. 

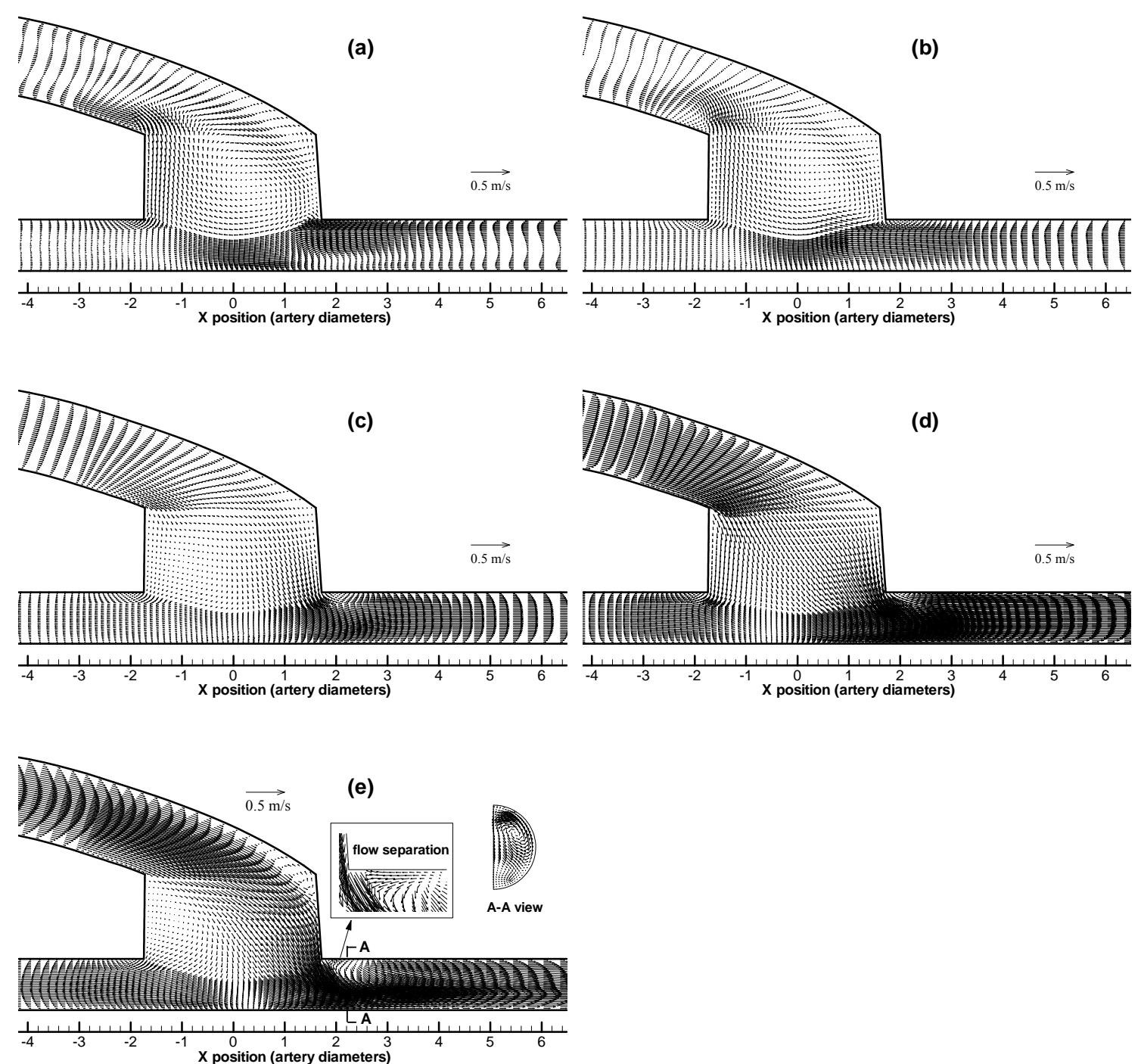

Fig. 5.28 Velocity vector plots in the symmetry plane for the low cuff model in the LAD flow conditions: (a) early negative flow, (b) late negative flow, (c) mid systole, (d) diastolic acceleration, and (e) diastolic deceleration. 

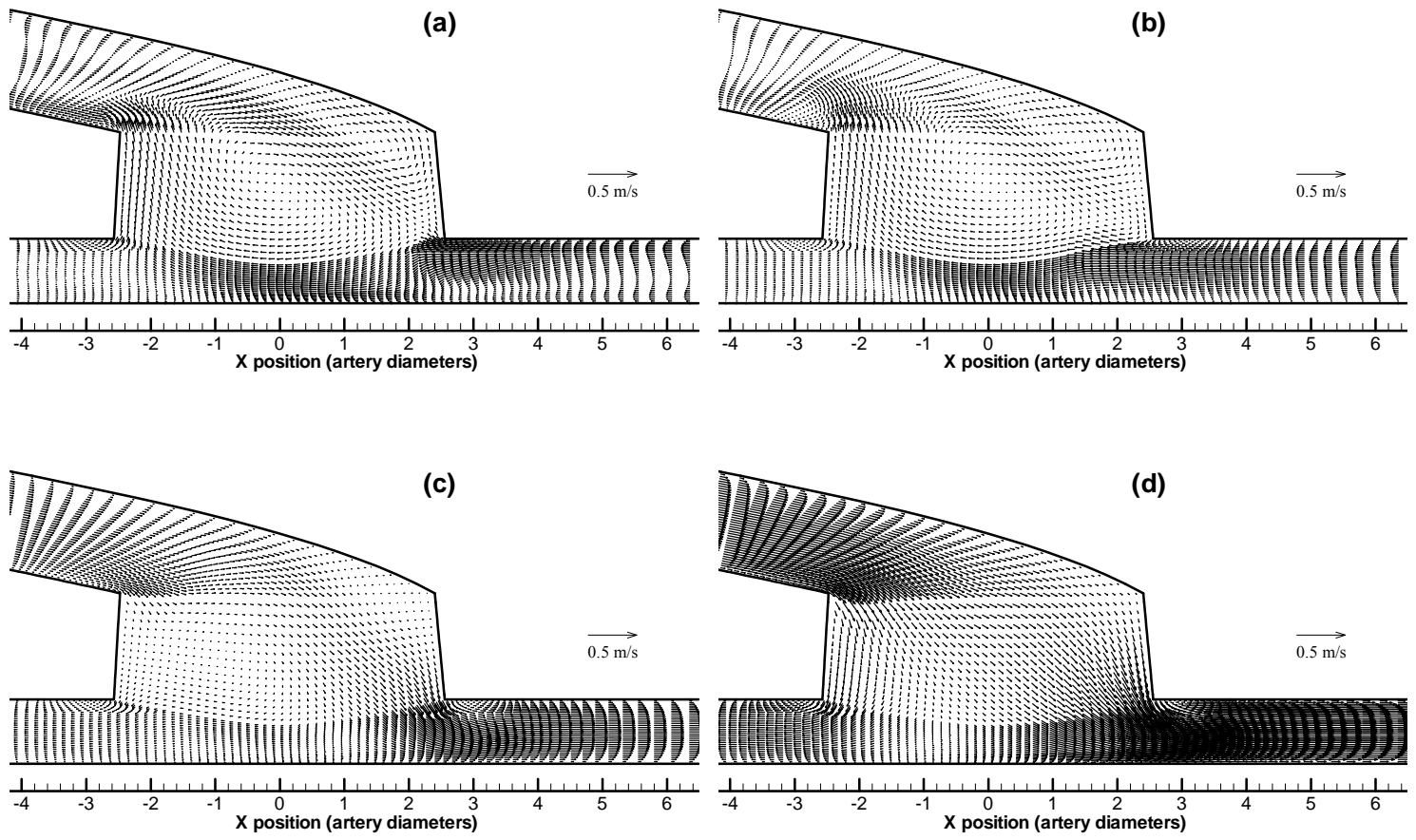

(d)

(e)

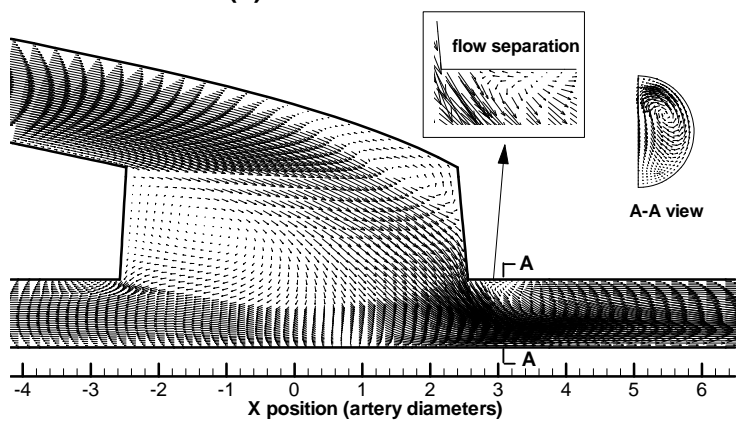

Fig. 5.29 Velocity vector plots in the symmetry plane for the long cuff model in the LAD flow conditions: (a) early negative flow, (b) late negative flow, (c) mid systole, (d) diastolic acceleration, and (e) diastolic deceleration. 

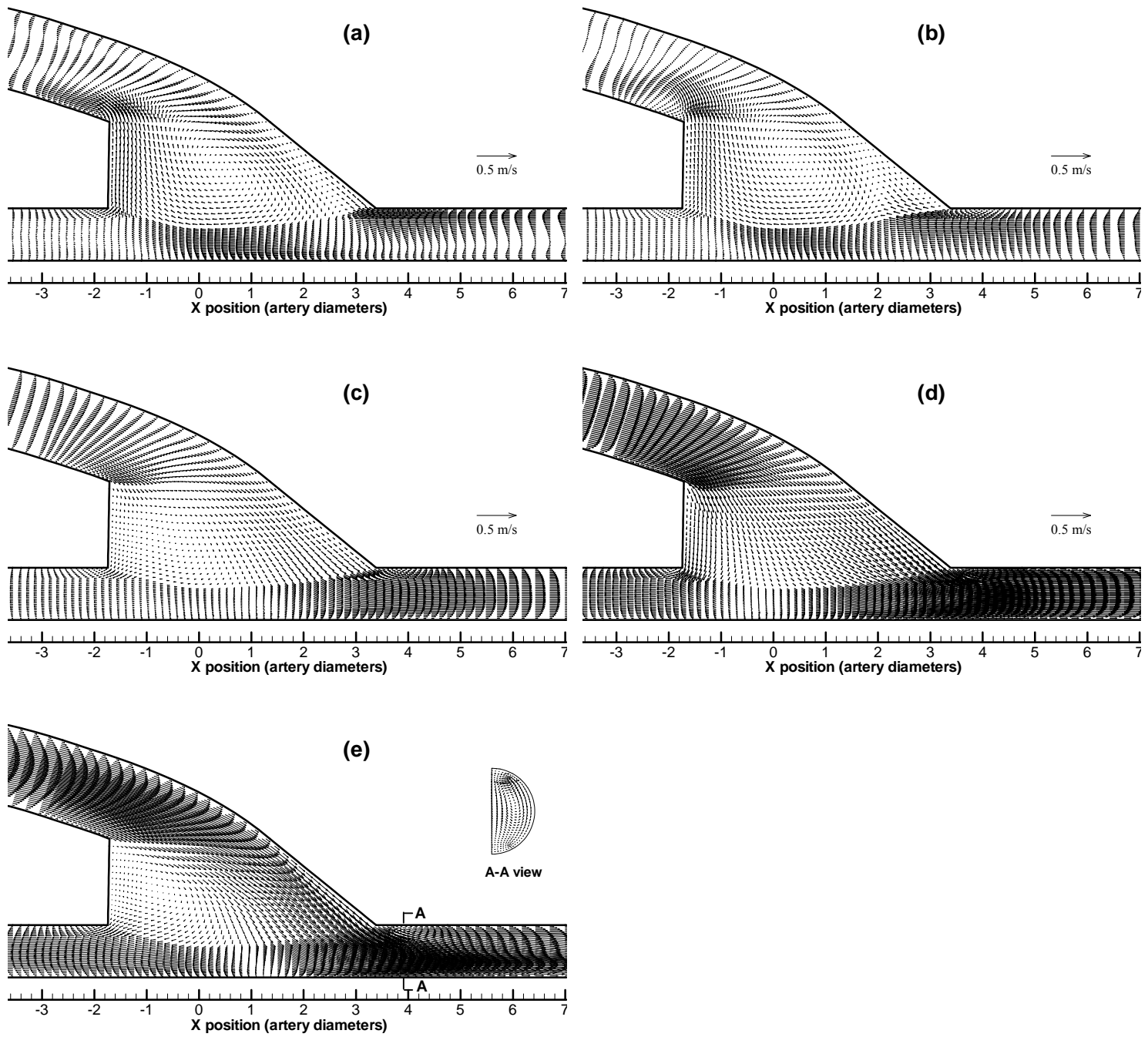

Fig. 5.30 Velocity vector plots in the symmetry plane for the St Mary's boot model in the LAD flow conditions: (a) early negative flow, (b) late negative flow, (c) mid systole, (d) diastolic acceleration, and (e) diastolic deceleration. 

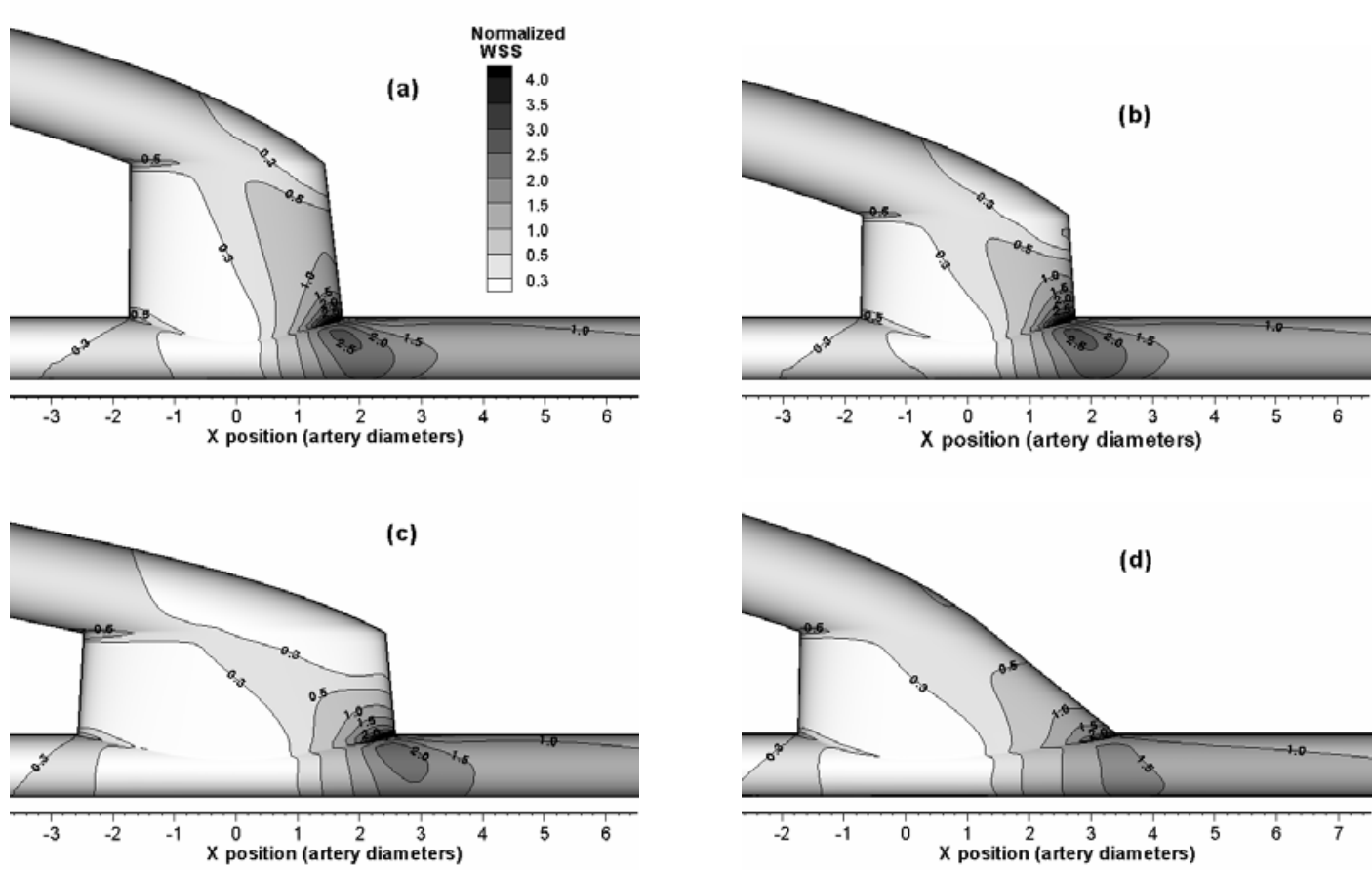

Fig. 5.31 Cycle-averaged WSS contours for the models with (a) high cuff, (b) low cuff, (c) long cuff, and (d) St Mary's boot.
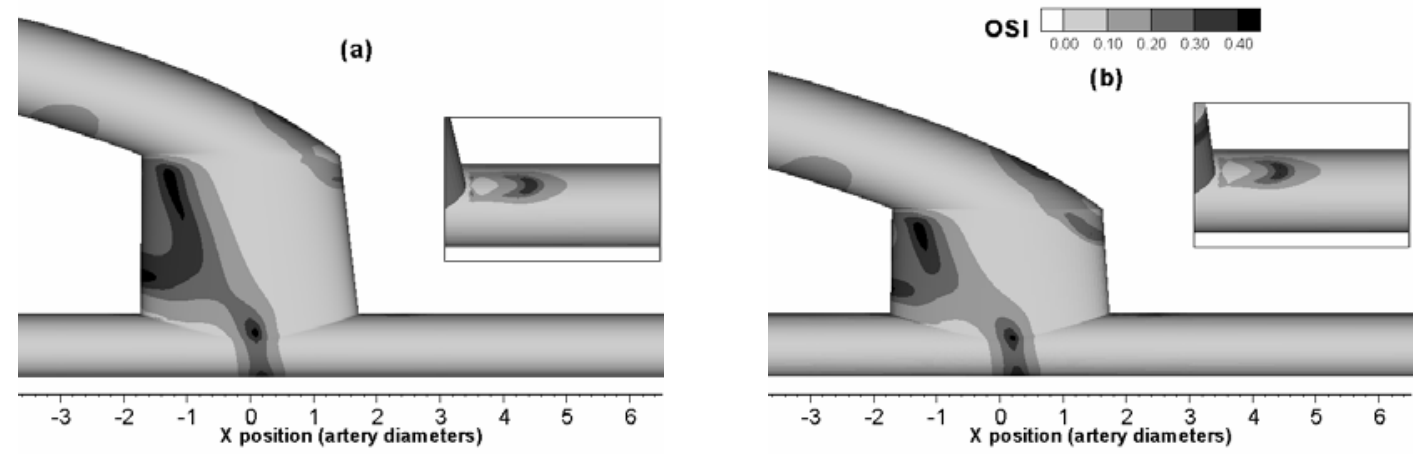

(c)
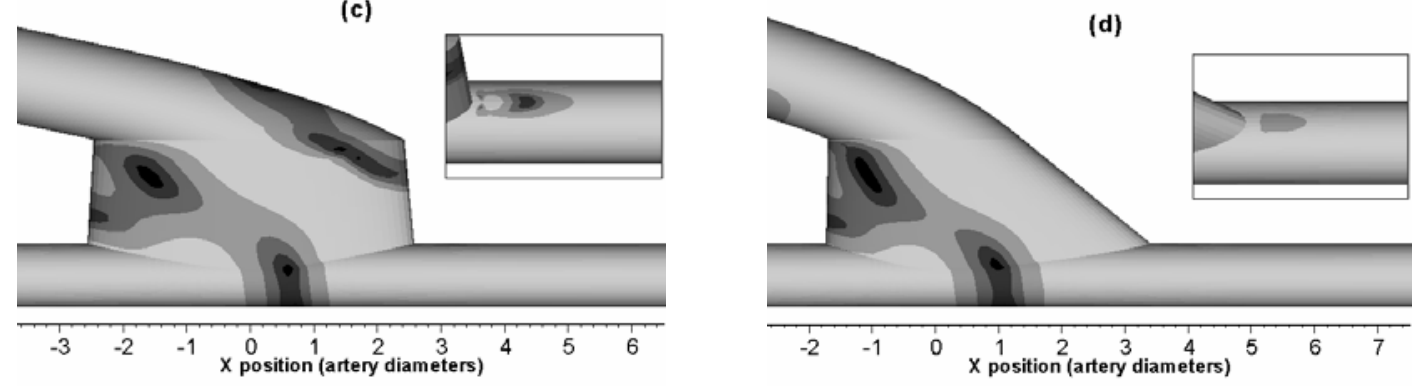

Fig. 5.32 OSI distributions for the models with (a) high cuff, (b) low cuff, (c) long cuff, and (d) St Mary's boot. 


\subsubsection{Discussion}

Superior patency rates have been reported for distal infragenicular reconstruction with a supplementary vein cuff between the prosthetic polytetrafluoroethylene (PTFE) graft and the host artery, compared with direct end-to-side anastomoses (Miller et al., 1984; Stonebridge et al., 1995; Morris et al., 1993). Examination of failed and failing prosthetic bypasses with and without a vein cuff revealed that the extent of $\mathrm{IH}$ is not reduced in cuff-supplemented anastomoses as compared to conventional anastomoses, but the greatest $\mathrm{IH}$ is shifted away from the cuff-artery interface to the more capacious upstream area around the graft-cuff interface (Tyrrell and Wolfe, 1997; Kissin et al., 2000; Trubel et al., 2004). While the biological and mechanical characteristics of the vein may constitute part of the reason for the favorable redistribution of $\mathrm{IH}$, a host of researchers believe that it is improved anastomotic hemodynamics associated with the unique cuff geometry that plays a more significant role in mitigating restenosis (Da Silva et al., 1997; How et al., 2000; Harris and How, 1999). In view of these studies, the cuffed anastomosis engenders a persistent and cohesive unidirectional vortex, which may produce a continuous wash-out effect, reduce area of low flow and flow separation along the native artery, and alter the distribution of wall shear stress and thus $\mathrm{IH}$ in a favorable manner. The goal of this study is to determine whether a hemodynamic advantage is associated with the unique geometry of the cuff anastomosis in the distal coronary anastomoses.

Although the cuffed procedure has improved the overall patency rates for below-knee bypasses, the randomized clinical results have been variable. A retrospective study reported poor results with the vein cuff: infragenual bypasses with a venous cuff had a one-year patency of $40 \%$, whereas in direct anastomoses this was $61 \%$ (Brumbe et al., 
1992). It is implicated that the inconsistent clinical results may be due to the cuff configuration being widely varied among patients (Fisher et al., 2001). Construction of the cuff involves cutting off a vein segment, incising the vein lengthwise, and stitching it so that the axis of the vein is aligned with the arteriotomy (Miller et al., 1984). Therefore, the height of the cuff is usually equal to the diameter of the harvested vein, whereas the cuff length is the length of the vein segment which is left primarily at the discretion of the operating surgeon. Fisher et al. (2001) investigated the influence of the cuff configuration on transient flow fields through in vitro flow visualization, and revealed that the vortical flow patterns within the cuff is profoundly altered by the cuff configuration, and this alteration may affect the graft performance to such a degree that they would change the conclusion on whether a cuff-supplemented anastomosis is beneficial. Therefore, this study considered three cuffs with widely-varied dimensions to ensure that the conclusion reached would not be biased by the use of an improper cuff configuration.

The results based on the infragenicular flow conditions show that the stability and cohesiveness of the vortex in the cuff is strongly affected by the cuff configuration, specifically the cuff LHR. Only the high cuff engenders a persistent and cohesive vortex, lasting from the beginning of the systolic deceleration to the end of the flow cycle. The vortex in the low cuff is less cohesive with no clear core of rotation during the systolic deceleration, and during early diastole it is replaced by a large area of flow stasis around the distal cuff wall. The flow within the long cuff is even more complex exhibiting highly irregular behavior during the systolic deceleration. Similar findings are reported by Fisher et al. (2001) in their in vitro study using similar infragenicular flow conditions. In the LAD coronary flow conditions in the present study, a cohesive vortex in the cuff is formed during the negative flow phase in each model, but it is a 
transient phenomenon with a duration of less than $1 / 3$ cycle, in contrast to $3 / 4$ cycle using the infragenicular waveform. During the long diastolic deceleration, the vortex becomes very weak, occupying a small area around the proximal cuff wall. These vortical flow patterns are similar in all cuff models. The more robust vortex produced in the infragenicular flow conditions may be ascribed to the higher deceleration rate of the infragenicular waveform, which tends to promote the vortex formation as compared to the mild deceleration of the LAD waveform. It is therefore suggested that the speculated wash-out effect based on a persistently cohesive vortex cannot be achieved when the cuff, regardless of its configuration, is applied in the coronary region.

The area of flow separation and recirculation at the graft toe appears in all cuff models and is persistent over the majority of the flow cycle in both infragenicular and LAD flow conditions. The extent of the area is a strong function of the cuff configuration. The long cuff demonstrates a particularly large area of flow separation and recirculation at the graft toe, whilst the area is much smaller in the low cuff and smallest in the high cuff. It is known that regions of disturbed near-wall flow patterns are susceptible to the formation of pathological alterations. For infragenicular cuffed anastomoses, flow separation in the presence of prosthetic PTFE material leads to platelet adhesion and activation which release growth factors, causing vascular smooth muscle cells to proliferate (Ombrellaro et al., 1996). It is thus indicated that the reported prevalence of $\mathrm{IH}$ at the graft-cuff interface is largely induced by impaired hemodynamics. Trubel et al. (2004) supported this finding by comparing different techniques with supplementary vein patches or cuffs such as the Linton patch, Taylor patch and Miller cuff. At the interface between the PTFE graft and the vein cuff/patch, the Miller cuff type exhibits the greatest $\mathrm{IH}$ which is almost twice that in the Linton or Taylor patches. Since the three types of anastomoses use the same materials and differ only in geometric 
configuration, the greater prominence of IH in the Miller cuff should be mainly caused by impaired hemodynamics, i.e. flow separation and recirculation as suggested in the present study. In fact, careful examination of failed bypasses revealed that it is IH accumulated at the graft-cuff interface that leads to the ultimate occlusion (Tyrrel and Wolfe, 1997; Kissin et al., 2000; Trubel et al., 2003). This observation, plus the finding in this study that the extent of flow separation greatly differs between the high, low and long cuff models, has confirmed the speculation that for infragenicular reconstructions, the cuff configuration (specifically, the length-height ratio) potentially influence the effectiveness of a cuffed procedure, and the clinical benefit can only be achieved when the cuff configuration has been created "right".

The formation of a vortex in the cuff is suggested to reduce area of low flow and flow separation along the native artery and favorably alter distribution of WSS (How et al., 2000; Harris and How, 1999). In the present study using the LAD flow waveform, however, sustained flow separation and thus high OSI are observed on the ceiling of the native artery immediately distal to the toe in all cuff models. It is logical since the flow must turn sharply around the rectangular corner of the cuff toe, vacating the superior area which is filled with disturbed fluid. In addition, the low-momentum vortical flow produced by the LAD waveform causes the WSS level on the proximal cuff wall and almost the entire artery segment adjoining the cuff to be considerably low. Such conditions, i.e. flow separation, low WSS and high OSI, are known to develop intimal thickening. Considering the results on conventional end-to-side anastomoses (as shown in the previous sections in this chapter) which show no flow separation and much lower OSI levels along the narrow native artery, it is again suggested that the geometry of Miller cuff would compromise anastomotic hemodynamics in the LAD flow conditions. 
It is also shown that a St Mary's boot configuration is hemodynamically superior to the Miller cuff in that it not only retains the vortex structure, but it also removes flow disturbances at the graft toe and cuff toe. Based on the premise that geometric alteration and not the supplementation of venous material is important in the supposed beneficial effect of the boot design for infragenicular procedures, a prosthesis preshaped to resemble the boot configuration has been developed, and recent clinical results are encouraging (Panneton et al., 2004; Oderich et al., 2005). However, the advantage of this design in the coronary region is questioned. The weak and short-lived vortex in the coronary flow conditions is not expected to produce a wash-out effect but rather result in extensively low WSS and high OSI on the boot and native artery.

This study has demonstrated that for infragenicular reconstructions, suboptimal cuff performance can be caused by impaired hemodynamics as a result of inappropriate cuff configuration by inducing rapid accumulation of $\mathrm{IH}$ at the interface between the prosthetic graft and vein cuff. Specifically, it is the LHR of the cuff that plays a critical role and should be carefully constructed. The St Mary's boot configuration is hemodynamically superior to the Miller cuff and is expected to further enhance the graft patency rates. However, the extrapolation of the Miller cuff geometry to the coronary region is not recommended as it would worsen hemodynamics in the native artery in the coronary flow conditions. 


\subsection{OPTIMAL DESIGN OF ANASTOMOTIC GEOMETRY}

\subsubsection{Numerical Model}

The study in the previous section shows that smoother transitions at the graft and cuff toes streamline the flow to the host artery and have considerably improved local hemodynamics at the anastomotic region. To optimize anastomotic geometry, a main step was to create smooth graft-artery transitions. In this study, four tentative models with different transitional curvature were constructed, giving variable aspect ratios at the toe ( $\lambda=R / a$, where $R$ is the radius of curvature and $a$ is the host artery radius) of 4 , 6, 8, and 10, respectively (Fig. 5.33a). Based on the results in Section 5.3 and 5.4, a graft-artery diameter ratio of $1.5: 1$ and an angle of $45^{\circ}$ were used for the design. Comparisons were then performed between the optimized, conventional, Miller cuff and St. Mary's boot geometries.

The stagnation region on the artery floor is notorious for disease development. Minimizing area of this region may be a means of reducing thickening on the floor. Therefore, the subsequent step of optimizing anastomotic geometry considers introducing a floor indentation to replace the stagnation region (Fig. 5.33b). The model with transitions properly smoothed in the last step was used to produce another two models with indentations of 0.25 and $0.5 \mathrm{~mm}$ on the floor, respectively. The location and shape of the indentation were determined according to the cycle-averaged WSS distributions for the model with flat floor so that the area with extremely low WSS on the floor is replaced. 


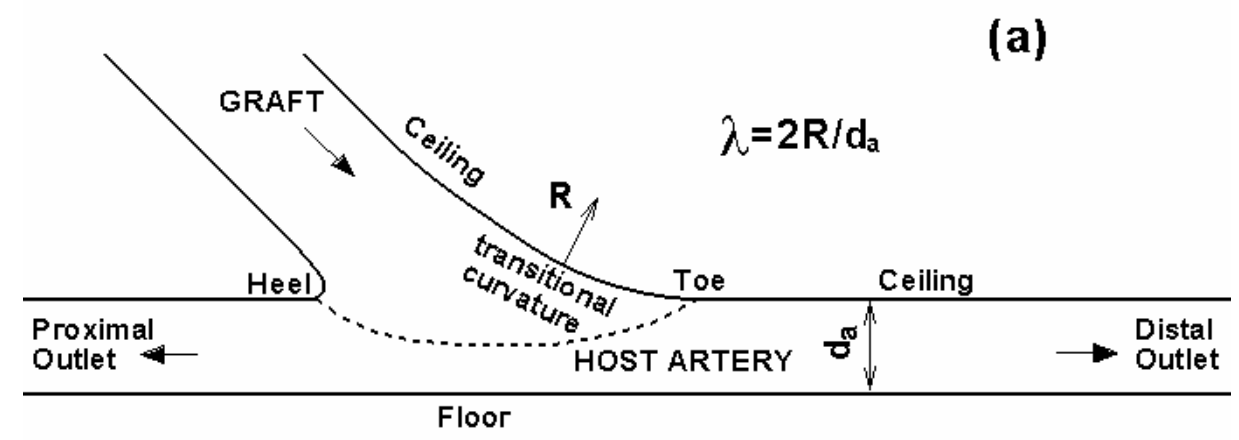

(b)

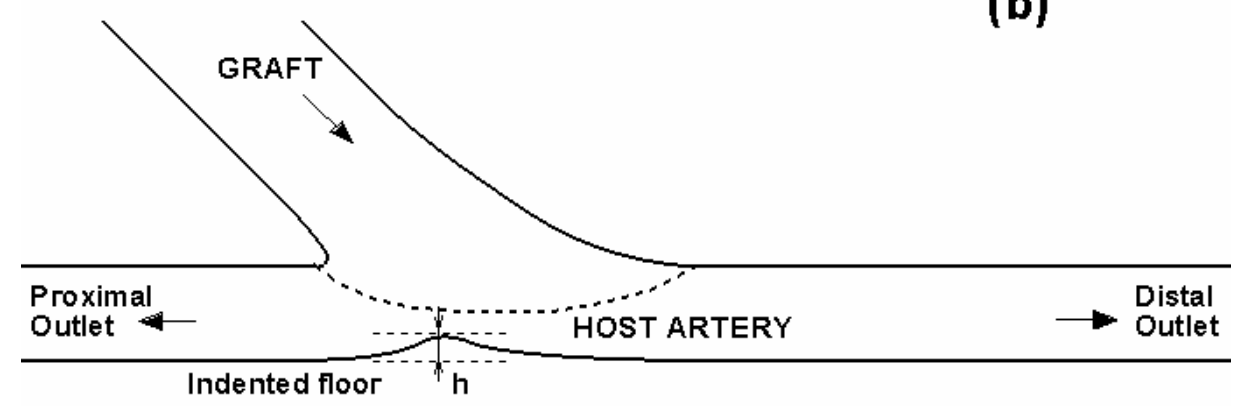

Fig. 5.33 Schematic picture of the optimized geometries with (a) flat floor and (b) indented floor.

\subsubsection{Results: Effect of Transitional Curvature}

Flow characteristics are similar in the four models with variable transitional curvatures. Transient flow patterns for the model with $\lambda=8$ are summarized in Fig. 5.34 in comparison with the conventional geometry. At the early negative flow, distinct difference between the modified and conventional geometries can be observed in the vicinity of the toe and heel. The sharp transition in the conventional geometry causes the flow to turn abruptly through the toe and heel, whereas the flow negotiates smoothly in the modified geometry. This difference is magnified during the late negative flow. In the conventional geometry, the high-speed fluid from the host artery experiences a sudden decrease in velocity on entering the graft, and tends to continue a distance before changing the direction. Consequently, a large portion of fluid in the distal end of the graft is appreciably diverted from the wall, vacating the corners at the toe and heel 
that are filled with very low flow. In contrast, the smooth transition in the modified case ensures the flow to be gradually decelerated as it approaches the anastomotic region, remaining attached and following the wall contour. Flow disturbances during the mid systole and diastolic acceleration are generally low in all models. When the diastolic deceleration phase commences, stagnant flow and flow separation in the conventional case, particularly along the ceiling of the distal artery near the toe, are again promoted and sustained till the end of the cycle. This phenomenon is accompanied by flow skewing towards the floor and secondary cross-flows in the distal artery segment. The modified geometry has apparently improved the flow field in these aspects.

The overall distributions of cycle-averaged WSS, OSI and SWSSG in the four modified models also show similar patterns. However, quantitative examination reveals clear difference between the four models. As illustrated in Fig. 5.35, cycle-averaged SWSSG on the floor has the highest level in the model with $\lambda=4$, with the peak magnitude of 3.20 ). Increasing the aspect ratio to 6 and 8 apparently reduces the peak magnitude to 2.18 and 1.61 , respectively. Further increasing the ratio to 10 causes a minor reduction in the same quantity (from 1.61 to 1.40 ).

Distinct differences in the distributions of wall parameters are found between the modified and conventional geometries. In the conventional case, elevated OSI is present on the ceilings of the graft and the distal artery next to the toe, whereas in the modified case the OSI level on the hood is reduced and the entire distal artery segment is devoid of elevated OSI. Figure 5.36 compares the curves of cycle-averaged SWSSG on the floor between the modified, conventional, high Miller cuff as well as the St. Mary's boot geometries. The high Miller cuff and the conventional geometries give the highest and second highest gradients with the peak value of 4.71 and 3.39 , respectively. The 
corresponding values in the modified and the boot geometries are reduced by a factor of $2 \sim 3$, being 1.61 and 1.48 , respectively.

\subsubsection{Results: Effect of Floor Indentation}

Small but important differences in flow patterns and distributions of wall parameters exist between the models with and without floor indentation. The flow stagnation region in the artery floor is gradually reduced when the height of indentation increases from $\frac{1}{8} \mathrm{~d}_{\mathrm{a}}$ to $\frac{1}{4} \mathrm{~d}_{\mathrm{a}}$, as compared to the model with flat floor. The movement of the stagnation point over the diastolic phase is restricted when the floor is indented by $\frac{1}{4} d_{a}$, in contrast to the curved path in the flat-floor model. The suppressed stagnation point, in turn, causes the OSI on the indented floor to be appreciably diminished in both magnitude and extension (Fig. 5.37). In addition, the area of high SWSSG on the indented floor is much narrower than on the flat floor (Fig. 5.38). 
(a)
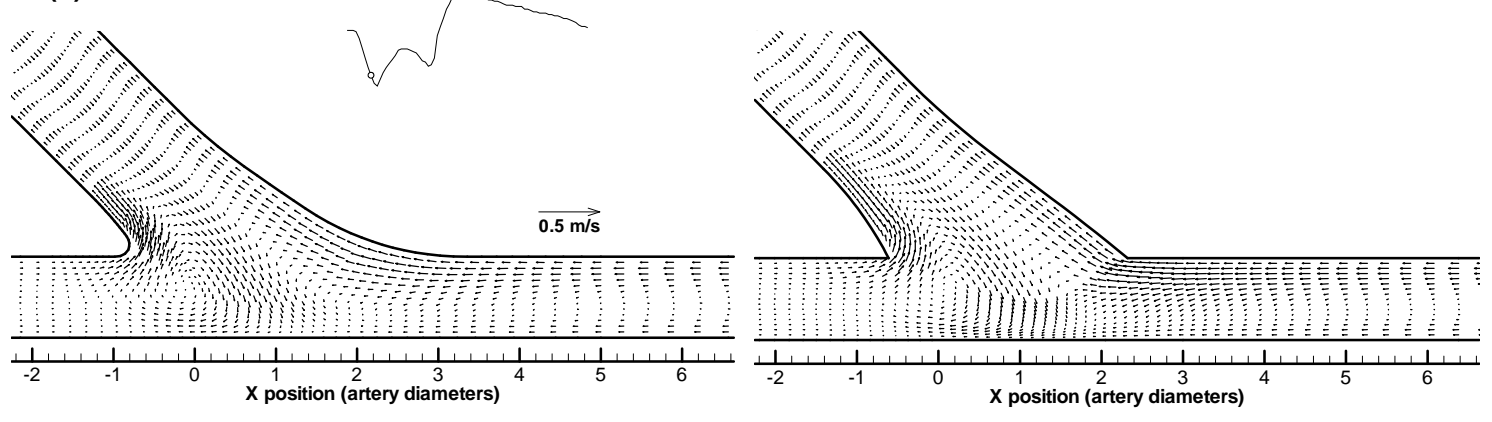

(b)
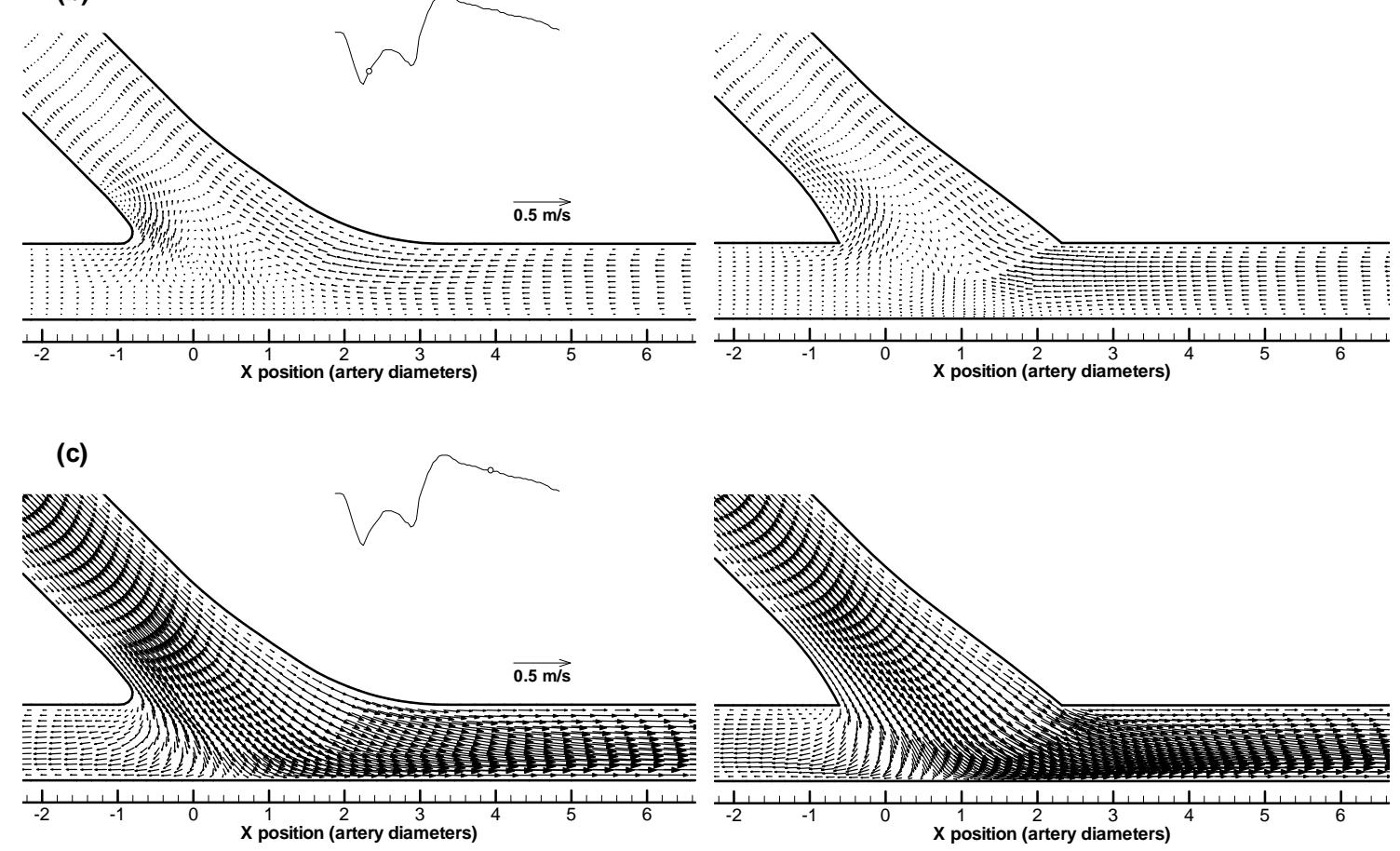

Fig. 5.34 Velocity vector plots in the symmetry plane for the optimized (left) and conventional geometries (right): (a) early negative flow, (b) late negative flow, and (c) diastolic deceleration. 


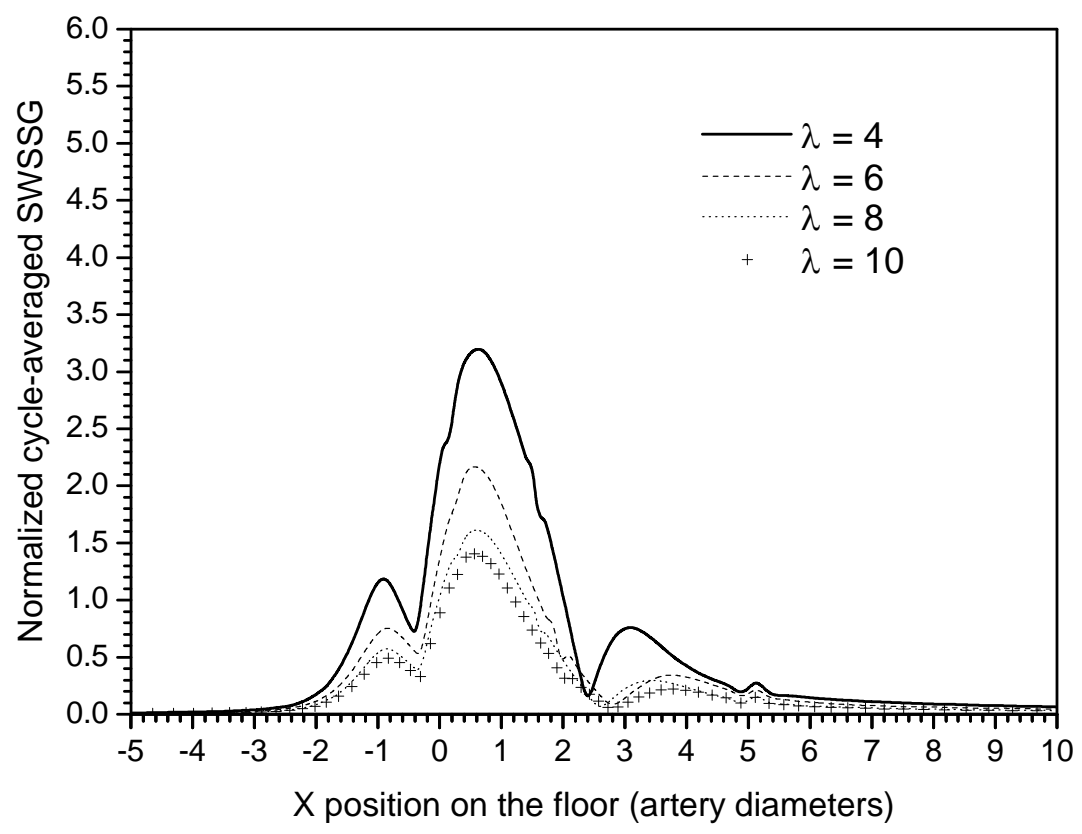

Fig. 5.35 Comparison of cycle-averaged SWSSG for the models with smoothed graftartery transitions. Here $\lambda$ is the aspect ratio at the toe.

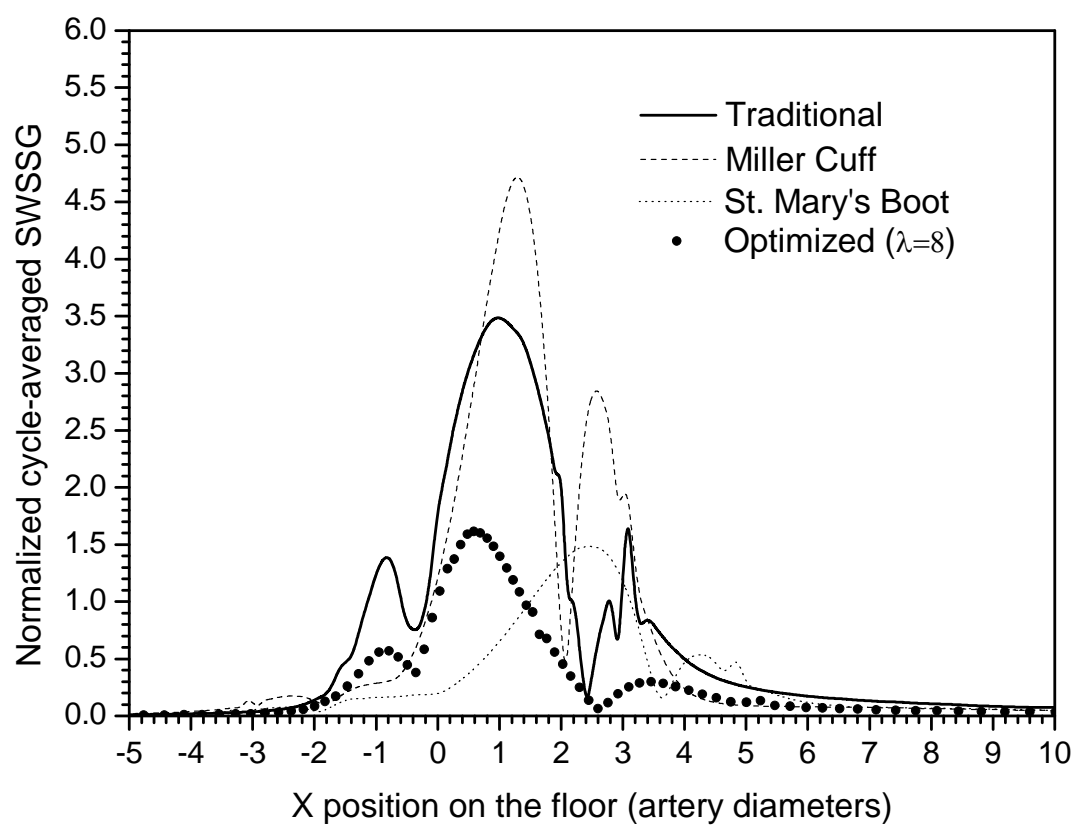

Fig. 5.36 Comparisons of cycle-averaged SWSSG between the conventional, high Miller cuff, St. Mary's boot and optimized geometries. 

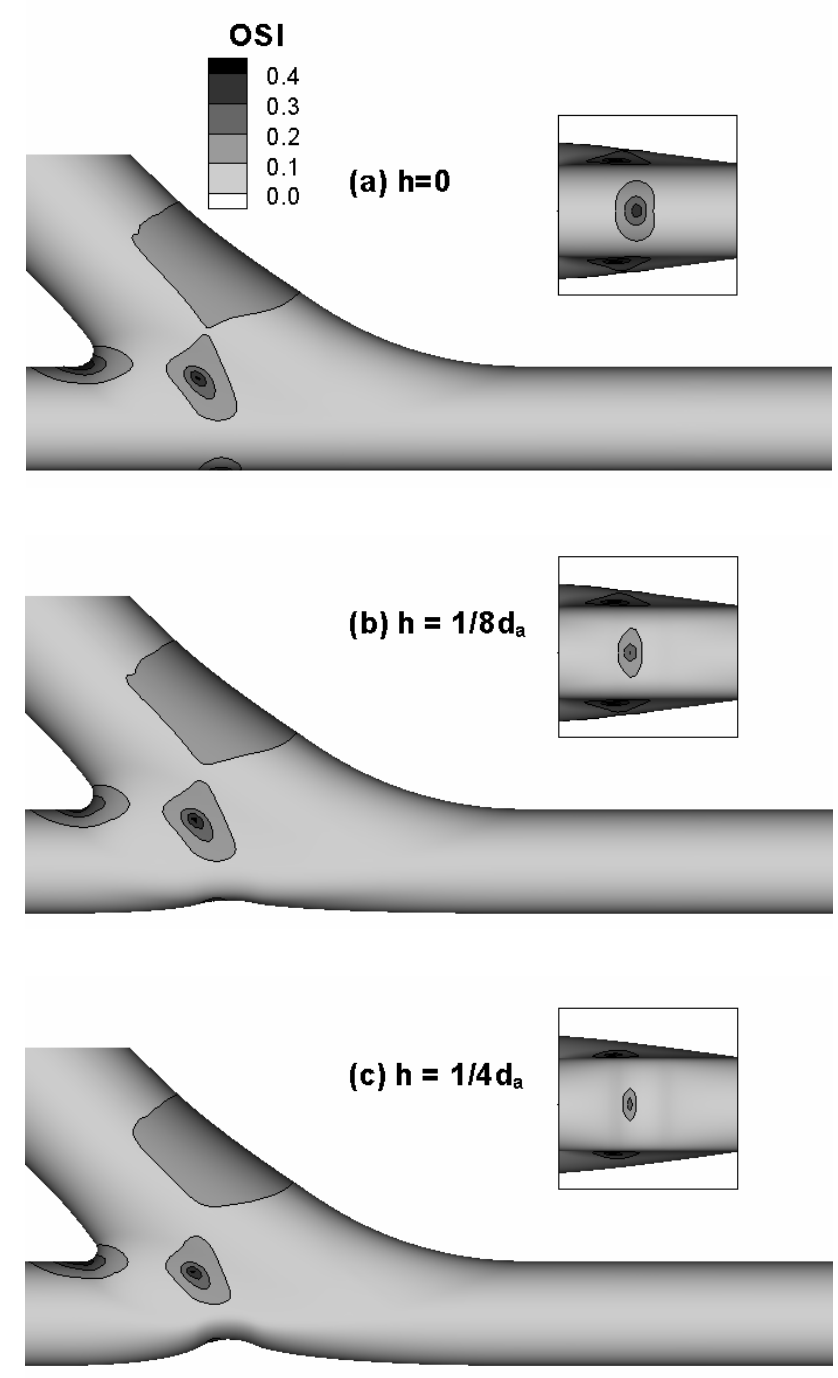

Fig. 5.37 OSI distributions for the models with and without floor indentation. The small panel shows the close-up view of the floor. 


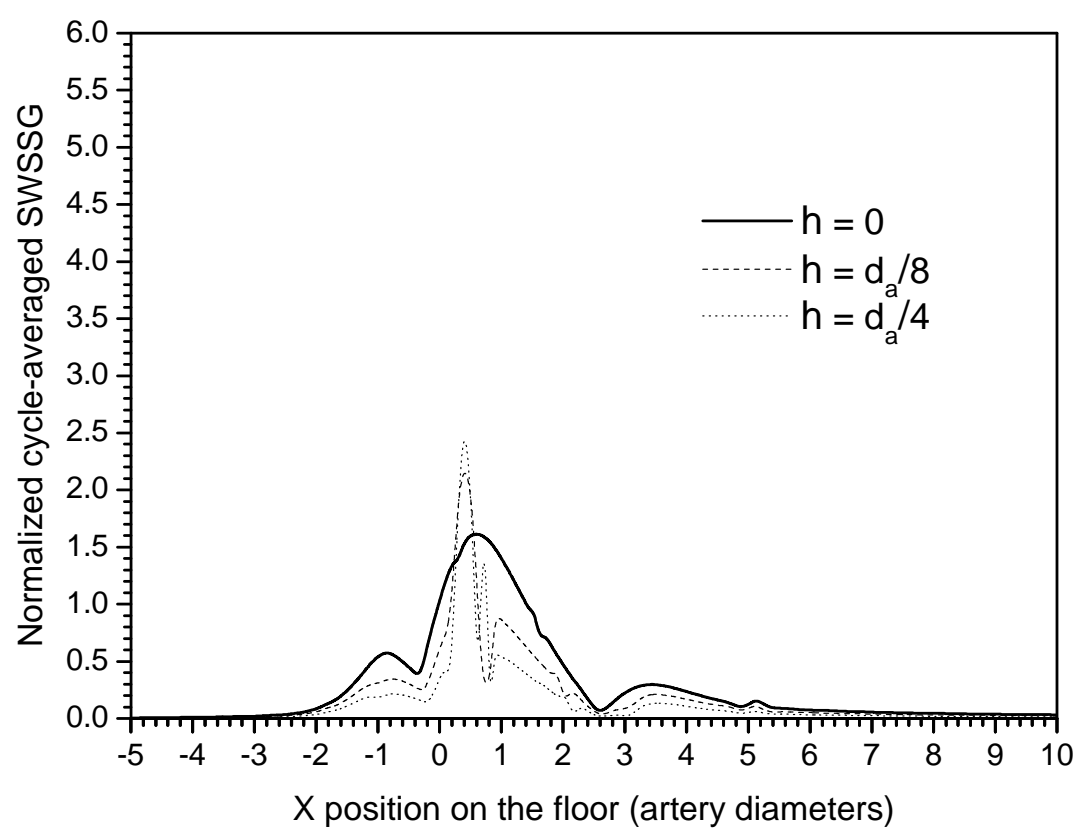

Fig. 5.38 Comparison of cycle-averaged SWSSG between the models with and without floor indentation. Here $h$ is the height of the floor indentation.

\subsubsection{Discussion and Summary}

It has been recognized that a proper transitional curvature between the graft and host artery has greatly improved the anastomotic flow, resulting in a more uniform WSS distribution and reduced SWSSG and OSI in the host artery. One may speculate that rapid restenosis is likely to be induced by sharp graft-artery transitions causing unfavorable hemodynamic environment and intimal thickening in the native artery. According to the numerical results, a larger aspect ratio at the toe transition generally gives rise to more streamlined flow, which is expected since the cross-sectional area is steadily becoming smaller from the graft to artery thereby avoiding abrupt changes to the average velocity of the blood. The aspect ratios of 8 and 10 are apparently superior to the ratios of 6 and 4 in terms of reduced SWSSG on the floor. Based on these observations, surgery should therefore be designed to obtain as smooth a toe transition as possible in light of fluid dynamics. 
Ojha (1994) via a photochromic tracer technique studied the effect of floor indentation on anastomotic flow and found that the placement of an elliptical plug on the floor of the distal anastomosis reduced the downstream motion of the stagnation point during the pulse cycle, which might prevent further endothelial deformation or injury. This is in line with the finding of the present study that the motion of the floor stagnation point is restricted during the diastolic phase when the indentation is present, and the area of the stagnant region and high OSI on the floor is also accordingly reduced. Smaller stagnation area corresponds to smaller area of elevated SWSSG. Therefore, it is suggested that surgically constructing an indentation on the artery floor is likely to mitigate intimal thickening. The indentation is created so as to replace the stagnation region. However, the location and size of the stagnation region can easily be changed due to changes in outflow ratio, and how flow in vivo distributes into proximal and distal outlet segments cannot be predetermined with current technologies. These issues make it difficult to apply the indented-floor geometry to practice, so this design is currently a mere speculation.

The comparison in SWSSG between the optimized, conventional, Miller cuff and St. Mary's boot geometries shows that the Miller cuff produces even higher SWSSG on the artery floor than the conventional geometry does, and the values in the optimized and boot geometries are substantially lower. As found in the previous section, the Miller cuff geometry is also associated with flow separation in the distal native artery as well as remarkably high OSI levels on the cuff wall and artery floor. It reconfirms the conclusion from the last section that local hemodynamics in coronary anastomoses is worsened when substituting the cuff geometry to the conventional one. The St. Mary's boot reduces SWSSG on the artery floor, provides more accommodating anastomotic 
area (i.e. less impedance) but at the same time potentially increases OSI levels as compared to the conventional case. The S-connector designed by Lei et al. (1997) for optimizing femoral anastomosis, is much similar to the St. Mary's boot: both have an acute graft-artery angle, enlarged anastomotic area, and smoothed toe transition. The Sconnector is thought to minimize cycle-averaged SWSSG (Lei et al., 1997); however, the OSI level on the artery floor is anticipated to be as high as that in the St. Mary's boot. Since whether high OSI or high SWSSG is more important in promoting floor intimal thickening is unknown, it is not definitive to say that either one of them, the boot, S-connector or conventional geometry, is more advantageous over the others. The optimal design of an end-to-side anastomosis should be the one in which both OSI and SWSSG are distributed in such a way that severe peak values of both parameters in the critical native artery can be avoided. For the geometry designed in the current study, both OSI and SWSSG on the arterial surfaces are much lower than for the conventional geometry. Therefore, it may represent a good solution for mitigating restenosis as well as protecting the native artery from further injury. Considering that constructing an anastomosis with geometry exactly following the optimal design data is not feasible during a surgery, a prefabricated synthetic connector with linings of endothelial cells is recommended. To achieve this, a "sleeve" technique is proposed and will be discussed in the next chapter. 


\section{CHAPTER 6}

\section{HEMODYNAMIC ASSESSMENT OF INTERPOSITION SLEEVE}

\subsection{INTRODUCTION}

In order to apply the optimal geometry in vivo, the use of a premanufactured biocompatible "sleeve" connecting the graft and recipient artery is recommended. A schematic description of this technique is shown in Fig. 6.1 in comparison with the conventional procedure. This sleeve technique not only takes advantage of the optimized geometry to achieve improved hemodynamics (especially in the native artery), but it is also preferable in many other ways. First, it can ease surgical manipulation (suture-less or minimal sutures) to reduce surgical trauma and operating time. Second, it can standardize the surgical procedure to ensure good quality and avoid technical error. The learning curve for the surgeons to be competent for the surgery would also be accordingly shortened. In addition, a sleeve can be properly designed to cover all the disease-prone regions, and therefore may act as a protector for the native artery. The present chapter will focus on assessing hemodynamic performances of the sleeve in terms of proximal-distal outflow ratio, graft-artery nonplanarity, and thickness of sleeve wall based on the optimized geometry designed in Chapter 5 .

\subsection{EFFECT OF PROXIMAL-DISTAL OUTFLOW RATIO}

\subsubsection{Numerical Model}

The flow division ratio in the proximal and distal outlet segments varies between individuals and locations, and may even vary during different phases of a cardiac cycle. Test on different outflow ratios would provide more information as regards the 
applicability and efficiency of the design. Five ratios, i.e. $Q_{g}: Q_{p}: Q_{d}=100: 0: 100$ (zero proximal flow), $Q_{g}: Q_{p}: Q_{d}=100: 10: 90$ (10\% proximal flow), $Q_{g}: Q_{p}: Q_{d}=100: 20: 80$ (standard case), $Q_{g}: Q_{p}: Q_{d}=100: 30: 70$ (30\% proximal flow), and $Q_{g}: Q_{p}: Q_{d}=$ 100:40:60 (40\% proximal flow), were examined (Fig. 6.2).
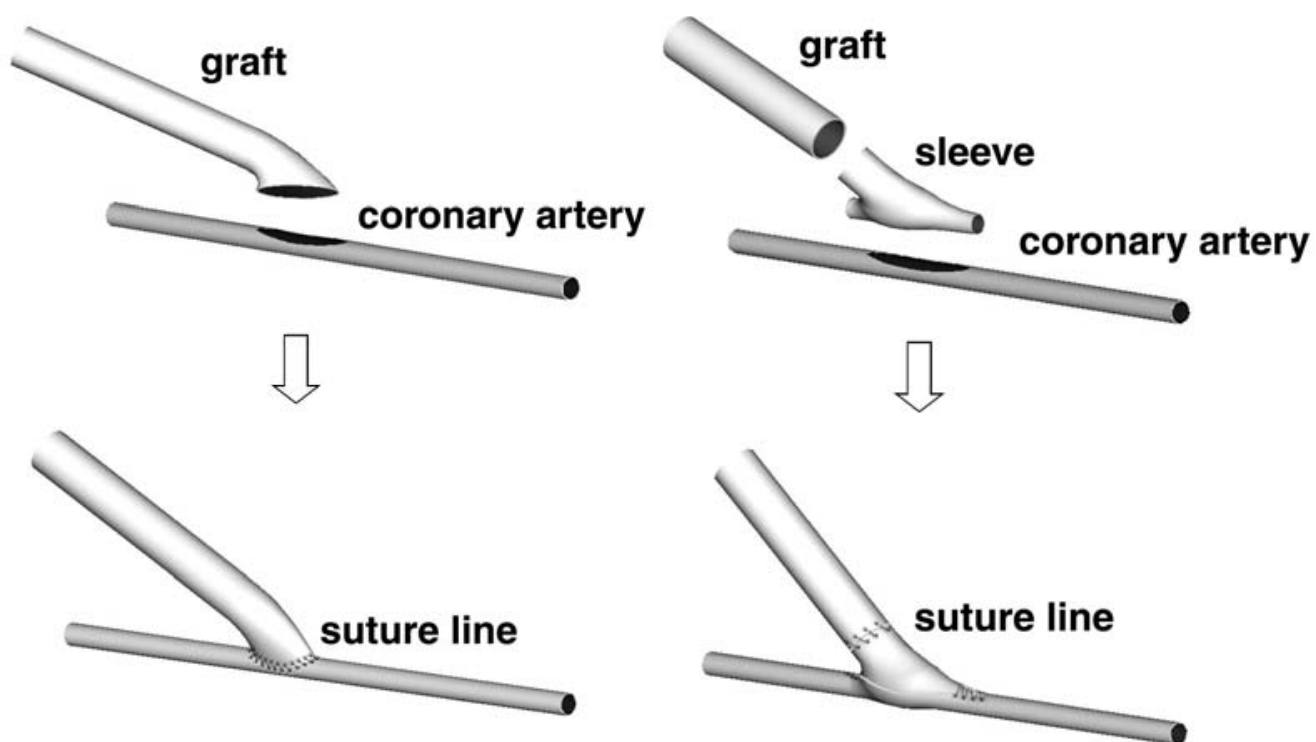

Fig. 6.1 Procedures for the conventional (left) and sleeve (right) techniques. In the conventional procedure, the graft is simply connected to the host artery; while in the sleeve procedure, a sleeve device is placed with one end inserted into the graft and the other two ends as well as the lower portion into the recipient artery.

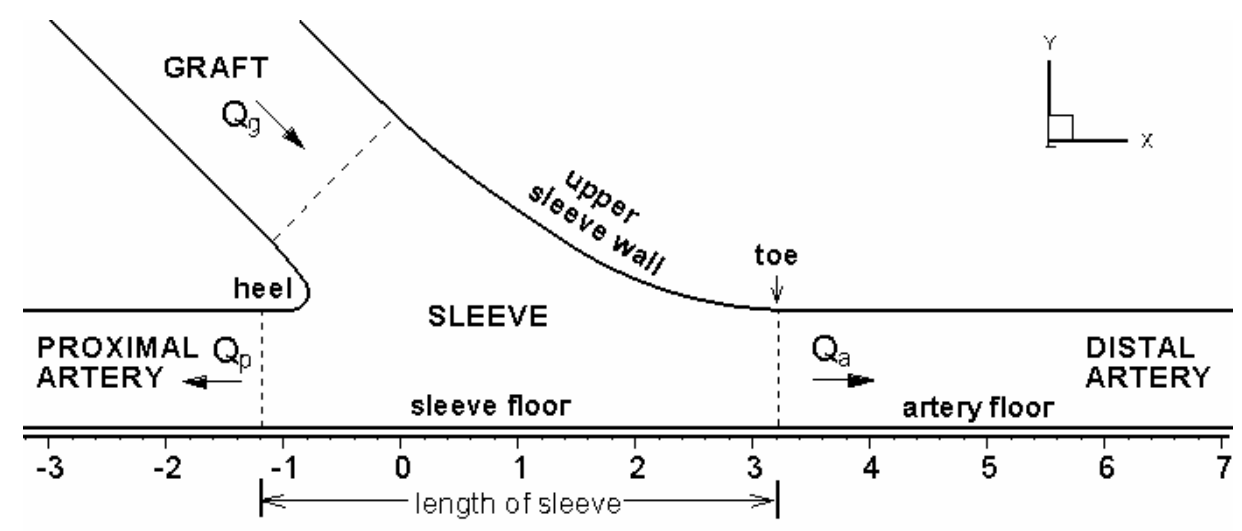

Fig. 6.2 Schematic picture of the sleeve model. The sleeve area is enclosed with the dashed lines. 


\subsubsection{Results}

There are distinct differences in the flow field for each case with different proximaldistal outflow ratios. Flow characteristics at four typical time steps for the two extreme cases (i.e. zero and 40\% proximal outflow) are shown in Fig. 6.3 and 6.4, respectively, in comparison to those in the standard case (Fig. 5.34). At the early negative flow, a region of flow recirculation in the proximal part of the sleeve is demonstrated in all the cases. Increasing the portion of the proximal outflow causes the recirculating flow to become weaker and shift towards the proximal outlet segment. In the case of zero proximal outflow, the retrograde flow along the sleeve floor gradually diminishes to zero in velocity as it approaches the position across the heel, and no flow is observed throughout the entire proximal artery segment. At the late negative flow, for all the cases investigated, the recirculating flow in the sleeve is attenuated and extensively low flow characterizes the sleeve floor. At the mid systolic flow, the flow returns to its primary direction and the area of flow recirculation is cleared in all the cases. For the zero proximal flow case, the low flow area around the sleeve proximal end is found to be more extensive. For the other cases, a flow stagnation region on the sleeve floor resulting from flow splitting is formed, and this region moves distally as increasing the portion of proximal outflow. During the diastolic deceleration phase, the flow field in all the cases becomes more disturbed. In the case of zero proximal outflow, a lowmomentum flow recirculation is again promoted in the proximal part of the sleeve as illustrated by the streamtraces, which extends from the heel to the middle position of the sleeve floor. Increasing the proximal outflow tends to reduce the size of the stagnation region on the floor, but on the other hand cause the flow velocity along the upper sleeve wall to be decreased. In the case of $40 \%$ proximal flow, for example, the flow stagnation area along the sleeve floor is negligible, whereas low flow is observed 
along the upper sleeve wall. All these flow features are sustained throughout the entire diastolic deceleration, which lasts more than half of the cardiac cycle.

Low WSS is hypothesized to induce thrombus formation and neointimal hyperplasia on synthetic surfaces (Schoephoerster et al., 1993; Ku and Zhu, 1993). An inverse, nonlinear relationship between the thickness of neointimal thickening in PTFE grafts and shear stress is shown in Fig. 6.5. It can be observed that the thickness increases rapidly when the shear stress is below 5 dynes $/ \mathrm{cm}^{2}$, and therefore this value will be used as the threshold in the present study. Figure 6.6 shows the WSS distributions for all the cases. Note that the area of low WSS $\left(<5\right.$ dynes $\left./ \mathrm{cm}^{2}\right)$ on the sleeve surface is smallest in the standard case. Both increasing and decreasing the proximal outflow tend to enlarge this area. This area of low shear is not prominent for all the cases of $10 \% \sim 40 \%$ proximal portion. In the case of zero proximal outflow, however, the proximal outlet segment of the sleeve as well as the entire proximal artery is covered by low shear. Increasing the proximal outflow gradually shifts low WSS from the proximal part of the model upward to the upper sleeve wall. 
(a)

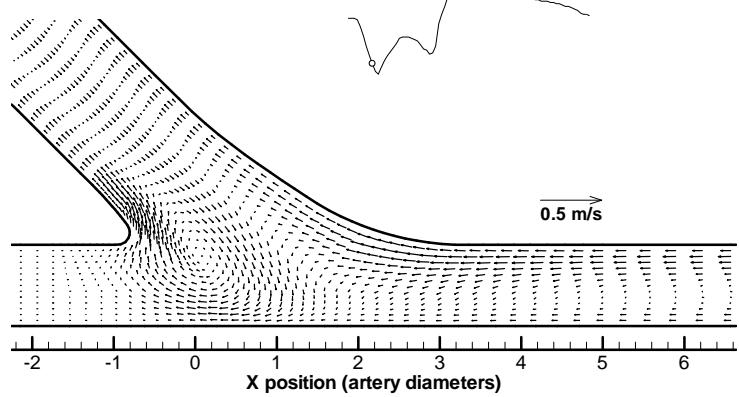

(c)

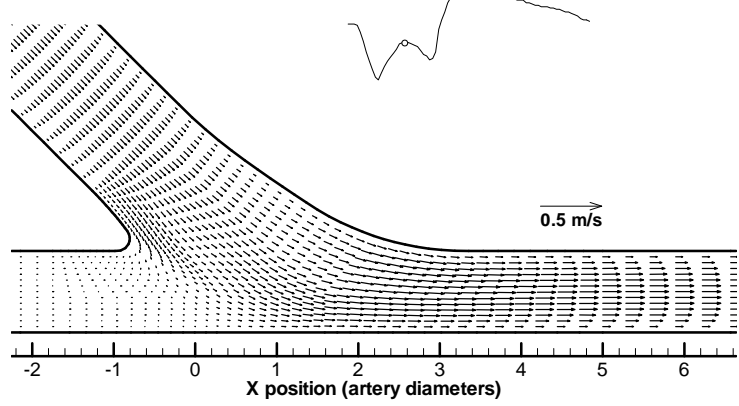

(b)

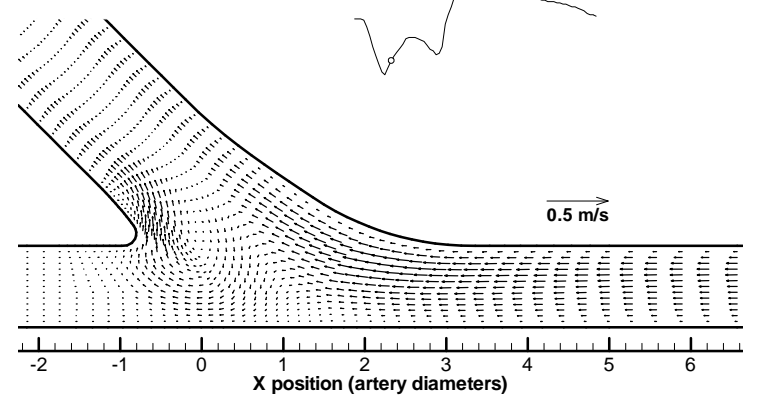

(d)

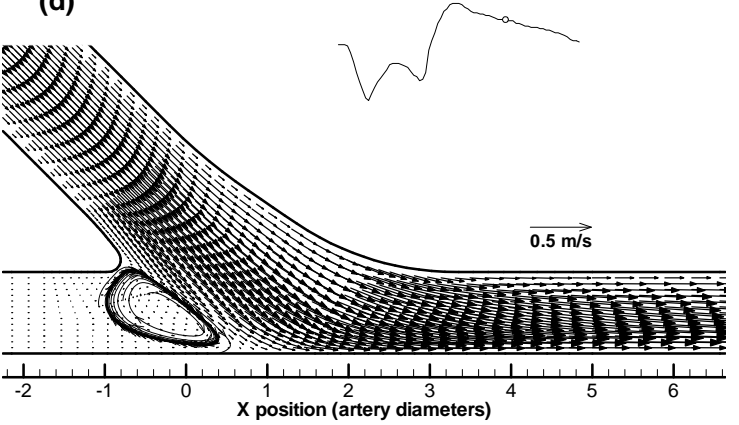

Fig. 6.3 Velocity vector plots for the sleeve model with zero proximal outflow: (a) early negative flow, (b) late negative flow, (c) mid systole, and (d) diastolic deceleration.

(a)

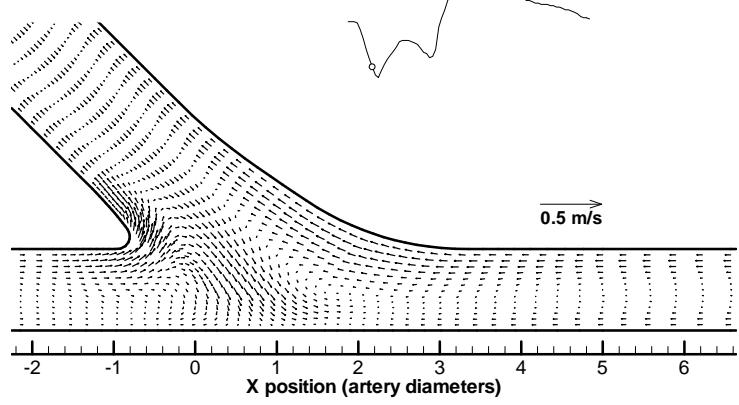

(c)

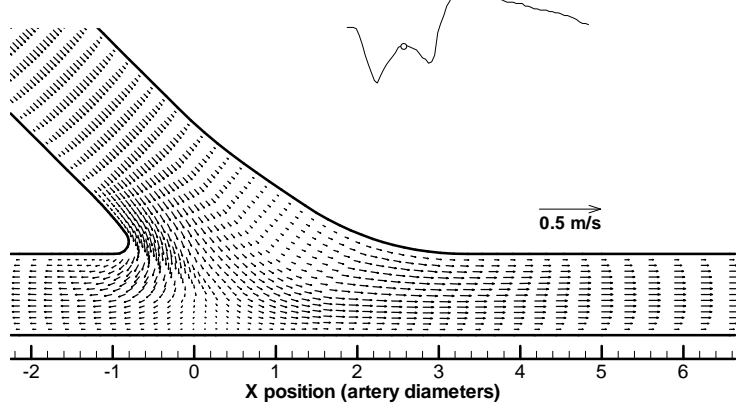

(b)

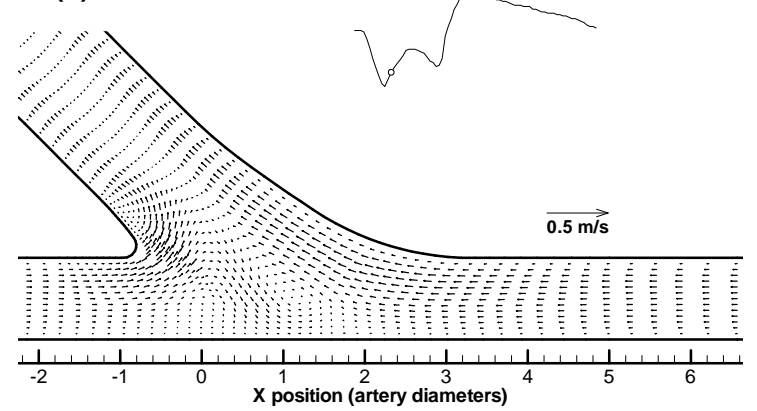

(d)

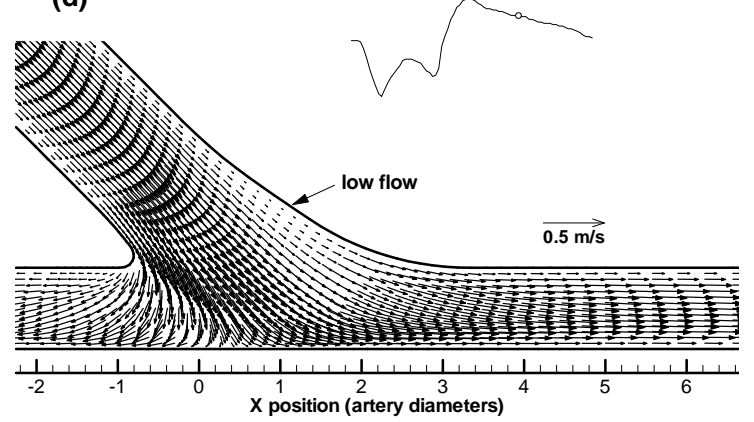

Fig. 6.4 Velocity vector plots for the sleeve model with $40 \%$ proximal outflow: (a) early negative flow, (b) late negative flow, (c) mid systole, and (d) diastolic deceleration. 


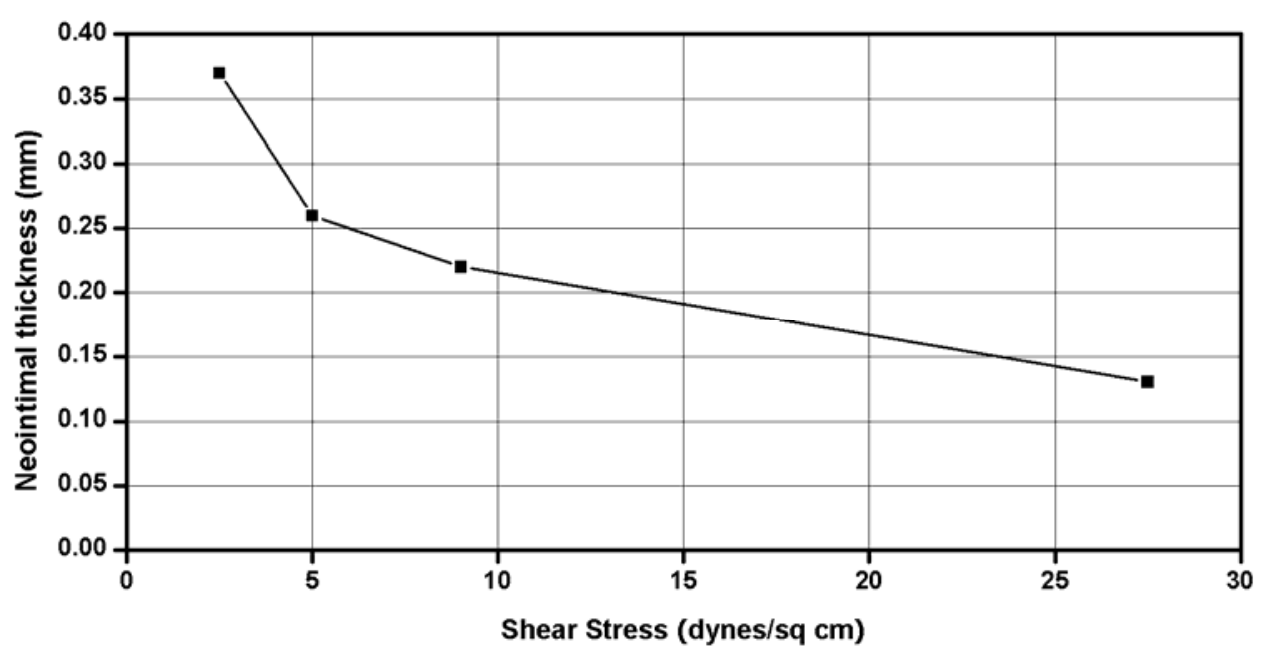

Fig. 6.5 Graph of neointimal thickness versus mean shear stress for PTFE tapered grafts in a canine model of intimal hyperplasia (from Ku and Zhu, 1993, with permission).

The OSI distributions for all the cases are displayed in Fig. 6.7. It is shown that the standard case exhibits the lowest OSI level with slightly elevated OSI at the heel and on the lateral wall of the sleeve. Reducing the proximal outflow has an acute effect on the OSI levels. Most distinguished is the zero proximal outflow case, which shows remarkably high OSI on the surface of the entire proximal outlet segment. Increasing the proximal outflow gradually increases the OSI level on the upper sleeve wall. Generally, high OSI is restricted within the sleeve for all the cases of $10 \% \sim 40 \%$ proximal outflow.

The SWSSG variations on the floor are directly compared in Fig. 6.8. The peak magnitude is slightly higher in all the nonstandard cases than in the standard one. As the proximal outflow increases, the region of elevated SWSSG is gradually extended proximally. The overall level of SWSSG is highest in the case of $40 \%$ proximal outflow. However, for all the cases investigated, high SWSSG (with normalized magnitude >1) is distributed on the sleeve surface instead of the native artery. 

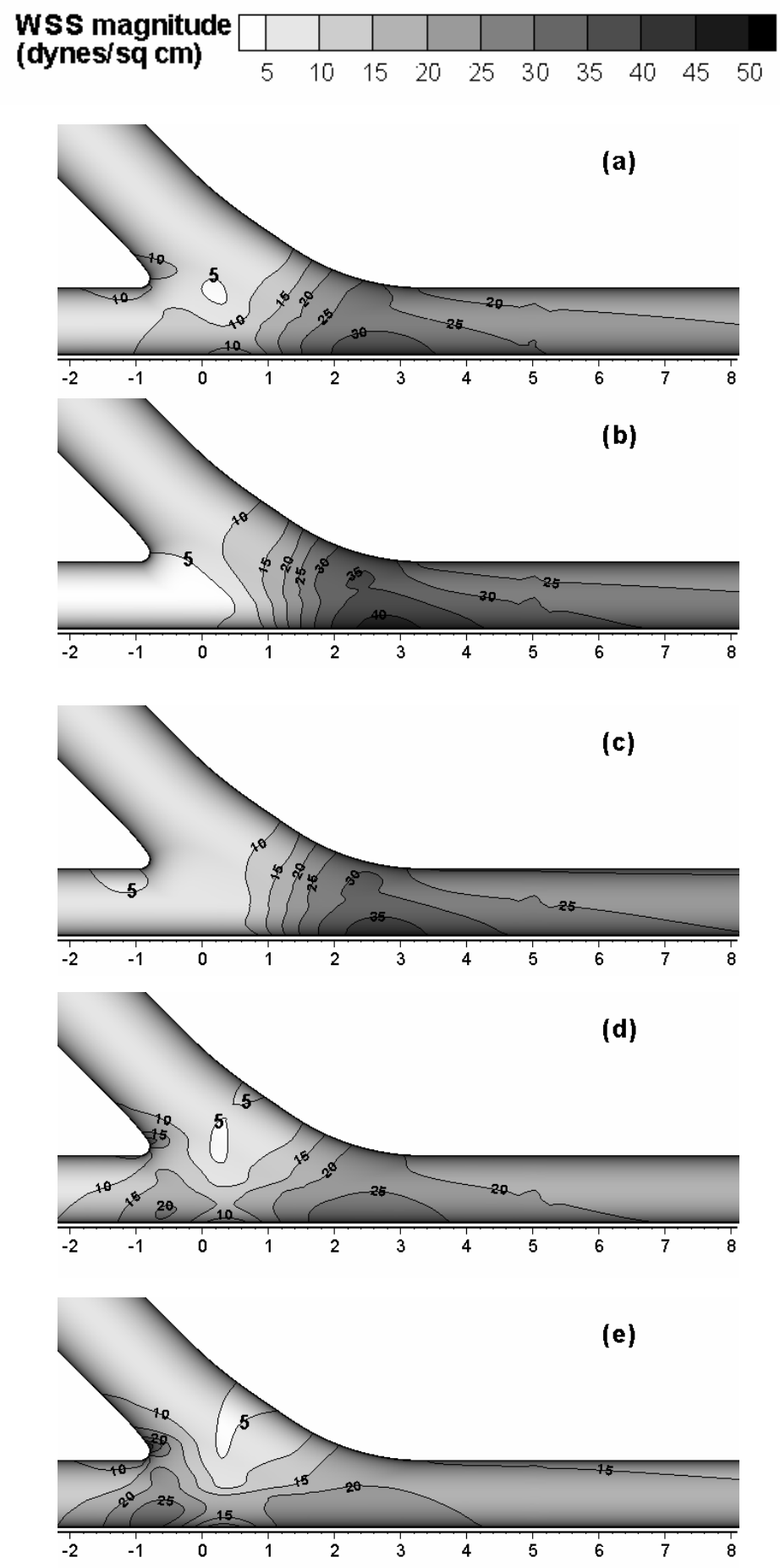

Fig. 6.6 WSS distributions on the sleeve model for (a) the standard case ( $Q_{p}=20 \%$ ), (b) $Q_{p}=0$, (c) $Q_{p}=10 \%$, (d) $Q_{p}=30 \%$, and (e) $Q_{p}=40 \%$. 

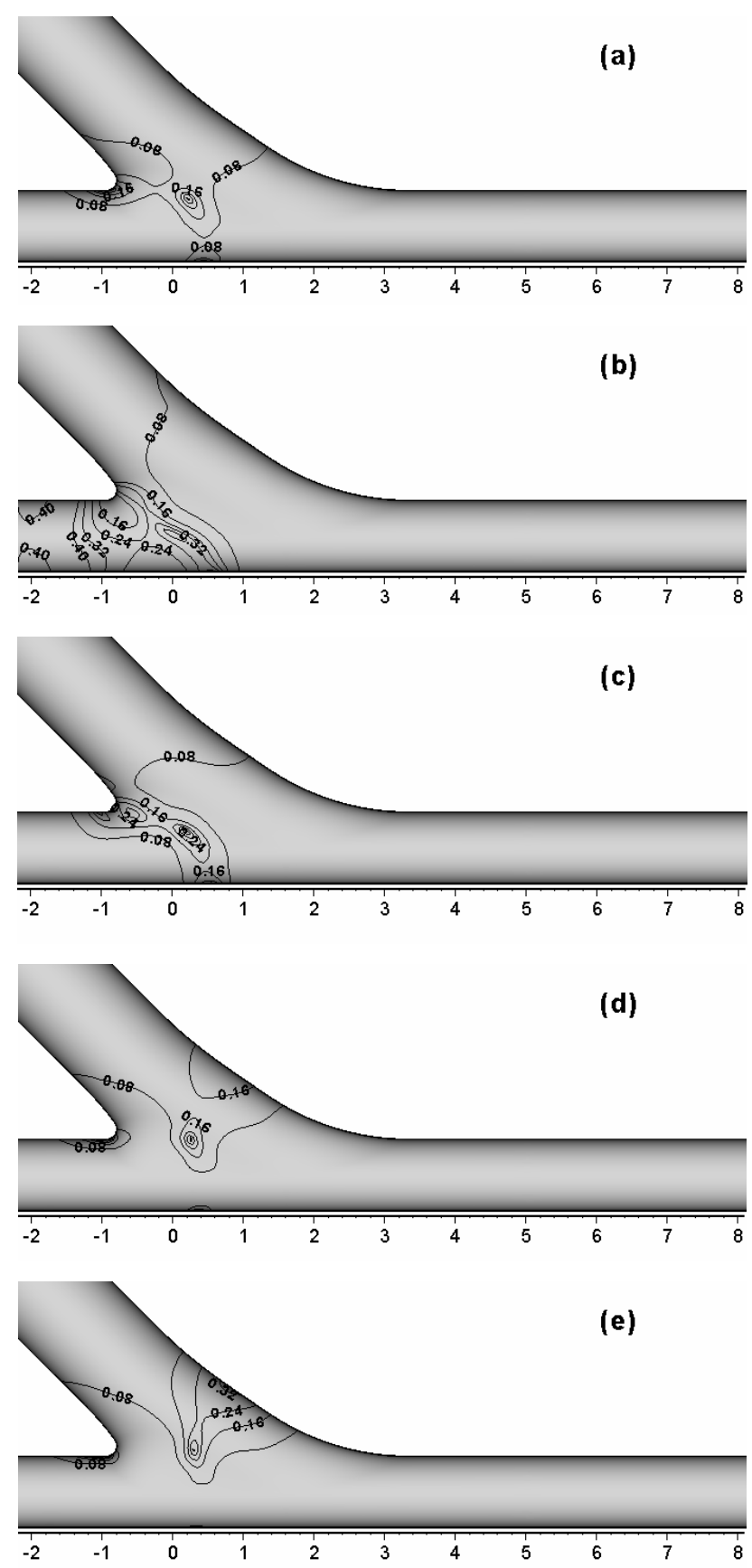

Fig. 6.7 OSI distributions on the sleeve model for (a) the standard case ( $Q_{p}=20 \%$ ), (b) $Q_{p}=0$, (c) $Q_{p}=10 \%$, (d) $Q_{p}=30 \%$, and (e) $Q_{p}=40 \%$. 


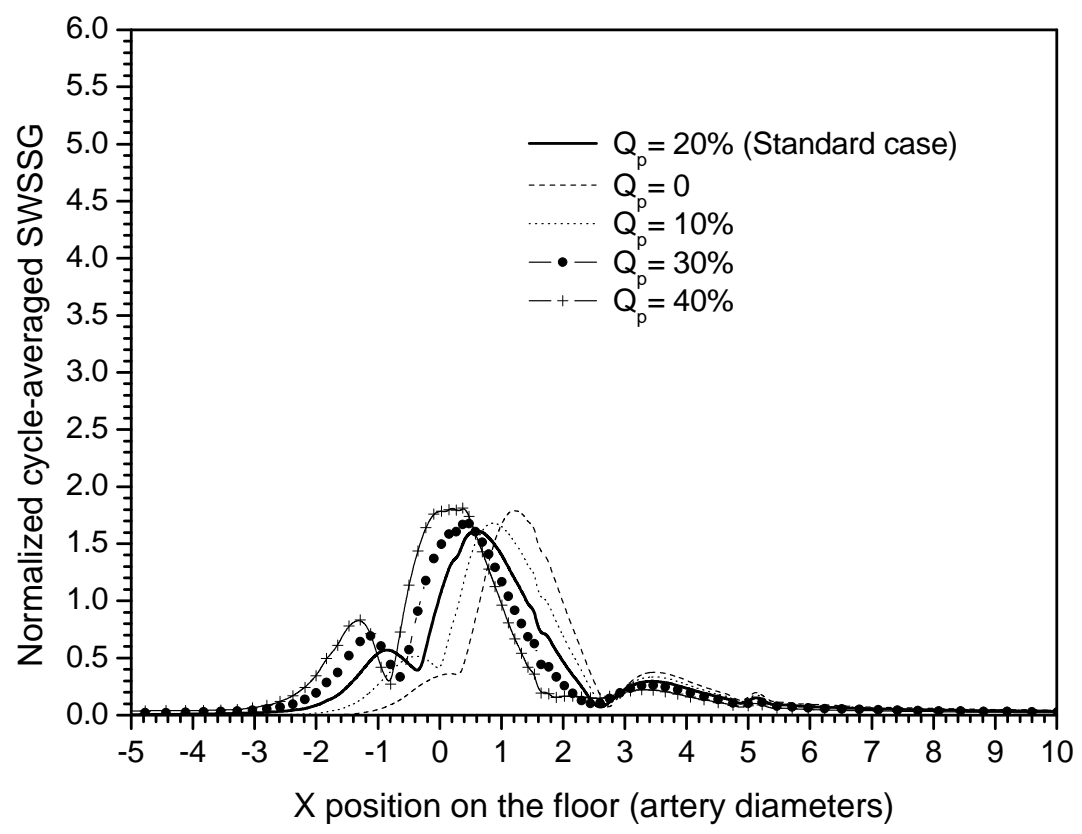

Fig. 6.8 Comparison of cycle-average SWSSG between the cases with different proximal-distal outflow ratios.

\subsection{EFFECT OF GRAFT-ARTERY NONPLANARITY}

\subsubsection{Numerical Model}

Nonplanarity is a primary feature of the anastomosis, i.e. the two centerlines of the graft and the host coronary artery lie out of plane. Nonplanarity in inflow geometry was shown to have certain influence on distributions of mean shear magnitude and oscillatory shear magnitude (Papaharilaou et al. 2002). It is therefore of interest to examine how different degrees of nonplanarity between the graft and artery affect blood flow behavior in the sleeve. Two nonplanar angles, i.e. $\beta=30^{\circ}$ and $60^{\circ}$, were investigated. 


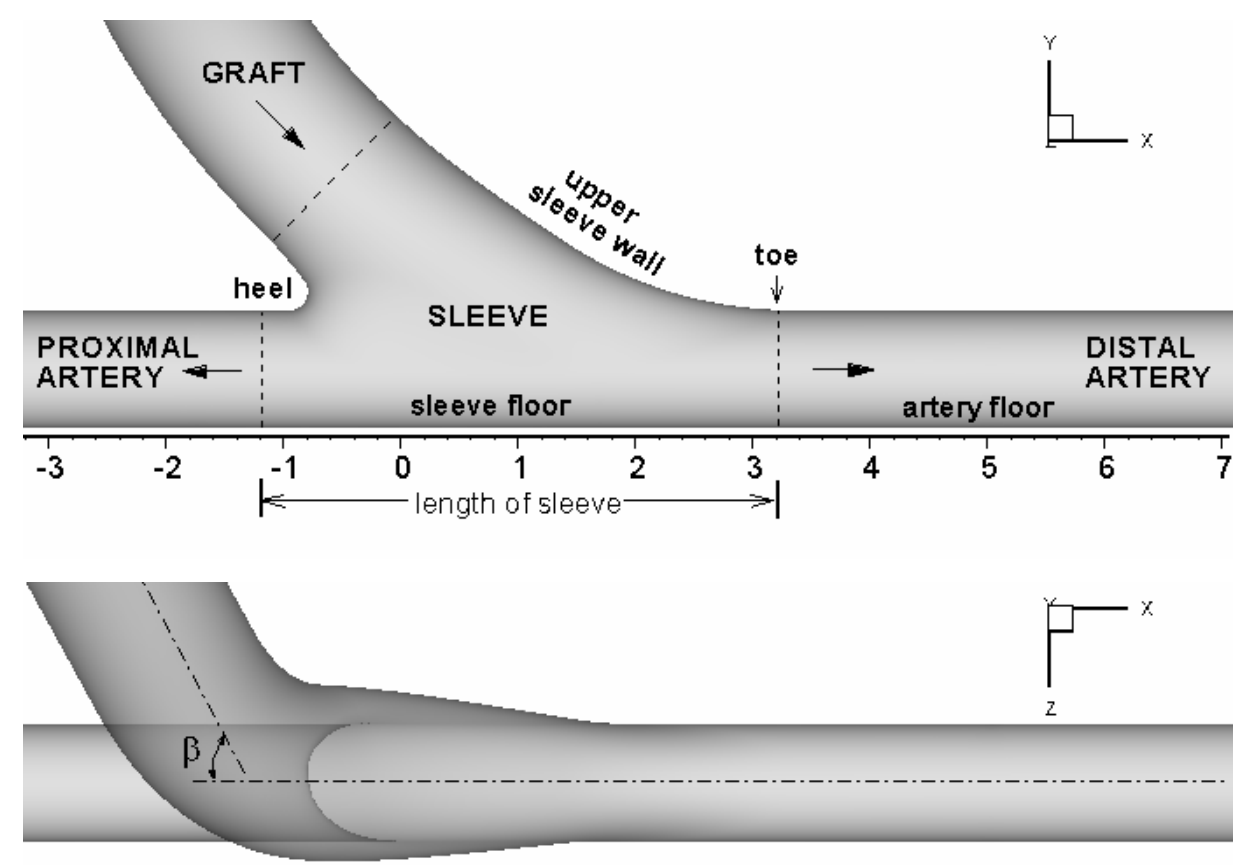

Fig. 6.9 Illustration of the sleeve model with nonplanar inflow geometry. Here $\beta$ is the nonplanar angle.

\subsubsection{Results}

The velocity profile across the blood vessel is symmetric in the planar model, whereas the nonplanar inflow geometry breaks flow symmetry and sets up a Dean-type flow. Figure 6.10 compares the distributions of axial velocity component in three typical cross-sections between the planar and $60^{\circ}$ nonplanar models. The effect of the nonplanarity is evident in the outflow vessels and decays as increasing the downstream distance. For both models, there is no sign of flow separation along the distal outflow segment. There is also distinct difference in the secondary cross-flow patterns between the two models, especially at the peak flow as shown in Fig. 6.11. In the planar model, two symmetric clockwise rotating vortices are demonstrated in the tranverse plane. In the nonplanar case, however, these two vortices are replaced by a single strong one with its core slightly deviated from the center of the cross-section. In addition, the motion of 
the stagnation point in the nonplanar model follows a three-dimensional curved path in contrast to the straight pathline along the floor in the planar case.

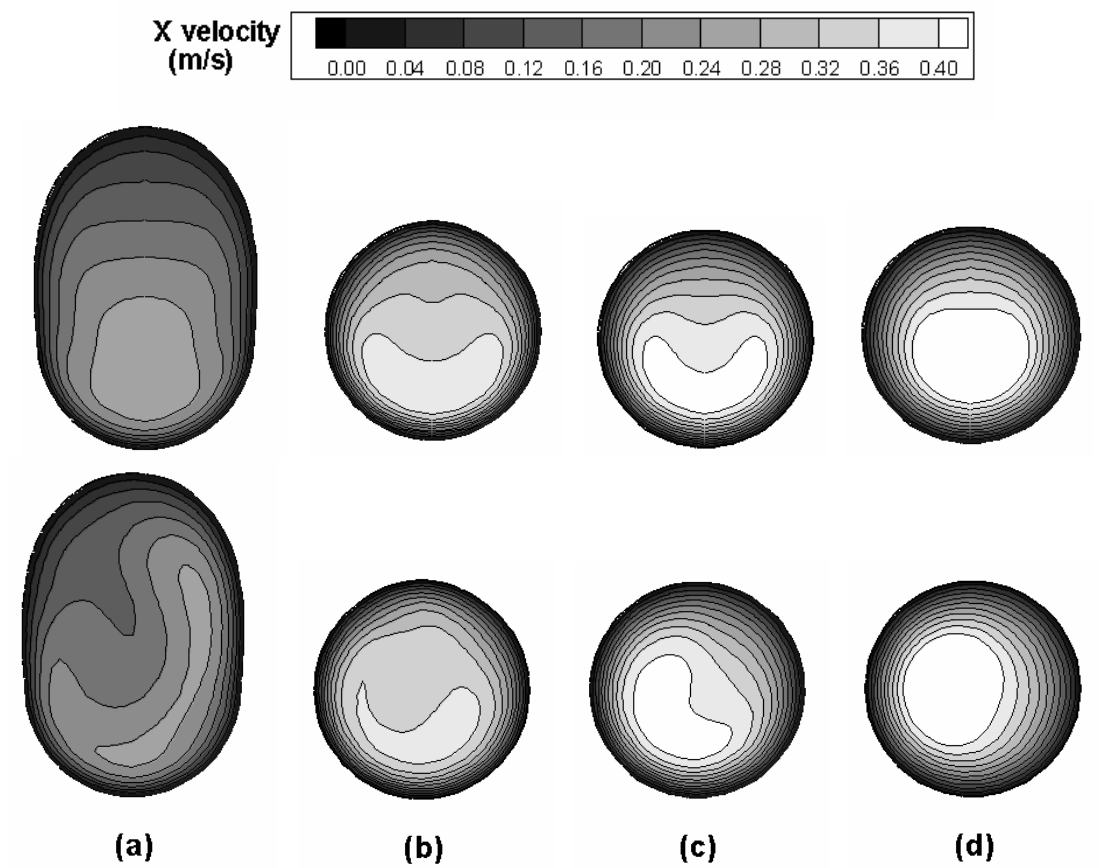

Fig.6.10 Axial velocity contour plots in the cross-sections extracted at (a) $\mathrm{X}=1.5 d_{a}$, (b) $3 d_{a}$, (c) $4.5 d_{a}$, and (d) $8 d_{a}$ for the planar (top) and $60^{\circ}$ nonplanar models (bottom) during the diastolic deceleration.

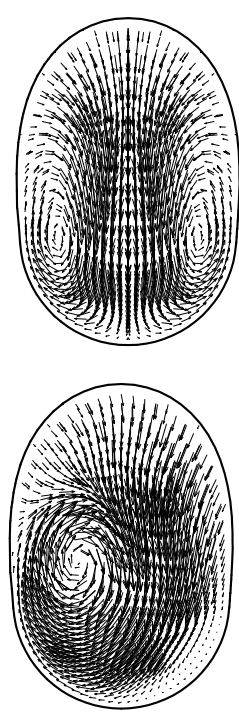

(a)
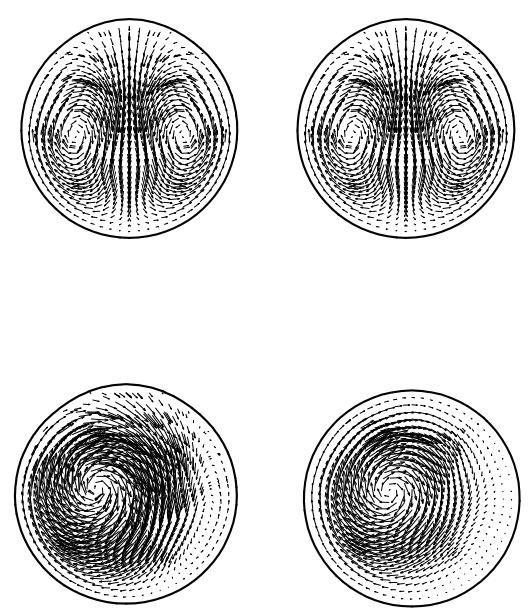

(b)

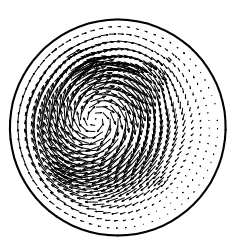

(c)
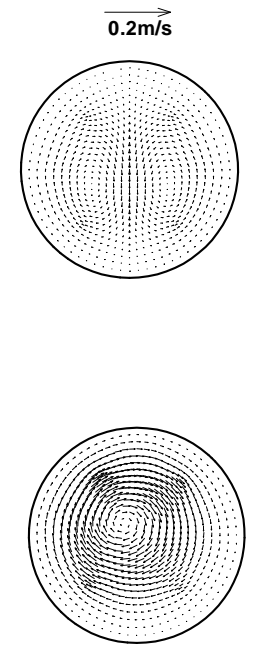

(d)

Fig. 6.11 Secondary crossflows in the cross-sections extracted at (a) $\mathrm{X}=1.5 d_{a}$, (b) 3 $d_{a}$, (c) $4.5 d_{a}$ and (d) $8 d_{a}$ for the planar (top) and $60^{\circ}$ nonplanar models (bottom) at the peak flow. 
The introduction of nonplanarity in the inflow vessel results in significant difference in the cycle-averaged WSS and OSI distribution patterns (Fig. 6.12). In the planar model, low WSS $\left(<5\right.$ dynes $\left./ \mathrm{cm}^{2}\right)$ is symmetrically distributed in small areas at the lateral wall and heel of the sleeve. Increasing the nonplanar angle tends to remove the low shear area from the outer wall of the sleeve but enlarge the area at the inner wall. The overall severity of low shear is generally higher in the nonplanar cases. Note that in the $60^{\circ}$ nonplanar model the two low shear areas at the inner wall and heel of the sleeve are most prominent.

The OSI, by definition, is correlated with low and oscillatory shear. The localizations of elevated OSI closely resemble those of low shear stress (Fig. 6.13). In the planar model, elevated OSI is associated with the heel, floor as well as the entire inflow segment. As increasing the nonplanar angle, elevated OSI shifts from the outer wall and floor of the sleeve, to the inner wall just downstream the nonplanar bend. The areas of elevated OSI in the two nonplanar models are relatively larger. For all the models investigated, the distal half of the sleeve and the entire distal artery are devoid of elevated OSI. 

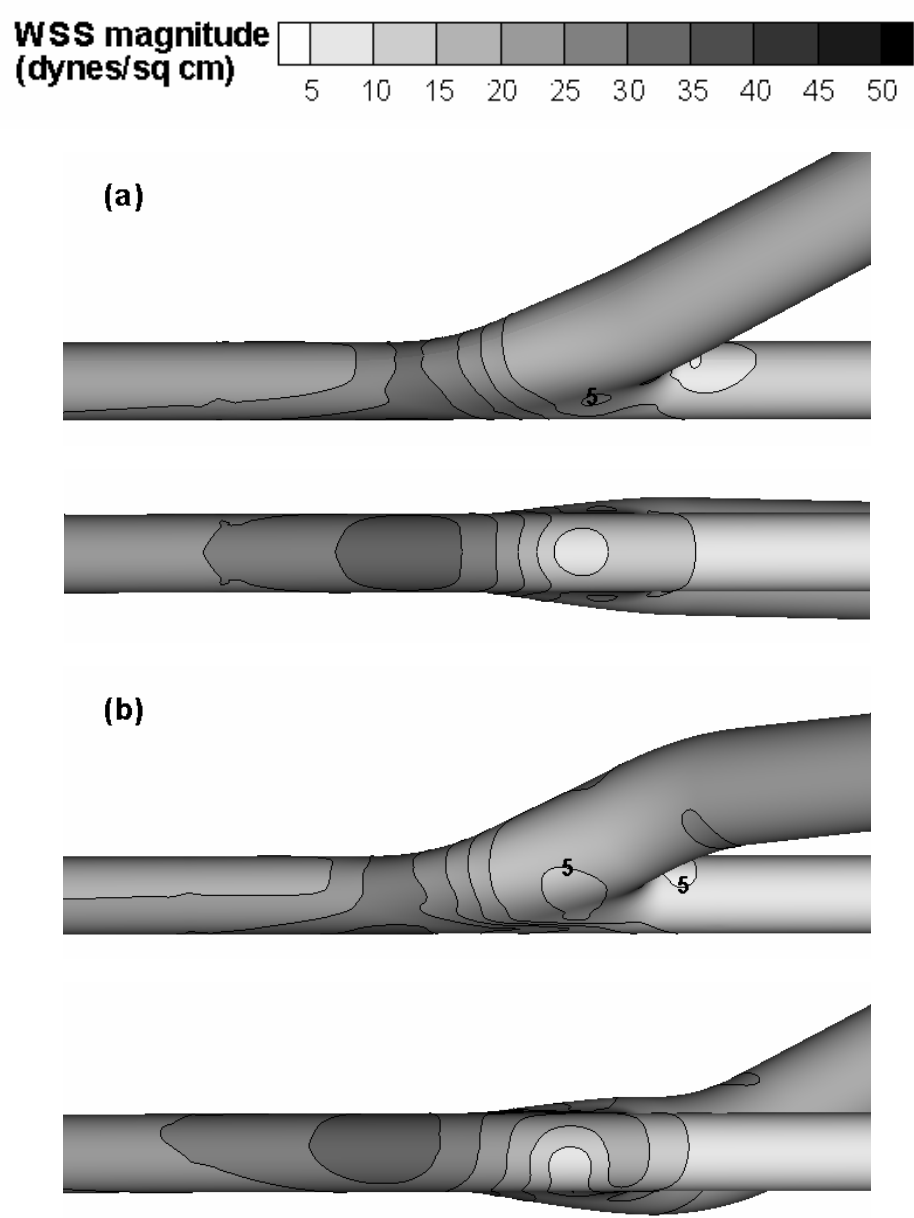

(c)

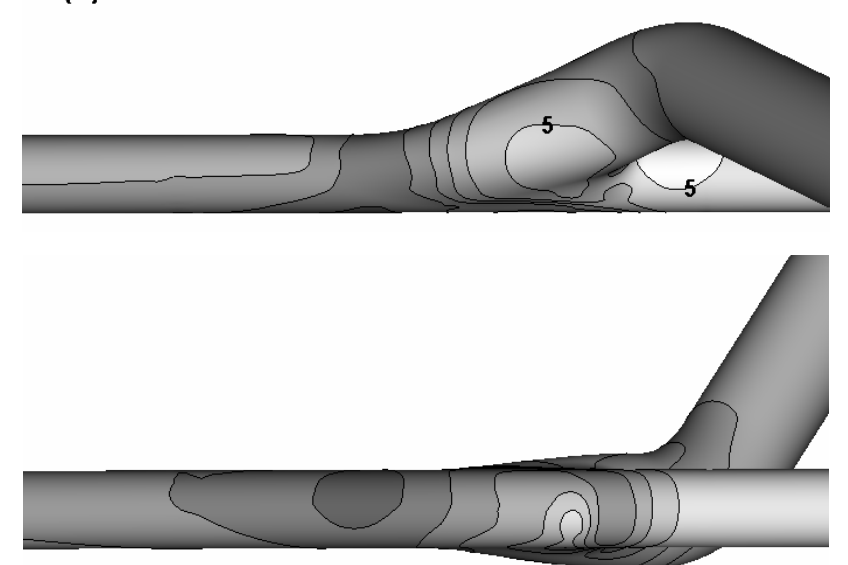

Fig. 6.12 WSS distributions on the model surface for the models with (a) $\beta=0$, (b) $\beta$ $=30^{\circ}$, and (c) $\beta=60^{\circ}$. For each model, two different views are displayed. 

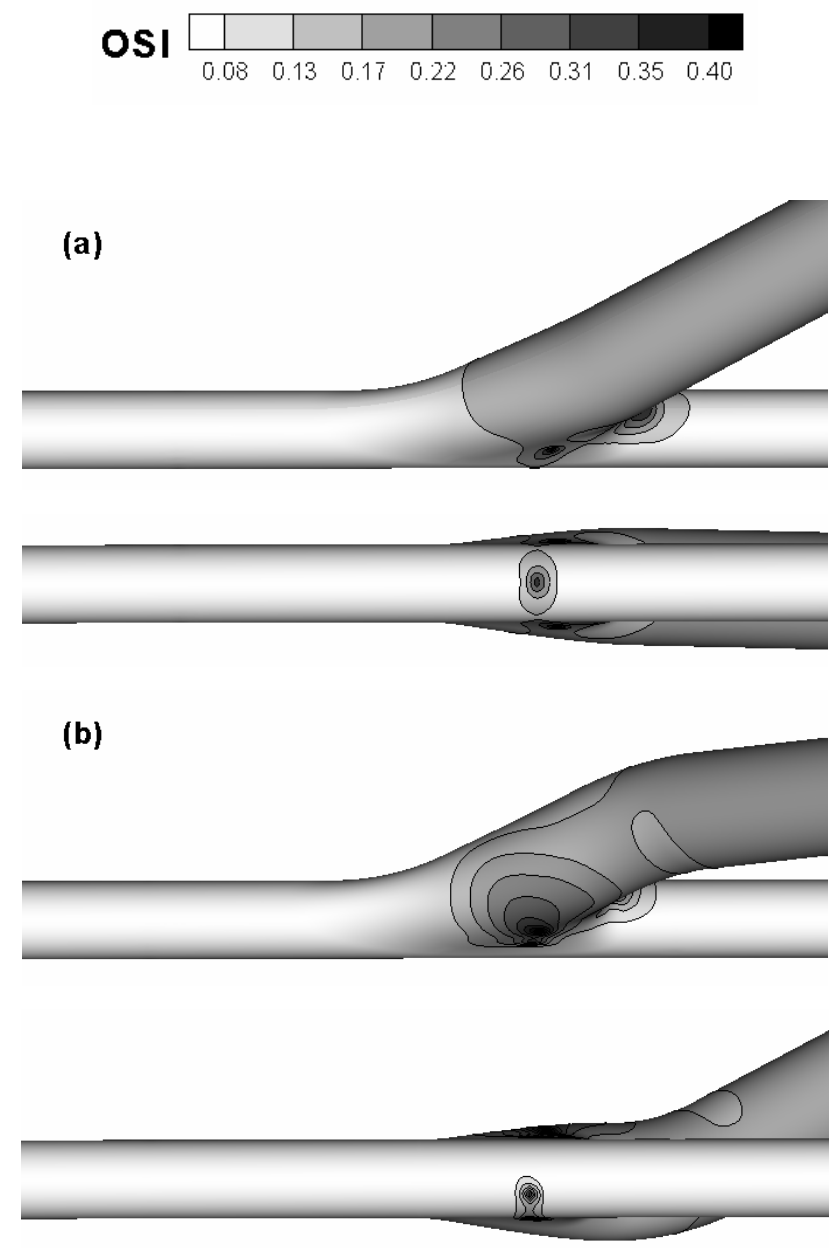

\section{(c)}

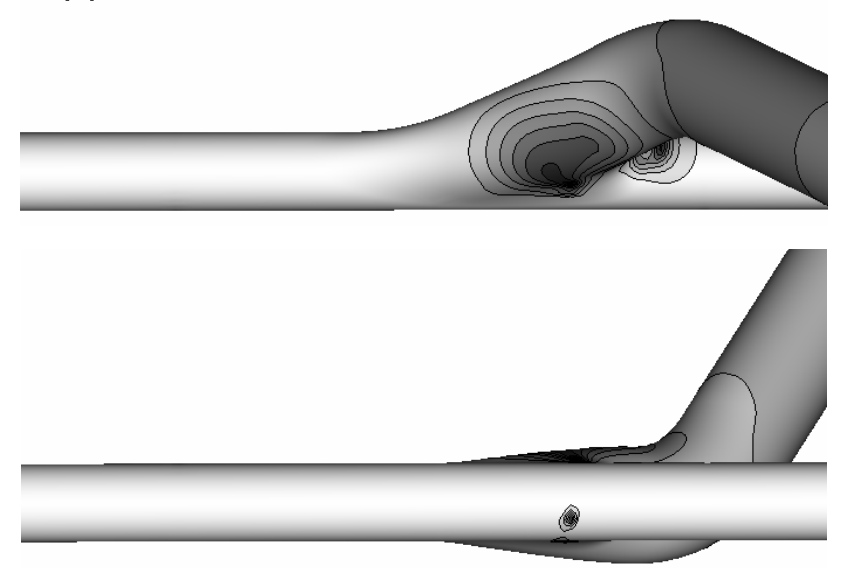

Fig. 6.13 OSI distributions on the model surface for the models with (a) $\beta=0$, (b) $\beta=$ $30^{\circ}$, and (c) $\beta=60^{\circ}$. For each model, two different views are displayed. 


\subsection{EFFECT OF SLEEVE WALL THICKNESS}

\subsubsection{Numerical Model}

On inserting the sleeve into the graft and recipient artery, a step will be created at each end of the sleeve as shown in Fig. 6.14 and may exert an adverse effect on the local flow behavior. This effect should not be significant if the step size, which is equal to the thickness of the sleeve wall, is sufficiently small. That is, there must be a threshold for the step size, beyond which the sleeve performance would be effetively compromised. Distributions of wall parameters are examined when gradually increasing the step size from $0.05 d_{a}$ to $0.01 d_{a}$.

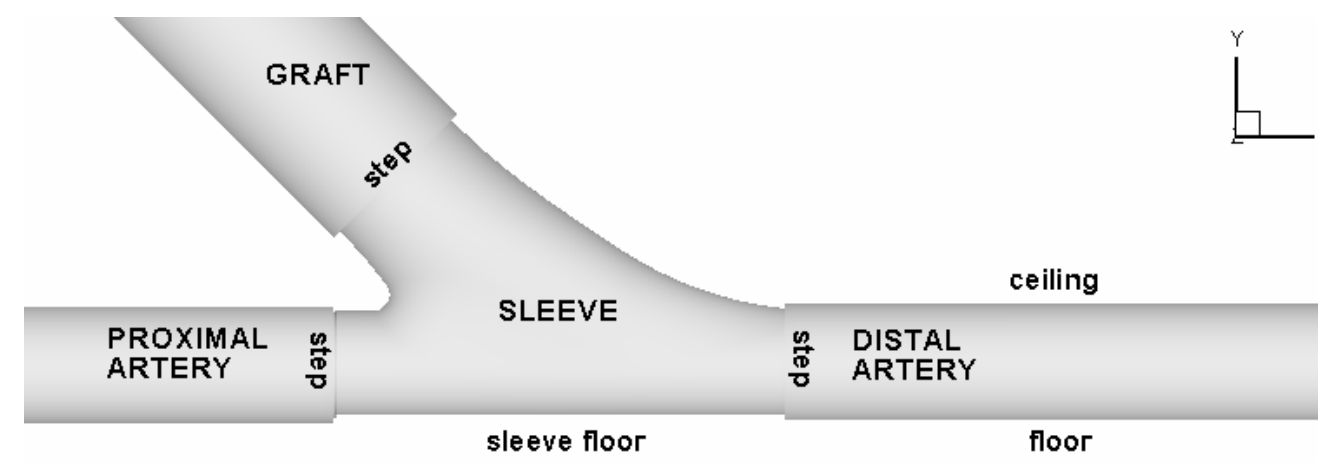

Fig. 6.14 Illustration of the sleeve model with steps on the ends.

\subsubsection{Results}

Increasing the thickness of the sleeve wall induces no appreciable changes to the WSS and OSI distributions on the sleeve surface, whereas the changes around the steps (especially at the sleeve-distal artery step) are prominent. These phenomena can be observed in Fig. 6.15, which shows OSI contour plots for all the three models. As compared to the idealized model without steps, the OSI level around the distal step is only slightly increased in the models with a thickness of $0.05 d_{a}$ and $0.08 d_{a}$. Further increasing the thickness to $0.1 d_{a}$, however, causes an abrupt increase in both the magnitude and area of the elevated OSI at the entrance of the distal artery segment. 

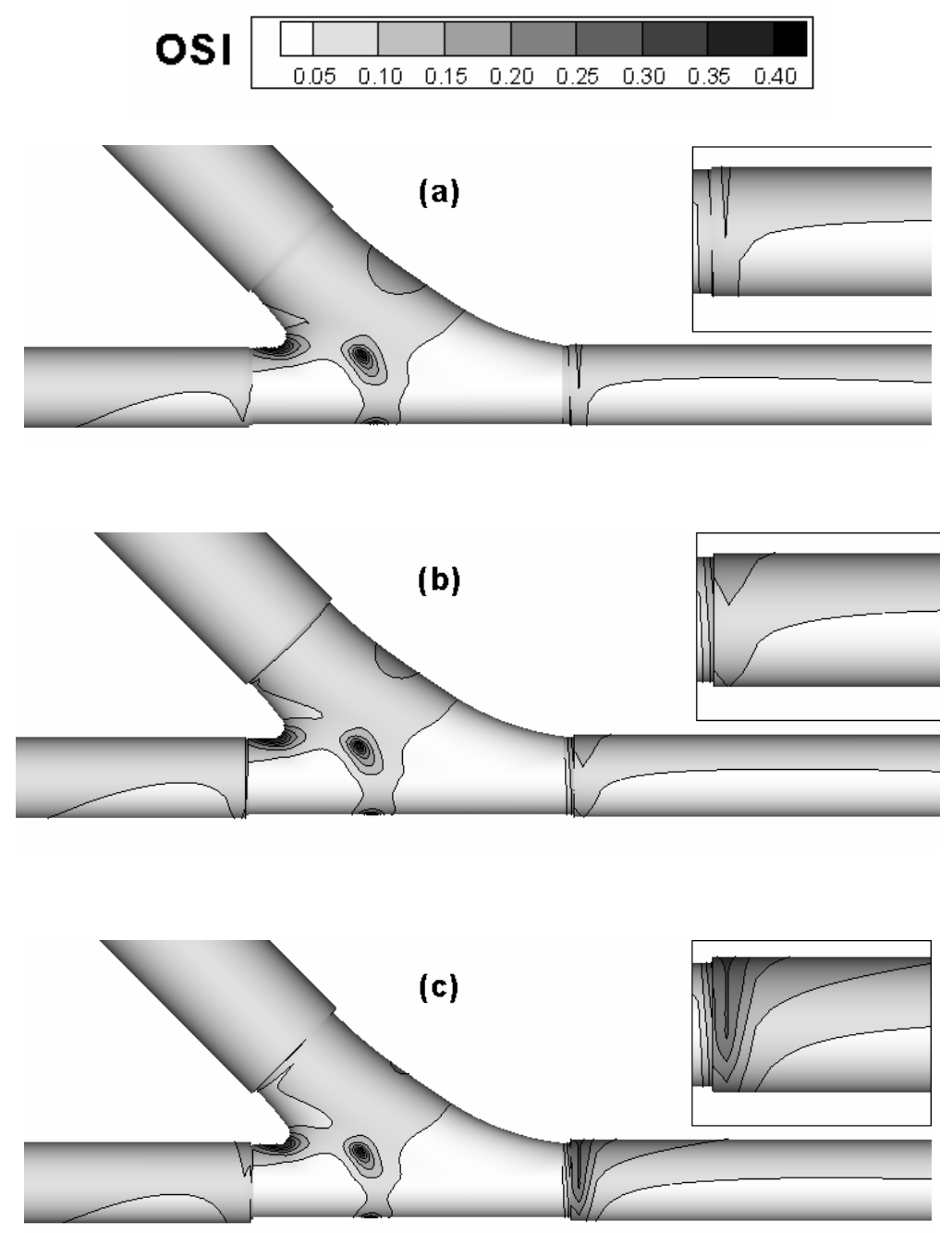

Fig. 6.15 OSI distributions on the model surface for the models with step sizes of (a) $0.05 d_{a}$, (b) $0.08 d_{a}$, and (c) $0.1 d_{a}$. The small panel shows the close-up view at the sleeve-distal artery step. 


\subsection{DISCUSSION}

The most important contribution of the sleeve technique relies in the fact that the sleeve device can reproduce and retain the hemodynamically conducive geometry in vivo, which is essential for the long-term patency of bypass grafts. To ensure that its geometry accurately follows the designed data, the sleeve must be prefabricated with synthetic material and provided off-the-shelf, which would inevitably introduce foreign material to the human body. Therefore, it is important to be aware that the employment of the sleeve device should not create more problems than it solves. The inherent disadvantage of synthetic material is its vulnerability to acute thrombosis causing rapid occlusion in the vessel lumen. Seeding with endothelial cells and subsequent proliferation of these cells into a confluent monolayer along the synthetic surface is suggested as an attractive solution to reduce thrombogenicity in recent years. Several complementary approaches to enhance the results of endothelial cell seeding have also been developed, including in vitro and in vivo conditioning of the cells and modification of the cell phenotype through gene transfer. The purpose is to induce a suitable functional phenotype in the cells and to maintain it despite adverse signals on synthetic surfaces (e.g. from inflammatory stimuli or mechanical injury) (Canfield and Schor, 1995). However, the results of cell seeding have been variable and the clinical utility remains to be defined. Another solution to inhibit thrombus formation is to regulate local hemodynamics, which is found to be closely associated with platelet activity and thus may play critical roles in thrombogenesis. It was shown that platelet aggregation on collagen-free artificial surfaces is promoted by recirculating flow and low shear magnitude (Schoephoerster et al., 1993). In this point, minimizing areas of flow recirculation and low shear on synthetic surfaces is expected to reduce the likelihood of thrombus formation. 
In addition to thrombus formation, synthetic material may develop neointimal hyperplasia leading to a progressive intimal thickening near the interfaces to the native vessels. The sequence of neointimal hyperplasia is characterized by platelet and thrombin/fibrin adhesion on the artificial surface, smooth muscle cell migration from the native artery into the synthetic vessel, and synthesis and deposition of large amounts of extracellular substances (Glagov et al., 1991). Hemodynamic shear stress is postulated to be responsible for the abnormal tissue ingrowth on the synthetic surface. An inverse, nonlinear relationship between neointimal thickening and WSS is demonstrated by studying in vivo PTFE grafts. It is therefore suggested that increasing WSS and minimizing area of sustained low shear on the synthetic surface not only reduces the tendency towards rapid occlusion due to thrombosis, but it is also expected to delay late occlusion as a result of neointimal hyperplasia. The area and severity of low WSS on the sleeve surface would be one of the important criteria for the assessment of sleeve performance.

The sleeve is supposed to cover all the disease susceptible sites and at the same time its surface area should be kept as small as possible. That is to say, the sleeve should act as a flow streamliner and a protector to the native artery with the minimum length. This would be another criterion for assessing a sleeve device. In the present study, the distributions of the primary indicators such as WSS, OSI and SWSSG were compared for the native vessels. It was discussed previously that low WSS and high OSI are associated with increased aggressiveness in endothelial cell morphology, greater intimal and smooth muscle cell proliferation, increased surface concentration of LDL, etc. High SWSSG, on the other hand, may cause endothelial regression and enhance permeability 
of the endothelium to macromolecules. Thus, low WSS, high OSI and high SWSSG should be completely eliminated in the native artery.

The present study examined the sensitivity of the sleeve to altered proximal flow conditions. The nature of the flow in the segment of artery proximal to the distal anastomosis varies depending on the severity of the stenosis and the location of the anastomosis. Although less surgically convenient, placing the graft immediately after the stenosis was suggested to be favorable for better preservation of the proximal artery and its branches (Guo et al., 2001). If the stenosis is completely occlusive, there would be zero outflow to the proximal artery. In many situations, however, surgeons routinely anastomose the graft to the distal portion far downstream the stenosis since the proximal portion of the vessel is often buried in the epicardial fat and even in the myocardium. As a result, a long segment of native artery and its tributaries are located between the blockage and the distal anastomosis, which receive blood from the graft in a retrograde fashion (Guo et al., 2001). The portion of the proximal outflow is different among individuals and is also likely to vary over the cardiac cycle. Thus, for an ideal anastomotic geometry, its hemodynamic performance should not easily be compromised by normal changes in physiological flow conditions. The comparison between different outflow ratios in this study shows that the total area of low WSS $(<5$ dynes $/ \mathrm{cm}^{2}$ ) is not appreciably enlarged in the cases of $10 \% \sim 40 \%$ proximal flow as compared to the standard case. For zero proximal flow, however, the proximal half of the sleeve surface is characterized by persistent flow recirculation and low shear, which suggests a higher tendency towards thrombus formation as well as neointimal thickening. In addition, for all the cases investigated high SWSSG is restricted within the sleeve area. These findings suggest that the sleeve with the designed geometry is not sensitive to the outflow ratio changes, and suboptimal performance is only associated 
with the case of zero proximal flow. Although the flow recirculation and low shear is located in the less critical proximal region for the zero proximal flow case, its effect should not be neglected. For example, a thrombus formed on the artificial sleeve surface may be detached and migrate to occlude other parts of the vascular system leading to stroke and transient ischemic attack (Constantinides, 1990). Also, once neointimal thickening begins to grow, the effect may snowball. In this regard, for a completely occlusive stenosis in the proximal region, the sleeve should involve only the distal outlet similar to an end-to-end anastomosis; that is, the proximal outlet should be replaced by a streamlined wall.

Nonplanarity is a common feature in natural and reconstructed bends and bifurcations. The purpose of the present study is therefore to examine if the sleeve performance would be compromised by nonplanar inflow geometry of the graft. The numerical results show that increasing the nonplanar angle causes the low shear area on the sleeve surface to be enlarged and shift upstream. This effect is especially significant in the case of $\beta=60^{\circ}$. It is also important to note that the nonplanar inflow shifts the high OSI area from the sleeve floor and lateral wall upstream of the graft-sleeve interface, and the $60^{\circ}$ case gives the highest overall OSI level. Therefore, caution is advised in applying the sleeve device to the nonplanar anastomosis. The anastomosis should be designed in such a way that the nonplanar bend of the graft is located at a sufficient distance from the inlet of the sleeve. This study also suggests the importance of accurately reproducing the nonplanar curvature that is located near the anastomosis for the purpose of disease prediction. In this point, assuming symmetric inflow geometry would lead to inaccurate prediction of disease-susceptible sites and would underestimate the severity of disease. It should also be understood that the geometry and location of nonplanar curvature are patient-specific features and cannot be 
generalized. For studies that try to correlate hemodynamics with disease pathology, if detailed information of patient-specific geometric features and relevant pathological data of disease distribution are known, then it is necessary to reproduce the nonplanar curvature and helical inflow to allow precise correlations. Without this information, however, it seems acceptable and more convenient to assume symmetric inflow to provide a general description of the anastomotic flow field.

Choosing an appropriate wall thickness is critical in designing the sleeve device. It is shown that local hemodynamics at the step of the sleeve-distal artery interface is sensitive to the sleeve wall thickness. Increasing the thickness from $0.08 d_{a}$ to $0.1 d_{a}$ causes an acute effect on the OSI level at the entrance of the distal artery. This finding suggests that the thickness of the sleeve wall should not exceed $0.08 d_{a}$ so as not to result in high OSI in the distal native vessel.

The sleeve technique proposed here seems attractive for its multiple advantages to solve problems in coronary vascular reconstructions. The sleeve device is expected to be particularly useful for patients where autogenous veins and arteries are not available. In this case, the graft and the sleeve can be integrated during fabrication. The present study has only focused on hemodynamic aspects of the sleeve device. To make the full potential of the technique, several technologies need to be developed or further improved. First, there is a need for a new synthetic material or cell seeding approach to reduce the likelihood of acute thrombus formation. This material should offer ease of fabrication and have similar elastic properties to the native artery. Second, it is advisable to use biologic glue as complementary or full support to affix the sleeve to the inner surfaces of the graft and artery, by taking advantage of the overlapping vessel margins. The use of glue would not only expedite the surgical procedure but it would 
also relieve stress concentration around the suture lines. However, biologic glues currently available are not strong enough to provide full structural support. Most important, a sleeve delivery system needs to be developed to manage precise placement of the sleeve device and thus standardize the surgical procedure. A standardized procedure would then allow totally endoscopic or robotic execution. The author is also aware of the disadvantages of the sleeve technique such as introducing foreign materials and covering part of the native vessel. In infrainguinal arterial reconstructions, an ePTFE cuff prosthesis preshaped with desired geometry has proved to have superior success rate to any of its contemporary techniques (Oderich et al., 2005; Panneton et al., 2004). The promising results suggest that the advantages of improved anastomotic geometry and simplified implantation procedure of the synthetic prosthesis could outweigh the drawbacks of the foreign material introduced. It is therefore expected that a sleeve prosthesis would similarly benefit the patients undergoing CABG, particularly for those who have no suitable autologous veins or arteries. Only the long-term patency will reveal the future of this technique. 


\section{CHAPTER 7}

\section{EXPERIMENTAL VALIDATION OF NUMERICAL RESULTS}

\subsection{INTRODUCTION}

Anastomotic hemodynamics has been studied in the last several decades using a variety of approaches including in vivo and in vitro experimentations, numerical simulation, and analytical solution. Numerical simulation is an attractive tool for investigating the relationship between hemodynamic parameters, anastomotic geometry (particularly for distal end-to-side anastomoses), and the development of vascular diseases (Fei et al., 1994; Moore et al., 1999; Lei et al., 1997). However, in applying numerical simulation it is essential to properly validate and test the in-house code against experimental methods to verify the accuracy of the numerically predicted results. Different metrological tools for in vitro experimental studies of flow fields have been employed and have experienced much improvement. The earlier information in this field has been accumulated primarily by qualitative flow visualization or dye studies (White et al., 1993; Ethier et al., 1998a). Much recent efforts have been dedicated to the development of noninvasive techniques using optical probes for quantitative velocity measurements. The most promising techniques are the laser-Doppler anemometry (LDA) and particle imaging velocimetry (PIV). Both rely on the presence of small tracer particles in the flow and assume that the particles' motion faithfully resembles the fluid motion. In contrast to the LDA technique for single-point velocity measurement, the PIV allows the spatial velocity distribution over the entire flow domain to be visualized as well as quantified and thus has been gaining a growing popularity. For example, Bates et al. 
(2001) investigated two-dimensional instantaneous velocity fields inside a $30^{\circ} \mathrm{Y}$ junction under different steady flow conditions using water as the working fluid, and clearly identified complex flow features and the formation of vortices. Heise et al. (2003) via high-speed digital PIV measured complex pulsatile flow fields in a silastic model of distal end-to-side anastomosis. Based on the well-resolved velocity data, additional flow dynamical features such as wall shear rate, wall shear stress and vorticity were also calculated. The results have confirmed that the locations of low shear areas correspond to the well-known regions of intimal hyperplasia. Subsequently, Heise et al. (2004) examined the effect of three different configurations of distal femoro-crural anastomoses representing a Taylor patch, a Miller cuff and a femorocrural patch prosthesis by means of PIV measurements. Distinct differences in detailed flow patterns between these models were detected, such as a significant clockwise rotating vortex inside the ceiling separation zone of the Taylor patch anastomosis and a large anti-clockwise rotating vortex inside the large heel separation of the Miller cuff anastomosis. All these studies have demonstrated that the PIV is a competent and reliable tool for quantitative velocity records of good accuracy.

As far as the author is aware, this is the first study in employing the PIV technique to study pulsatile flows in life-size coronary anastomosis. Instantaneous velocity vector fields in two different end-to-side anastomotic models were measured. The results were compared with the numerical data in both velocity fields and derived WSS variations based on the same model geometries. 


\subsection{EXPERIMENTAL METHOD}

\subsubsection{Experimental Model}

Two 1:1 clear silicone rubber models were fabricated for the present experimental study, representing the anastomoses in the LAD and RCA regions, respectively (Fig. 7.1). For the validation purpose, the model geometries strictly conform to those for the simulations. The artery diameter is $3.3 \mathrm{~mm}$ for the LAD and $2 \mathrm{~mm}$ for the RCA. For both models, the diameter of the graft is about 1.5 times the diameter of the recipient artery. The model for the LAD has smooth graft-artery transitions with an aspect ratio of 8 at the toe, corresponding to the optimized geometry; whilst the one for the RCA is based on the conventional geometry with stiff graft-artery transitions.

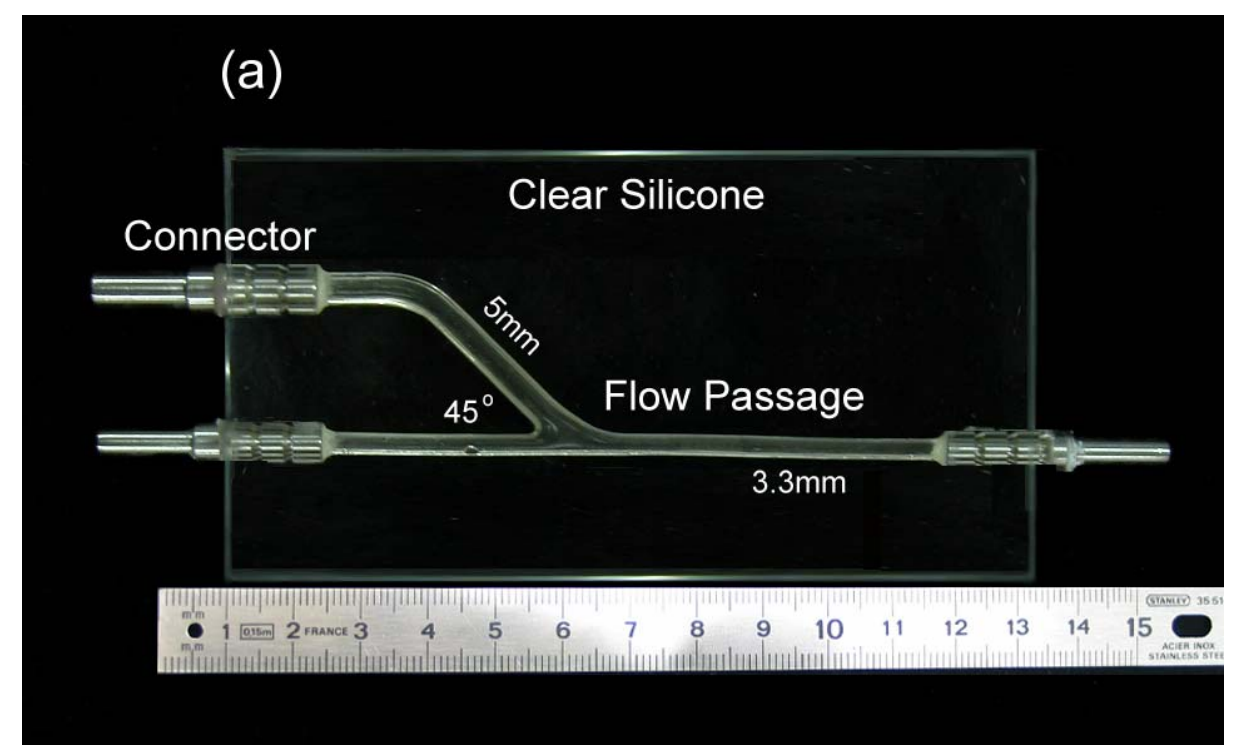




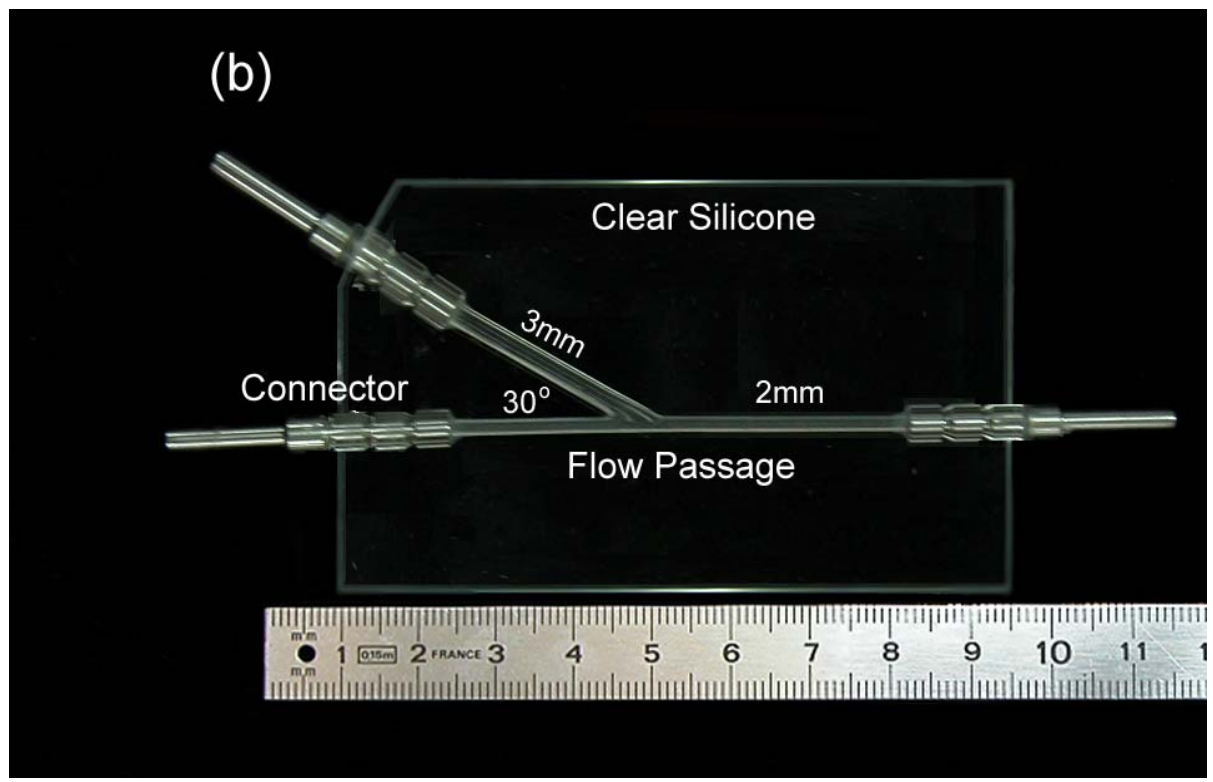

Fig. 7.1 Clear silicone rubber models representing anastomoses in the (a) LAD and (b) $\mathrm{RCA}$ regions.

The first step of building the experimental model was to create a solid computer model that describes the 1:1 replica of the flow passage using the computer-aided design software Pro/ENGINEER (Parametric Technology Corporation, Needham, MA). The solid computer model was then processed in a computer numerical control (CNC) milling machine to produce an acrylic or aluminum mould. An alternative approach of this step is the employment of a rapid prototyping technique, which requires translating the computer model data to a stereolithpgraphy (STL) file to create a resin mater by means of a rapid prototyping machine, encasing the resin master in silicone elastomer, and removing the resin master to produce a silicone elastomer mould.

In the next step, the mould was used to create a solid cast by injecting a low-meltingpoint alloy. This alloy is easy to mold and surface defects can be easily repaired. The cast surface was smoothed by gentle polishing, spray painted and dried naturally. Care was taken to apply only a thin and even layer of paint so as not to significantly affect the size of the flow passage. Three ends of the cast representing the flow inlet and outlet 
boundaries were connected with stainless steel connectors, which were used for the attachment of silicone rubber tubings. The cast with connectors was then suspended in a rectangular acrylic container. The container was constructed using a combination of acetone and silicone aquarium sealant (Dow Corning) to join individual acrylic components together. First, acetone (which is perfect for agglutinating the acrylic material) was applied on every pair of contact acrylic faces, and then a coherent layer of the silicone sealant was applied along the joints to reinforce the connection and ensure impermeability. The container has flat sides to minimize optical distortion during the PIV recording stage. The distance from the cast to the edge of the container should be thin enough that the scattered light is not overly attenuated, but not so thin that the pressure in the flow passage during the experiment will damage the model. The distance was kept about $10 \sim 15 \mathrm{~mm}$ in the present study.

The silicone (Sylgard 184, Dow Corning) and curing agent were fully mixed and exposed to a vacuum oven to remove air bubbles and dissolved air. After thorough degasification, the mixture was carefully poured into the acrylic container along the wall (or through a funnel) to minimize air entrainment and allowed to cure at $60 \pm 5^{\circ} \mathrm{C}$ for 12 hours. Thereafter, the oven temperature was increased to about $90^{\circ} \mathrm{C}$ to melt the alloy. The adherent paint was cleared by gentle brushing using acetone or detergent solution. After the alloy and paint were completely dissolved, the acrylic container was stripped from the model, leaving a replica of flow passage imbedded in the clear silicone. The entire process of creating the physical model is illustrated in Fig. 7.2 (Chong et al., 1999). 


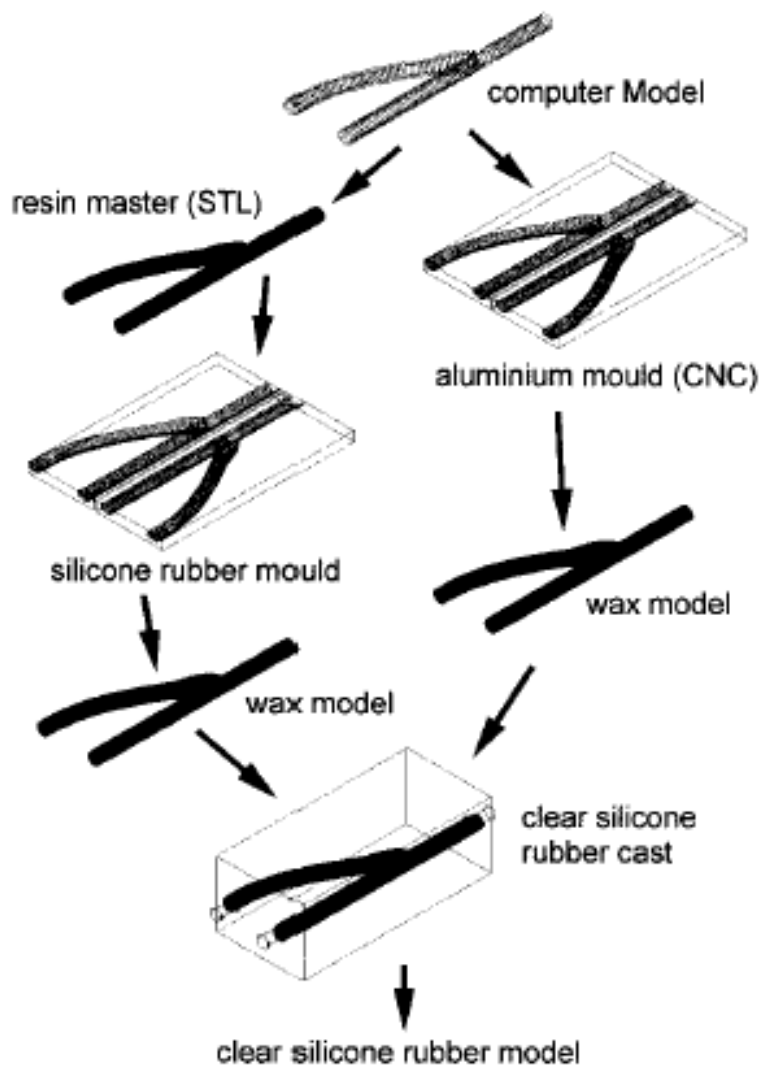

Fig. 7.2 Procedure of the physical model production (from Chong et al., 1999, with permission).

\subsubsection{Flow Circuit}

A purpose-built mock circulatory system was established. The system picture and the diagram of the flow circuit are shown in Fig. 7.3. A computer-controlled gear pump system (Micropump, Inc., Berkshire, UK) was used to generate the physiological inlet flow waveforms. The system consisted of an AC pump which generates the oscillatory component of the waveform with completely forward flow, a DC pump which operates at a constant speed to generate a steady component, and a calming chamber which serves to dampen pump-generated fluctuations. To produce bi-directional pulsatile flow, the output from the DC pump was subtracted from that from the AC pump. The pump system operated at 75 beats/min $(1.25 \mathrm{HZ})$ with the mean Reynolds number of 230 based on the host artery diameter, corresponding to mean inlet velocities of around 118 
$\mathrm{ml} / \mathrm{min}$ and $72 \mathrm{ml} / \mathrm{min}$ in the LAD and RCA models, respectively. The flow withdrawn from the reservoir by the pump system then passed through a compliance chamber, which was used to adjust the pulsatility of the waveform by changing the air-fluid volume ratio. The flow rate immediately upstream of the test section was measured and monitored by means of an electromagnetic flowmeter (Promag 53, Endress+Hauser, Germany). The signal from the flowmeter was fed back to the computer so that the pulsatility index of the waveform can be calculated and verified. The pulsatility index quantifies the shape of the waveform and is defined as the peak-to-peak amplitude of a waveform, divided by the mean amplitude over the cardiac cycle (Gosling and King, 1974). The actual mean flow rate was determined by measuring the volume of the fluid collected over a known time interval (several minutes). The experimental inlet flow waveforms conform nicely to the ones used in the numerical study (Fig. 7.4). The flow division ratio in the proximal and distal outlet segments of the test section was regulated by two needle valves (AS1000, SMC CORPORATION, Tokyo, Japan) placed downstream of the two flow outlets, respectively. The ratio was specified to be $Q_{g}: Q_{p}: Q_{d}=100: 20: 80$ based on the mean flow rate. 

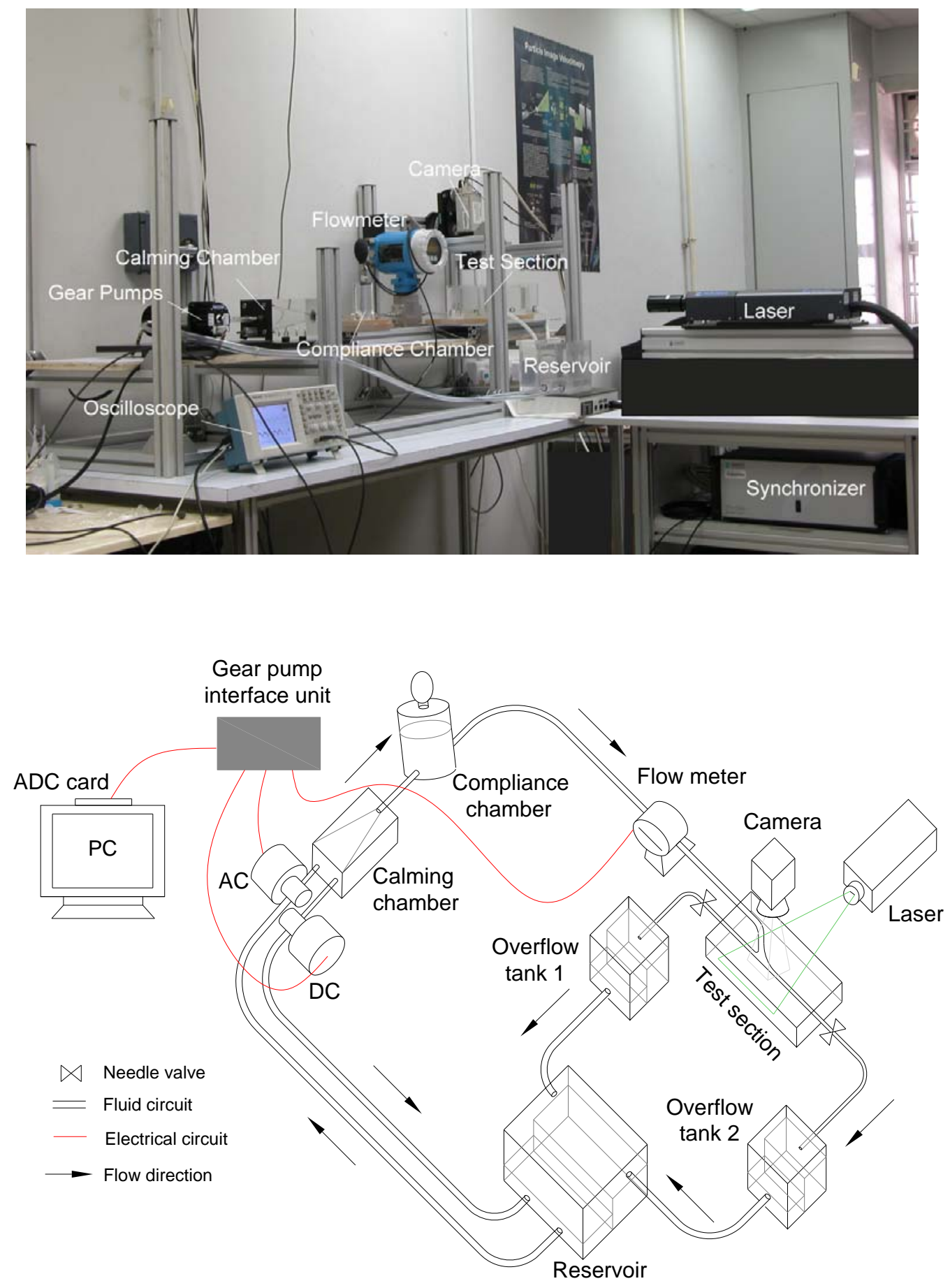

Fig. 7.3 System picture (top) and diagram (bottom) of the flow circuit. 

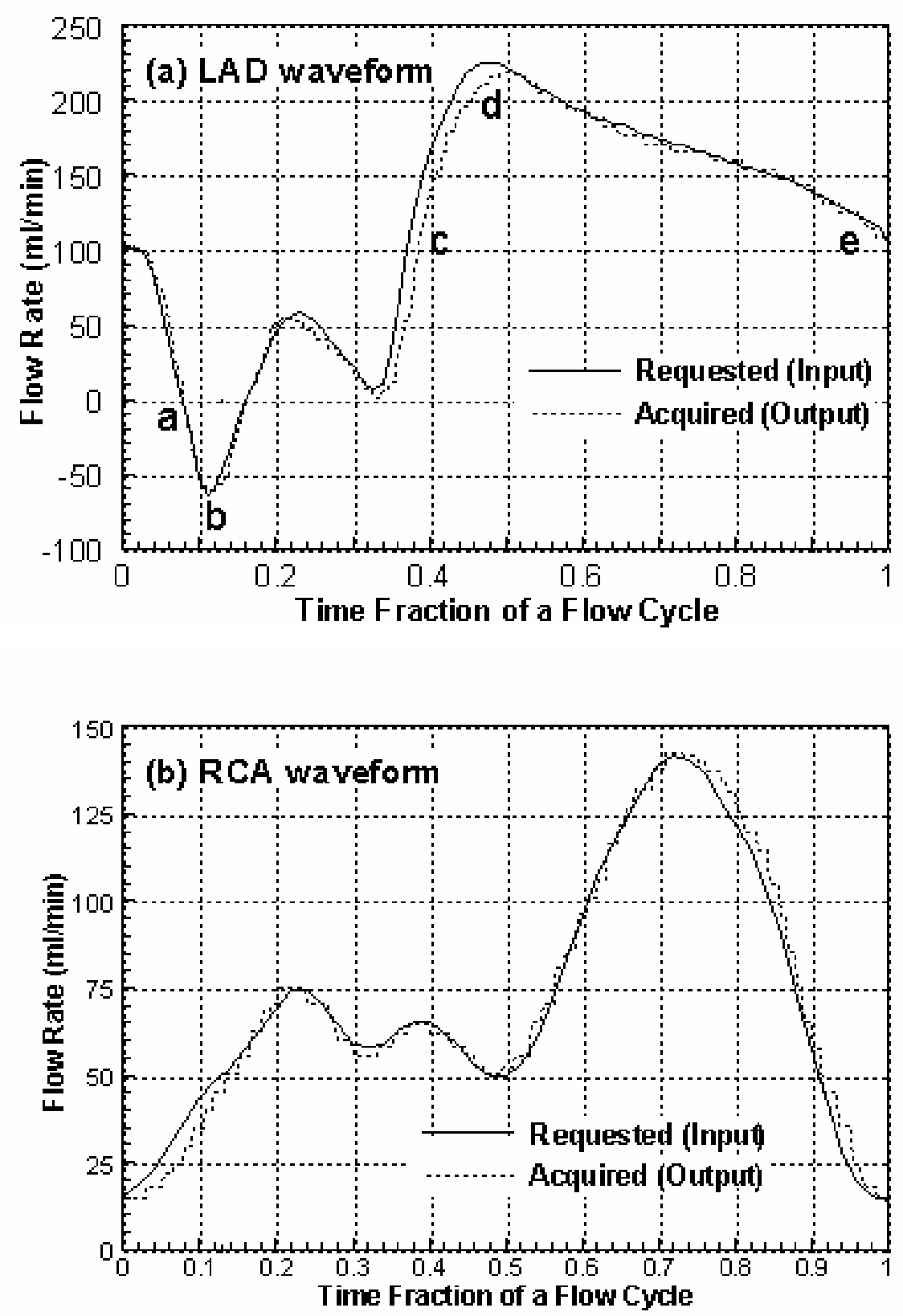

Fig. 7.4 Experimental and numerical inlet flow waveforms.

\subsubsection{Working Fluid and Tracer Particles}

The working fluid was an aqueous solution of glycerol ( $42 \%$ by weight), which provided a dynamic viscosity of about $4 \mathrm{mPa} s$ and density of $1.1 \mathrm{~g} / \mathrm{cm}^{3}$, comparable to the blood at a temperature of $25^{\circ} \mathrm{C}$ (Heise et al., 2004). Hollow glass spheres with a mean size of $10 \mu \mathrm{m}$ and density of $1.1 \mathrm{~g} / \mathrm{cm}^{3}$ (HGS-10, Dantec Dynamics, Denmark) were used as tracer particles and seeded into the fluid. 
Seeding quality in the PIV measurement is crucial to successful acquisition of experimental data. First, the particles should be small enough to track the flow accurately, yet large enough to scatter sufficient light for the camera to be able to detect them. Generally, the particle size decreases with increasing flow velocity, turbulence and velocity gradient. The shape of the particles is also important as it affects the drag force exerted on them by the surrounding fluid. Second, the particles should have less variation in size and should be neutrally buoyant in the fluid to avoid particle clogging and adhesion to the inner surface; that is, they should have approximately the same density as the fluid. In this experiment, hollow glass spherical particles of $10 \mu \mathrm{m}$ in diameter with a mean density of $1.1 \mathrm{~g} / \mathrm{cm}^{3}$ proved to be an ideal choice. Before seeding the particles were specially treated using the following procedure: dissolve the particles in the glycerol solution, stir them evenly and leave for a sufficient time. This way, the particles that were either heavier or lighter than the fluid were separated and discarded.

\subsubsection{The PIV System}

The PIV is capable of providing accurate and high quality measurements of instantaneous global flow fields in a variety of laboratory-scale flows. In PIV the fluid velocity is determined by measuring the distance traveled by tracer particles in the flow within a known time interval (Adrian, 1991). A complete planar PIV system includes a laser, light sheet optics, a camera and a synchronizer. The detailed descriptions of the experimental apparatus are provided in Appendices B.

Particles in an area of the flow field were illuminated by a light sheet generated by the laser and the system of optical components. The light sheet was pulsed to produce a stroboscopic effect, freezing the movement of the seeding particles. The particles scattered light into a photographic lens of the camera, which was positioned 
perpendicular to the light-sheet to ensure that its in-focus object plane coincides with the illuminated slice of fluid. The pulsing light-sheet and the camera were synchronized via the synchronizer so that the camera can instantly capture the images of particle positions at each light pulse. The laser used in the present study was a Q-switched, dual-head pulsed Nd:YAG laser (Gemini PIV 200-15, New Wave Research, Inc., USA) with a maximum energy of $200 \mathrm{~mJ}$ at a wavelength of 532nm and a repetition rate of 15 HZ. An 80C77 HiSense MkII camera with a Nikon Micro-Nikkor lens 60/f2.8 was used to detect the position of the illuminated particles. Two images were recorded in rapid succession by the illumination of the first and second pulses of the light sheet with a time interval or pulse separation $\Delta t=0.1 \sim 1.2 \mathrm{~ms}$ depending on the transient velocity magnitude at different time steps of the flow cycle. The choice of $\Delta t$ is crucial for the success of PIV measurement, the estimation of which will be discussed later. The camera images were divided into rectangular regions called interrogation areas. For each of these interrogation areas the two images were correlated to produce a vector map of average particle displacements. The displacement vectors were then converted into a map of raw velocity vectors by dividing with $\Delta t$.

\subsubsection{Parameters in PIV Measurement}

A typical planar PIV optical system is illustrated in Fig. 7.5. For the lens of focal length $f$, the object distance $d_{o}$, the image distance $d_{i}$ and the image-object scale factor $\mathrm{S}$ have the following relationship (Keane and Adrian, 1990):

$$
\begin{aligned}
& d_{o}=\left(1+S^{-1}\right) f \\
& d_{i}=(1+S) f \\
& S=d_{i} / d_{o}
\end{aligned}
$$


The scale factor was estimated from an image of a ruler placed in the light sheet plane prior to the PIV measurements. The scale factor in the present study was 1.513 .

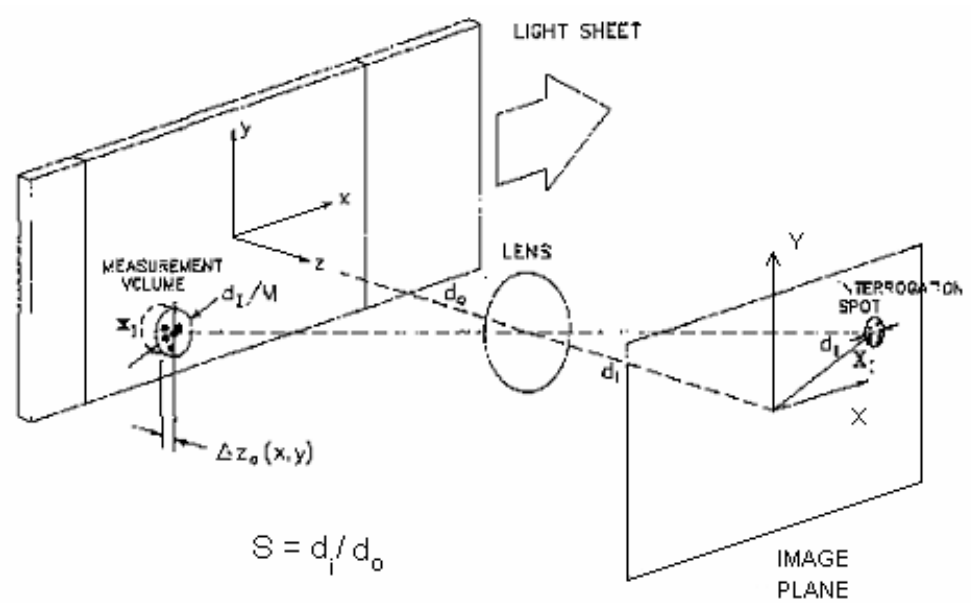

Fig. 7.5 Optical system of planar pulsed particle image velocimetry (from Keane and Adrian, 1990, with permission).

For typical recording situations, the diameter of a particle image is not simply $S d_{p}$ where $d_{p}$ is the particle diameter in the object plane. As a result of diffraction effect, a point source in the object plane at a position O' will spread out into an Airy function on the image plane centered at $\mathrm{O}$ ' as shown in Fig. 7.6. The point response function is used to describe the diffraction effect, which is an Airy function of diameter

$$
d_{s} \approx 2.4(1+S) \frac{f}{D} \lambda
$$

where $\mathrm{D}$ is the diameter of the camera aperture, the ratio $f / D$ is the f-number of the lens, and $\lambda$ is the wavelength of the light. Finally, the image diameter can be estimated by the formula (Prasad, 2000):

$$
d_{e} \approx \sqrt{S^{2} d_{p}^{2}+d_{s}^{2}}
$$

Using $f / D=8, \lambda=532 \mathrm{~nm}, \mathrm{~S}=1.513$ and $d_{p}=10 \mu \mathrm{m}$ yields to $d_{e}=30.08 \mu \mathrm{m}$, which is large enough to exceed the pixel dimensions as required in the measurements. 


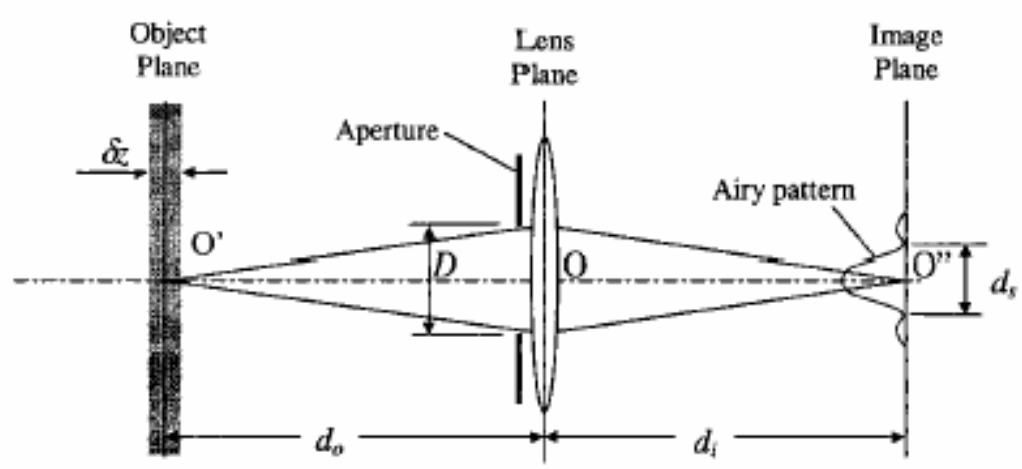

Fig. 7.6 Diffraction effects during particle image recording (from Prasad, 2000, with permission).

Another critical parameter affecting the quality of particle images is the depth-of-field $\delta z$, which is the thickness of the region over which particles will be in acceptable focus (Prasad, 2000):

$$
\delta z \approx 4\left(1+S^{-1}\right)^{2}(f / D)^{2} \lambda
$$

The $\delta z$ increases rapidly with increasing the f-number and decreasing the scale factor S. For the values used above, the $\delta z$ is calculated to be $0.7 \mathrm{~mm}$. That is to say, the thickness of the light sheet should not be larger than $0.7 \mathrm{~mm}$ to avoid imaging out-offocus particles.

The delay $\delta t$ (or duration) and separation $\Delta t$ of pulses for a PIV system are illustrated in Fig. 7.7. The pulse delay usually determines the degree to which an image is frozen during the pulse exposure. The pulsed laser used has a short delay of less than 10 nanoseconds, which is short enough to freeze the particle motion at even high speeds with minimal blurring. The pulse separation $\Delta t$ is the single most important adjustable variable in the PIV measurement. An appropriate $\Delta t$ between exposures can be roughly estimated by knowing the expected flow velocity in the object plane, the scale factor, and the interrogation area size in the image plane according to Keane and Adrian (1990). To achieve a high detection rate the maximum particle displacement $\left(d_{\max }\right)$ between 
successive images should be less than $1 / 4$ of the size of the interrogation area $\left(l_{\text {int }}\right)$, e.g. 8 pixels for a $32 \times 32$ pixel area. This can be expressed as

$$
d_{\max }=u_{\max } \cdot \Delta t \cdot S \leq 0.25 l_{\mathrm{int}}
$$

where $u_{\max }$ is the estimated maximum velocity at a specific time step in the object plane.

Then the pulse separation between a pair of images should meet the following criterion

$$
\Delta t \leq \frac{0.25 l_{\text {int }}}{u_{\max } S}
$$

The minimum measurable particle displacement $\left(d_{\min }\right)$ is smaller than 1 pixel when cross-correlation is used. That is to say, the cross-correlation method can allow $\Delta t<$ $\frac{d_{e}}{u_{\min } S}$. Therefore, based on the estimation criteria of $\Delta t$, the time delay between two consecutive pulses in the present study was ranged from $0.1 \mathrm{~ms}$ to $1.2 \mathrm{~ms}$ depending on the model size and mean flow velocity at different time steps.

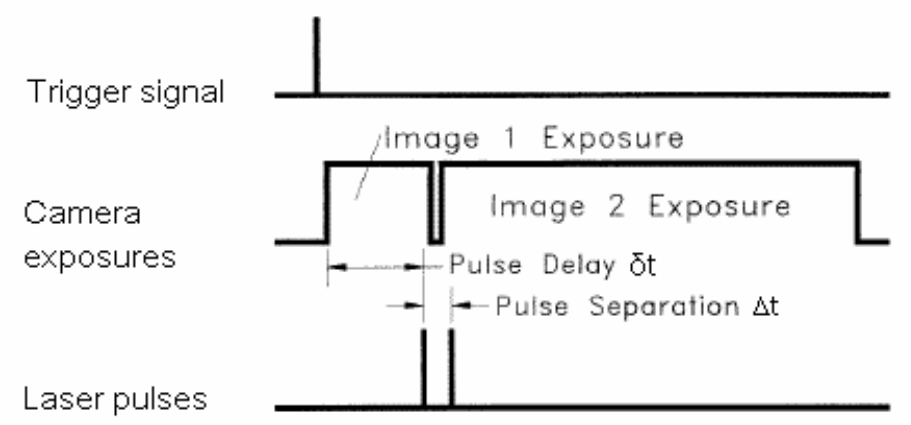

Fig. 7.7 Pulse delay and pulse separation in double video frames.

\subsubsection{Data Acquisition and Processing}

The PIV image acquisition was triggered by TTL signals generated by the gear pump system, and data were acquired at 25 discrete time steps of the flow cycle. For each time step at least 30 valid measurements were obtained. A measurement was considered acceptable if the calculated raw vector map provided meaningful information and the 
pulsatility index, frequency and mean velocity of the corresponding waveform were close to the requested values. The flow waveform was monitored and analyzed in real time on the computer through an oscilloscope-based program while acquiring the images.

The PIV recordings from the camera were interrogated using the PIV software package FlowManager (Dantec Dynamics, Denmark). For the images, cross-correlation analyses based on Fast Fourier Transform algorithms were used with interrogation areas of $32 \times 32$ pixels. The interrogation areas were overlapped by $25 \%$ at each of their sides. Overlapping interrogation areas was to optimize the use of information in the image map. For each time step, at least 30 recordings of image data were used to give an averaged vector field. The presence of background optical noises introduced a small number of spurious vectors called outliers. The vector fields were therefore validated using peak-height validation, velocity range validation and moving-average validation methods. The peak-height validation was to reject individual false vectors based on the height ratio between the highest peak (signal) and the second highest peak (noise) in the correlation plane. In this study, a vector was rejected if the signal-to-noise ratio is less than 1.2 (Keane and Adrian, 1992). The velocity range validation was to remove vectors that fall outside the predefined thresholds for the vector continuity and velocity magnitude. The thresholds were usually the estimated maximum and minimum velocities at the corresponding time step. Finally, the moving-average validation, which is based on an implicit assumption of continuity of the flow field's behaviour, was used to reject vectors which are not continuous with the neighbouring vectors, and substitute for them as well as previously rejected vectors with averaged vectors estimated from surrounding values. The averaging area was set to $3 \times 3$ pixels, and the acceptance factor 
was specified to be 0.1 . Since only a few outliers existed, a single iteration was enough to remove all the deviating vectors.

The velocity data were presented using velocity-magnitude contours superimposed by in-plane velocity vectors at typical time steps of the flow cycle. Instantaneous WSS $(\tau)$ on the floor and ceiling of the model was derived using the partial derivatives of the velocity components with respect to both $\mathrm{x}$ and $\mathrm{y}$ :

$$
\tau=\mu\left(\frac{\partial V_{y}}{\partial x}+\frac{\partial V_{x}}{\partial y}\right)
$$

Here $\mu$ is the dynamic viscosity of the blood, and $V_{x}$ and $V_{y}$ are the $\mathrm{x}$ and y velocity components, respectively.

\subsubsection{Uncertainty and Error Analysis}

Two types of errors exist in the PIV measurements: systematic and random errors. Systematic errors are mainly caused by the uncertainty in the determination of the geometric parameters and the fabrication tolerances of the measuring devices such as the camera and lenses (Raffle et al., 1998). In the present study, the systematic errors were generally less than $1 \%$ for the full range of calculated velocities. The random errors produced the largest uncertainties associated with the determination of the average particle displacement in the interrogation region (Christensen and Adrian, 2002). These errors depend primarily on the time interval between two successive pulses $\Delta t$, the calibrated scale factor $\mathrm{S}$, and the velocity gradient of the flow $\Delta u$. The error of the scale factor was typically one pixel in 1000 , i.e. $\delta(S)=0.1 \%$. The pulse interval has a maximum deviation of $5 \mathrm{rpm}$ in $1000 \mathrm{rpm}$, i.e. $\delta(\Delta t)=0.5 \%$. The most important source of the random error is the presence of high spatial velocity gradients in certain regions. A large local velocity gradient can lead to unequal particle displacements causing the correlation to be biased against the faster moving particles 
within the measurement volume. The maximum random error in velocity occurs in the highest shear region and is estimated within $5 \%$.

In order to quantify the agreement or discrepancy between the simulation and the PIV experiment, the numerically predicted WSS was interpolated to the PIV calculation points and two sets of data were directly compared using two methods of error estimation. The first method was to use a percentage relative error $\left(\varepsilon_{r}\right)$, which is defined as

$$
\varepsilon_{r}=\left|\left(\tau_{\text {num }}-\tau_{\text {exp }}\right) / \tau_{\text {exp }}\right| \times 100 \%
$$

Then, the average relative error was calculated as

$$
E_{r}=\frac{1}{n} \sum_{i=1}^{n} \varepsilon_{r}(i)
$$

Here $i$ is the data point number, $\varepsilon_{r}(i)$ is the relative error at the individual data point $i$, and the sum was calculated over the transient errors of $n$ PIV measurements over the flow cycle. A normalized error was estimated as

$$
\varepsilon_{\text {norm }}=\left(\left(\tau_{\text {num }}-\tau_{\text {exp }}\right) \mid / \tau_{\max }\right) \times 100 \%
$$

and the average absolute error was calculated as

$$
E_{\text {norm }}=\frac{1}{n} \sum_{i=1}^{n} \varepsilon_{\text {norm }}(i)
$$

where $\tau_{\max }=\frac{8 \mu^{2} \operatorname{Re}_{\max }}{\rho d_{a}{ }^{2}}$, is the Poiseuille-type WSS in the straight native artery at the maximum Reynolds number. 


\subsection{RESULTS: LAD MODEL}

Figure 7.8 shows the velocity-magnitude contours superimposed by in-plane velocity vectors in the $\mathrm{x}-\mathrm{y}$ symmetry plane of the modified model at five typical time steps of the flow cycle by both numerical modeling and PIV measurement. There is good agreement for velocity fields at $\mathrm{t}=0.12,0.40$, and 0.96 cycle except a lower resolution in the experimental data. Relatively larger discrepancies occur at $\mathrm{t}=0.08$ and 0.48 cycle, particularly in the outflow vessel. At $t=0.08$ cycle when the external pressure has just transited to negative, the low-momentum fluid near the graft wall that is more easily driven by the external pressure becomes already retrograde, whereas the higher-inertia fluid in the mainstream have not decayed from the previous part of the cardiac cycle and remains forward. The main flow features, such as the retrograde flow near the ceiling, forward flow in the mainstream, as well as a clockwise rotating vortex structure in the proximal part of the model, are demonstrated in both flow fields. Closer examination, however, reveals that the experimental data has stronger retrograde flow along the ceiling of the distal outlet segment and weaker forward flow in the center of the vessel. The difference between the numerical and experimental flow fields is also appreciable at the peak diastolic flow. It is observed that the experimental data have a smaller flow volume in the distal outlet segment but a larger volume in the proximal segment as compared to the numerical case. The whole flow profile in the experimental case seems to be dragged slightly towards the proximal direction, resulting in a more extensive low flow area along the ceiling of the graft.

Figure 7.9 compares the numerical and experimental WSS variations over the flow cycle at selected points of the anastomotic region. The maximum and averaged values of both relative and normalized errors are tabulated (Table 7.1). The WSS curves of the two upstream points $\mathrm{X}=0$ and $0.5 d_{a}$ on the ceiling give the best match between the 
numerical and experimental results, showing average relative errors $\left(E_{r}\right)$ of $7.7 \%$ and $7.9 \%$, respectively, and average normalized errors $\left(E_{\text {norm }}\right)$ of only $1.0 \%$ and $0.8 \%$, respectively. Errors are apparently increased at the downstream points $\mathrm{X}=1.0,1.5$, and $2.0 d_{a}$ on the ceiling, ranging between $14.9 \sim 19.2 \%$ in relative error and $1.3 \sim 4.2 \%$ in normalized error. The points on the artery floor generally have larger errors than those on the ceiling, with the largest average relative errors between $20.2 \sim 28.4 \%$ and the largest average normalized errors between $2.0 \sim 8.9 \%$. The transient relative errors are more variable, with the maximum $\left(\varepsilon_{r}\right)$ up to $85.4 \%$, as compared to the transient normalized errors $\left(\varepsilon_{n o r m}\right)$. Note that larger relative errors always occur when the experimental WSS has extremely low magnitudes. In these cases, the corresponding normalized errors are usually small. For example, the largest relative error of $85.4 \%$, which is found at $X=-0.5 d_{a}$ and $t=0.12$ cycle, corresponds to a normalized error of only $1.6 \%$ (not tabulated). In contrast, larger normalized errors always occur at larger WSS magnitudes, e.g. the largest error of $21.3 \%$ is found at $X=2.0 d_{a}$ on the floor around the peak diastolic flow. The average magnitudes of all normalized errors are kept below $10 \%$.

The WSS variations along the floor at selected time steps for the numerical and experimental results are compared in Fig. 7.10, and the relative and normalized errors are displayed in Tab. 7.2. Both the smallest relative error (3.6 3.7\%) and normalized error $(2.4 \sim 3.1 \%)$ occur at the time steps $t=0.40$ and 0.96 of the cycle with Reynolds numbers corresponding to 266.8 and 230, respectively. Large errors, however, are shown at $\mathrm{t}=0.16,0.48$ and 0.76 with Reynolds numbers of $-13.4,429.6$ and 318.5, respectively. The comparison at $\mathrm{t}=0.48$ give both the largest relative error of $23.5 \%$ and the largest normalized error of $8.8 \%$. Generally, the higher the deviation from the 
mean Reynolds number, the larger is the discrepancy between the numerical and experimental curves. Again, larger normalized errors are usually associated with the curves with larger WSS magnitudes during the high-flow phases. For all of the curves the general trend of the experimental WSS variations conforms well to the numerical results.
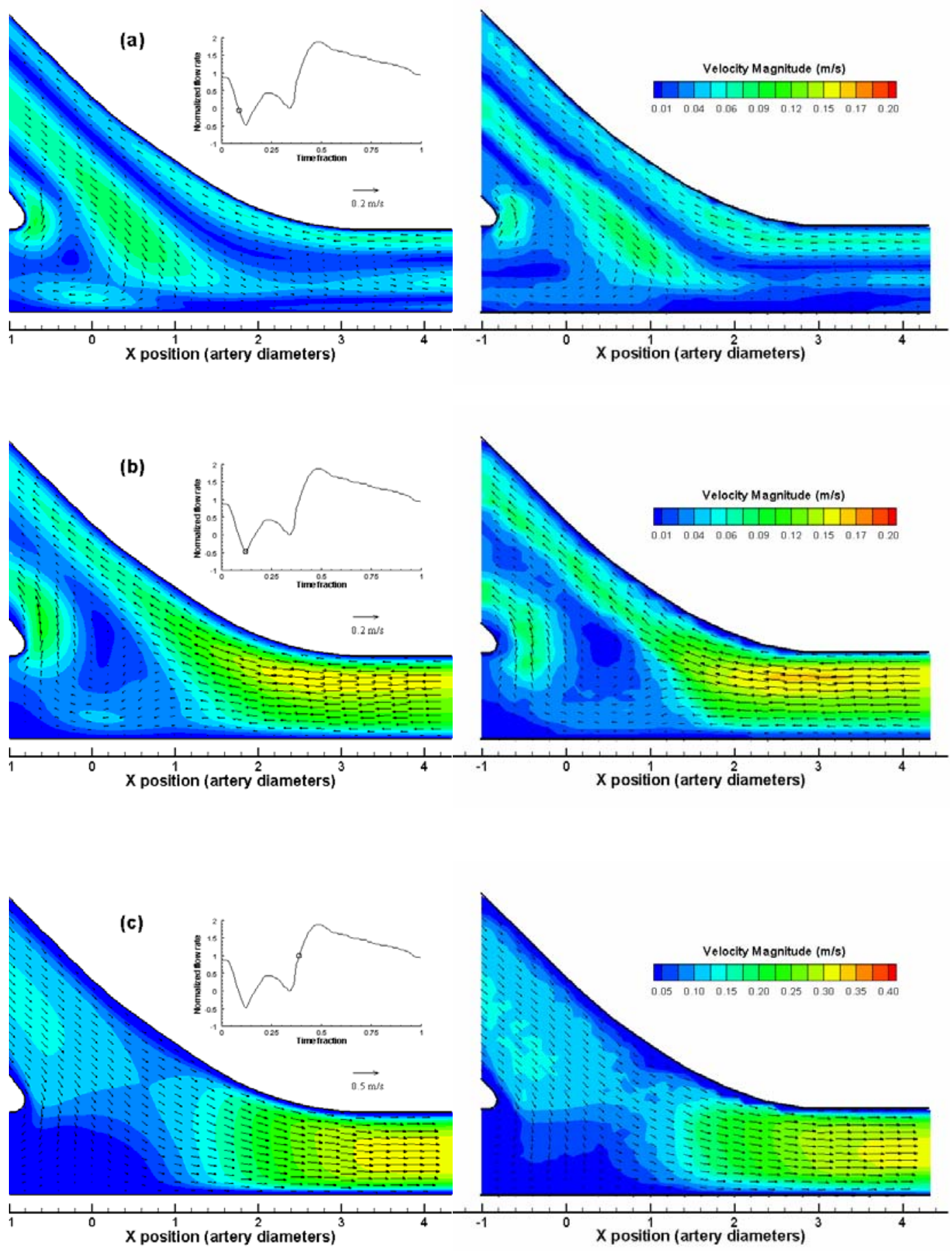

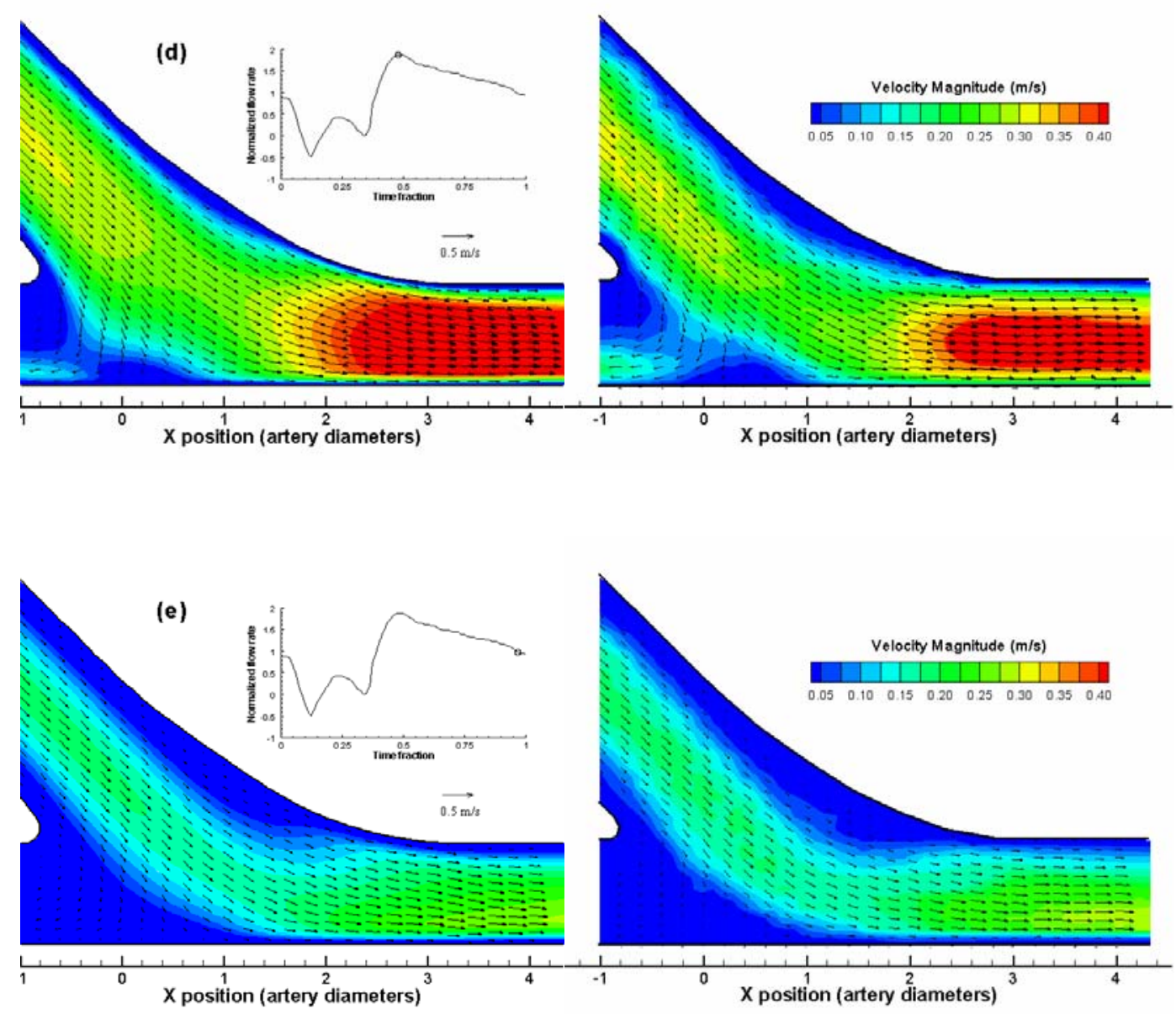

Fig. 7.8 Comparisons of transient flow fields between numerical simulation (left) and experimental measurement (right) for the LAD model: (a) $t=0.08$ cycle, (b) $t=0.12$ cycle, (c) $\mathrm{t}=0.40$ cycle, (d) $\mathrm{t}=0.48$ cycle, and (e) $\mathrm{t}=0.96$ cycle.

(a)

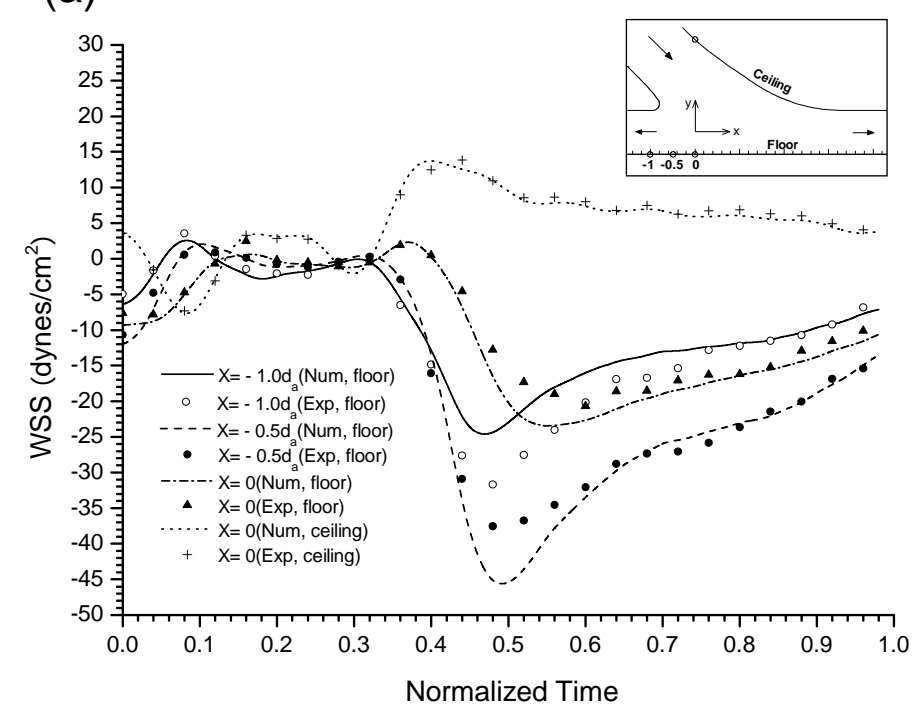


(b)

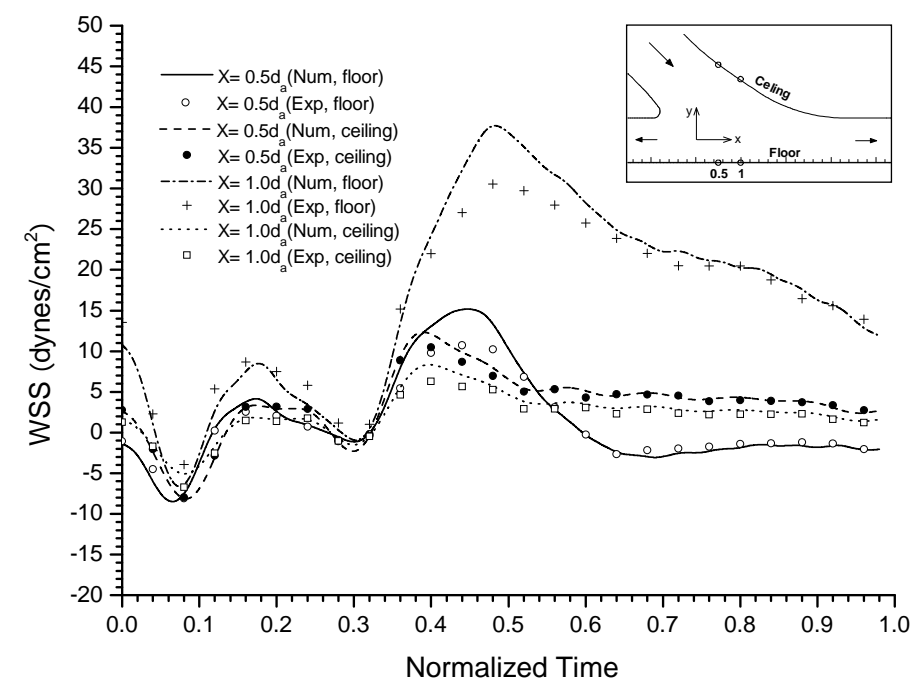

(c)

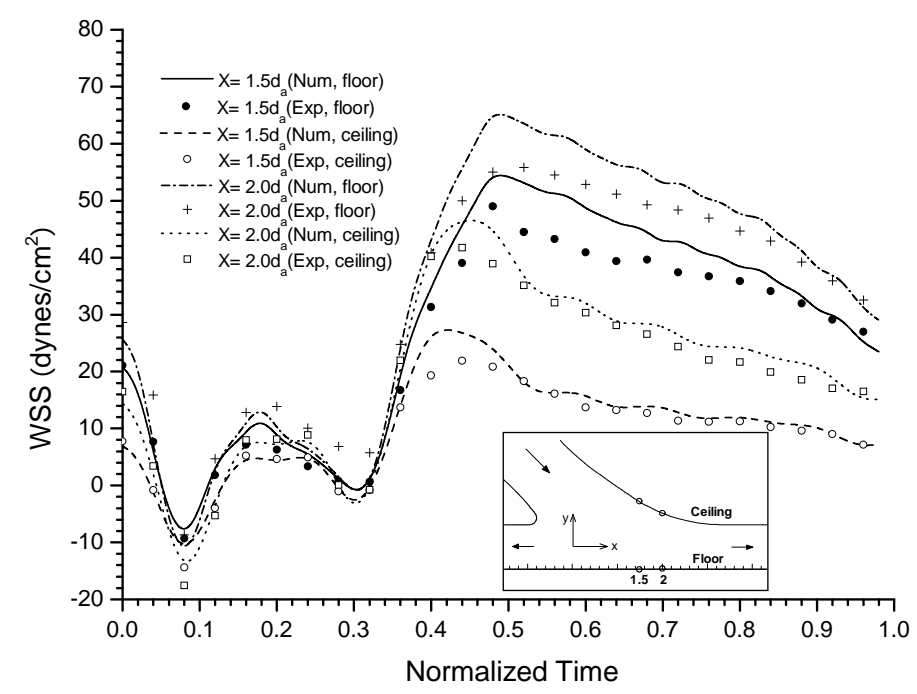

Fig. 7.9 Comparisons of WSS variations over the flow cycle at selected points of the anastomotic region between numerical simulation and experimental measurement for the LAD model. 
Table 7.1 Summary of relative and normalized errors for the comparisons of the numerical and PIV WSS variations over the flow cycle at selected points.

\begin{tabular}{lcccc}
\hline Selected Points & Maximum & Average & Maximum & \multicolumn{2}{c}{ Average } \\
& $\varepsilon_{r}(\%)$ & $E_{r}(\%)$ & $\varepsilon_{\text {norm }}(\%)$ & $E_{\text {norm }}(\%)$ \\
\hline $\mathrm{X}=-1.0 \mathrm{~d}_{\mathrm{a}}$, floor & 82.2 & 22.8 & 15.9 & 4.2 \\
$\mathrm{X}=-0.5 \mathrm{~d}_{\mathrm{a}}$, floor & 85.4 & 23.3 & 14.8 & 3.3 \\
$\mathrm{X}=0$, floor & 77.3 & 22.7 & 11.1 & 3.0 \\
$\mathrm{X}=0$, ceiling & 14.7 & 7.7 & 2.8 & 1.0 \\
$\mathrm{X}=0.5 \mathrm{~d}_{\mathrm{a}}$, floor & 75.8 & 28.4 & 9.5 & 2.0 \\
$\mathrm{X}=0.5 \mathrm{~d}_{\mathrm{a}}$, ceiling & 16.3 & 7.9 & 3.5 & 0.8 \\
$\mathrm{X}=1.0 \mathrm{~d}_{\mathrm{a}}$, floor & 74.9 & 21.6 & 15.6 & 4.4 \\
$\mathrm{X}=1.0 \mathrm{~d}_{\mathrm{a}}$, ceiling & 34.9 & 19.2 & 4.4 & 1.3 \\
$\mathrm{X}=1.5 \mathrm{~d}_{\mathrm{a}}$, floor & 65.4 & 21.4 & 18.9 & 7.3 \\
$\mathrm{X}=1.5 \mathrm{~d}_{\mathrm{a}}$, ceiling & 53.2 & 15.9 & 14.8 & 2.8 \\
$\mathrm{X}=2.0 \mathrm{~d}_{\mathrm{a}}$, floor & 78.2 & 20.2 & 21.3 & 8.9 \\
$\mathrm{X}=2.0 \mathrm{~d}_{\mathrm{a}}$, ceiling & 54.4 & 14.9 & 12.6 & 4.2 \\
\hline
\end{tabular}

Table 7.2 Summary of relative and normalized errors for the comparisons of the numerical and PIV WSS variations along the floor at selected time steps.

\begin{tabular}{ccc}
\hline Time steps & $\begin{array}{c}\text { Average } \\
E_{r}(\%)\end{array}$ & $\begin{array}{c}\text { Average } \\
E_{\text {norm }}(\%)\end{array}$ \\
\hline $\mathrm{t}=0.12$ & 20.0 & 2.7 \\
$\mathrm{t}=0.16$ & 22.1 & 5.6 \\
$\mathrm{t}=0.24$ & 18.6 & 3.1 \\
$\mathrm{t}=0.40$ & 3.7 & 3.1 \\
$\mathrm{t}=0.48$ & 23.5 & 8.8 \\
$\mathrm{t}=0.76$ & 19.8 & 6.2 \\
$\mathrm{t}=0.96$ & 3.6 & 2.4 \\
\hline
\end{tabular}


(a)

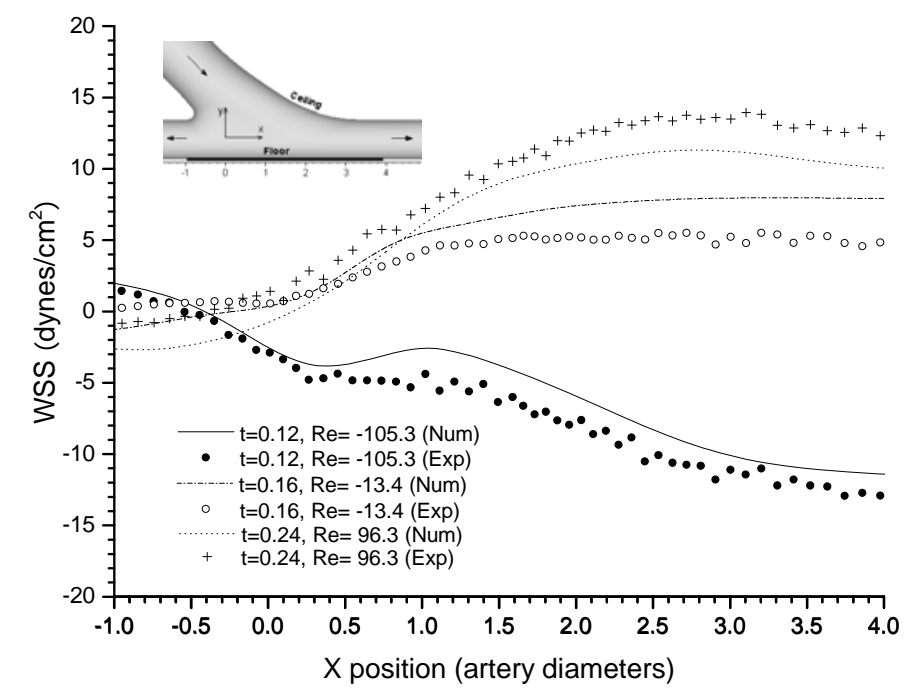

(b)

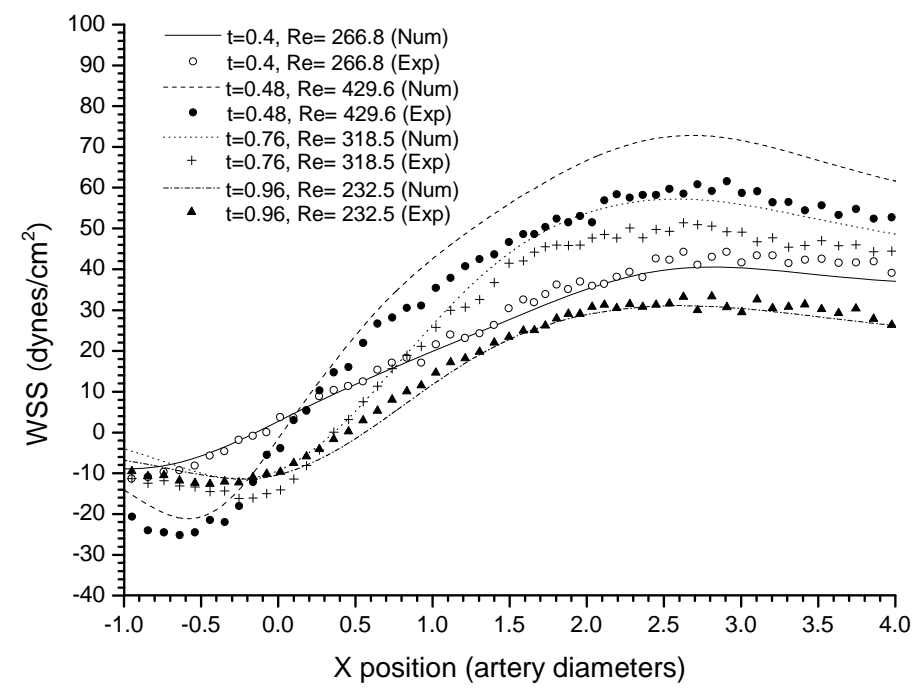

Fig. 7.10 Comparisons of WSS variations along the floor at selected time steps for the LAD model. 


\subsection{RESULTS: RCA MODEL}

The RCA waveform, which contains no negative flow phase, produces less complex flow fields as compared to the LAD waveform, and discrepancies between the experimental and numerical results are also shown to be smaller. Flow patterns are examined at five time steps representing the peak systole $(t=0.24$ cycle $)$, diastolic acceleration $(t=0.60$ cycle $)$, peak diastole $(t=0.72$ cycle $)$, mid diastolic deceleration $(t$ $=0.88$ cycle $)$, and late diastolic deceleration $(t=0.96$ cycle $)$ as shown in Fig. 7.11. The numerical and experimental flow fields have an excellent match at $\mathrm{t}=0.24,0.60$ and 0.88 cycle, showing comparable velocity magnitudes in both the inlet and outlet segments. The areas of stagnant flow on the floor and in the vicinity of the heel are also similar. At the peak flow $t=0.72$ cycle, however, more flow seems to be driven into the proximal outlet segment in the experimental flow field, causing the low flow area just proximal to the heel to be reduced in size. Contrarily, the numerical flow field exhibits higher flow in the distal outlet segment. Differences between the two flow fields are also apparent at $\mathrm{t}=0.96$ cycle when the flow is nearly the minimum. Higher distal outflow and lower proximal flow are associated with the experimental results.

Figure 7.12 compares the WSS variations over the flow cycle at selected points of the anastomotic region between the numerical and experimental results, and the relative and normalized errors for each pair of curves are calculated and displayed in Tab. 7.3. Similar to the results for the LAD model, the smallest difference is associated with the curves of the upstream point $\mathrm{X}=0.5 d_{a}$ on the ceiling, corresponding to an average relative error of $6.8 \%$ and an average normalized error of $1.6 \%$. The largest normalized error (5.2 5.4\%) appears at the points $\mathrm{X}=2 d_{a}$ and $3 d_{a}$ on the floor, which have the highest WSS levels as a result of impinging flow effect, particularly at the peak flow 
when the flow velocity is highest. The largest relative error, being $21.7 \%$, occurs at $\mathrm{X}=$ $0.5 d_{a}$ on the floor, whereas the corresponding normalized error is only $2.3 \%$. This is the common location of flow division point and has an extremely low WSS level. In addition, for all the distal points of the model, the experimental WSS magnitudes are smaller during the high flow phases near the peak flow but larger during the low flow phases than is observed in the numerical results; the situation is otherwise for the proximal points.
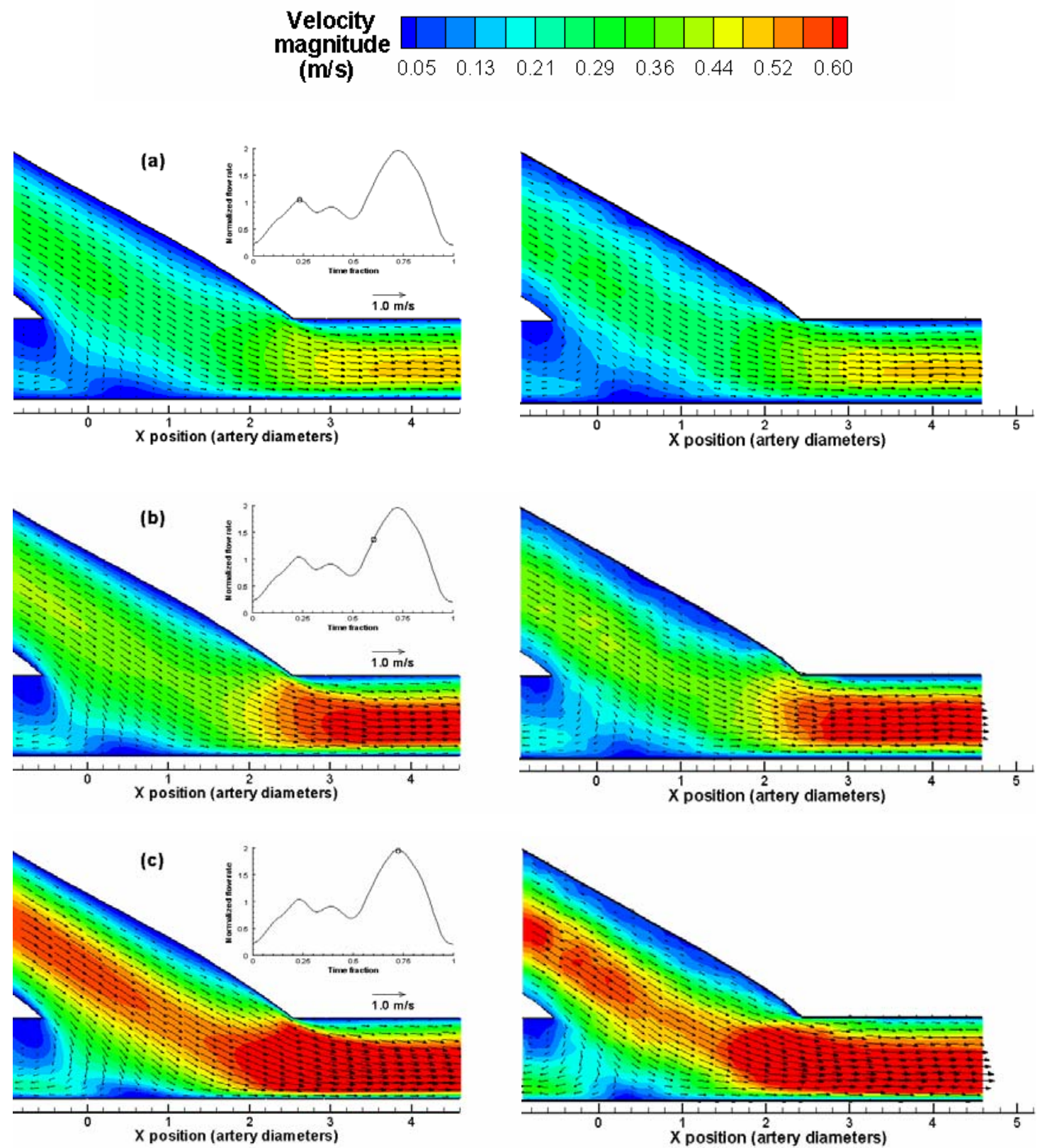

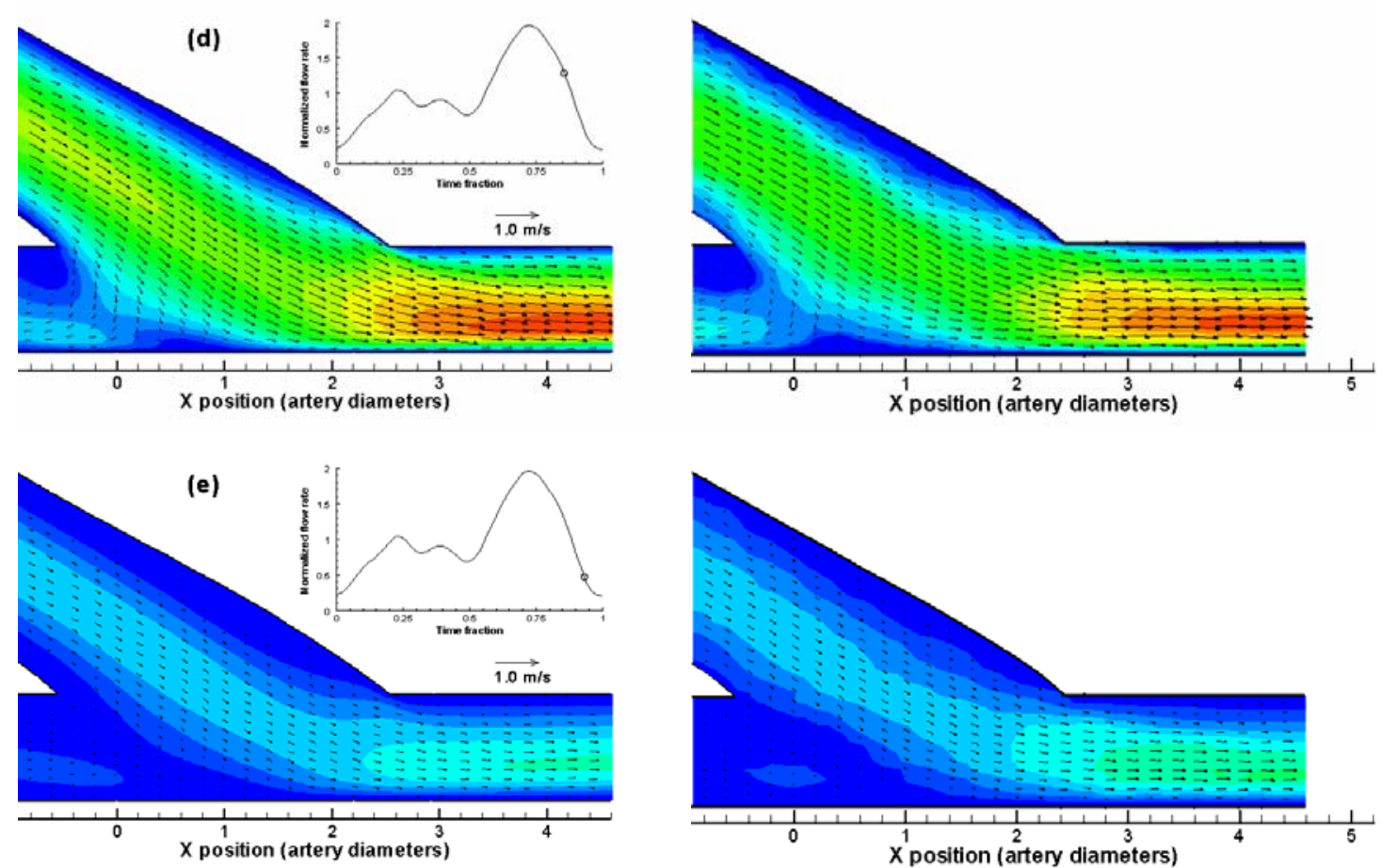

Fig. 7.11 Comparisons of transient flow fields between numerical simulation (left) and experimental measurement (right) for the RCA model: (a) $t=0.24$ cycle, (b) $t=0.60$ cycle, (c) $\mathrm{t}=0.72$ cycle, (d) $\mathrm{t}=0.88$ cycle, and (e) $\mathrm{t}=0.96$ cycle.

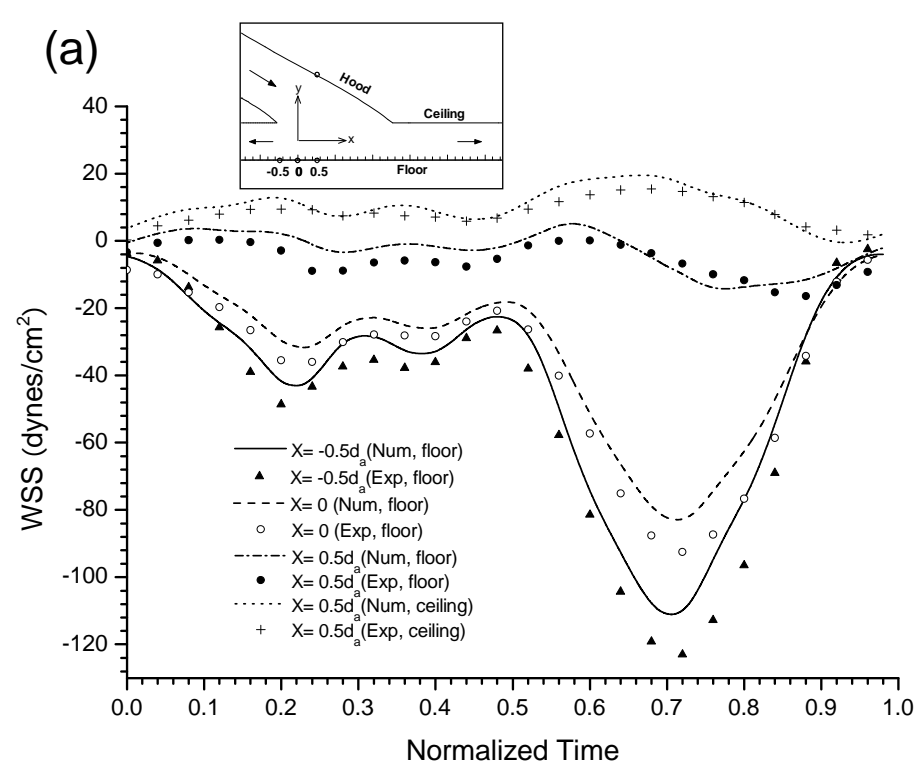




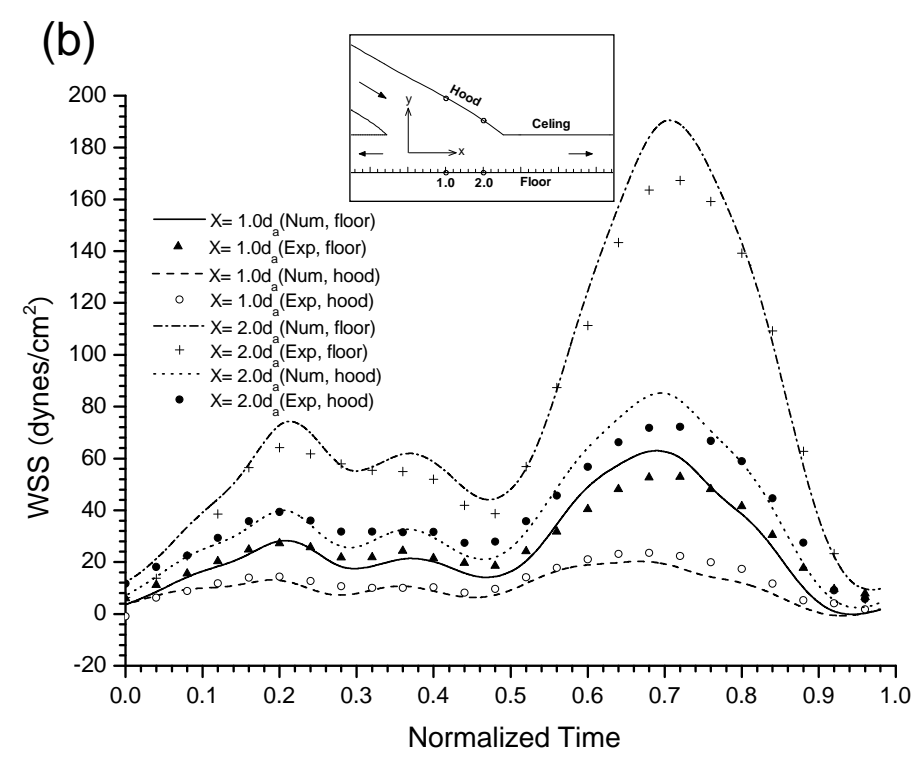

(c)

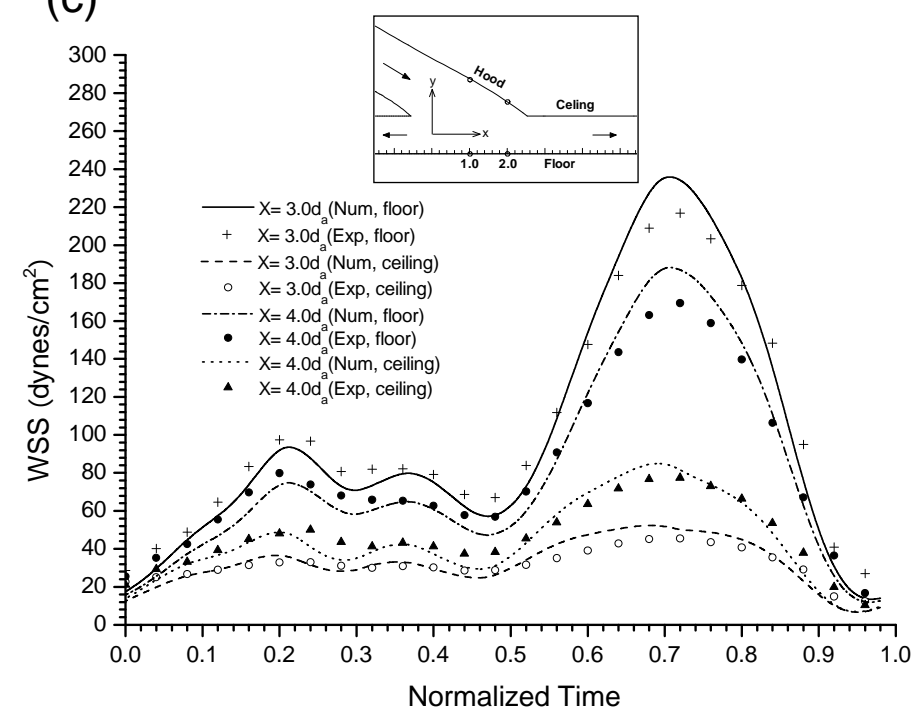

Fig. 7.12 Comparisons of WSS variations over the flow cycle at selected points of the anastomotic region between numerical simulation and experimental measurement for the RCA model. 


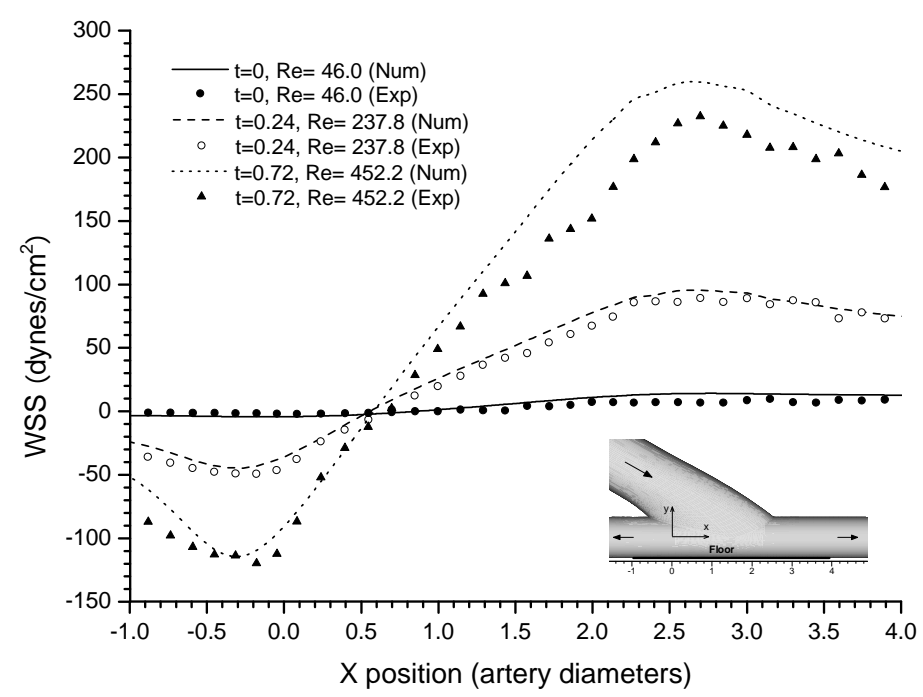

Fig. 7.13 Comparisons of WSS variations along the floor at selected time steps for the RCA model.

Table 7.3 Summary of relative and normalized errors for the comparisons of the numerical and PIV WSS variations over the flow cycle at selected points.

\begin{tabular}{lcccc}
\hline $\begin{array}{l}\text { Positions } \\
\text { on the model }\end{array}$ & $\begin{array}{c}\text { Maximum } \\
\varepsilon_{r}(\%)\end{array}$ & $\begin{array}{c}\text { Average } \\
E_{r}(\%)\end{array}$ & $\begin{array}{c}\text { Maximum } \\
\varepsilon_{\text {norm }}(\%)\end{array}$ & $\begin{array}{c}\text { Average } \\
E_{\text {norm }}(\%)\end{array}$ \\
\hline $\mathrm{X}=-0.5 \mathrm{~d}_{\mathrm{a}}$, floor & 83.3 & 18.8 & 19.1 & 4.3 \\
$\mathrm{X}=0$, floor & 65.2 & 17.3 & 11.9 & 3.8 \\
$\mathrm{X}=0.5 \mathrm{~d}_{\mathrm{a}}$, floor & 76.8 & 21.7 & 6.3 & 2.3 \\
$\mathrm{X}=0.5 \mathrm{~d}_{\mathrm{a}}$, ceiling & 34.5 & 6.8 & 4.0 & 1.6 \\
$\mathrm{X}=1.0 \mathrm{~d}_{\mathrm{a}}$, floor & 66.0 & 15.8 & 10.6 & 3.4 \\
$\mathrm{X}=1.0 \mathrm{~d}_{\mathrm{a}}$, ceiling & 60.1 & 9.9 & 4.8 & 1.8 \\
$\mathrm{X}=2.0 \mathrm{~d}_{\mathrm{a}}$, floor & 72.7 & 11.9 & 20.0 & 5.4 \\
$\mathrm{X}=2.0 \mathrm{~d}_{\mathrm{a}}$, ceiling & 65.0 & 8.8 & 10.8 & 2.8 \\
$\mathrm{X}=3.0 \mathrm{~d}_{\mathrm{a}}$, floor & 52.9 & 12.8 & 13.9 & 5.2 \\
$\mathrm{X}=3.0 \mathrm{~d}_{\mathrm{a}}$, ceiling & 40.0 & 11.8 & 6.3 & 2.0 \\
$\mathrm{X}=4.0 \mathrm{~d}_{\mathrm{a}}$, floor & 38.6 & 11.3 & 13.5 & 4.0 \\
$\mathrm{X}=4.0 \mathrm{~d}_{\mathrm{a}}$, ceiling & 30.2 & 8.0 & 8.7 & 3.0 \\
\hline
\end{tabular}




\subsection{DISCUSSION}

The use of PIV measurement to complement numerical modeling to quantify coronary anastomotic hemodynamics has been explored. Here, PIV data is used to validate numerical results from which more complex hemodynamic factors are derived. It should be recognized that ideal experimental and numerical methods are not available and it is important to be aware of the error sources they are subject to and understand how they would affect the results obtained. In PIV measurement, errors could be magnified in regions of high spatial velocity gradient and sustained low or stagnant flow. Ideally the flow within an interrogation area should be homogeneous with all seeding particles producing exactly the same displacement. In actual experiments, the displacement of individual particles may vary greatly in regions of high velocity gradient where fast moving particles are more likely than slow ones to have either the initial or the final position outside the interrogation area. This reduces substantially the signal-to-noise ratio and tends to bias the measurement towards zero. This problem has been mitigated in this study, but by no means eliminated, by increasing seeding density or offsetting correlation areas. In addition, seeding particles tend to accumulate in regions of sustained low or stagnant flow, causing light sheet reflection and locally high optical noise. This reflection will obscure the scattered light from the surrounding particles resulting in data drop out and increased errors in estimating local flow velocity. These factors could partly account for the discrepancies between the experiment and modeling specifically around the flow division point on the floor.

In the experiment, the requested flow waveform was generated by the gear pump system and measured by an electromagnetic flowmeter. Gauge calibration and testing for the pump and the flowmeter have been performed prior to the measurement to minimize possible systematic errors associated with these equipments. It has been 
shown that the experimental waveforms conform nicely to the numerical ones. However, the measured waveform may still not accurately represent the actual one entering the test section, which may increase discrepancies between the numerical and experimental data. In the flow circuit, the flowmeter is placed about $15 \mathrm{~cm}$ upstream of the test section and connected by a silicone rubber tube. The waveform may be altered as the flow passes though the silicone rubber tube. However, this error source is not considered to be significant in the present study as the silicone tube is essentially thickwalled and of limited elasticity.

Numerical results can also be inaccurate if the boundary conditions do not represent the realistic flows. The comparisons between the numerical and experimental flow fields show that the largest discrepancies occur when the flow rate is significantly lower or higher than the mean value, specifically in the outflow vessel. Take the peak flow of the LAD waveform for instance, the experimental results exhibit a smaller flow volume in the distal outflow segment but a larger volume in the proximal segment as compared to the numerical results. The discrepancies tend to be gradually reduced when the transient Reynolds number approaches the mean value. It is speculated that the discrepancies may be attributed to the flow division ratio in the proximal and distal outlet segments being altered over the flow cycle in the experimental measurement. In the present study, the proximal-distal outflow ratio was regulated by needle valves, and was set at 20:80 based on the mean flow velocity. However, this ratio could vary over the flow cycle when the transient flow velocity becomes deviated from the mean velocity. This was indeed the case as demonstrated by Loth et al. (1993). They showed that, in acute canine experiments, only $4 \%$ of the flow enters the proximal outflow segment during end diastole and $22 \%$ during peak systole when the mean proximal outflow is kept at 
about 20\%. This phenomenon has not been accurately reproduced here and could constitute the primary source of error in the predicted results.

In a numerical study simulating varying flow ratios in a femoral artery anastomosis, Lei et al. (2001) reported a maximum $\varepsilon_{r}$ of about $42 \sim 47 \%$ in WSS, about one-half that of the present study $(85.4 \%)$. Note that WSS, as a second derivative, tends to be noiser than velocity and this may contribute to another source of error. The larger relative error in the present study than in the literature suggests that assigning more accurate outflow conditions is likely to help reduce the discrepancies. Large values in relative error in the present study, however, can be a biased estimation caused by low magnitude of the experimental WSS in the denominator of the error estimation equation. In this situation, i.e. when the flow is low, it would be more appropriate to use $\varepsilon_{\text {norm }}$. For example, the largest relative error of $85.4 \%$ in the present study corresponds to the normalized error of only $1.6 \%$, i.e. about $0.74 \mathrm{dyn} / \mathrm{cm}^{2}$ absolute difference in WSS. According to the quantitative nonlinear relationship between shear stress and intimal thickness defined by $\mathrm{Ku}$ et al. (1985), $0.74 \mathrm{dyn} / \mathrm{cm}^{2}$ difference in shear results in the maximum difference of less than $0.1 \mathrm{~mm}$ in thickness of intimal ingrowth in vivo, which is not significant as compared to the size of the artery lumen (3.3 $\mathrm{mm}$ in diameter). Larger normalized errors occur where higher WSS magnitudes are delivered, and the errors are maximized at the peak flow. The largest normalized error of $21.3 \%$ in the present study corresponds to $9.8 \mathrm{dyn} / \mathrm{cm}^{2}$ absolute difference in WSS. Since intimal thickness changes slowly when WSS is high (Ku et al., 1985), a WSS difference up to $9.8 \mathrm{dyn} / \mathrm{cm}^{2}$ leads to a still minor difference in intimal thickness. It should, however, be recognized that the correspondence of WSS and intimal thickness here is by no means absolutely accurate, but it does provide a means to roughly assess the significance of discrepancies between the experiment and modeling. Despite the errors, the general 
trends of the numerical WSS curves are consistent with the experimental ones. Although using a fixed flow division ratio does affect the accuracy of the predicted results, this assumption is considered acceptable as it is not expected to yield significant difference in the severity of disease in view of the relationship between shear stress and intimal thickness.

This study was confined to idealized models which are useful in the validation protocol and studies of geometrically-controlled parameters on anastomotic hemodynamics of a generalized case. Clinical data on anastomotic geometry and corresponding IT sites, if available, would ensure better insights on interrelation between anastomotic geometries, hemodynamics and the pathogenesis of IT. Only one proximal-distal flow division ratio was investigated. It is not sure how changing the ratio will affect the discrepancies between the numerical model and PIV measurement and if the hemodynamics would be affected as has been shown in peripheral systems (Kute and Vorp et al., 2001). It will be interesting to address these issues in further studies

In conclusion, PIV was employed to validate the numerical results in a complementary approach to investigate unsteady flows in coronary anastomoses. The two approaches present excellent qualitative agreement and satisfactory quantitative agreement with averaged normalized errors in WSS between $0.8 \sim 8.9 \%$ and offer a valid numerical scheme for further studies on unsteady coronary bypass flows. 


\section{CHAPTER 8}

\section{CONCLUSIONS AND RECOMMENDATIONS}

\subsection{CONCLUSIONS}

This thesis provides insights in the interrelation between hemodynamics, anastomotic geometry and disease development in the distal coronary anastomosis. The most critical hemodynamic factors inducing disease development were determined by correlating hemodynamic parameters with histological data of disease distributions. To minimize the effects of these factors in the coronary anastomosis, subsequent geometric optimizations were performed. For reproducing and maintaining the optimized geometry in vivo, the use of a premanufactured biocompatible "sleeve" device was then proposed to connect the graft and recipient artery and its hemodynamic performance was assessed. Furthermore, PIV experimental measurements were conducted for the validation of the numerical results. Based on the above investigations, the following conclusions can be reached:

1. Intimal thickening at different sites of the coronary anastomosis is dominated by different disease-triggering mechanisms. The development of intimal thickening on the hood and graft body is likely caused by low average WSS or high OSI, and venous grafts intrinsically has a higher tendency towards thickening than arterial grafts. The thickening on the artery floor is more associated with high OSI and high SWSSG. Therefore, low WSS, high OSI and high SWSSG are the critical hemodynamic factors dominating disease development in the coronary anastomosis. 
2. The graft-artery diameter ratio is the most crucial geometric factor that surgeons should carefully control in constructing coronary anastomoses. The bypass hemodynamic performance would be potentially compromised in the anastomosis with 1:1 graft-artery diameter ratio. Extreme graft-artery diameter mismatch should also be avoided, specifically for the venous graft, so as to reduce the likelihood of rapid narrowing of the graft lumen. It is advisable for surgeons to always place an anastomosis where the graft diameter is about 1.5 times larger than the native artery diameter to achieve better hemodynamics.

3. Given the same mean Reynolds number, changing the shape of the inlet flow waveform has little impact upon the distributions of cycle-averaged WSS and SWSSG. The OSI, however, is more severe for the LAD waveform which is associated with more pulsatility and higher deceleration rate than the RCA waveform. It is suggested that bypass anastomoses in the LAD region are more prone to disease development as compared to the RCA region.

4. The effect of graft-artery angle is important, but not as significant as that of the diameter ratio. The smallest angle is associated with the highest level of OSI, whereas the largest angle results in the highest SWSSG on the artery floor. A moderate graft-artery angle between $30 \sim 45^{\circ}$ with a diameter ratio of 1.5:1 would improve hemodynamic performance of the coronary anastomosis.

5. For infragenicular bypass surgeries, suboptimal cuff performance can be caused by impaired hemodynamics as a result of inappropriate cuff configuration by inducing rapid accumulation of $\mathrm{IH}$ at the interface between the synthetic graft and the vein cuff. Specifically, it is the length-height ratio of the cuff that plays 
a critical role and should be carefully constructed. The St Mary's boot configuration is hemodynamically superior to the Miller cuff and is expected to further enhance the graft patency rates. However, the employment of these geometries in the coronary region is not recommended as it worsens aspects of hemodynamics in the critical native artery in coronary flow conditions.

6. An aspect ratio equal to or greater than 8 of the toe transition results in a significant improvement in hemodynamics as compared to the conventional geometry with a sharp toe transition. Surgery should be designed to obtain as smooth a toe transition as possible in light of fluid dynamics.

7. The sleeve technique is proposed as a solution to accurately reproduce the hemodynamically-conducive anastomotic geometry in vivo. It is suggested that the sleeve geometry gives the optimal hemodynamic performance when the proximal portion of the outflow is within $10 \sim 40 \%$ of the total graft flow. However, caution is advised in applying the sleeve to the nonplanar anastomosis since the nonplanarity tends to enlarge the low shear area on the synthetic sleeve surface leading to thrombus formation and neointimal hyperplasia. In addition, choosing an appropriate wall thickness is critical in designing the sleeve device. Specifically, the thickness of the sleeve wall should not exceed $0.08 d_{a}$.

8. There is shown to be excellent qualitative agreement between the PIV experiment and numerical simulation, and also satisfactory quantitative agreement with the largest average normalized error in WSS below $10 \%$. The discrepancies between experiment and simulation mainly result from the inadequacy of the numerical code in reproducing the cyclic variations of the proximal-distal outflow ratio. Although using a predetermined flow division 
ratio affects the accuracy of the predicted results, this assumption is considered acceptable as it would not lead to significant difference in the severity of disease in view of the relationship between shear stress and intimal thickness. The current numerical scheme is therefore deemed valid for modeling unsteady flows in coronary anastomoses.

\subsection{RECOMMENDATIONS}

Although the results of this thesis indicate causal correlations between hemodynamic factors and disease development in the distal coronary anastomosis, they are not conclusive. For the correlated histological data, intimal thickness was measured at one complete central section of the anastomotic region and five cross-sections along the graft body for all the samples. However, longitudinal sections of the anastomotic region, although more difficult to section and process, would provide more information for correlation with hemodynamic studies. In addition, the dimensions of the sample geometries were not well characterized, which made it difficult to establish accurate relationships. In the future study, correlations should be conducted based on more detailed histological data at longitudinal sections and patient-specific realistic anastomotic geometries.

Fluid dynamic boundary conditions may greatly influence the clinical relevance and applicability of the numerical results. This thesis used idealized flow conditions such as fully-developed flows at the inlet and outlet boundaries and predetermined flow division ratio in the proximal and distal artery segments, which are sometimes much different from those measured in vivo in peripheral anastomosis (Loth et al., 2002). It is important in the future numerical studies to prescribe more realistic fluid boundary conditions based on quantitative in vivo flow measurements in the coronary 
anastomosis. Numerical simulation with suitable physiologically relevant fluid boundary conditions and anastomotic geometry will exert its potentials for virtual planning of vascular surgery and design of improved surgical therapies.

In addition to hemodynamics, intramural stress concentration at graft-artery transitions as a result of compliance mismatch and presence of sutures is another critical determinant to the graft patency (Ballyk et al., 1998; Rachev et al., 2000). Further studies considering effects of suture-line stress patterns on intimal thickening in the distal coronary anastomoss are needed, which can be achieved using large strain finite element analyses of vascular wall mechanics.

This thesis proposed a new anastomotic technique with an interposition sleeve, and hemodynamic aspects of the sleeve device were examined numerically. Further biocompatibility tests in vitro and in animals (e.g. sheep or pig) are necessary. In addition, for precise placement of the sleeve device in vivo, a sleeve delivery system needs to be developed. This way, surgical procedures can be expedited and standardized, which will allow totally endoscopic or robotic executions. 


\section{REFERENCES}

Acar, C., Ramshey, A. and Pagny, J. Y., The radial artery for coronary artery bypass grafting: clinical and angiographic results at five years, J. Thorac. Cardiovasc. Surg., Vol. 116, pp. 981-989 (1998)

Adrian, R. J., Partical-imaging techniques for experimental fluid mechanics. Annu. Rev. Fluid Mech., Vol. 23, pp. 261-304 (1991)

Alevriadou, B. R., Moake, J. L., Turner, N. A., Ruggeri, Z. M., Follie, B. J., et al., Realtime analysis of shear-dependent thrombus formation and its blockade by inhibitors of von Willebrand factor binding to platelets, Blood, Vol. 81(5), pp. 1263-1276 (1993)

Allaire, E. and Clowes, A. W., The intimal hyperplastic response, Ann. Thorac. Surg., Vol. 64, pp. S38-46 (1997)

Allen, S. P., Chester, A. H., Dzimiri, N. F., Duran, C. M. G. and Yacoub, M., Vascular biology of human saphenous veins, Annals of Saudi Medicine, Vol. 5, pp. 378-384 (1995)

Thom, T., Haase, N., Rosamond, W., et al., Heart Disease and Stroke Statistics -2006 Update, Circulation, Vol. 113, pp. 85-151 (2006)

Anayiotos, A. S., Pedroso, P. D., Eleftheriou, E. C., Venugopalan, R. and Holman, W. L., Effect of a flow-streamlining implant at the distal anastomosis of a coronary artery bypass graft, Annals of Biomedical Engineering, Vol. 30, pp. 917-926 (2002)

Arnal, J. F., Schott, C., Stoclet, J. C. and Michel, J. B., Vascular relaxation and cyclic guanidine monophosphate in a rat model of high output heart failure, Cardiovasc. Res., Vol. 27, pp. 1651-1656 (1993)

Asakura, T. and Karino, T., Flow patterns and spatial distribution of atherosclerotic lesions in human coronary arteries, Circ. Res., Vol. 66, pp. 1045-1066 (1990)

Badimon L., Badimon J. J., Galvez A., Chesebro J. H. and Fuster V., Influence of arterial damage and wall shear rate on platelet deposition. Ex vivo study in a swine model, Arteriosclerosis, Vol. 6: pp. 312-20 (1986)

Ballyk, P. D., Walsh, C., Butancy, J. and Ojha, M., Compliance mismatch may promote graft-artery intimal hyperplasia by altering suture-line stresses, J. Biomech., Vol. 31, pp. 229-237 (1998)

Bao, X., Lu, C. and Frangos, J. A., Temporal gradient in shear but not steady shear stress induces PDGF-A and MCP-1 expression in endothelial cells, Arterioscler. Thromb. Vasc. Biol., Vol. 19, pp. 996-1003 (1999) 
Barner, H. B., Arterial grafting: techniques and conduits, Ann. Thorac. Surg., Vol. 66, pp. S2-S5 (1998)

Bassiouny, H. S., White, S., Glagov, S., Choi, E., Giddens, D. P. and Zarins, C. K., Anastomotic intimal hyperplasia: mechanical injury or flow induced, J. Vasc. Surg., Vol. 15, pp. 708-717 (1992)

Bates, C. J., O’Doherty, D. M. and Williams, D., Flow instabilities in a graft anastomosis: a study of the instantaneous velocity fields, Proceedings of Institution of Mechanical Engineering, Part H, Vol. 215 (6), pp. 579-587 (2001)

Berceli, S. A., Warty, V. S., Sheppeck, R. A., Mandarino, W. A., Tanksale, S. K. and Borovetz, H. S., Hemodynamics and low density lipoprotein metabolism. Rates of lowdensity lipoprotein incorporation and degradation along medial and lateral walls of the rabbit aorto-iliac bifurcation, Arteriosclerosis, Vol. 10, pp. 686-694 (1990)

Berger, K., Sauvage, L. R., Roa, A. M. and Wood, S. J., Healing of arterial prosthesis in man: its incompleteness, Ann. Surg., Vol. 175, pp. 118-127 (1972)

Bertolotti, C. and Deplano, V., Three-dimensional numerical simulations of flow through a stenosed coronary bypass, J. Biomech., Vol. 33, pp. 1011-1122 (2000)

Bertolotti, C., Deplano, V., Fuseri, J. and Dupouy, P., Numerical and experimental models of post-operative realistic flows in stenosed coronary bypasses, J. Biomech., Vol. 34, pp. 1049-1064 (2001)

Bluestein, D., Niu, L., Schoephoerster, R. T. and Dewanjee, M. K., Steady flow in an aneurysm model: correlation between fluid dynamics and blood platelet deposition, $J$. Biomech. Eng., Vol. 118(3), pp. 280-286 (1996)

Bluestein, D., Niu, L., Schoephoerster, R. T. and Dewanjee, M. K., Fluid mechanics of arterial stenosis: relationship to the development of mural thrombus, Ann. Biomed. Eng., Vol. 25, pp. 344-356 (1997)

Bourassa, M. G., Enjalbert, M., Campeau, L. and Lesperance, J., Progression of atherosclerosis in coronary arteries and bypass grafts: ten years later, Am. J. Cardiol., Vol. 53, pp. 102C-107C (1984)

Bourassa, M. G., Fisher, L. D., Campeau, L., Gillespie, M. J., McConney, M. and Lesperance, J., Long-term fate of bypass grafts: the coronary artery surgery study (CASS) and montreal heart institute experiences, Circulation, Vol. 72 (Suppl V), pp. 71-78 (1985)

Brenowitz, J. B., Kayser, K. L. and Johnson, W. D., Results of Coronary Artery Endarterectomy and Reconstruction, J. Thorac. Cardiovasc. Surg., Vol. 95, pp. 1-10 (1988)

Brumby, S. A., Petrucco, M. F., Walsh, J. A. and Bond, M. J., A retrospective analysis of infra-inguinal arterial reconstruction: three-year patency rates, Aust. NZ. J. Surg., Vol. 62, pp. 256-260 (1992) 
Buchaman Jr., J. R. and Kleinstreuer, C., Simulation of particle-hemodynamics in a partially occluded artery segment with implications to the initiation of microemboli and secondary stenosis, J. Biomech. Eng., Vol. 120, pp. 446-454 (1998)

Butany, J. W., David, T. E. and Ojha, M., Histological and morphometric analyses of early and late aortocoronary vein grafts and distal anastomoses, Can. J. Cardiol., Vol. 14, No. 6, pp. 671-677 (1998)

Cameron, A., Kemp, H. G. J. and Shimomura, S., Aortocoronary bypass surgery: a 7year follow-up, Circulation, Vol. 60, pp. 9-13 (1979)

Canfield, A. E. and Schor, A.M., Evidence that tenascin and thrombospondin-1 modulate sprouting of endothelial cells, J. Cell. Sc., Vol. 108, pp. 797-809 (1995)

Caro, C. G., Fitz-Gerald, J. M. and Schroter, R.C., Atheroma and arterial wall shear observations, correlation and proposal of a shear dependent mass transfer mechanism for atherogenesis, Proceeding of the Royal Society of London B, Vol. 177, pp. 109-159 (1971)

Clowes, A. W., Intimal hyperplasia and graft failure, Cardiovasc. Pathol., Vol. 2(suppl.), pp. 179S-186S (1993)

Chong, C. K., Rowe, C. S., Sivanesan, S., Rattray, A., Black, R. A. and Shortland, A. P., How, T.V., Computer aided design and fabrication of models for in vitro studies of vascular fluid dynamics, Proceedings of Institution of Mechanical Engineering, Part $\mathrm{H}$ Vol. 213, pp. 1-4 (1999)

Christensen, K. T. and Adrian, R. J., Measurement of instantaneous Eulerian acceleration fields by particle-image accelerometry: method and accuracy, Exp. Fluids, Vol. 33(6), pp. 759-769 (2002)

Clowes A. W., Clowes M. M. and Reidy M. A., Kinetics of cellular proliferation after arterial injury, I: smooth muscle growth in the absence of endothelium, Lab Invest., Vol. 49, pp. 327-333 (1983)

Cole, J. S., Watterson, J. K. and O’Reilly, M. J. G., Is there a haomodynamic advantage associated with cuffed arterial anatomoses? J. Biomech., Vol. 35, pp. 1337-1346 (2002a)

Cole, J. S., Watterson, J. K. and O’Reilly, M. J. G., Numerical investigation of the haemodynamics at a patched arterial bypass anastomosis, Medical engineering \& Physics, Vol. 24, pp. 393-401 (2002b)

Colman, R.W., Mechanisms of thrombus formation and dissolution. Cardiovasc. Pathol. Vol. 2(3), pp. S23-32 (1993)

Constantinides P., Cause of thrombosis in human atherosclerotic arteries, Am. J. Cardiol., Vol. 66(16), pp. G37G-40 (1990) 
Cornhill, J. F. and Roach, M. R., A quantitative study of the localization of atherosclerotic lesions in the rabbit aorta, Atherosclerosis, Vol. 23, pp. 489-501 (1976)

Crawshaw, H. I., Quist, W. C., Serallach, E., Valeri, C. R. and LoGerfo, F. W., Flow disturbance at the distal end-to-side anastomosis. effect of patency of the proximal outflow segment and angle of anastomosis, Archives of Surgery, Vol. 115, pp. 1280$1284(1980)$

Cutter, J., Tan, B. Y. and Chew, S. K., Levels of cardiovascular disease risk factors in singapore following a national intervention programme, Bulletin of the World Health Organization, Vol. 79, No. 10, pp. 908-915 (2001)

Cybulsky, M. I. and Gimbrone, M. A. Jr., Endothelial expression of a mononuclear leukocyte adhesion molecule during atherogenesis, Science, Vol. 251(4995), pp. 788791 (1991)

Da Silva A. and Carpenter T., How TV, Harris PL, Stable vortices within vein cuffs inhibit anastomotic myointimal hyperplasia? European Journal of vascular and Endovascular Suregry, Vol. 14, pp. 157-163 (1997)

Davies, P. F., Barbee, K. A., Lal, R., Robotewskyj, A. and Griem, M. L., Hemodynamics and atherogenesis: endothelial surface dynamics in flow signal transduction, Ann. NY. Acad. Sci., Vol. 748, pp. 86-103 (1995)

De Feyter, P. J., Serruys, P. W. and Brower, R.W., Comparison of preoperative, operative and postoperative variables in asymptomatic or minimally symptomatic patients to severely symptomatic patients three years after coronary artery bypass grafting: analysis of 423 patients, Am. J. Cardiol., Vol. 55, pp. 362-366 (1985)

Deng, X., Marois, Y., How, T., Merhi, Y., King, M., Guidoin, R. and Karino, T., Luminal surface concentration of lipoprotein (LDL) and its effect on the wall uptake of cholesterol by canine carotid arteries, J. Vasc. Surg., Vol. 21, pp. 135-145 (1995)

DePaola, N., Gimbrone, M. A., Jr., Davies, P. F. and Dewey, C. F., Vascular endothelium response to fluid shear stress gradients, Arterioscler. Thromb., Vol. 12(11), pp. 1254-1257 (1992)

Deters, O. J., Bargeron, C. B., Mark, F. F., et al., Measurement of wall motion and wall shear in a compliant arterial cast, ASME J. Biomech. Eng., Vol. 108, pp. 355-358 (1986)

Dickson, B. C. and Gotlieb, A. L., Endothelial dysfunction and repair in the pathegenesis of stable and unstable fibroinflammatory ahtermas, Can. J. Cardiol., Vol. 20(Suppl. B), pp. 16B-23B (2004)

Dietl, C. A., Benoit, C. H., Gilbert, C. L., Woods, E. L., Pharr W. F., Berkheimer, M. D., Madigan, N. P. and Menapace, F. J., Which is the graft of choice for the right coronary and posterior descending arteries? comparison of the right internal mammary artery and the right gastroepiploic artery, Circulation, Vol. 92, pp. II92-II97 (1995) 
Dion, R., Glineur, D., Derouck, R., Verhelst, R., Noirhomme, P., El Khoury, G., Degrave, E. and Hanet, C., Long-term clinical and angiographic follow-up of sequential internal thoracic artery grafting, Eur. J. Cardiothorac. Surg., Vol. 17, pp. 407-414 (2000)

Dobrin, P. B., Littooy, F. N., Golan, J., Blakeman, B. and Fareed, J., Mechanical and histologic changes in canine vein grafts, Journal of Surgical Research, Vol. 44(3), pp. 259-265 (1988)

Dodge, J. T., Jr, Brown, B. G., Bolson, E. L. and Dodge, H. T., Lumen diameter of normal human coronary arteries: influence of age, sex, anatomic variation, and left ventricular hypertrophy or dilation, Circulation, Vol. 86(1), pp. 232-246 (1992)

Duncan, D. D., Bargeron, C. B., Borchardt, S. E., et al., The effect of compliance on wall shear in cast of a human aortic bifurcation, ASME J. Biomech. Eng., Vol. 112, pp. 183-188 (1990)

Eagle, K. A., Guyton, R. A., Davidoff, R., Ewy, G. A., Fonger, J., Gardner, T. J., Gott, J. P., Herrmann, H. C., Marlow, R. A., Nugent, W. C., O’Connor, G. T., Orszulak, T. A., Rieselbach, R. E., Winters, W. L. and Yusuf, S., ACC/AHA Guidelines for Coronary Artery Bypass Graft Surgery: a Report of the American College of Cardiology/American Heart Association Task Force on Practice Guidelines (Committee to Revise the 1991 Guidelines for Coronary Artery Bypass Graft Surgery), J. Am. Coll. Cardiol., Vol. 34, pp. 1262-1346 (1999)

Ethier, C. R., Steinman, D. A. and Ojha, M., Comparisons between computational hemodynamics, photochromic dye flow visualization and magnetic resonance velocimetry. In Haemodynamics of Arterial Organs - Comparison of Computational Predictions With in vivo and in vitro Data (Eds. M. Collins and X. Xu), Computational Mechanics Publications, Southampton, UK (1998a)

Ethier, C. R., Steinman, D. A., Zhang, X., Karpik, S. R. and Ojha, M., Flow waveform effects on end-to-side anastomotic flow patterns, J. Biomech., Vol. 31, pp. 609-617 (1998b)

Falk, V., Diegeler, A., Walther, T., Autschbach, R. and Mohr, F., Developments in robotic cardiac surgery, Curr. Opp. Cardiol., Vol. 15, pp. 378-387 (2000)

Fei, D. Y., Thomas, J. D. and Rittgers, S. E., The effect of angle and flow rate upon hemodynamics in distal vascular graft anastomoses: a numerical model study, $J$. Biomech. Eng., Vol. 116, pp. 331-336 (1994)

Fernandez-Ortiz, A., Badimon, J. J., Falk, E., Fuster, V., Meyer, B., et al., Characterization of the relative thrombogenicity of atherosclerotic plaque components: implications for consequences of plaque rupture, J. Am. Coll. Cardiol., Vol. 23(7), pp. 1562-1569 (1994)

Ferns, G. A. A., Raines, E. W., Sprugel, K. H., Motani, A. S., Reidy, M. A. and Ross, R., Inhibition of neointimal smooth muscle accumulation after angioplasty by an antibody to PDGF, Science, Vol. 253, pp. 1129-1132 (1991) 
Fisher, R. K, How, T. V., Carpenter, T., Brennan, J. A. and Harris, P. L., Optimising miller cuff dimensions. the influence of geometry on anastomotic flow patterns, Eur $J$ Vasc Endovasc Surg., Vol. 21, pp. 251-260 (2001)

FitzGibbon, G. M., Kafka, H. P., Leach, A. J., Keon, W. J., Hooper, G. D. and Burton, J. R., Coronary bypass graft fate and patient outcome: angiographic follow-up of 5,065 grafts related to survival and reoperation in 1388 patients during 25 years, Journal of the American College of Cardiology, Vol. 28, pp. 616-626 (1996)

Freitas, C. J., Policy statement on the control of numerical accuracy, ASME J. Fluids Eng., Vol. 115, pp. 339-340 (1993)

Friedman, M. H., Deters, O. J., Mark, F. F., Bargeron, C. B. and Hutchins, G. M., Arterial geometry affects hemodynamics, Atherosclerosis, Vol. 46, pp. 225-231 (1989)

Friedman, M. H., Bargeron, C. B., Duncan, D. D., Hutchins, G. K. and Mark, F. F., Effects of arterial compliance and nonnewtonian rheology on correlations between intimal thickness and wall shear, J. Biomech. Eng., Vol. 114, pp. 317-320 (1992)

Galjee, M. A., van Rossum, A. C., Doesburg, T., Hofman, M., Falke, T. and Visser, C., Quantification of coronary artery bypass graft flow by magnetic resonance phase velocity mapping, Magnetic Resonance Imaging, Vol. 14, pp. 485-493 (1996)

Ghali, W. A., Quan, H. and Brant, R., Coronary artery bypass grafting in Canada: national and provincial mortality trends, 1992-1995, CMAJ, Vol. 159, No. 1, pp. 25-31 (1998)

Glagov, S., Giddens, D.P., Bassiouny, H., et al., Hemodynamic effects and tissue reactions at grafts to vein anastomosis for vascular access. In Vascular access for Hemodynamics-II (Eds. B.G. Sommer and M.L. Henry), pp. 3-20, Precept Press, Chicago (1991)

Gibson, C. M., Diaz, L., Kandarpa, K., Sacks, F.M., Sandor, T., Feldman, C. and Stone, P.H., Relation of vessel wall shear stress to atherosclerosis progression in human coronary arteries, Arterioscler. Thromb., Vol. 13, pp. 310-315 (1993)

Goldman, S., Copeland, J., Moritz, T., Henderson, W., Zadina, K., Ovitt, T., Doherty, J., Read, R., Chesler, E. and Sako, Y., Saphenous vein graft patency 1 year after coronary artery bypass surgery and effects of antiplatelet therapy: results of a veterans administration cooperative study, Circulation, Vol. 80, pp. 1190-1197 (1989)

Goldsmith, H. L. and Turitto, V. T., Rheological aspects of thrombosis and haemostasis: basic principles and applications, Thrombosis and Haemostasis, Vol. 55, No. 3, pp. 415-435 (1986)

Golledge, J., Vein grafts: haemodynamic forces on the endothelium-a review, Eur. J. Vasc. Endovasc. Surg., Vol. 14, pp. 333-343 (1997) 
Gosling, R. C. and King, D.H., Arterial assessment by Doppler-shift ultrasound, Proc. R. Soc. Med., Vol. 67, pp. 447-449 (1974)

Gotlieb, A. L., Langille, B. L., Wong, M. K. K. and Kim, D. W., Biology of disease: structure and function of the endothelial cytoskeleton, Lab. Invest., Vol. 65: pp. 123137 (1991)

Gould, K. L., Kirkeeide, R. L. and Buchi, M., Coronary flow reserve as a physiologic measure of stenosis severity, J. Am. Coll. Cardiol., Vol. 15, pp. 459-474 (1990)

Grondin, C. M., Lesperance, J., Bourassa, M. G. and Campeau, L., Coronary artery grafting with the saphenous vein or internal mammary artery: comparison of late results in two consecutive series of patients, Am. Thorac. Surg., Vol. 20, No. 6, pp. 605-618 (1975)

Guo, L., Steinman, D. A., Moon, B. C., Wan, W. and Millsap, R. J., Effect of distal graft anastomosis site on retrograde perfusion and flow patterns of native coronary vasculature, Ann. Thorac. Surg., Vol. 72, pp. $782-787$ (2001)

Haga, M., Yamashita, A, Paszkowiak, J., Sumpio, B. E. and Dardik, A., Oscillatory shear stress increases smooth muscle cell proliferation and Akt phosphorylation, Journal of Vascular Surgery, Vol. 37, pp. 1277-1284 (2003)

Harris, P. L. and How, T. V., Haemodynamics of cuffed arterial anastomoses, Critical Ischaemia, Vol. 9, pp. 20-26 (1999)

He, X., and Ku, D.N., Pulsatile flow in the human left coronary artery bifurcation: average conditions, ASME Journal of Biomechanical Engineering, Vol. 118, pp. 74-82 (1996)

Hellums, J. D., 1993 Whitaker lecture: biortheology in thrombosis research, Ann. Biomed. Eng., Vol. 22, pp. 445-455 (1994)

Heise, M., Krüger, U., Rückert, R., Pfitzman, R., Neuhaus, P. and Settmacher, U., Correlation of intimal hyperplasia development and shear stress distribution at the distal end-side-anastomosis, in vitro study using particle image velocimetry, Eur. J. Vasc. Endovasc. Surg., Vol. 26, pp. 357-366 (2003)

Heise, M., Schmidt, S., Krüger, U., Rückert, R., Rösler, S., Neuhaus, P. and Settmacher, U., Flow patterns and shear stress distribution of distal end-to-side anastomosis. A comparison of the instantaneous velocity fields obtained by particle image velocimetry, J. Biomech., Vol. 37, pp. 1043-1051 (2004)

Hofer, M., Rappitsch, G., Perktold, K., Trubel, W. and Schima, H., Numerical study of wall mechanics and fluid dynamics in end-to-side anastomoses and correlation to intimal hyperplasia, J. Biomech., Vol. 29, pp. 1297-1308 (1996)

Holme, P.A., Orvim, U., Hamers, M. J., Solum, N. O., Brosstad, F. R., et al., Shearinduced platelet activation and platelet microparticle formation at blood flow conditions 
as in arteries with a severe stenosis, Arterioscler. Thromb. Vasc. Biol., Vol. 17(4), pp. 646-653 (1997)

Hornik, L., Tenderich, G., Minami, K., Fassbender, D., Schulz, T. O., Beinert, B. and Koerfer, R, First experience with the St Jude Medical, Inc, Symmetry Bypass System (Aortic Connector System), The Journal of Thoracic and Cardiovascular Surgery, Vol. 125(2), pp. 414-417 (2003)

How, T. V., Rowe, C. S., Gilling-Smith, G. L. and Harris, P. L., Interposition vein cuff anastomosis alters wall shear stress distribution in the recipient artery, J. Vasc. Surg., Vol. 31, pp. 1008-1017 (2000)

Hughes, P. E. and How, T. V., Effects of geometry and flow division on flow structures in models of the distal end-to-side anastomosis, J. Biomech., Vol. 29, pp. 855-872 (1996)

Jackson, Z. S., Ishibashi, H., Gotlieb, A. I. and Langille, B.L., Effects of anastomotic angle on vascular tissue responses at end-to-side arterial grafts, J. Vasc. Surg., Vol. 34, pp. 300-307 (2001)

Jawien, A., Bowen Pope, D. F., Lindner, V., et al., Platelet-derived growth factor promotes smooth muscle migration and intimal thickening in a rat model of ballon angioplasty, J. Clin. Invest., Vol. 89, pp. 507-511 (1992)

Jian, C.Y. and Yokobori, A.T., Jr, The effect of diameter ratio between vascular substitute and blood vessel on anastomosis, Bio-medical Materials and Engineering, Vol. 9, pp. 219-231 (1999)

Jones, R. H., Kesler, K., Phillips, H. R., Mark, D. B., Smith, P. K. and Nelson, C. L., Long-term survival benefits of coronary artery bypass grafting and percutaneous transluminal angioplasty in patients with coronary artery disease, J. Thorac. Cardiovasc. Surg., Vol. 111, pp. 1013-1025 (1996)

Jou, L. D., van Tyen, R., Berger, S. A. and Saloner, D., Calculation of the magnetization distribution for fluid flow in curved vessels, Magn. Reson. Med., Vol. 35, pp. 577-584 (1996)

Kajiya, F., Ogasawara, Y., Tsujioka, K., Nakai, M., Goto, M., Wada, Y., Tadaoka, S., Matsuoka, S., Mito, K. and Fujiwara, T, Evaluation of human coronary blood flow with an 80 channel $20 \mathrm{MHz}$ pulsed Doppler velocimeter and zero-cross and Fourier transform methods during cardiac surgery, Circulation, Vol. 74(5 Pt 2):III53-60 (1986)

Keane, R. D. and Adrian, R. J., Optimization of particle image velocimeters. Part I: Double pulsed systems, Meas. Sci. Technol., Vol. 1, pp. 1202-1215 (1990)

Keynton, R. S., Rittgers, S. E. and Shu, M. C. S., The effect of angle and flow rate upon hemodynamics in distal vascular graft anastomoses: an in vitro model study, J. Biomech. Eng., Vol. 113, pp. 458-463 (1991) 
Keynton, R. S., Evancho, M. M., Sims, R. L. and Rittgers, S. E., The effect of graft caliber upon wall shear within in vivo distal vascular anastomoses, J. Biomech. Eng., Vol. 121, pp. 79-88 (1999)

Keynton, R. S., Evancho, M. M., Sims, R. L., Rodway, N. V., Gobin, A. and Rittgers, S. E., Intimal hyperplasia and wall shear in arterial bypass graft distal anastomoses: an in vivo model study, ASME Journal of Biomechanical Engineering, Vol. 123, pp. 464-473 (2001)

Kieffer, N. and Phillips, D. R., Platelet membrane glycoproteins: functions in cellular interaction, Annu. Rev. Cell Biol., Vol. 6, pp. 329-357 (1990)

King, S. B. I., Lembo, N. J. and Weintraub, W. S., A randomized trial comparing coronary angioplasty with coronary bypass surgery: Emory angioplasty versus surgery trial (EAST), N. Engl. J. Med., Vol. 331, pp. 1044-1050 (1994)

Kissin, M., Kansal, N., Pappas, P.J., DeFouw, D.O., Durán, W.N., and Hobson, R.W., Vein interposition cuffs decrease the intimal hyperplasia response of polytetrafluoroethylene bypass grafts, Journal of Vascular Surgery Vol. 31(1), pp. 69$83(2000)$

Knight, C. J., Curzen, N., Groves, P. H., Patel, D. J., Goodall, A. H. and Wright, C., Stenting for suboptimal results following balloon angioplasty significantly reduces restenosis: results of a single centre randomised trial, Circulation, Vol. 96 (Suppl 1), pp. 1-709 (1997)

Kohler, T. R., Kirkman, T. R., Kraiss, L. W., Zierler., B. K. and Clowes, A. W., Increased blood flow inhibits neointimal hyperplasia in endothelialized vascular grafts, Circ. Res., Vol. 69, pp. 1557-1565 (1991)

Kohler, T.R. and Jawien, A., Flow affects development ofintimal hyperplasia after arterial injury in rats, Circ. Res., Vol. 12, pp. 963-971 (1992)

Koyama, N., Seki, J., Harlan, J. M. and Clowes, A. W., Extracellular matrix regulates smooth muscle cell migration and contributes to the migration-inhibitory mechanism of PDGF-AA, Circulation, Vol. 90(suppl. I), pp. I-637 (1994)

Kraiss, L., Kirkman, T., Kohler, T., Zierler, B., and Clowes, A., Shear stress regulates smooth muscle proliferation and neointimal thickening in porous polytetrafluoroethylene grafts, Arterioscler. Thromb. Vol. 11, pp. 844-52 (1991)

Kraiss, L. W., Geary, R. L., Mattsson, E. J. R., Vergel, S., Au, Y. P. T. and Clowes, A. W., Acute reductions in blood flow and shear stress induce PDGF-A expression in balloon prosthetic grafts, Circ Res., Vol. 79, pp. 45-53 (1996)

Kraiss, L.W. and Clowes, A.W., Responses of the arterial wall to injury and intimal hyperplasia. In The Basic Science of Vascular Disease (Eds. A. N. Sidawy, B. E. Sumpio, and R. G. DePalma), Future Publishing Company Inc., New York, pp. 289-317 (1997) 
Ku, D. N., Giddens, D. P., Zarins, C. K. and Glagov, S., Pulsatile flow and atherosclerosis in the human carotid bifurcation - positive correlation between plaque location and oscillating shear stress, Arteriosclerosis, Vol. 5, pp. 292-302 (1985)

$\mathrm{Ku}, \mathrm{D} . \mathrm{N}$., Zhu, C., The mechanical environment of the artery. In Hemodynamic Forces and Vascular Cell Biology (Ed. B.E. Sumpio), RG Landes Company, Austin, pp. 1-23 (1993)

Kute, S. M. and Vorp, D. A., The effect of proximal artery flow on the hemodynamics at the distal anastomosis of a vascular bypass graft: computational study, J. Biomech. Eng., Vol. 123, pp. 277-283 (2001)

Langille, B. L., Blood flow-induced remodeling of the artery wall, In Flow-Dependent Regulation of vascular Function (Eds. J. A. Bevan, G. Kaley and G. Rubanyi), New York, Oxford, pp. 277 (1995)

Leask, R. L., Intimal Thickening and Hemodynamics of Human Coronary Artery Bypass Grafts, PhD. thesis, Mechanical and Industrial Engineering, University of Toronto (2002)

Lei, M., Kleinstreuer, C. and Trushey, G. A., Numerical investigation and prediction of atherogenic sites in branching arteries, ASME Journal of Biomechanical Engineering, Vol. 117, pp. 350-357 (1995)

Lei, M., Archie, J. P. and Kleinstreuer, C., Computational design of a bypass graft that minimizes wall shear stress gradients in the region of the distal anastomosis, J. Vasc. Surg., Vol. 25, pp. 637-646 (1997)

Lei, M., Giddens, D. P., Jones, S. A., Loth, F. and Bassiouny, H., Pulsatile flow in an end-to-side vascular graft model: comparison of computations with experimental data, ASME Journal of Biomechanical Engineering, Vol. 123, pp. 80-87 (2001)

Lemson, M. S., Tordoir, J. H. M., Daemen, M. J. A. P. and Kitslaar, P. J. E. H. M., Intimal Hyperplasia in Vascular Grafts, Eur. J. Vasc. Endovasc. Surg., Vol. 19, pp. 336350 (2000)

Liepsch, D., Fundamental flow studies in models of human arteries, Frontiers of Medical And Biological Engineering: The International Journal Of The Japan Society Of Medical Electronics And Biological Engineering, Vol. 5, pp. 51-55 (1993)

Lindner, V. and Reidy, M. A., Proliferation of smooth muscle cells after vascular injury is inhibited by an antibody against basic fibroblast growth factor, Proc. Natl. Acad. Sci. USA, Vol. 88, pp. 3739-3743 (1991)

Loop, F. D., Lytle, B. W., Cosgrove, D. M., Golding, L. A., Taylor, P. C. and Stewart, R.W., Free (aorta-coronary) internal mammary artery graft: late results, J. Thorac. Cardiovasc. Surg., Vol. 92, pp. 827-831 (1986) 
Loth, F., Velocity and wall shear measurements inside a vascular graft model under steady and pulsatile flow conditions, PhD. thesis, Georgia Institute of Technology, Georgia, GA, (1993)

Loth, F., Jones, S.A., Zarins, C.K., Giddens, D.P., Nassar, R.F., Glagov, S., and Bassiouny, H.S., Relative contribution of wall shear stress and injury in experimental intimal thickening at PTFE end-to-side arterial anastomoses, ASME Journal of Biomechanical Engineering, Vol. 124, pp. 44-51 (2002)

Luscher, T. J., Diederich, D., Siebenmann, R., et al., Difference between endotheliumdependent relaxation in arterial and in venous coronary bypass grafts, $N$. Engl. J. Med., Vol. 319, pp. 462-467 (1988)

Mack, M. J., Emery, R. W., Ley, L. R., Cole, P. A., Leonard, A., Edgerton, J. R., Dewey, T. M., Magee, M. J. and Flavin, T. S., Initial Experience with Proximal Anastomoses Performed with a Mechanical Connector, Ann. Thorac. Surg., Vol. 75, pp. 1866-71 (2003)

Mark, D. B., Nelson, C. L., Califf, R. M., Harrell, F. E., Lee, K. L. and Jones, R. H., Continuing evolution of therapy for coronary artery disease. initial results from the era of coronary angioplasty, Circulation, Vol. 89, pp. 2015-2025 (1994)

Markou, C. P., Hanson, S. R., Siegel and J. M., Ku, D. N., The role of high wall shear rate of thrombus formation in stenoses, Proc. ASME Biomed. Eng. Div., NYC. Adv. Bioeng., Vol. 26, pp. 555-558 (1993)

Mattsson, E. J., Kohler, T. R., Vergel, S. M. and Clowes, A. W., Increased blood flow induces regression of intimal hyperplasia, Arterioscler Thromb Vasc Biol., Vol. 17, pp. 2245-2249 (1997)

McDonald, D.A., Blood Flow in Arteries, $2^{\text {nd }}$ edition, The Willians \& Wilkins Co., Baltimore (1974)

McNeil, P. L., Muthukrishnan, L., Warder, E., et al., Growth factors are released by mechanically wounded endothelial cells, J. Cell Biol., Vol. 109, pp. 811 (1989)

Miller, J. H., Foreman, R. K., Furguson, L. and Faris, I., Interposition vein cuff for anastomosis of prosthesis to small artery, Austr. NZ. J. Surg., Vol. 54, pp. 283 (1984)

Moore, J. A., Steinman, D. A., Prakash, S., Johnston, K. W. and Ethier, C. R., A numerical study of blood flow patterns in anatomically realistic and simplified end-toside anastomoses, J. Biomech. Eng., Vol. 121, pp. 265-272 (1999)

Morris, G. E., Raptis, S., Miller, J. H. and Faris, I. B., Femorocrural grafting and regrafting: does polytetrafluoroethylene have a role? European Journal of Vascular Surgery, Vol. 7, pp. 329-334 (1993)

Myers, J.G, Moore, J.A., Ojha, M., Johnston, K.W. and Ethier, C.R., Factors influencing blood flow patterns in the human right coronary artery, Annals of Biomedical Engineering, Vol. 29, pp. 109-120 (2001) 
Munro, J. M. and Cotran, R. S., The pathegenesis of atherosclerosis: atherosclerosis and inflammation, Lab. Invest., Vol. 58(3), pp. 249-261 (1988)

Murphy, G. and Doherty, J., The matrix metalloproteinases and their inhibitors, Am. J. Respir. Cell Mol. Biol., Vol. 7, pp. 120-125 (1992)

Nakashima, Y., Plump, A. S., Raines, E. W., Berslow, J. L. and Ross, R., ApoEdeficient mice develop lesions of all phases of arteriosclerosis throughout the arterial tree, Arterioscler. Thromb. Vasc. Biol., Vol. 14, pp. 133-140 (1994)

Nazemi, M., Kleinstreuer, C., Archie, J. P. and Sorrell, F. Y., Fluid Flow and Plaque Formation in an Aortic Bifurcation, ASME Journal of Biomechanical Engineering, Vol. 111, pp. 316-324 (1989)

Nerem, R. M., Levesque, M. J. and Sato, M., Vascular dynamics and the endothelium, in Endothelium (Eds. Schmid-Schonbeine, et al.), Springer-Verlag, New York, Vol. 23, pp. 324-341 (1986)

Newby, A. C. and Zaltsman, A. B., Molecular mechanisms in intimal hyperplasia, Journal of Pathology, Vol. 190, pp. 300-309 (2000)

Nichols, W. W., Conti, C. R., Walker, W. E. and Milnor, W. R., Input impedance at the systemic circulation in man, Circ. Res., Vol. 40, pp. 451-458 (1977)

Oderich, G.S., Panneton, J.M., Yagubyan, M., Bower, T.C., Hofer, J., Noel, A.A., Sullivan, T., Kalra, M., Cherry, K.J., and Gloviczki, P., Comparison of precuffed and vein-cuffed expanded polytetrafluoroethylene grafts for infragenicular arterial reconstructions: a case-matched study, Annals of Vascular Surgery Vol. 19, pp. 49-55 (2005)

Ofili, E. O., Labovitz, A. L. and Kern, M. J., Coronary flow velocity dynamics in normal and diseased arteries, Am. J. Cardiol., Vol. 71(14), pp. 3D-9D (1993)

Ojha, M., Spatial and temporal variations of wall shear stress within an end-to-side arterial anastomosis model, J. Biomech., Vol. 26, pp. 747-753 (1994)

Ojha, M., Leask, R. L., Johnston, K. W., David, T. E. and Butany, J., Histology and morphology of 59 internal thoracic artery grafts and their distal anastomoses, Ann. Thorac. Surg., Vol. 70, pp. 1338-1344 (2000)

Okano, M. and Yoshida, Y., Influence of shear stress on endothelial cell shapes and junction complexes at flow dividers of aortic bifurcations in cholesterol-fed rabbits, Front. Med. Biol. Eng., Vol. 5(2), pp. 95-120 (1993)

Ombrellaro, M.P., Stevens, S.L., Sciarrotta, J., Freeman, M.B., Goldman, M.H., Effect of endoluminal PTFE graft placement on cell proliferation, PDGF secretion, and intimal hyperplasia, Journal of Surgical Research Vol. 63, pp. 110-114 (1996) 
Ouriel, K., Donayre, C., Shortell, C. K., Cimino, C., Donnelly, J., Oxley, D. and Green, R.M., The hemodynamics of thrombus formation in arteries, J. Vasc. Surg., Vol. 14, pp. 757-763 (1991)

Pagni, S., Storey, J., Ballen, J., Montgomery, W., Qaqish, N. K., Etoch, S. and Spence, P. A., Factors affecting internal mammary artery graft survival: how is competitive flow from a patent native coronary vessel a risk factor? J. Surg. Res., Vol. 71, pp. 172-178 (1997)

Palatianos, G. M., Bolooki, H. and Horowitz, M. D., Sequential internal mammary artery grafts for coronary artery bypass, Ann. Thorac. Surg., Vol. 56, pp. 1136-1140 (1993)

Panneton, J.M., Hollier, L.M., Hofer, J.M., and the Distaflo Trial Investigators, Multicenter randomized prospective trial comparing a pre-cuffed PTFE graft and a vein cuffed PTFE graft for infragenicular arterial bypass, Annals of Vascular Surgery Vol. 18, pp. 199-206 (2004)

Papaharilaou, Y., Doorly, D. J. and Sherwin, S. J., The influence of out-of-plane geometry on pulsatile flow within a distal end-to-side anastomosis, J. Biomech., Vol. 35, pp. 1225-1239 (2002a)

Papaharilaou, Y., Doorly, D. J., Sherwin, S. J., Peiro, J., Griffith, C., Cheshire, N., Zervas, V., Anderson, J., Sanghera, B., Watkins, N. and Caro, C. G. Combined MR imaging and numerical simulation of flow in realistic arterial bypass graft models, Biorheology, Vol. 39, pp. 525-531 (2002b)

Parisi, A. F., Folland, E. D. and Hartigan, P., A comparison of angioplasty with medical therapy in the treatment of single-vessel coronary artery disease. Veterans Affairs ACME Investigators, N. Engl. J. Med., Vol. 326, pp. 10-16 (1992)

Patel, D.J. and Vaishnav, R. N., Basic Hemodynamics and Its Role in Disease Processes, University Park Press, Baltimore, Maryland, USA (1980)

Paz, Y., Lev-Ran, O., Locker, C. and Shapira, I., Right coronary artery recascularization in patients undergoing bilateral internal thoracic artery grafting: comparison of the free internal thoracic artery with saphenous vein grafts, Interactive Cardiovascular and Thoracic Surgery, Vol. 1, pp. 93-98 (2002)

Perktold, K. and Rappitsch, G., Computer simulation of local blood flow and vessel mechanics in a compliant carotid artery bifurcation model, J. Biomech., Vol. 28, pp. 845-856 (1995)

Perktold, K., Leuprecht, A., Prosi, M., Berk, T., Czerny, M., Trubel, W. and Schima, H., Fluid dynamics, wall mechanics, and oxygen transfer in peripheral bypass anastomoses, Ann. Biomed. Eng., Vol. 30, pp. 447-460 (2002)

Pocock, S. J., Henderson, R. A., Rickards, A. F., Hampton, J. R., King, S. B. and Hamm, C. W., Meta-analysis of randomised trials comparing coronary angioplasty with bypass surgery, Lancet, Vol. 346, pp. 1184-1189 (1995) 
Prakash, S., and Ethier, C. R., Requirements for mesh resolution in 3D computational hemodynamics, ASME Journal of Biomechanical Engineering, Vol. 123, pp. 134-144 (2001)

Prasad, A. K., Particle imaging velocimetry, Current Science, Vol. 79(1), pp. 51-60 (2000)

Privitera, S., Coronary artery bypass surgery: historical overview and future directions, University of Toronto Medical Journal, Vol. 79, pp. 12-15 (2001)

Raffel, M., Willert, C., Kompenhans, J., Particle image velocimetry, a particle guide, Springer-Verlag, New York (1998)

Reardon, M. J., Conklin, L. D., Reardon, P. R. and Baldwin, J. C. Coronary artery bypass conduits: review of current status, J. Cardiovasc. Surg., Vol. 38(3), pp. 201-209 (1997)

Rieder, M. J., Carmona, R., Krieger, J. E., Pritchard, K. A. Jr. and Greene, A. S., Suppression of angiotension-converting enzyme expression and activity by shear stress, Circ. Res., Vol. 80, pp. 312-319 (1997)

Roche, J. K. and Stengle, J. M., Open heart surgery and the demand for blood, J. Am. Med. Assoc., Vol. 225, pp. 1516-1521 (1973)

Rosenfeld, M. E., Tsukada, T., Gown, A. M. and Ross, R., Fatty streak initiation in Watanabe Heritable Hyperlipemic and comparably hypercholesterolemic fat-fed rabbits, Arteriosclerosis, Vol. 7, pp. 9-23 (1987)

Ross, R., Atherosclerosis: current understanding of mechanisms and future strategies in therapy, Transplantation Proceedings, Vol. 25(2), pp. 2041-2043 (1993)

Ross, R., Cell biology of atherosclerosis, Annu. Rev. Physiol., Vol. 57, pp. 791-804 (1995)

Rutherford, R.B., Vascular Surgery, pp. 404-486, Philadelphia, Saunders (1989)

Sabiston, D. C., The coronary circulation, Johns Hopkins Med. J., Vol. 314 (1974)

Salam, T.A., Lumsden, A.B., Suggs, W.D. and Ku, D.N., Low shear stress promotes intimal hyperplasia in a dose-response manner, J. Vasc. Investig. Vol. 2(1), pp. 12-22 (1996)

Satcher, R. L. and Dewey, C. F., The distribution of fluid forces on arterial endothelial cells, Adv. Bioeng., Vol. BED-20, pp. 595-598 (1991)

Savage, B., Saldivar, E. and Ruggeri, Z. M., Initiation of platelet adhesion by arrest onto fibrinogen or translocation on von Willebrand factor, Cell, Vol. 84(2), pp. 289-297 (1996) 
Schoephoerster, R. T., Oynes, F., Nunez, G., Kapadvanjwala, M. and Dewanjee, M. K., Effects of local geometry and fluid dynamics on regional platelet deposition on artificial surfaces, Arterioscler. Thromb., Vol. 13(12), pp.1806-1813 (1993)

Shelton, M. E., Forman, M. B., Virmani, R., Bajaj, A., Stoney, W. S. and Atkinson, J. B., A comparison of morphologic and angiographic findings in long-term internal mammary artery and saphenous vein bypass grafts, J. Am. Coll. Cardiol., Vol. 11, pp. 297-307 (1988)

Shennib, H., A renaissance in cardiovascular surgery: endovascular and device-based revascularization, Ann. Thorac. Surg., Vol. 72, pp. S993-994 (2001)

Sherwin, S. J., Shah, O., Doorly, D. J., Peiró, J., Papaharilaou, Y., Watkins, N., Caro, C. G. and Dumoulin, C. L., The influence of out-of-plane geometry on the flow within a distal end-to-side anastomosis, ASME Journal of Biomechanical Engineering, Vol. 122, pp. 86-95 (2000)

Singh, R. M. and Sosa, J. A., Internal mammary artery: a "live” conduit for coronary bypass, J. Thorac. Cardiovasc. Surg., Vol. 87, pp. 936-943 (1984)

Sisto, T. and Isola, J., Incidence of atherosclerosis in the internal mammary artery, Ann. Thorac. Surg., Vol. 47(6), pp. 884-886 (1989)

Song, M., Sato, M. and Ueda, Y., Three-dimensional simulation of Coronary Artery Bypass Grafting with the Use of Computational Fluid Dynamics, Surgery Today, Vol. 30, pp. 993-998 (2000)

Stangeby, D. K. and Ethier, C. R., Computational analysis of coupled blood-wall arterial LDL transport, J. Biomech. Eng., Vol. 124, pp. 1-7 (2002)

Steinberg, D., Lipoproteins and the pathogenesis of atherosclerosis, Circulation, Vol. 76(3), pp. 508-514 (1987)

Steinman, D. A. and Either, C. R., Numerical modeling of flow in a distensible end-toside anastomosis, Bioengineering Conference, ASME BED-Vol. 24, pp. 379-382 (1993)

Steinman, D., Vinh. B., Either, C., Ojha, M., Cobbold, R. and Johnson, K., A numerical simulation of flow in a two-dimensional end-to-side anastomosis model, ASME $J$. Biomech. Eng., Vol. 115, pp. 112-118 (1993)

Sterpetti, A. V., Schultz, R. D. and Bailey. R. I., Endothelial cell seeding after carotid endarterectomy in a canine model reduces platelet uptake, Eur. J. Vasc. Surg., Vol. 6, pp. 390-394 (1992)

Sterpetti, A. V., Cucina, A., Lepidi, S., et al., Progression and regression of myointimal hyperplasia in experimental vein grafts depends on platelet-derived growth factor and basic fibroblastic growth factor production, J. Vasc. Surg., Vol. 23, pp. 568-575 (1996) 
Stonebridge, P. A., Howlett, R., Prescott, R. and Ruchley, C. V., Randomized trial comparing polytetrafluoroethylene graft patency with and without a Miller cuff, $\mathrm{Br} . J$. Surg., Vol. 82, pp. 555-556 (1995)

Taylor, R. S., Loh, A., McFarland, R. J., Cox, M. and Chester, J. F., Improved technique for polytetrafluoroethylene bypass grafting: long-term results using anastomotic vein patches, British Journal of Surgery, Vol. 79, pp. 348-354 (1992)

Tozzi, P., Corno, A. F. and Segesser, L. K., Sutureless coronary anastomoses: revival of old concept, Eur. J. Cardio-thoracic. Surg., Vol. 22, pp. 565-570 (2002)

Trehan, N., Mishra, M., Sharma, O. P., Mishra, A. and Kasliwal, R. R., Further reduction in stroke after off-pump coronary artery bypass grafting: a 10-year experience, Ann. Thorac. Surg., Vol. 72, pp. S1026-1032 (2001)

Trubel, W., Schima, H., Czerny, M., Perktold, K., Schimek, M.G., and Polterauer, P., Experimental comparison of four methds of end-to-side anastomosis with expanded polytetrafluoroethylene, British Journal of Surgery Vol. 91, pp. 159-167 (2004)

Truskey, G. A., Barber, K. M., Robey, T. C., Olovier, L. A. and Combs, M. P., Characterization of a sudden expansion flow chamber to study the response of endothelium to flow recirculation, J. Biomech. Eng., Vol. 117, pp. 203-210 (1995)

Tyrrell, M. R. and Wolfe, J., New prosthetic venous collar anastomotic technique: combining the best of other procedures, Br. J. Surg., Vol. 78, pp. 1016-1017 (1991)

Verma, S. and Anderson, T. J., Fundamentals of emdothelial function for the clinical cardiologist, Circulation, Vol. 105, pp. 546-549 (2002)

Vyalov, S., Langille, B. L. and Gotlieb, A. I., Decreased blood flow rate disrupts endothelial repair in vivo, Am. J. Pathol., Vol. 149, pp. 2107-2118 (1996)

Wada, S. and Karino, T., Theoretical prediction of low-density lipoproteins concentration at the luminal surface of an artery with a multiple bend, Ann. Biomed. Eng., Vol. 30, pp. 778-791 (2002)

Walpola, P. L., Gotlieb, A. I. and Langille, B.L., Monocyte adhesion and changes in endothelial cell number, morphology, and F-actin distribution elicited by low shear stress in vivo, Am. J. Pathol., Vol. 142, pp. 1392-1400 (1993)

Walpola, P. L., Gotlieb, A. I., Cybulsky, M. I. and Langille, B. L., Expression of ICAM-1 and VCAM-1 and monocyte adherence in arteries exposed to altered shear stress, Arterioscler. Thromb. Vasc. Biol., Vol. 15, pp. 2-10 (1995)

Walpoth, B. H., Müller, M. F., Genyk, I., Aeschbacher, B., Kipfer, B., Althaus, U. and Carrel, T. P., Evaluation of coronary bypass flow with color-Doppler and magnetic resonance imaging techniques: comparison with intraoperative flow measurements, Eur. J. Cardiothorac. Surg., Vol. 15, pp. 795-802 (1999) 
Wang, D. M. and Tarbell, J. M., Nonlinear analysis of oscillatory flow, with a nonzero mean, in an elastic tube (artery), J. Biomech. Eng., Vol. 117, pp. 127-135 (1995)

Weston, M. W., Rhee, K. and Tarbell, J. M., Compliance and diameter mismatch affect the wall shear rate distribution near an end-to-end anastomosis, J. Biomech., Vol. 29, pp. 187-198 (1996)

White, S. S., Zarins, C. K., Giddens, D. P., Bassiouny, H., Loth, F., Jones, S. A. and Glagov, S., Hemodynamic patterns in two models of end-to-side vascular graft anastomoses: effects of pulsatility, flow division, reynolds number, and hood length, ASME Journal of Biomechanical Engineering, Vol. 115, pp. 104-110 (1993)

Wijesinghe, L. D., Smye, S. W., Scott, J. A., Impedance index measurements of in vitro PTFE end-to-side anastomoses: effect of angle and Miller cuff, Eur. J. Vasc. Surg., Vol. 82, pp. 555-556 (1998)

Wijesinghe, L. D., Mahmood, T., Scott, J. A., Axial flow fields in cuffed end-to-side anastomoses: effect of angle and disease progression, European journal of Vascular and Endovascular Surgery, Vol. 18, pp. 240-244 (1999)

Yacoub, M. H. and Radley-Smith, R., Anatomy of the coronary arteries in transposition of the great arteries and methods for their transfer in anatomical correction, Thorax, Vol. 33, pp. 418-424 (1978).

Zamir, The Physics of Coronary Blood Flow, Springer Science+Business Media, Inc., New York (2005)

Zarins, C. K., Giddens, D. P., Bharadvaj, B. K., Sottiurai, V. S., Mabon, R. F. and Glagov, S., Carotid bifurcation atherosclerosis: quantitative correlation of plaque location with flow velocity profiles and wall shear stress, Circ. Res., Vol. 53, pp. 502514 (1983)

Ziegler, T., Bouzourene, K., Harrison, V. J., Brunner, H. R., Hayoz, D., Influence of oscillatory and unidirectional flow environments on the expression of endothelin and nitric oxide synthase in cultured endothelial cells, Arterioscler. Thromb. Vasc. Biol., Vol. 18, pp. 686-692 (1998)

Zwolak, R. M., Kirkman, T. R. and Clowes, A. W., Atherosclerosis in rabbit vein grafts. Arteriosclerosis, Vol. 9, pp. 374-479 (1989) 


\section{APPENDICES}

\section{A FLOW RESULTS FOR MILLER CUFF MODELS IN INFRAGENICULAR FLOW CONDITIONS}

\section{A.1 Flow Conditions}

The input flow waveform (Fig. A.1) and physiological parameters were in reference to the experimental study of Fisher et al. (2001), with a mean flow rate of $130 \mathrm{ml} / \mathrm{min}$ and a pulse rate of 60 beats/min. Different flow division ratios in the proximal and distal outlet segments (i.e. 50:50 and 20:80) were specified.

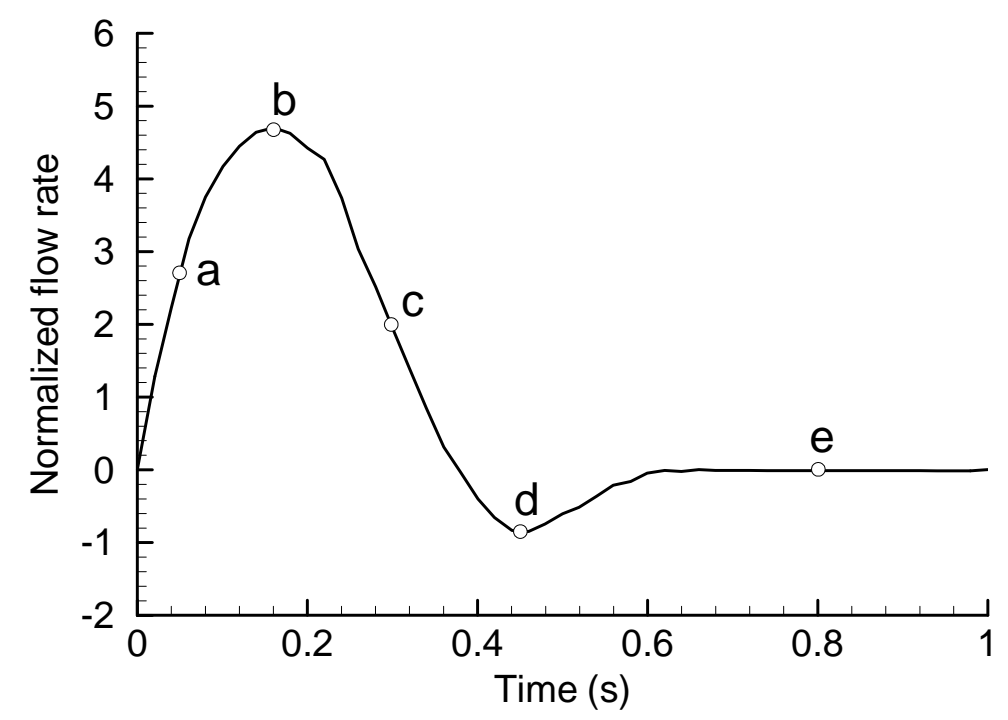

Fig. A.1 Plot of the flow waveform used for the study. The flow rate is normalized with respect to the mean flow rate, $130 \mathrm{ml} / \mathrm{min}$. Labels indicate time steps at which flow patterns are presented.

\section{A.2 Results: 50:50 Flow Division Ratio}

Flow patterns at five phases of the flow cycle shown in Fig. A.1, i.e. (a) the acceleration,

(b) peak flow, (c) mid-deceleration, (d) early diastole, and (e) late diastole, are displayed in Fig. A.2 to A.6 for all the models investigated. Detailed flow 
characteristics are described for the high cuff model (Fig. A.2). For each of the other models, only flow features different from those of the high cuff are presented.

\section{A.2.1 High Miller Cuff}

During the acceleration phase, the flow within the cuff is laminar and less disturbed The flow division point on the host artery floor resides exactly at the middle position (X $=0$ ) between the proximal and distal walls of the cuff. The flow profiles at the entrances of the proximal and distal outlet segments are skewed towards the floor, resulting in small areas of low flow on the artery ceiling just next to the cuff toe and heel.

At the peak flow, distinct flow separation arises in three locations around the graft toe, on the ceiling of the distal artery, and on the proximal cuff wall. The flow skewing in the distal artery segment becomes more severe, and there exists reverse flow immediately distal to the cuff toe, extending about $1.0 d_{a}$ longitudinally. A vortex-like structure is developed in the proximal half of the cuff, which moves the flow division point on the floor distally to $\mathrm{X}=0.8 d_{a}$.

A cohesive, clockwise-rotating vortex is gradually developed as the flow continues to decelerate from the peak flow. At mid-deceleration, this vortex occupies the majority of the cuff cavity and the area of flow separation and recirculation at the graft toe is also accordingly reduced. The flow reversal and separation distal to the cuff toe remains prominent. The flow division point on the floor drifts further distally to $\mathrm{X}=1.4 d_{a}$. The robust nature of the vortex is preserved throughout the remaining period of the deceleration. 
The flow in the entire model becomes retrograde during the early period of the negative systolic phase, which shifts the core of the vortex to the right-top quarter of the cuff. Flow separation around the graft toe and cuff toe is completely eliminated. There is a small low flow area present along the lower part of the distal cuff wall. An extent of about $2.0 d_{a}$ in the migration of the division point along the floor with respect to the position during deceleration is observed.

The flow is retrograde and generally low during the rest of the flow cycle. The vortex remains stable and cohesive, with a circulating velocity higher than that of the fluid in the graft and outflow artery segments as measured by the lengths of the velocity vectors. The stagnation point on the floor is less dynamic than during the diastolic phase. These flow features persists till the end of the cycle.

In summary, the high cuff generates a cohesive vortex which is sustained for more than three-quarters of the flow cycle (i.e. from the beginning of deceleration to the end of the cycle). The flow separation at the graft toe is most prominent at peak flow and gradually disappears as the cycle proceeds, lasting for approximately $0.15 \mathrm{~s}$. The flow reversal and separation distal to the cuff toe is also a transient phenomenon with duration of $0.2 \mathrm{~s}$. The maximum extension the flow stagnation point travels along the floor is $2.0 d_{a}$, i.e. 0.57 relative to the cuff length.

\section{A.2.1 Low Miller Cuff}

The gross flow patterns occurring in the low cuff model resemble those in the high cuff model (Fig. A.3). The most prominent differences reside in the main vortex structure and the flow recirculation at the graft toe. The vortex during deceleration, which is shown to be most robust in the high cuff model, develops into a complex structure 
without a clear core of rotation in the low cuff model. The vortex disappears during early systole and is replaced by an extensive area of flow stasis along the entire distal cuff wall. Flow recirculation and separation occupies a substantially larger area around the graft toe than is seen in the high cuff. This area grows even more prominent from the peak flow to mid-deceleration, resulting in a longer duration (about $0.2 \mathrm{~s}$ ) than in the high cuff case. During the early systole, although no clear flow separation point can be detected at the graft toe, the flow in the vicinity is almost stagnant. The maximum displacement of the stagnation point along the floor is $1.6 d_{a}$, i.e. 0.46 relative to the cuff length.

\section{A.2.1 Long Miller Cuff}

Seriously disturbed flow patterns are observed in the long cuff, which are very different from those in the high and low cuffs (Fig. A.4). Note that the long cuff seems to detain the generation of the vortex. During the deceleration phase, the majority of the cuff cavity is filled with disorganized flow structures with highly irregular fluid behavior, which are sustained till the end of the deceleration. Meanwhile, a remarkably extensive area of flow recirculation and separation is formed around the graft toe and sustained from the peak flow to the early systole $(>0.3 \mathrm{~s})$. This area is replaced by a nearly stagnant flow during the rest of the cycle. In addition, the long cuff causes the stagnation point on the floor to travel for a long distance of $2.8 d_{a}$, i.e. 0.54 relative to the cuff length.

\section{A.2.4 St Mary's Boot}

The main vortical flow in the boot is identical to that in the high cuff in terms of duration and robustness (Fig. A.5). The vortex begins to develop at the peak flow and grows increasingly expanded and cohesive as the deceleration continues. The most 
distinct difference in the boot from the cuff group is that flow separation and recirculation at the graft toe is almost completely removed with the removal of the cuff "shoulder". Flow separation distal to the cuff toe, though still present during the deceleration, is greatly alleviated. The displacement of the stagnation point $\left(3.0 d_{a}\right)$ is larger than in any of the cuff models.

\section{A.3 Results: 20:80 Flow Division Ratio}

For the 20:80 proximal-distal flow division ratio, only flow patterns in the deceleration phase are presented as shown in Fig. A.6. Generally, the influence of altering the flow division ratio on the gross flow structures is similar for each model. Increasing the distal outflow portion tends to promote and stabilize the main vortical flow within the cuff/boot, though the vortex in the low and long cuffs remains an incomplete structure. The flow separation at the graft toe in all the cuff models is also apparently subdued. The extent of flow reversal and separation along the artery ceiling distal to the cuff/boot toe seems unchanged in contrast to the case of 50:50 flow division ratio. An overall smaller displacement than in the 50:50 flow division ratio is detected for each model. 

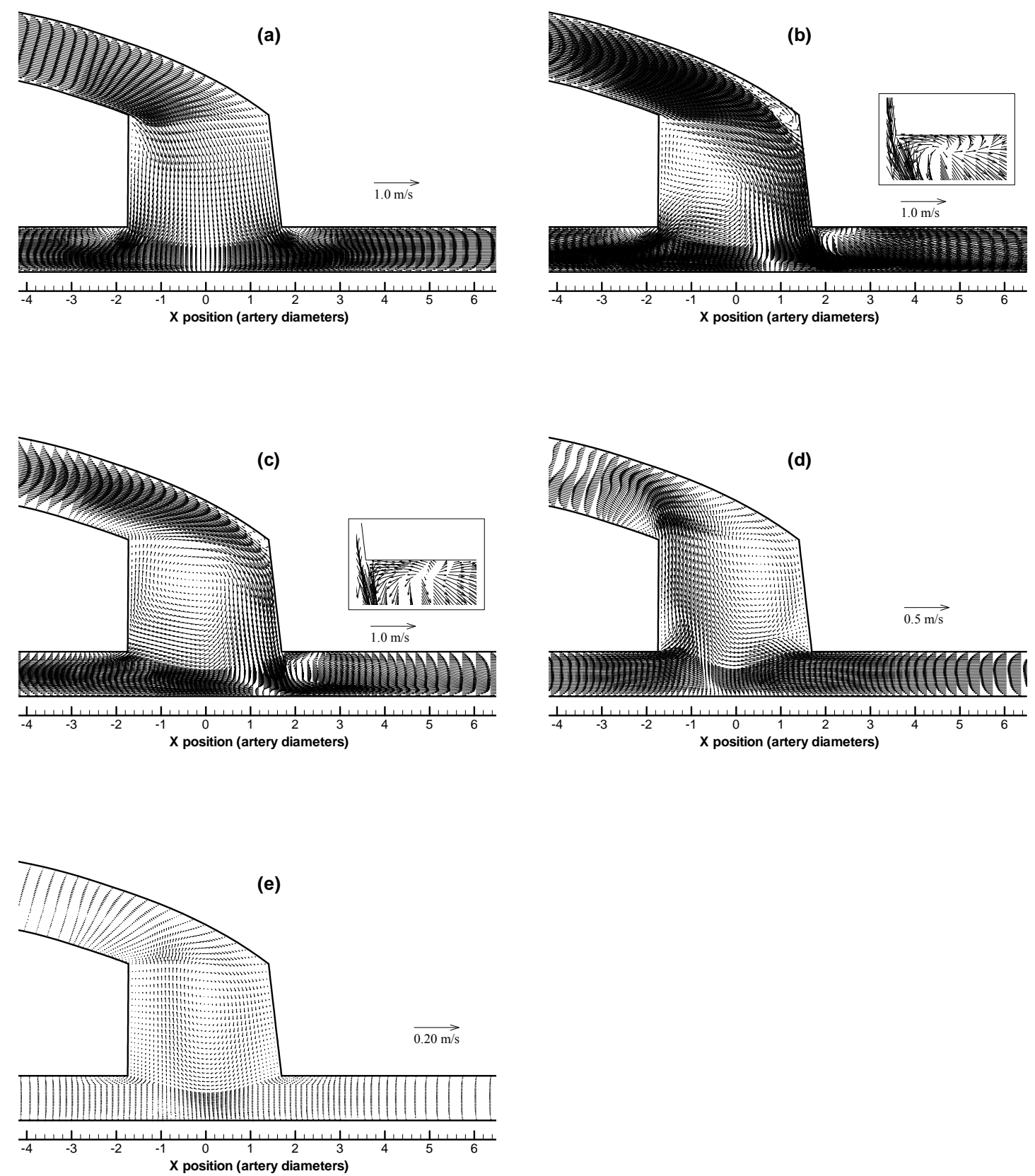

Fig. A.2 Velocity vector plots in the symmetry plane for the high Miller cuff model during (a) acceleration, (b) peak flow, (c) mid-deceleration, (d) early diastole, and (e) late diastole. 
(a)

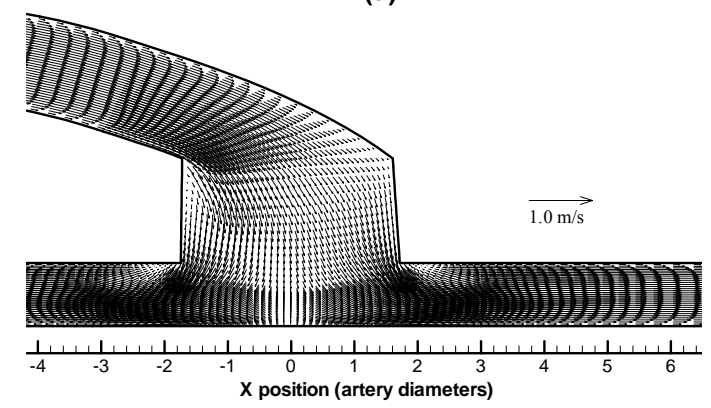

(c)

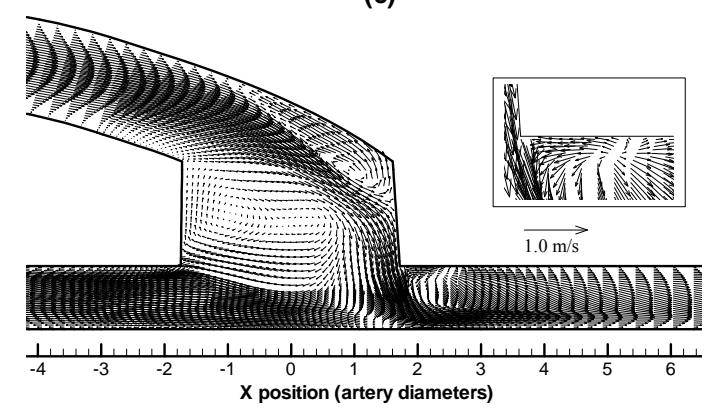

(e)

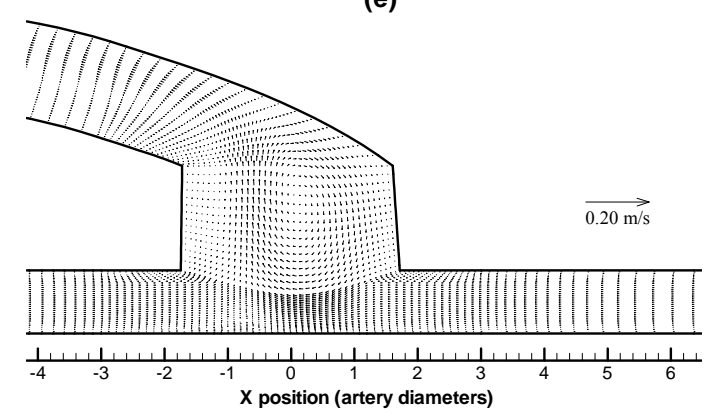

(b)

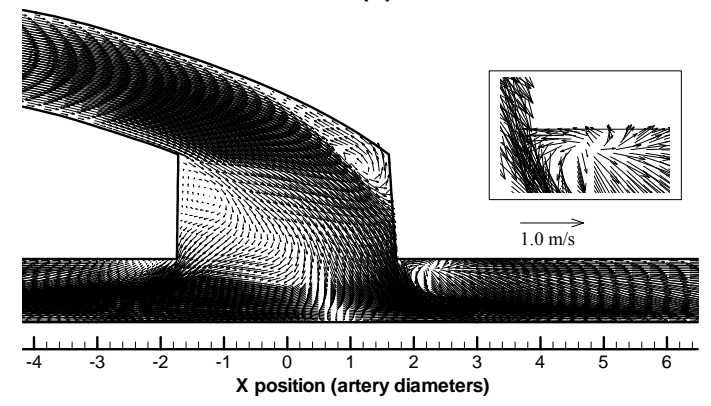

(d)

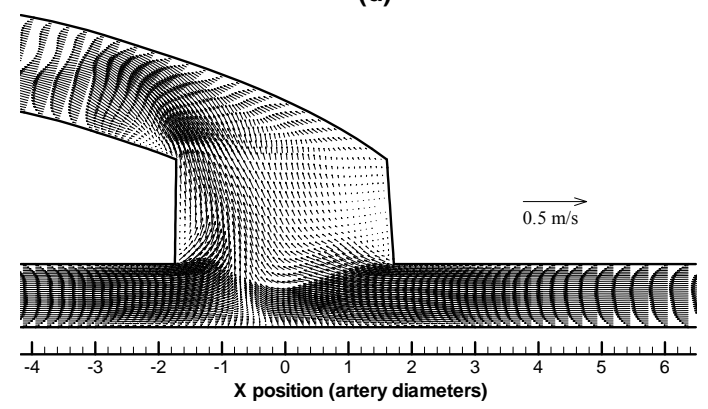

Fig. A.3 Velocity vector plots in the symmetry plane for the low Miller cuff model during (a) acceleration, (b) peak flow, (c) mid-deceleration, (d) early diastole, and (e) late diastole. 
(a)

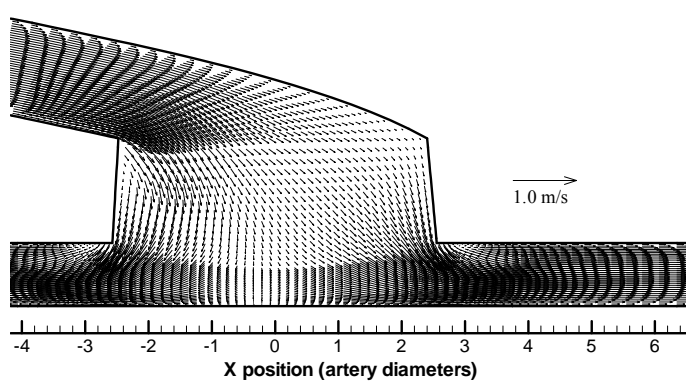

(c)

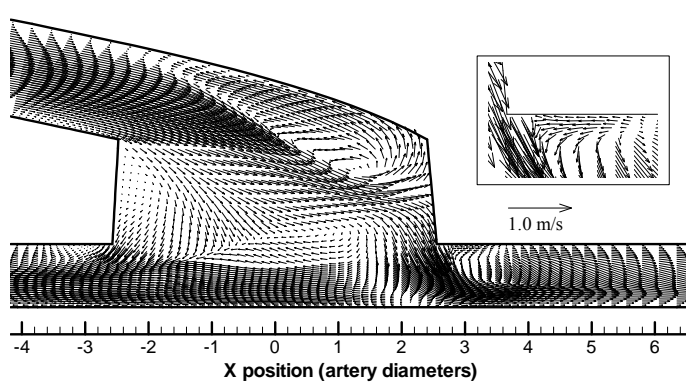

(e)

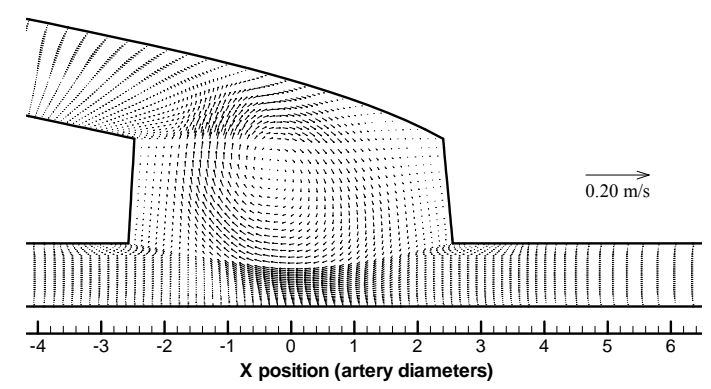

(b)

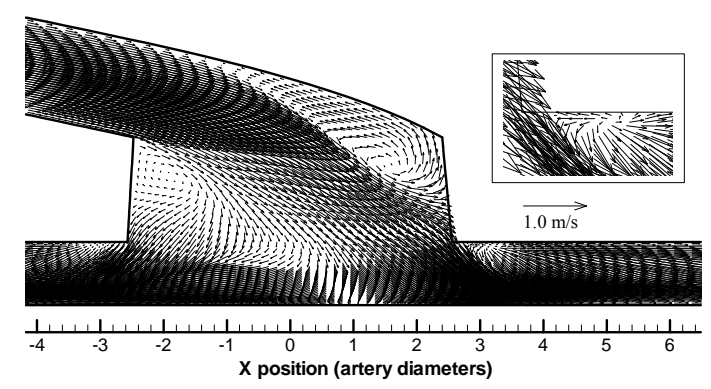

(d)

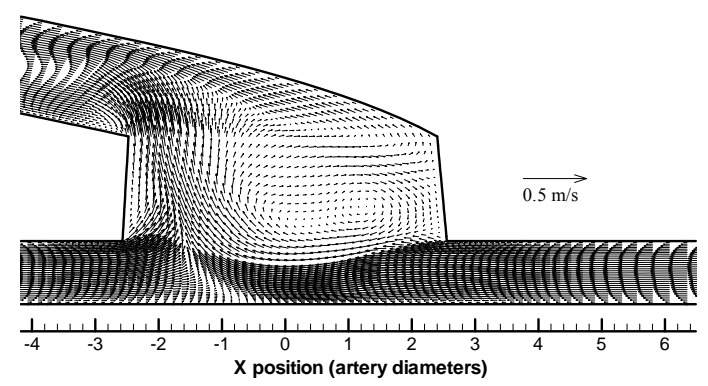

Fig. A.4 Velocity vector plots in the symmetry plane for the long Miller cuff model during (a) acceleration, (b) peak flow, (c) mid-deceleration, (d) early diastole, and (e) late diastole. 



Fig. A.5 Velocity vector plots in the symmetry plane for the St Mary's boot model during (a) acceleration, (b) peak flow, (c) mid-deceleration, (d) early diastole, and (e) late diastole. 


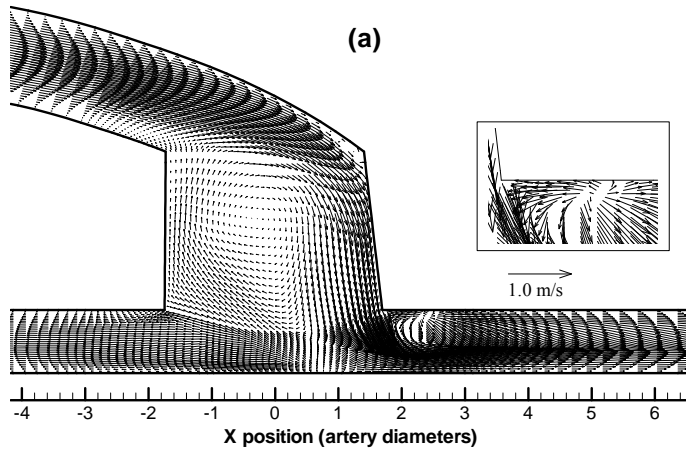

(b)

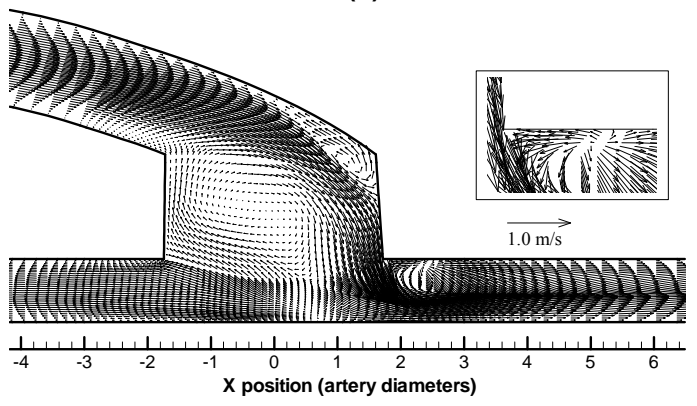

(c)
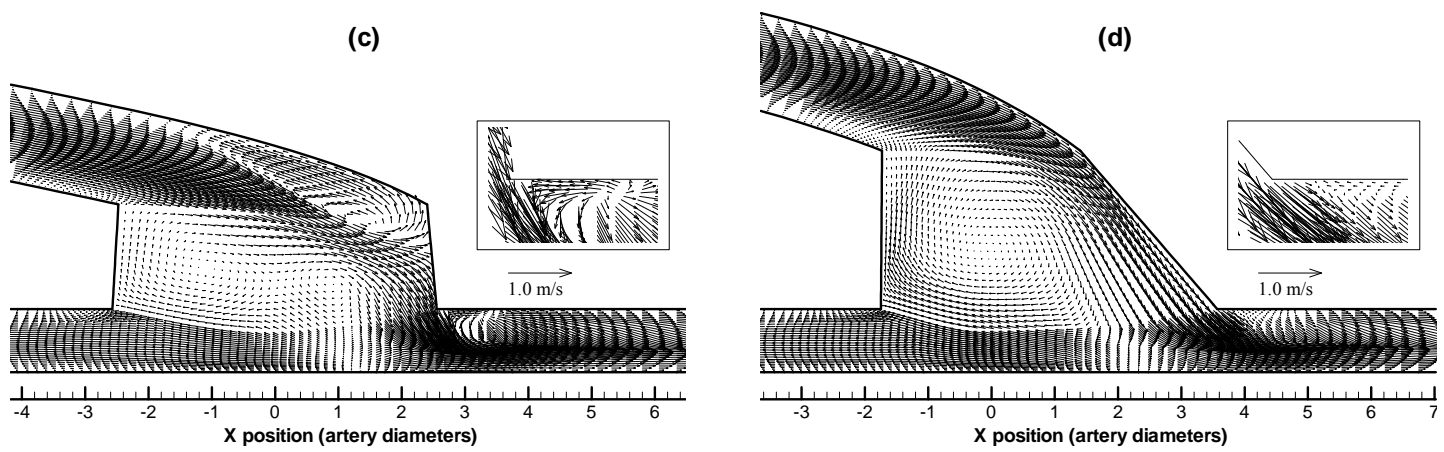

Fig. A.6 Velocity vector plots at the mid-deceleration in the symmetry plane with a proximal-distal outflow division ratio of 20:80: (a) high cuff, (b) low cuff, (c) long cuff, and (d) St Mary's boot.

\section{B EXPERIMENTAL APPARATUS}

\section{B.1 Gear Pump System}

The gear pump system consists of the AC and DC pumps (Fig. B.1a), gear pump interface unit, data acquisition board and calming chamber (Fig. B.1b). The AC pump speed is modulated by means of an AC voltage signal and generates the oscillatory component of a waveform with completely forward flow. The DC pump is controlled by means of a precision 10-turn potentiometer and operates at a constant speed to generate a steady flow component. To produce bi-directional pulsatile flow, the output from the DC pump was subtracted from that from the AC pump. The gear pump 
interface unit provides the power and control voltages for the AC and DC pumps, and is linked to the computer by the $2 \times 50$-way ribbon cables.

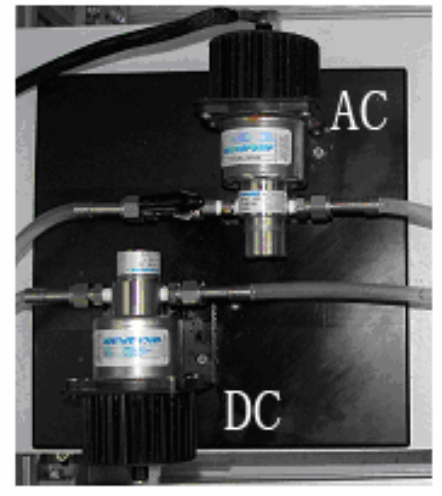

(a)

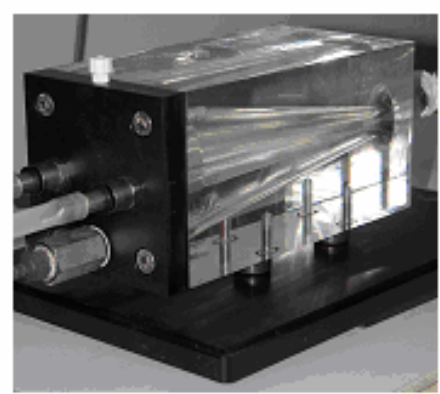

(b)

Fig. B.1 Pictures of (a) the gear pumps and (b) the calming chamber.

\section{B. 2 Illumination System}

The picture of the illumination system is shown in Fig. B.2. The core of the system is a dual-head, pulsed Gemini laser, which is designed to provide a highly stable, pulsed green light source for PIV applications. Figure B.3 shows the layout of the laser head unit, and the specifications of the laser are listed in Tab. A.1. Two Nd:YAG infrared laser heads are mounted on a single baseplate. The $400 \mathrm{~mJ}$ of input infrared beams at $1064 \mathrm{~nm}$ are polarization combined and then enter a second harmonic generator (SHG) to produce $200 \mathrm{~mJ}$ of output visible light at $532 \mathrm{~nm}$. The output power is stable to $\pm 5 \%$ for $95 \%$ of the shots. Dichroic mirrors separate the visible from the residual infrared light and direct the beam to the experiment. The laser heads, each operating at $15 \mathrm{~Hz}$, can be fired independently of each other with a specified time delay between pulses. In PIV, two successive images are captured, and the particles in one image correlated with the particles in the next. The dual-head design makes it possible to compare subsequent images with sufficiently short time delays to track particle motion. 
APPENDICES

The power supply controls the Gemini laser, supplying the required power and signals to safely operate the laser. In the closed loop water cooling system, which is located on the lower level of the power supply, a water pump recirculates the deionized water from the water-to-air heat exchanger to the laser head. The water circulates through the pump chamber to keep the temperature of the YAG rod at an acceptable level. The cooling control board controls the fan attached to the heat exchanger and monitors the temperature sensors to ensure that the laser operates reliably.

Communications between the pulsed laser and the synchronization unit are required for controlling the data acquisition process. The synchronization unit instructs the laser heads when to fire their flash lamps and when to allow their Q-switches to emit laser radiation.

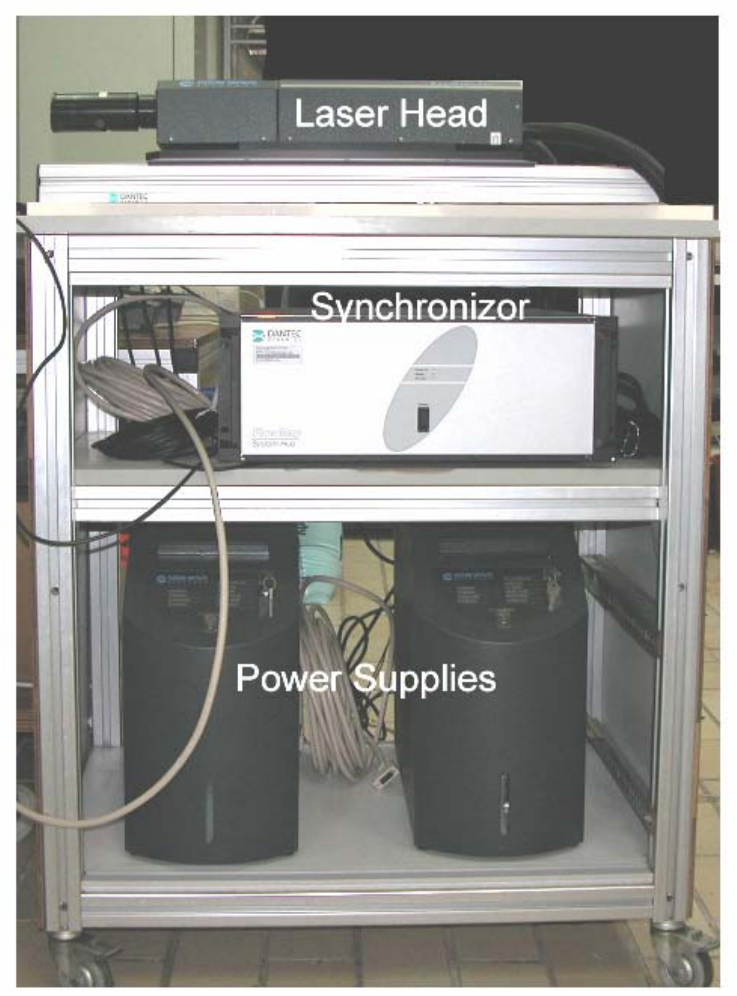

Fig. B.2 Picture of the illumination system. 


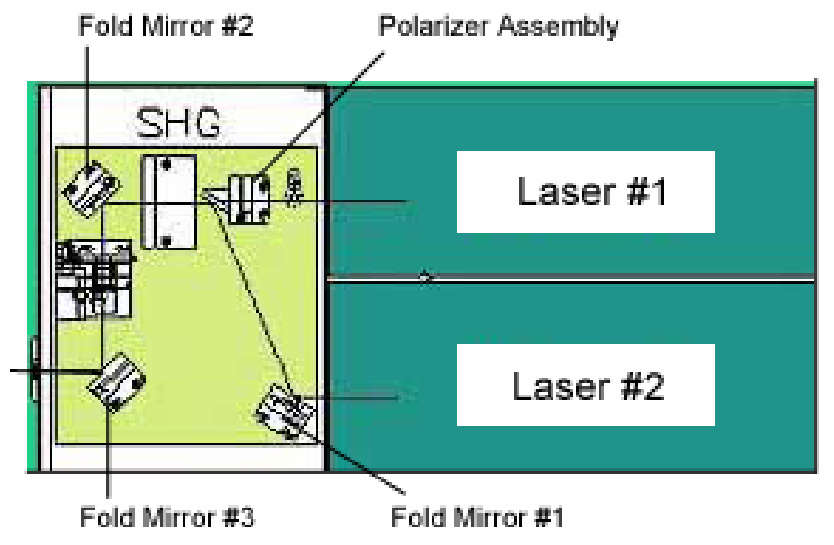

Fig. B.3 Layout of the Gemini laser head.

Tab. B.1 Gemini PIV Performance Specifications.

\begin{tabular}{ll}
\hline Energy @ 532 nm per head & $200 \mathrm{~mJ} @ 15 \mathrm{HZ}$ \\
\hline Beam Diameter & $\sim 5.5 \mathrm{~mm}$ \\
\hline Energy Stability @ 532 & $\pm 5 \%$ \\
\hline Pulse Width & $3-5 \mathrm{~ns}$ \\
\hline Beam Divergence & $<2 \mathrm{mrad}$ \\
\hline Beam Pointing & $<200 \mu \mathrm{rad}$ \\
\hline Jitter & $\pm 0.5 \mathrm{~ns}$ \\
\hline
\end{tabular}

\section{B. 3 CCD Camera}

An 80C77 HiSense MkII CCD camera with a Nikon Micro-Nikkor lens 60/f2.8 was used for the PIV study. The picture and specifications of the camera are displayed in Fig. B.4 and Tab. B.2, respectively. The camera contains a high-performance progressive scan interline CCD chip, which has $1344 \times 1024$ light sensitive pixels and storage pixels. The progressive scan interline transfer CCD is attractive for high speed flow measurement due to its short time requirement for charge transfer. The camera is driven in the asynchronous reset mode. The asynchronous resetting allows exposure of 
video frames to be synchronized to a trigger signal from the experiment. During an image acquisition sequence, the first laser pulse is fired to expose the first frame, which is stored with extreme rapidity into a buffer pixel located immediately adjacent to every sensor pixel. After a specified time delay, the second laser pulse is fired to expose the second frame. The two frames are transferred sequentially to the computer memory in time via the high-speed camera interface for the next double-frame capture and crosscorrelation of the images.

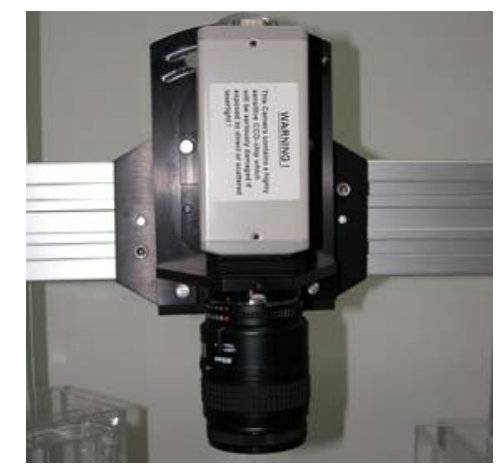

Fig. B.4 Picture of the HiSense MkII CCD camera.

Tab. B.2 Specifications of the CCD camera.

\begin{tabular}{ll}
\hline Dimensions $(\mathrm{L} \times \mathrm{W} \times \mathrm{H})$ & Camera Housing $135 \times 65 \times 65 \mathrm{~mm}$ \\
\hline Lens mount & C-mount \\
\hline CCD type & Progressive scan interline, Sony ER150 \\
\hline Active pixels & $1344 \times 1024$ pixel \\
\hline Camera bit resolution & 12 -bit \\
\hline Pixel pitch & $6.45 \times 6.45 \mu \mathrm{m}$ \\
\hline Dynamic range & $1800: 1$ \\
\hline Readout noise & 10 electrons r.m.s \\
\hline Pixel clock rate & $14.7 \mathrm{MHz}$ \\
\hline Microlens array & Standard \\
\hline Max. single-frame rate & 12.2 \\
\hline Pixel binning & Yes \\
\hline Pulse interval range & $0.3 \mu$ s and up \\
\hline Double-frame rate & Up to $5.6 \mathrm{~Hz}$ \\
\hline
\end{tabular}




\section{B.4 FlowMap Processor}

The FlowMap Processor contains a synchronization board, four input buffers with personality modules and four correlator units as shown in Fig. B.5. The Processor is connected to the gear pump system, the illumination system and the camera. This way, the TTL signal, the pulsing light-sheet and the recording of camera images can be synchronized through the synchronization board. The input buffer with personality module read image maps from the CCD camera, store images in memory, and feed them to the correlator for vector processing. All these events happen simultaneously, ensuring a continuous throughput of image maps from the camera and allowing realtime vector processing. In addition, an internal controller contained in the Processor is responsible for powering up the system and supplying a communication link via the Ethernet interface to an external PC on which the software package, FlowManager, is installed. The FlowManager provides the user interface to the system, control of the synchronizer, image capture, image analysis and post-processing of the PIV data.

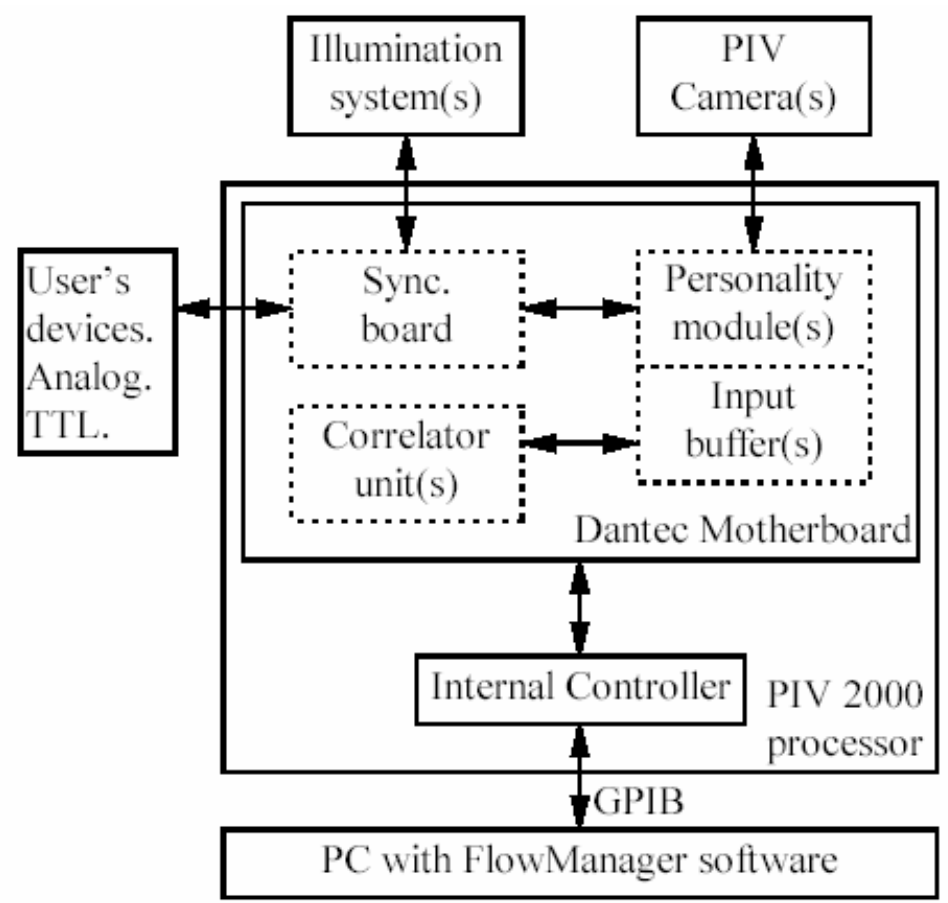

Fig. B.5 Architecture of the FlowMap PIV instrumentation. 